\title{
Internal representations of time and motion
}

\author{
Dissertation
}

zur Erlangung des mathematisch-naturwissenschaftlichen Doktorgrades "Doctor rerum naturalium " der Georg-August-Universität Göttingen

vorgelegt von

Joachim Haß

aus

Hofgeismar

Göttingen 2009 


\section{Mitglieder des Betreuungsausschusses:}

Prof. Dr. Theo Geisel (Referent)

Institut für Nichtlineare Dynamik, Universität Göttingen

Abteilung Nichtlineare Dynamik, MPI for Dynamik und Selbstorganisation

Bernstein Zentrum für Computational Neuroscience, Göttingen

Prof. Dr. Kristian Folta (Referent)

Forschungsgruppe Neurobiologische Grundlagen des Lernens,

Universität Hildesheim

Dr. J. Michael Herrmann

Institute of Perception, Action and Behaviour,

University of Edinburgh

Bernstein Zentrum für Computational Neuroscience, Göttingen

Prof. Dr. Stefan Treue

Abteilung Kognitive Neurowissenschaften,

Deutsches Primatenzentrum, Göttingen

Bernstein Zentrum für Computational Neuroscience, Göttingen

Tag der mündlichen Prüfung: 11. November 2009 
"Es gibt ein großes und doch ganz alltägliches Geheimnis. Alle Menschen haben daran teil, jeder kennt es, aber die wenigsten denken je darüber nach. Die meisten Leute nehmen es einfach so hin und wundern sich kein bisschen darüber. Dieses Geheimnis ist die Zeit 1․,

- Michael Ende, "Momo", 1973

\footnotetext{
1 "Life holds one great but quite commonplace mystery. Though shared by each of us and known to all, seldom rates a second thought. That mystery, which most of us take for granted and never think twice about, is time."
} 


\section{Statement of Originality}

I hereby declare that this thesis is my own work and has been written independently, with no other sources and aids than quoted in the text, references and acknowledgments.

Göttingen, September 2009

Joachim Haß 


\section{Abstract}

The way how humans perceive the passage of time is one of the greatest mysteries both in neuroscience and psychology. Compared to other perceptual quantities, relatively little is known about the neural basis and the psychophysical laws of time perception. In this thesis, we seek to contribute to the understanding of human time perception by combining knowledge from neuroscience and psychology into a neurocomputational modeling approach. The first two studies concentrate on timing errors, i.e. the variability in duration estimation, and its relation to physical duration, while the latter two are concerned with the issue of integrating temporal information from different sources into a unique representation of time.

Regarding timing errors, it is well known that the variability of durations estimates increases with the duration of the interval to be estimated. In the first study, we present a model based on neuronal networks called synfire chains which explains the functional form of the relation of these errors relative to interval duration. This form emerges from a constrained optimization involving a number of different chains, and can be implemented by means of a competitive plasticity rule applied to the synaptic connections from the chains onto a readout network. Previous models could only explain a linear increase of timing errors with duration (Weber's law), without providing a neural basis of this behavior. In the second study, we extend the modeling approach into a more general stochastic framework based on Fisher information. This approach follows from the idea that not only systematic changes in brain processes may be used as a source of temporal information, but also features of the stochastic "noise". We compute the scaling of the minimal timing errors on the interval duration, and found a hierarchy of temporal information, i.e. the scaling is best for estimates based on the systematic changes, and worst for correlations-based estimates. Specifically, we find that Weber's law can only be reproduced if variability is the only available source of temporal information.

In the third study, we investigate how the subjective duration of an auditorily presented interval is affected by motion that is either actively performed or visually observed. In a series of psychophysical experiments, human participants performed a rotor tracking task and an interval discrimination task with intervals of $100 \mathrm{~ms}$ at the same time. We find that subjective duration is affected by the speed, but not by the curvature of visually presented motion. Active performance of the motion does not contribute to this effect, but impairs discrimination performance acting as interference. The results show a direct cross-modal interaction between auditory and visual time perception, and a separation of the mechanisms from those underlying continuous motor timing. Attention is shown to modulate time perception even in the range of hundreds of milliseconds, but does not interact with the duration distortion by visual motion. Finally, the forth study is concerned with the integration of temporal information from multiple intervals presented within a sequence. We describe a model of an adaptive memory unit which is capable of computing an average over these presentations. The model explains the observation that an interval with a deviating duration can be more easily detected within a sequence of standard intervals if it is presented at a later position within the sequence.

Taken together, the results of these four studies suggest a framework of temporal processing where temporal representations of different durations and from different sensory modalities are integrated into a centralized "temporal hub", with relative weights determined by the reliability of their information (Bayesian integration), and are then processed by cognitive processes such as averaging in short-term memory. 


\section{Contents}

$\begin{array}{ll}\text { 1. Introduction } & 1\end{array}$

\begin{tabular}{ll}
\hline 2. Fundamentals & 7
\end{tabular}

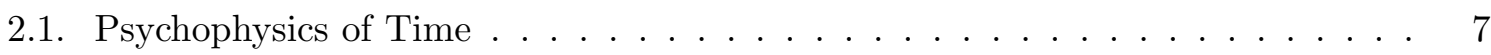

2.1.1. Objective measurement of subjective time perception . . . . . . . . . . 7

2.1.2. Relating subjective and phvsical time . . . . . . . . . . . . . 13

2.1.3. Information-processing models of time perception . . . . . . . . . . 20

2.2. (Computational) Neuroscience of Time . . . . . . . . . . . . . . . . . 27

2.2.1. Brain regions involved in temporal processing . . . . . . . . . . . . 28

2.2.2. Modelling local brain circuits . . . . . . . . . . . . . . . . 35

2.2.3. Computational models of time perception . . . . . . . . . . . . . 39

2.3. Information Theorv of Time . . . . . . . . . . . . . . . . 47

2.3.1. Stochastic inference . . . . . . . . . . . . . . . 47

2.3.2. Fisher information and time estimation . . . . . . . . . . . . . 49

2.3.3. Bavsian integration of temporal multi-sensory information . . . . . . . . 50

3. Time perception by optimal synaptic selection of synfire chains 53

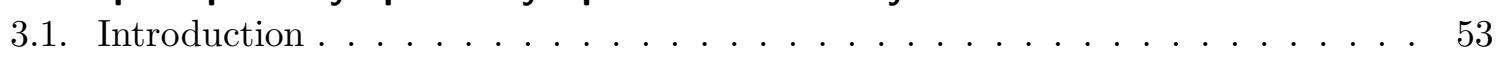

3.2. Neuron and network model . . . . . . . . . . . . . . . 56

3.2.1. Network structure . . . . . . . . . . . . . . . . . . . 56

3.2.2. Neuron model and synapses . . . . . . . . . . . . . . . . . . . . 58

3.2.3. Svnaptic plasticity . . . . . . . . . . . . . . . . . . 59

3.3. Temporal statistics of svnfire chains . . . . . . . . . . . . . . . 59

3.3.1. Quasi-spatial representation of time . . . . . . . . . . . . . 59

3.3.2. Timing errors . . . . . . . . . . . . . . . . . . 60

3.3.3. Parameter variations . . . . . . . . . . . . . . . . . . 62

3.4. Optimal temporal processing . . . . . . . . . . . . . . . . 65

3.5. Optimization by competitive STDP learning . . . . . . . . . . . . . . . . 67

3.5.1. Effective learning rate depending on timing errors . . . . . . . . . . . 68

3.5.2. Optimal selection of svnfire chains . . . . . . . . . . . . . . 69

3.5.3. Unique representation in readout neurons . . . . . . . . . . . . . . . 71

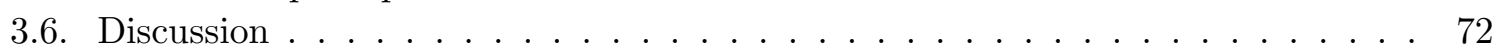

$\begin{array}{ll}\text { 4. Information-theoretical analysis of time perception } & 77\end{array}$

4.1. Introduction . . . . . . . . . . . . . . . . . . . 77

4.2. Estimation of temporal intervals . . . . . . . . . . . . . . . . . 79

4.3. Single Gaussian processes . . . . . . . . . . . . . . . . . . 80 
4.4. Multiple processes . . . . . . . . . . . . . . . . . . . . . . 82

4.4.1. The covariance-based model . . . . . . . . . . . . . . . . . . 82

4.4.2. The synfire chain model . . . . . . . . . . . . . . . . . 84

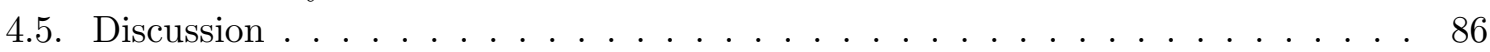

5. Cross-modal interaction of time perception and motor timing 89

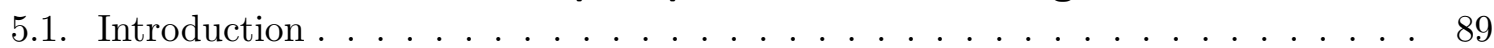

5.2. Results . . . . . . . . . . . . . . . . . . . . . . . 90

5.3. Discussion . . . . . . . . . . . . . . . . . . . 94

6. Cognitive aspects of time perception in temporal sequences 97

6.1. Introduction . . . . . . . . . . . . . . . . . . . 97

6.2. Sequence experiment . . . . . . . . . . . . . . . . . . . . . 98

6.2.1. Method . . . . . . . . . . . . . . . . . . . 98

6.2.2. Results . . . . . . . . . . . . . . . . . . . . 99

6.3. Serial memorv model . . . . . . . . . . . . . . . . . 100

6.3.1. Single interval representations by svnfire chains . . . . . . . . . . . . 100

6.3.2. Memory and decision stage . . . . . . . . . . . . . . . . . . . 102

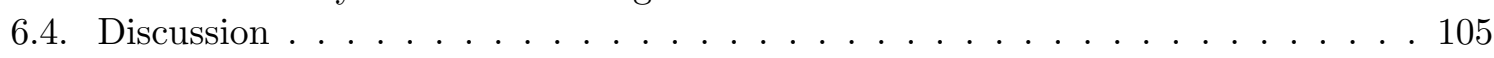

$\begin{array}{ll}\text { 7. Discussion } & 107\end{array}$

7.1. A general framework for temporal processing . . . . . . . . . . . . . . . . 107

7.2. Conclusion . . . . . . . . . . . . . . . . . . 112

\begin{tabular}{ll}
\hline Appendix & 117
\end{tabular}

$\begin{array}{ll}\text { A. Supplemental material for Chapter 5 } & 117\end{array}$

A.1. Materials and Methods . . . . . . . . . . . . . . . . . . . . . . 117

A.2. Supporting text . . . . . . . . . . . . . . . . . . . . 122

$\begin{array}{ll}\text { References } & 127\end{array}$

\begin{tabular}{ll}
\hline Acknowledgments & 138
\end{tabular} 


\section{Introduction}

When thinking about time, one observes that it seems to lead a strange dual life: On the one hand, time is so ubiquitous in our daily life that everybody has a clear intuition about what it is: Calendars and clocks of ever-increasing precision constantly remind us that time is flying by, and that everything we do takes a certain amount of this time. Thus, time is regarded as precious, and often, it is running out when we are late on an appointment or a deadline is approaching. Even in the language of all these examples, one sees how intimately time is inwoven in our lives. We have the feeling that time passes, and we are capable to interact with an outer world which is also changing in time. This is most obvious when our sense of time deviates from physical time, when time seems to fly by when we enjoy ourselves, or to stand still when we are anxious or bored. And we are also aware about an apparent directionality of time: It seems to flow from future to past, allowing for development and the evolution of events, but also leading to decay, aging, and ultimately death, the end of a limited life time.

On the other hand, beyond this intuitive and natural understanding, time poses itself as one of the most enigmatic phenomena of existence. The seemingly simple question "What is time?" has ignited fierce disputes among scientists and philosophers of all times [118. In Western history, this dispute begins which the ancient Greek philosophers Heraclitus and Parmenides, who formulated two fundamentally different views on time. For Heraclitus, the world is in a constant state of change, thus time and evolution are of the essence of the existence. Parmenides, on the other hand, perceived the world as static and considered time, motion and change as mere illusions. Both of these views on time remained influential in modern times. While Newton, Leibniz and many other philosophers considered time an aspect of actual reality, Kant regarded both space and time as "a priori" notions that allow us to comprehend reality, but not necessarily as aspects of reality itself. McTaggart [125 took an even more radical view and provided a strictly logical chain of arguments to show that time does not exist. Science also provides evidence for both views on time. The Parmenidian view of a static and constant world is reflected in the fact that the basic equations of motion in classical, relativistic, statistical and quantum mechanics are actually invariant to transformations of time. In Einstein's theory of special relativity, time even degenerates to one out of four dimensions of space-time. On the other hand, notions of change and evolution, which comply with the Heraclitian view on time, manifest themselves in irreversible processes, such as the global increase of entropy and the self-organization of complex systems such as life itself [32. To date, neither science nor philosophy has succeeded to resolve this and many other controversies about the nature of time.

The apparent discrepancy between the intuitive understanding of time and the continuing lack of a thorough understanding of its nature is concisely reflected upon in the words of the philosopher and church father Augustinus: "What then is time? If no one asks me, I know what it is. If I wish to explain it to him who asks, I do not know." 
A specific aspect of time is the question how temporal information is processed by the human observer. This topic is of particular interest, as it allows to investigate the basis of our everyday perception of time in a scientific way, and may thus act as a bridge between the intuition and the philosophy of time. The human brain has to deal with a wide variety of temporal information at time scales that reach from hundreds of microseconds to hours, days, and beyond: Our sense for the "flow of time" is based on the perception of the duration of events on all of these time scales. The planning and execution of any directed motor act requires precisely timed patterns of activation in large sets of muscles. The notion of the "present" results from our ability to decide whether two events happened simultaneously or in close succession [144]. The perception of complex temporal stimuli such as speech or music demands for discrimination of temporal sequences and rhythm perception. And as a final example, our sense of causality is based on correct judgments of temporal order.

Traditionally, investigations on all these abilities have been conducted by psychologists, who seek to relate subjective time and physical time by means of psychophysical laws. More recently, also neuroscientists gained interest into the brain mechanisms that enable the representation of time. However, many of the most fundamental questions of time perception are still open in both disciplines. From the literature (cf. Chapter 2), one can extract the following five basic questions which are recurring in the current debate:

1. Transfer function: How does subjective time relate to physical time? Subjective time is considered in a statistical sense here, based on the stochastic responses of a human to a temporal task. One asks what is the mean and the standard deviation of a human time estimate, as a function of the actual duration. While it is generally accepted that both the mean and the standard deviation increase monotonically with physical interval duration, the exact shape of these relations is debated (see Sections 2.1.2.1 and 2.1.2.2).

2. Non-temporal factors: How can non-temporal factors influence time perception? Subjective duration stretches and dilates in relation to many features of a stimulus or states of the brain that have nothing to do with physical time itself (see Section 2.1.2.3). Examples are the increased subjective duration of a moving stimulus compared to a static one, and the effect that diminished attention induced by a secondary task decreases subjective duration. The mechanisms of most of these duration distortions are not understood, although there are attempts to incorporate them into psychophysical theories. (Section 2.1.3.2).

3. Neural code: Which temporal properties of neural networks in the brain are used to encode time? Any brain process that evolves in time would be a feasible candidate for this code, given that its temporal information is accessible. Accordingly, many neurocomputational models of time perception have been proposed, but it is not clear whether any of them is a valid model of time perception of the brain, or whether they are mutually exclusive. (see Section 2.2.3).

4. Localization: Which brain structures are involved in the representation of time? The rich repertoire of neuroscience methods has revealed a wide range of brain regions that are likely to be involved in time perception. However, none of them could be proven to be critical for temporal processing, and the respective function of each region is strongly debated. (see Section 2.2.1). 
5. Unity vs. Diversity: Is there a common representation of time for a) different sensory modalities, b) time perception and motor timing and c) second and millisecond intervals? And if there are different representations for any of these dimensions, in what respects do they differ from each other, and are they completely disjoint, or are there interactions? There is some evidence that different mechanisms exist for time perception in the range of milliseconds and seconds (see Sections 2.1.2.3, 2.2.1 and 2.2.3), and that continuous motor timing is disjoint from both time perception and discrete motor timing (Section 2.1.1.1), but the evidence for both proposals is ambiguous. The same is true for time perception in different modalities, where both evidence for a centralized "internal clock" and for modality-specific timing mechanisms was provided (see Section 2.1.2.3 and 2.1.3.2).

While question 1 and 2 about the transfer function and the role of non-temporal factors were traditionally pursued by psychophysical researchers, question 3 and 4 about the neural localizations and mechanisms originate from neuroscience research. These separate sets of questions are also reflected in the theoretical models that are proposed by the two disciplines: Psychophysical theories are rarely grounded on neural mechanisms, and most neurocomputational models do not make predictions about psychophysical experiments. This separation has only recently begun to be overcome. Question 5 of whether time is represented by a single or multiple mechanism is one of the examples where both research communities investigate the same questions with different means. At any rate, the above list demonstrates how little definite knowledge exists in the field of time perception, compared to other fields of neuroscience and psychology. "The field of temporal processing is still at its infancy.", stated Dean Buonomano in his 2002 review on the perception of time [28]. The enigma of the nature of time seems to reflect itself in the enigmas of its perception.

In this work, we attempt to contribute to answer the five questions stated above by means of an integrative investigation that links psychophysical and neuroscientific approaches in the framework of computational neuroscience and information theory. We focus on mechanisms for the representation of interval duration in the range of hundreds of milliseconds, although many of our results are also applicable to intervals in the range of seconds to minutes. For much longer and much shorter intervals, in the range of a day or several hundred microseconds, temporal information is represented by fundamentally different mechanism compared to the intermediate range we investigate [28, 24], and we do not consider these extreme cases. Likewise, we do not explicitly consider order judgment or the discrimination between simultaneous and successive events. However, we do show how the representation of sequences may emerge from single-interval representations. In general, our work is guided by the assumption that the brain follows the principle of constrained optimality: Given its resources and limitations, the brain attempts to extract as much information as possible out of a given stimulus. If this principle holds for time perception, it should be possible to see traces of optimality in the psychophysical responses, and to identify constraints within the details of the neural implementation.

The thesis is organized as follows: After a review of the literature and the introduction of important concepts of psychophysics, neurocomputational modeling, and information theory 
in Chapter 2, Chapter 3 to 6 present our original work on different aspects of temporal processes. Chapter 3 and 6 contains material that has been previously published in slightly modified form. The thesis is concluded by a discussion in Chapter 7 which shows how the results from all of the chapters converge into a unified framework.

Chapter 3 and 4 of the thesis are concerned with the representation of duration of intervals that are presented within a single modality. In order to constrain our choice of neurobiological models, we demand that it must be able to reproduce psychophysical observations about the errors of duration estimate in humans. In this way, we combine question 1 about the psychophysical transfer function with question 3 about the neural code. In accordance with neurobiological findings, we assume that single-interval durations are represented by cortical networks. In Chapter 3, we consider a specific computational model of cortical function, the synfire chain. The model consists of a number of pools of neurons with feed-forward connections. It has been shown that neural activity may stably propagate through a synfire chain with a reliable transmission speed and small temporal jitter in each pool. These properties make the synfire chain a well-suited candidate for the neural representation of time by means of a quasi-spatial code. Using this model, we aim to reproduce the experimentally observed dependency of timing errors on duration. Current experiments suggest that the standard deviation of a time estimate increase linearly with duration at intermediate interval length (a dependency called Weber's law), sub-linearly for shorter intervals, and super-linearly for longer intervals. This form of the error function is not easy to be modeled, as the accumulation of independent noise in neural system only predicts timing errors that increase with the square root of duration. We show that the experimentally observed error function can be obtained as a result of constrained optimization: If the chain is considered to be of a finite length, longer intervals can only be represented when a chain with a slower transmission is used. This leads to the assumption of multiple synfire chains with different transmission speeds: We show that slower propagation of synfire activity leads to a superlinear increase in the variability in the propagation, no matter which model parameter is used to modify the propagation speed. In this way, the need for slower propagation introduces an additional error source, which leads to the experimentally observed profile of timing errors. Finally, we show how the selection of the optimal chain for each time interval could be achieved when the individual synfire chains project to a centralized read-out network. A plasticity rule combining spike-timing dependent plasticity (STDP) and homeostatic plasticity enables the detection of differences in the variability of the different chains, and a synaptic competition that is won by the optimal chain for each duration.

In Chapter 4, we generalize the idea of predicting the psychophysical error function from neurobiological models and introduce a statistical framework that enables to compare the ability of arbitrary models to make such predictions. The framework is based on information theory and considers any brain process that attempts to extract temporal information from a noisy environment as an inherently stochastic process, and a psychophysical judgment about time as an estimation problem. Within this framework, it is possible to compute the lowest possible timing error for a given stochastic process, which is the Cramer-Rao bound, the inverse of the Fisher information. In this sense, an estimate can be considered optimal when its variance reaches this lower bound. Under quite general assumptions, we find a hierarchy of temporal information in the mean, the variance and the covariance of such a process: The lower bound for the timing errors scales with the square root of the duration of the estimated 
interval if only the information from the mean is used, but is linear in the duration when relying on the variance alone, and even increases exponentially with the interval duration when using only the covariance. Furthermore, we extend this framework to the case where information from multiple processes is available at the same time. By interpreting of the synfire model from Chapter 3 as set of multiple stochastic processes, we show that the optimal time estimate we obtained is also the optimal solution in the mathematical sense. We also consider another model which represents time solely by exponentially decaying covariance. Contrary to the claim of the authors [5], we show that the timing errors in this model increase exponentially with the interval length .

In Chapter 5, we turn to question 5 and study the interaction of different modes of temporal processing, namely time perception in different sensory modalities and continuous motor timing. Different from previous research, which mainly used correlational evidence to link these different modes, we employ a distortion approach, exploiting the fact that time perception can be manipulated by non-temporal factors (question 2). More specifically, we conducted a series of psychophysical experiments comprising a time perception task and a simultaneously performed motor task to study the effects of visually observed and actually performed motion on auditory time perception. Participants were required to perform an arm movement following an elliptic trajectory that was prescribed by a moving target on a screen. At specific segments of the motion, namely the apices of the ellipse, they were presented with two successive auditory stimuli, which they had to discriminate according to their duration. We find that the observation of the motion of the visual target distorted subjective duration, such that duration was perceived as longer when the observed motion was faster. Such a distortion is well described for visual intervals, but our study is the first to show that this effect carries over to the auditory domain. On the other hand, control experiments showed that the actual performance of the motion does not contribute to this effect, suggesting a separation of the mechanisms for time perception and continuous motor timing. Furthermore, we show that the distortion effect does not depend on a global change of attention. However, the concurrent performance of a secondary motor task together with the time perception task is shown to decrease the performance to discriminate the two intervals. Such dual-task interference has mostly been studies for interval durations in the seconds range, but our results support the notion that this distortion is also possible in the milliseconds range. Overall, the results are best explained by Bayesian integration of temporal information from the different sensory modalities into a centralized "temporal hub", which may be subject of attentional modulation, but is independent from the mechanisms that govern the timing of continuous motion. Again, this form of integration is the optimal solution given the problem to extract temporal information from independent sources with different variability.

Finally, Chapter 6 extends the scope of our work from single intervals to sequences of intervals. Experiments that were conducted by our collaborator Stefan Blaschke showed that the ability to detect a deviant interval in an isochronous sequence of identical standard intervals depends on the position of the deviant within the sequence: The more standard intervals are presented prior to the deviant, the easier is the detection task. This observation suggests that the brain uses the information that is contained in a sequence to build a more robust overall representation of the standard duration of the single intervals. We present a simple formal model which explains this phenomenon as a result of an averaging process over the intervals presented in the sequence. This averaging takes place within a serial memory stack system 
with two components, one that provides a short-term storage of a single interval, and a second one which integrates over all the intervals that were previously presented. We present a formal derivation of how this architecture lead to increasing detection performance at later positions of the deviant, and discuss predictions of the model for further sequence experiments.

The combination of our results with previous neuroanatomical and physiological evidence suggests a specific flow of temporal information through a network of brain regions (question 4): Sensory stimuli are primarily processed in the cortical areas that correspond to their respective modality, such as the primary visual or auditory cortex. As a by-product of these principle computations, the temporal information in the stimulus is also extracted (Chapter 3). Whenever the task at hand contains a temporal component, the temporal representations from all available cortical processes are projected onto a central brain site, which takes the function of weighting all incoming input by their respective reliability (Chapter 3 and 5 ). Following the converging evidence from neuroscience studies, this "temporal hub" is likely to be located in the striatum (cf. Section 2.2.1). When an integrated representation of an interval duration has been formed in this way, it is projected back into the neocortex, where it is stored in working memory circuits in the prefrontal cortex, and may be used to make temporal decisions or be subject of further cognitive manipulations (Chapter 6). In the discussion in Chapter 7 , we consider this framework in more detail, discuss predictions and limitations, and suggest further experiments which can be used to test the validity of our propositions. 


\section{Fundamentals}

\subsection{Psychophysics of Time}

Studies of temporal processing have a long tradition in psychophysics and were already conducted by one of the founders of this discipline, Fechner [47], and his contemporaries Höring [74], Mach [115] and Vierordt [177]. The general aim of psychophysics is to study the relation between the physical properties of a stimulus, such as duration, and measurable correlates of the subjective impression of that property, such as subjective duration. Usually, the subjective quantity will not solely depend on the physical stimulus property, but may be influenced by various other factors. As an example from auditory perception, the impression of the loudness of a tone does not only depend on the sound pressure the ear is exposed to, but also on the frequency at which the tone is presented [100]. Such non-intuitive dependencies allow insight into the mechanisms of perception, without using the more sophisticated neuroscience methods that are frequently used today. The advantage of psychophysics over these methods is that the experiments are relatively simple and inexpensive and do not require specific knowledge about the location and nature of brain circuits involved in the task to be studied. This method thus constitutes an ideal starting point for a field like temporal processing where these details are largely unknown (cf. Section 2.2).

\subsubsection{Objective measurement of subjective time perception}

\subsubsection{Experimental paradigms}

To objectively assess the perception of time in humans, psychologists ask participants to perform behavioral tasks in which the dimension of time plays a critical role. These experiments allow the calculation of estimators of temporal performance (dependent variables), and to relate them to parameters that can be experimentally manipulated (independent variables). Here, we describe a number of experimental paradigms that are used to assess the estimation of the duration of an interval, and explain how one can derive two estimators that are of particular relevance to our studies: The mean and the standard deviation of a duration estimate.

Important classes of experiments include interval discrimination, interval reproduction, finger tapping and arm movement tasks, although this list is by no means complet 1 . In interval discrimination, participants are presented with two stimuli of similar duration and their task

\footnotetext{
${ }^{1}$ More comprehensive overviews over the various types of experiments for studying temporal processing can be found in [7, 186]. (time perception in humans), [108] (time perception in animals) and 190] (human experiments related to motor timing).
} 


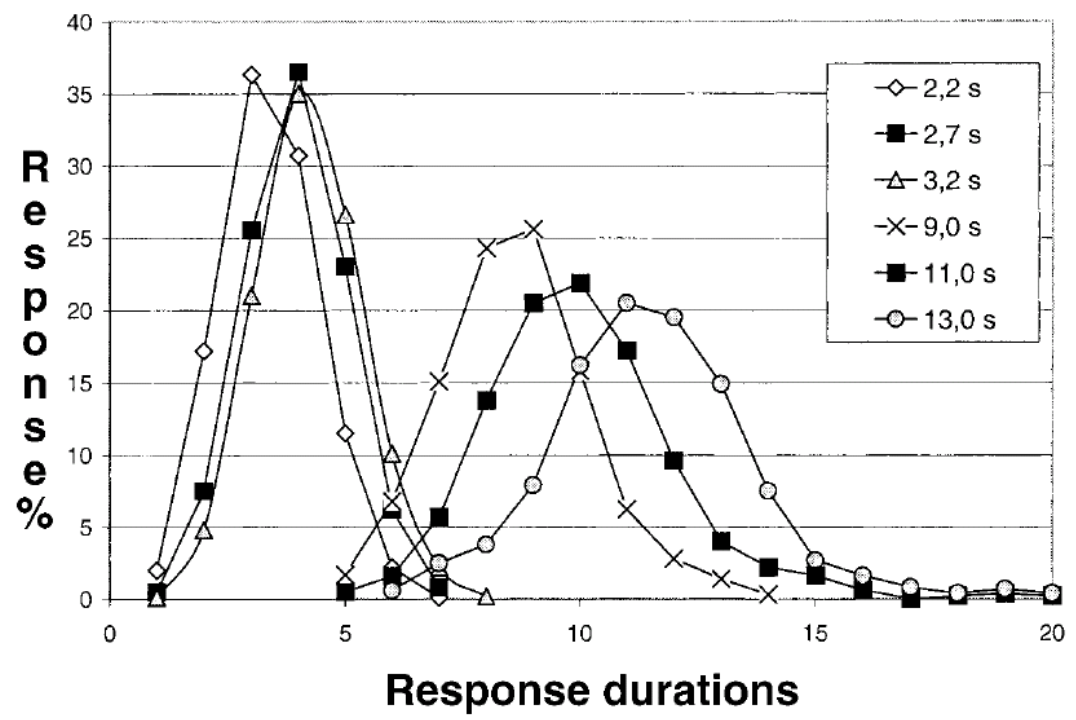

Figure 2.1.: Response rates in a interval reproduction task for different target times given in the legend [112. The distributions can be well fitted by Gaussians with different means and variances for all interval durations. With kind permission from Springer Science+Business Media: Experimental Brain Research, "Activation of the supplementary motor area and of attentional networks during temporal processing", 142 (2002) 475-485, F. Macar et al., figure 1.

is to judge which of them appeared longer to them. One of those stimuli is usually of constant duration (standard), while the other is systematically changed (comparison). From the fraction of false and correct judgments for different durations of the comparison, one can extract the just noticeable difference (jnd) between the two stimuli, which is also referred to as difference limen (DL), and the point of subjective equality (PSE), the duration at which the comparison appears to be as long as the standard (details of this procedure are given below).

The point of subjective equality and the difference limen are of central interest for the psychophysics of time because they can be seen as the mean (PSE) and the standard deviation (DL) of an estimate of interval duration in the brain. PSEs close to the actual standard duration indicate a high accuracy of the estimate, i.e. a small estimation bias. Distortions of subjective time lead to an over- or underestimation of duration. In interval discrimination, an overestimation of the comparison compared to the standard is reflected in a PSE below the standard, as the overestimated comparison must be shortened to be perceived as equal to the standard. In the same way, underestimation of the comparison leads to a PSE abve the standard. The DL reflects the temporal jitter in the internal representations of time and constitutes a measure of the reliability of the time estimate, which is reflected in the just noticeable difference in interval discrimination. However, the DL must be interpreted with care, as there may be different sources of variability, some of which being related to nontemporal factors such as response variability or memory limitations. We will elaborate on this issue in Section 2.1.3, 
Measures that are equivalent to PSE and DL can be obtained in the paradigm of interval reproduction. Participants are presented with a standard interval and have to reproduce this interval afterwards, e.g. by pressing a button as long as they think the standard interval was. The result is a histogram of reproduced intervals, which can usually be fitted by a Gaussian distribution (Fig. 2.1). The mean and the standard deviation of this distribution correspond to the PSE and the DL, respectively.

In finger tapping experiments, a rhythm is presented, usually by auditory beats similar to a metronome, which participants have to reproduce by tapping with a finger onto a plate where each tap is recorded. The mean and the standard deviation of the interval between successive taps (inter-tap interval) can then be interpreted similarly as the PSE and DL, respectively. Furthermore, as this experiment is based on a rhythms rather than single intervals, one can apply higher-order measures such as the spectrum of tapping frequencies and correlations between successive inter-tap intervals [190].

Finally, arm movement tasks comprise experiments in which participants have to follow a target which their hand (tracking tasks) or to reach for a target at a distance (reaching task). In the former class of experiments, the time lag between target and actual motion is used as a measure of temporal performance, whereas for latter one, the time in which the target is reached can be used. The mean and standard deviation of these measures can be seen as analogons to PSE and DL, respectively.

The precise relation between time perception and motor timing tasks is currently debated. Studies that link those two domains show that measures of performance are correlated for time perception and motor timing tasks [93, 151, 78, and that training in the perceptual domain entails increased performance in motor timing [132]. On the other hand, other studies have reported differences between time perception and motor control by showing that reaching relies on state-dependent rather than temporal representations [91, 35]. Recently, the notion of "emergent" vs. "event" timing has been introduced as an attempt to resolve this apparent contradiction [190]. According to this hypothesis, continuous motor timing does not rely on explicit representations of time, but emerges from self-organizing control of non-temporal parameters of motion, such as velocity [103]. Time perception and discrete motion that relies on a temporal event structure, on the other hand, requires an explicit timekeeper which may be the same for the perceptual and the motor domain. Support of this hypothesis has been drawn from correlations in performance that are present between time perception and tapping [189], but neither between continuous circle drawing and tapping [155, 189] nor circle drawing and time perception [189]. However, results from pure correlation studies are not sufficient to confirm or deny a common mechanism for two modes of temporal processing, as it is always possible that an observed correlation in performance is due to commonly used resources beyond the actual timing mechanism, or that an existing correlation is not observed because of a large variability in the data (c.f. [28]). In Chapter [5, we provide causal support for the "emergent" vs. "event" timing hypothesis.

\subsubsection{Measuring subjective duration and discrimination performance}

In this work, we focus on the PSE and the DL as dependent variables, and employ the interval discrimination task to measure them. While any of the other methods could be 
used as well, we chose this one because it minimizes the role of motor responses. This is of particular importance for the experiments described in Chapter 5 where we assess possible relations between time perception and motor timing. A strong motor component in the time perception task could constitute a confoundation for this relation. Furthermore, interval discrimination is believed to be more suited for studying time perception in the range of hundreds of milliseconds compared to interval reproduction, because the production of such a short interval is a demanding task and may introduce additional error sources [151].

In the following, we describe how one can extract the PSE and the DL from an interval discrimination task. The two major procedures available are the method of constant stimuli and the weighted up-down method. The method of constant stimuli attempts to estimate the complete psychometric function of a duration estimate, i.e. the probability of a given response as a function of the duration of the comparison interval. If the participant responds according to both his perception and the instructions he was given, the probability of responding "interval two was longer" approaches one if the second interval is actually much longer than the first one. Conversely, this probability will approach zero if the second interval is much shorter than the first. For durations between these extremes, the probability of this particular response will gradually change from zero to. This shape of the psychometric function corresponds to the class of sigmoid functions, and it can often be well described by a cumulative Gaussian distribution, confirming the implicit assumption that the distribution of time estimates is Gaussian (cf. Fig. 2.2) [53].

The method of constant stimuli [46, 53] attempts to reconstruct this psychometric function by using a number of different (constant) durations for the comparison interval, and estimates the probability of the above response by presenting comparisons a number of times for each duration and calculating the frequency of this response. The result of a typical experiment is shown in Fig. 2.2, upper panel. Based on these probability estimates, a cumulative Gaussian distribution can be fitted the data (Fig. 2.2, lower panel). The DL and PSE can be directly calculated from this fit. By definition, the point of subjective equality is the comparison duration at which standard and comparison are perceived as being of equal duration. In the fitted curve, this is the point where the probability is equal to $50 \%$ (Fig. 2.2. lower panel). Similarly, the DL can be calculated from the inclination of the psychometric function around the PSE. As the function is non-linear, this inclination is approximated by using two points which lie symmetrically around the PSE (usually the 25 th and 75 th percentile $T_{25}$ and $T_{75}$ 2 ) and the formula

$$
D L \propto \frac{T_{75}-T_{25}}{2} .
$$

These percentiles also offer an alternative way of calculating the PSE by the formula

$$
P S E \propto \frac{T_{75}+T_{25}}{2} .
$$

\footnotetext{
${ }^{2}$ The choice of $T_{25}$ and $T_{75}$ is a historical one and makes the DL equivalent to the so-called semi-interquantile range. When choosing $T_{84}$ and $T_{16}$ instead, the DL equals the standard deviation, cf. [15.
} 

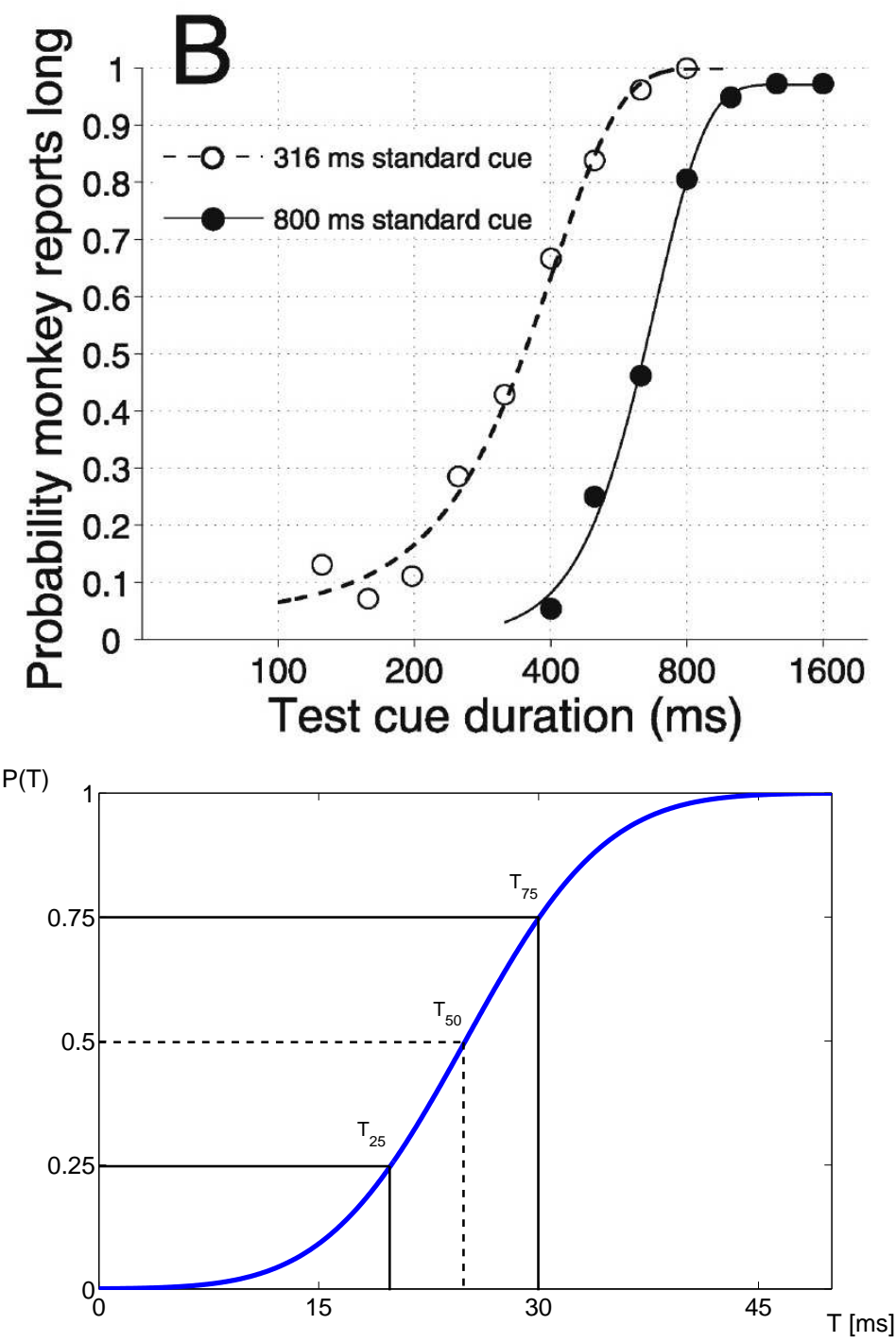

Figure 2.2.: Upper panel: Psychometric function in a typical interval discrimination task performed by a macaque monkey for two different base durations [109]. Dots show the frequency at which the monkey judges a visual interval as longer as the memorized base duration. Lines represent the cumulative Gaussian distributions to the data. Lower panel: Model the the psychometric function by a cumulative Gaussian distribution with mean $25 \mathrm{~ms}$ and variance $7.5 \mathrm{~ms}$. $T_{25}, T_{50}$ and $T_{75}$ denote the times at which the psychometric function has its 25 th, 50th and 75th percentile, respectively. Reprinted from Neuron, 38, M. I. Leon and M.N. Shadlen, "Representation of time by neurons in the posterior parietal cortex of the macaque", 317-327., Copyright (2003), with permission from Elsevier. 


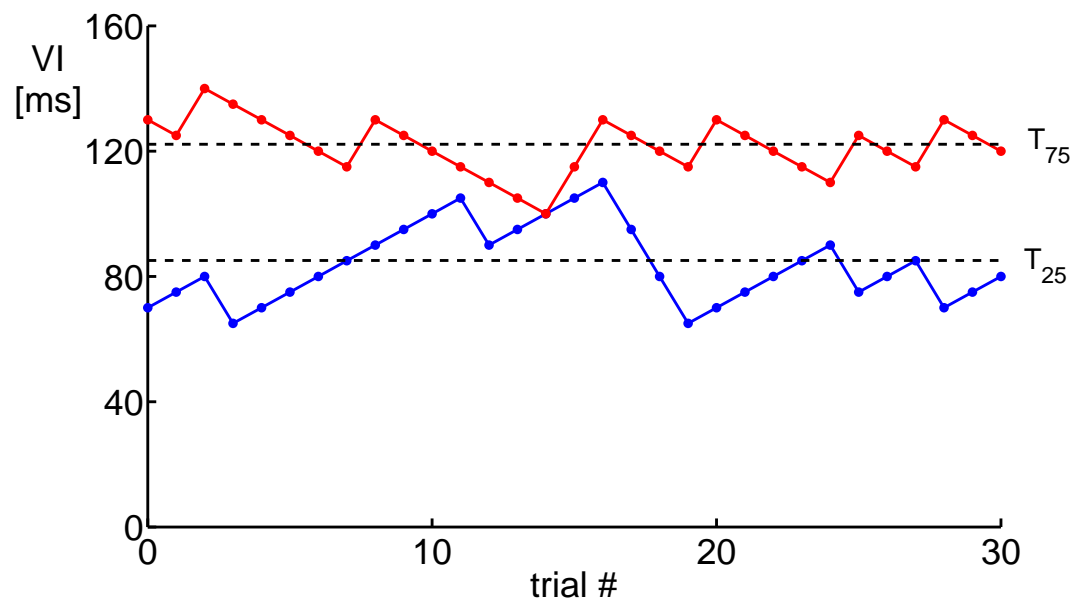

Figure 2.3.: Evolution of $T_{75}$ (red curve) and $T_{25}$ (blue curve) in an adaptive procedure [86]. The data is from our own experiment we describe in Chapter 5 . The average over the two runs starting from 130 and $70 \mathrm{~ms}$ (dotted lines) can be used as estimates of percentiles $T_{75}$ and $T_{25}$ of the psychometric function, respectively (cf. Fig. 2.2). See text for details.

A more direct way to access PSE and DL is given by adaptive procedures [106] such as the weighted up-down method [86. Here, no attempt is made to approximate the entire psychometric function, but the comparison duration is adapted such that it converges to two defined percentiles of the above response, from which both DL and PSE can be computed according to Eq. 2.1 and 2.2. This is accomplished by presenting the stimulus-comparison pair in two independent runs. In one run, the initial comparison duration is well above the standard duration, in the other, it is well below. In both runs, the duration of the comparison is changed such that it is closer to the standard ("step-down") if the participant makes a correct response, such that the discrimination task becomes harder. Conversely, the distance between comparison and standard is increased ("step-up") if the participant elicits a wrong response, which makes the task easier. By choosing the ratio of the step-up and step-down sizes appropriately, this procedure makes the comparison converge to any prescribed percentile of the response "tone two was longer". To see how this works, consider we want the comparison to converge to the $75 \%$ percentile of the above answer, given that we chose a standard of $100 \mathrm{~ms}$ and a comparison well above this value. If the comparison duration is precisely at the $75 \%$ percentile, the probability of the correct judgment that "tone two was longer" is three to one, by definition of the percentile. Thus, to keep the comparison duration at that value, the step-up value that follows a wrong response must be three times higher than the step-down value following a correct response. At this ratio, the changes add up to zero in the mean, and the comparison duration stays constant. By the same logic, if the comparison duration is at a value higher than the $75 \%$ percentile, using the three to one ratio of step-up 
and step-down sizes will decrease the comparison duration on average, and a value below the $75 \%$ percentile will increase. Thus, the comparison duration either oscillates around the true $75 \%$ percentile, or it converges to it. Other percentiles such as the $25 \%$ one can be reached by changing the step size ratio accordingly. Fig. 2.3 shows a typical evolution of the comparison durations for two runs starting at $130 \mathrm{~ms}$ and $70 \mathrm{~ms}$, respectively, and a standard of $100 \mathrm{~ms}$. The percentiles can be computed by averaging the comparison durations across trials, while discarding a number of initial values that reflect the transient phase.

Adaptive procedures like the weighted up-down method offer several advantages over the method of constant stimuli [106]. Given appropriate step sizes, the comparison durations converge relatively fast to the desired percentiles, such that they can be computed within less trials then would be needed for a reliable estimate of the psychometric function. Furthermore, the method can be beneficial for the motivation of the participants. During an experiment using the method of constant stimuli, participants might spend a lot of time in a regime where the task is too easy or too hard for them. This possibility is excluded by the fact that task difficulty is adapted to the participant's performance. For the same reason, the method is suitable even if the psychometric function differs a lot across participants, without major adjustments of parameters. An obvious disadvantage of the method is the fact that the information about the full psychometric function is discarded, so no statements beyond the PSE and the DL can be made. Furthermore, some studies suggest that the weighted up-down method does not produce exactly the same results than the method of constant stimuli [106]. However, evidence for this is ambiguous, and results from a recent study [104] suggest that adaptive procedures and the method of constant stimuli produce identical results for both DL and PSE. In any case, this would only be an issue when comparing absolute values of the results of studies using different methods.

\subsubsection{Relating subjective and physical time}

\subsubsection{The psychophysical law}

The psychophysical law describes how the subjective magnitude of a stimulus features changes as a function of the physical magnitude of that feature. The general form of this relation is nowadays accepted to be described by a power law 163

$$
E=k R^{\alpha}+b,
$$

which is also known as Steven's law. The exponents vary a lot between different stimulus features. Stevens reported $\alpha=0.67$ for loudness, $\alpha=0.33$ for brightness and a value as high as $\alpha=3.5$ for electric shock [163]. For time perception, Stevens reported $\alpha$ to be 1.1 [163]. Eisler, however, performed an extensive review of over hundred studies, spanning the range of $3 \mathrm{~ms}$ to $300 \mathrm{sec}$, and reported that the mean exponent was about 0.9 [43]. The fact that exponents do not deviate much from unity in many cases has lead researchers to believe that the relation between subjective time and physical time is linear

$$
E=k R+b .
$$

For instance, Allan [7] argued that that a linear function (Eq. 2.4) would actually fit some of the data better than a power function, and that some of the studies in favour of a power 


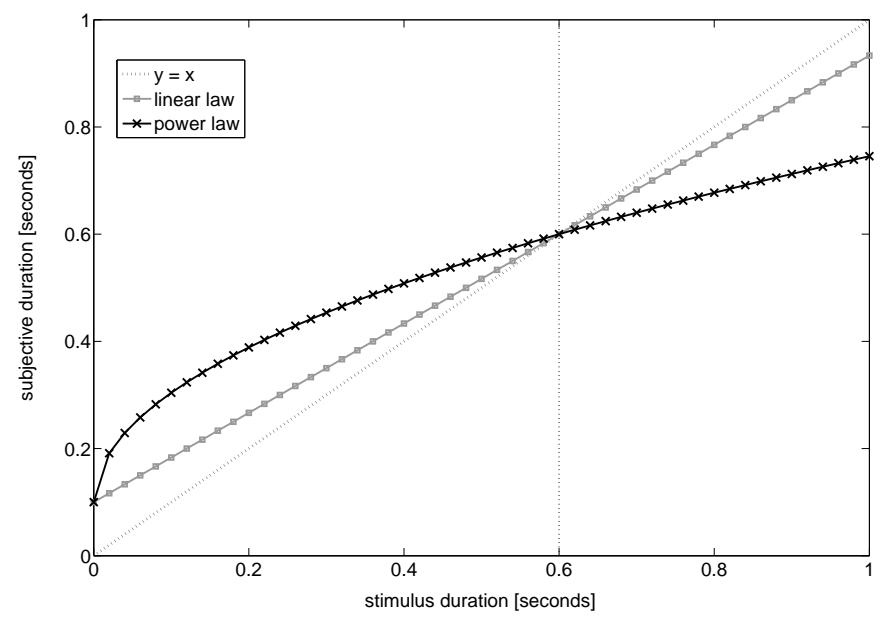

Figure 2.4.: Models of the psychophysical law by a linear and a power law function, together with the identity function [122. Both models incorporate Vierordt's law [177, which states that short intervals are overestimated, but longer intervals are underestimated. An indifference interval at $600 \mathrm{~ms}$ is also reproduced by both models.

function with $\alpha \neq 1.0$ may have used questionable methodology. This view is supported by Fraisse [50] and more recently by Lejeune and Wearden, who reviewed a number of both animal 108 and human [186 time perception studies. However, there are several cases where exponents deviate substantially from unity ( $\alpha$ ranged from 0.53 to 1.36 in [43], even if several problematic cases are excluded), so this issue is still debated.

Regarding the steepness $k$ of the function, there is a frequent observation, predominantly in interval reproduction tasks, which some researchers call Vierordt's law (after its discoverer Karl Vierordt [177, a pioneer in the psychophysics of time perception): Duration is perceived as longer than physical duration when intervals are short, but underestimated when intervals are long (Fig. 2.4) [186, 50, 7, 49]. The notion of long and short intervals is highly ambiguous here, as the so-called indifference interval at which subjective duration and physical duration coincide (Fig. 2.4) varies as lot between individuals and experimental conditions. However, the average value is close to $600 \mathrm{~ms}$ [49, 74]. Vierordt's law supports the notion that the steepness $k$ should be smaller than one and the offset $b$ should be larger than zero. However, the law can not be used to distinguish between linear and power functions for the psychophysical law, as both can be used to fit the data with the same indifference interval (Fig. 2.4).

\subsubsection{Weber's law for temporal variability, and its deviations}

Apart from the functional relation between the mean of the temporal estimates and the objective duration, the change of temporal variability with duration has also been extensively studied. This variability is consistently found to monotonically increase with the duration 

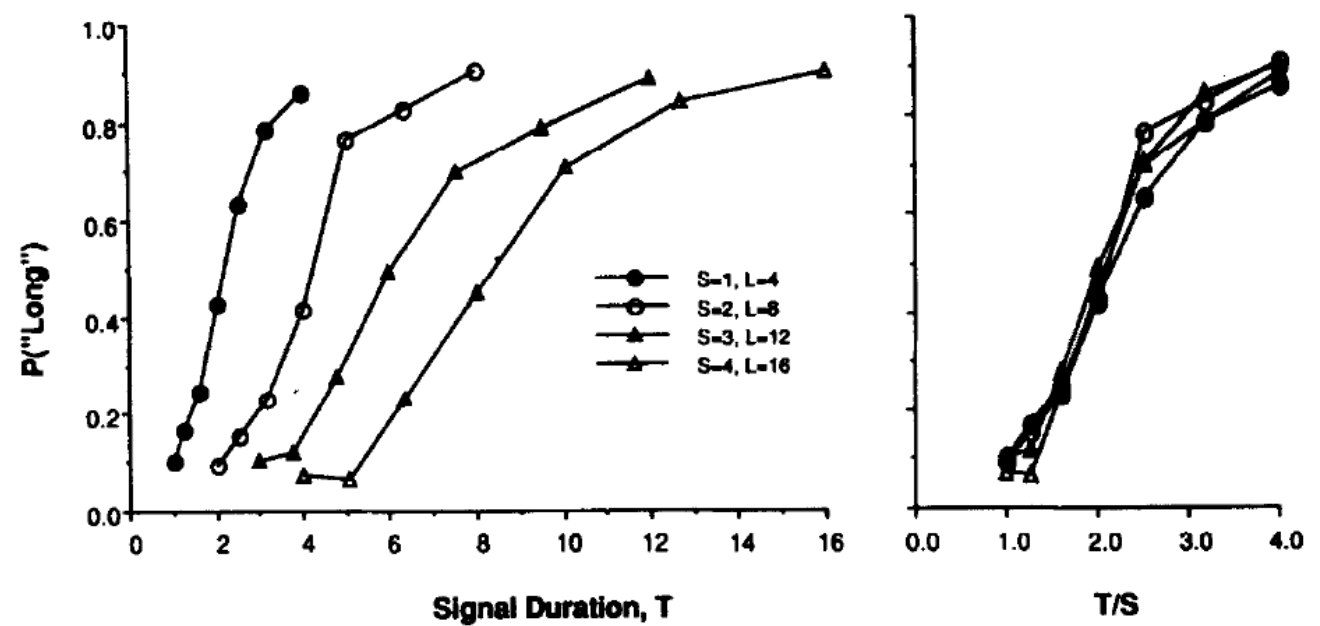

Figure 2.5.: Illustration of the scalar property of time perception [56]. Left panel: Psychometric functions in a bisection task performed by rats. The animals had to categorize intervals as "short" or "long" according to two memorized reference intervals $S$ and $L$. Curves show the probability that subjects judge an interval of duration $T$ as "long" as a function of $T$ for different values of $S$ and $L$. Right panel: When $T$ is scaled with the duration of the short interval $S$, the psychometric functions collapse onto a single one, showing that the entire psychometric functions scales with $S$. Reprinted from Learning and Motivation, 22, J. Gibbon, "Origins of scalar timing", 3-38., Copyright (1991), with permission from Elsevier.

of the physical interval increases. However, experimental evidence for the exact functional relation between duration and measures of temporal variability is much more ambiguous. A prominent proposal is that time perception follows Weber's law [46]. In its original form, this laws states that the just noticeable difference $\Delta R$ between two nearby values of the physical quantity increases linearly with the absolute value of that quantity $R$, resulting in a constant ratio

$$
W=\frac{\Delta R}{R}=\text { const },
$$

where $W$ is called the Weber fraction. This law implies that the variability of the estimate of the physical quantity increases linearly with this quantity. A related way of stating Weber's law is to demand that the coefficient of variation (cov) it constant, which relates standard deviation $(\sigma)$ to the mean $(\mu)$ of the estimate of $R$, rather than to $R$ itself

$$
\operatorname{cov}(R)=\frac{\sigma(R)}{\mu(R)} .
$$

Finally, a stronger version of Weber's law states that the entire probability distribution function (PDF) scales with the intensity of the physical stimulus dimension. That is, one can superimpose the PDFs if one scales the corresponding $\mathrm{x}$-axis such that all PDFs are centred 


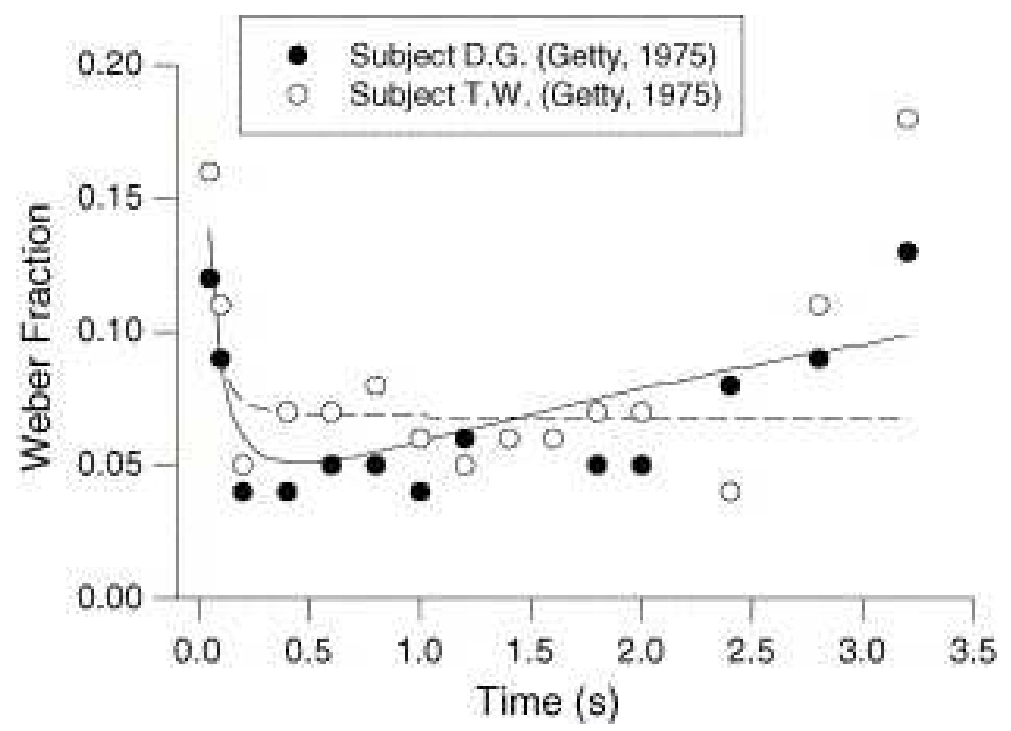

Figure 2.6.: Weber fraction as a function of the interval duration from a classical interval discrimination study by Getty [54, reprinted by Bizo and coworkers [15]. Apart from the data from two participants, two fits of the underlying Weber function are shown, one to the generalized Weber law (dotted line, cf. Eq. 2.7), and another one to a polynomial of the order $3 / 2$. The nonlinear function provides a better account for the data, as it captures the increasing Weber fraction at longer intervals. Reprinted from Behavioural Processes, 71, L. A. Bizo et al., "The failure of webers law in time perception and production", 201-210., Copyright (2006), with permission from Elsevier.

at the same mean (Fig. 2.5). This "scalar property" [55] is reflected in psychometric functions and other derived distributions that scale in the same way.

It has been shown that Weber's law holds for many perceptual dimensions, such as brightness, loudness, pitch, weight and temperature over a varying range of the corresponding physical intensities [46], and it has been a major guidance for psychophysics since its origins. However, many of the dimensions where Weber's laws holds are based on stimulus energy, such as the intensity of light or sound of the mass of an object. For such energy-based stimulus features, Weber's law is often caused by low-level adaptation. For instance, the fact that the just noticeable difference in light intensity increases approximately linear with the intensity over three orders of magnitude can be explained with an adaptation as early as in the retinal cone cells [139. Such a simple explanation is not available for time perception, as time is no energy-based quantity.

Still, a number of studies report that Weber's law is fulfilled in time perception 3 . This result was originally most frequently reported in animal studies [55, 56, 108, but numerous

\footnotetext{
${ }^{3}$ For brevity, we restrict ourselves to review articles and representative original articles, as there is a vast literature on the topic.
} 


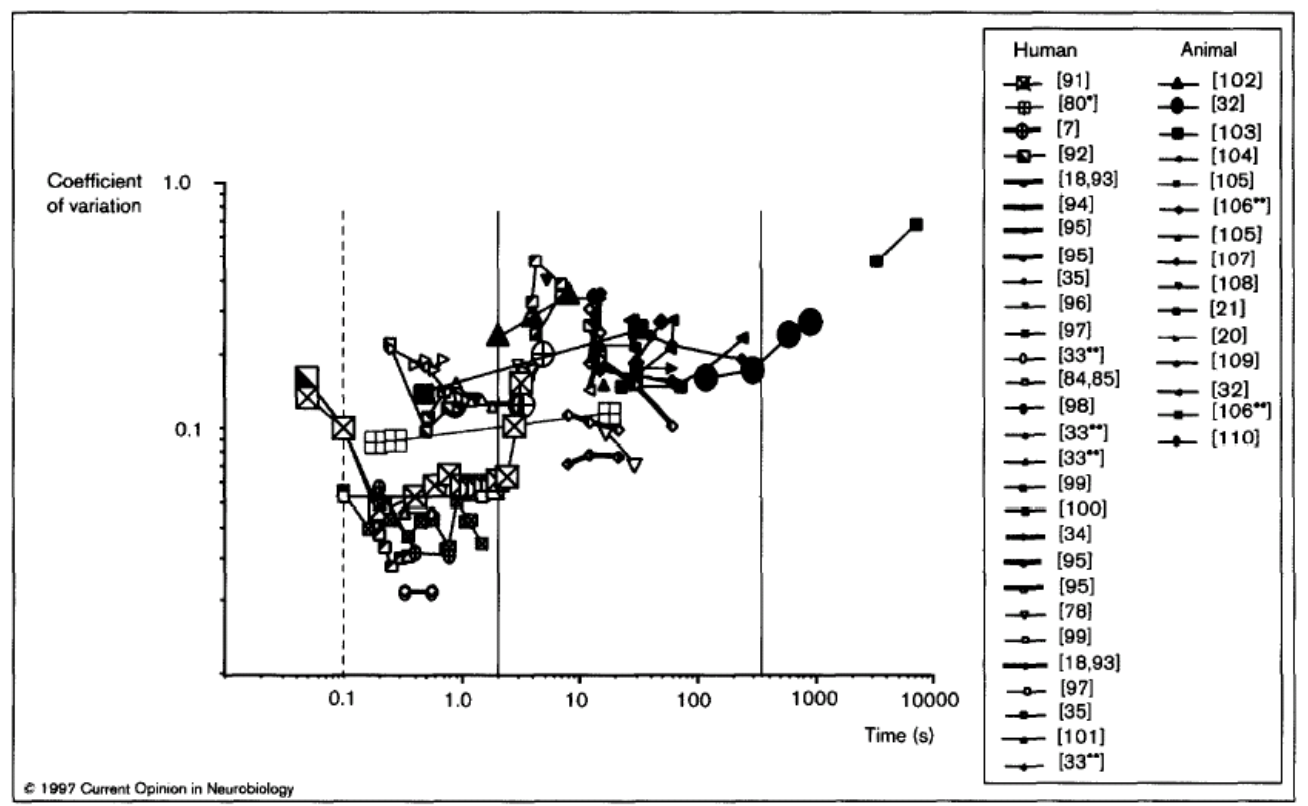

Figure 2.7.: Collection of Weber fractions from a wide range of different studies [58]. The Weber fraction takes the form of the coefficient of variation (Eq. 2.6). Both scales are logarithmic, and the time scale reaches from hundreds of milliseconds to thousands of seconds. The numbers in the legend refer to the citation of each study. Variation between studies is very high, but the Weber fraction roughly decreases at short durations, stays constant at intermediate durations, and increases again at longer and very long durations. These four regimes are separated by horizontal lines. Reprinted from Current Opinion in Neurobiology, 7, J. Gibbon et al., "Toward a neurobiology of temporal cognition: advances and challenges", 170-184., Copyright (1997), with permission from Elsevier.

human studies also support Weber's law [61, 186]. Deviations of Weber's law are, however, also very often reported. These deviations can be put into three categories: (1) Weber fractions that decrease as duration increases, (2) Weber fractions that increase with duration and (3) variability that is independent from duration. The first deviation, decreasing Weber fractions, are often reported at short interval durations below $100 \mathrm{~ms}$. Deviations in this range are readily explained by a straightforward extension of Weber's law, namely allowing for a non-zero offset $b$ in the linear increasing standard deviation $(\sigma)$

$$
\sigma(t)=W t+b .
$$

This relation has been termed the generalized Weber law (see [169], Getty [54] published a similar relation under the same name), and has gained support from a large number of studies [61, 7, 97, 54. The generalized Weber law is currently the most accepted expression of the relationship between variability and duration. However, the other two forms of deviation also 
occur. While constants variability has only been observed in a small number of studies (see [7] for a review), increases in Weber fractions are frequent at longer durations [15, 61, 107]. The duration at which this increase begins varies a lot. For instance, the classical data of Getty [54, which has been frequently cited as reference for the generalized Weber law, shows an increase starting at about $2 \mathrm{sec}$ (Fig. 2.6). Other studies, mainly involving sequences of stimuli, report minimal Weber fractions in the range between 300 and $500 \mathrm{~ms}$, and an increase in Weber fractions at longer durations [39]. These findings can not be easily reconciled with Weber's law.

So to conclude, Weber's law is found to hold at best in a limited range of interval durations. This range largely depends on the details of the respective studies, but as decreasing Weber fractions are most common below $100 \mathrm{~ms}$ and Weber fractions start to increase at durations from $500 \mathrm{~ms}$ [39] to $2 \mathrm{~s}$ [54], the range of durations where Weber's law holds seems to be relatively narrow. Overall, the Weber fraction follows a U-shape rather than a flat line. This form is also reflected in a review by Gibbon and co-workers [58], where he plotted the coefficient of variation for a large range of interval duration. The plot exhibits large variability, but the overall pattern does look much like a U-shape (Fig. [2.7, cf. [15]).

In Section 2.1 .3 and 2.2.3, we will discuss psychological and neuronal models of time perception, respectively, which attempt to incorporate or even explain the origins of both the psychophysical law and Weber's law. In Chapter 3, we develop a neurocomputational model that explains both Weber's law and its deviations for a time scale limited to the sub-second range, and in Chapter 4, we present a more general information-theoretical account for the different behaviors of the Weber fraction.

\subsubsection{Distortion of subjective time by non-temporal factors}

Apart from objective duration itself, temporal estimates have been shown to be influenced by a large number of non-temporal factors [42, 61, 7]. This results both in altered variability and distortions of subjective durations. Here, we review a number of these factors which are relevant for our studies.

Attentional resources. The perception of time has been recurrently found to be modulated by the allocation of cognitive resources such as attention [137, 22, 173, 21]. Drawing resources away from time perception decreases perceived duration and increases variability, whereas additional resources allocated towards time perception have the opposite effect. This has been mainly studies using the oddball paradigm [171, 176, 29] and the dual task paradigm [166, 167, 21, 22. In a dual task experiment, a secondary task is performed concurrently to the time perception task. A number of perceptual, verbal and cognitive tasks have been shown to interfere with time perception. Most consistently, this interference is reflected in a subjective shortening of time, and increasing variability is also frequently reported. In the oddball paradigm, on the other hand, the saliency of a specific stimulus (the oddball) in a series of standards is increased to draw attention to that particular stimulus. This kind of manipulation results in an increased subjective duration of the salient stimulus, and in an increased performance in discriminating it from the standards. 
Effects of task difficulty have also sometime been attributed to resource allocation. An example comes from the bisection task (see [7] or [186] for details) where participants have to classify a stimulus as being closer to a long or a short standard which have both been presented previously. In this task it has been found that the variability of the stimulus representation actually gets lower as the difference between the long and the short standard become smaller, which makes classification more difficult [184, 186]. This was explained with an increased allocation of resources in the more difficult case.

Traditionally, attention and resource allocations were believed to occur only for intervals in the range of seconds [150, 110, 80]. Shorter intervals were considered to be processes in a more automatic way that is inaccessible for cognitive interference. However, this view is challenged by more recent experiments (e.g. [151]), including our own (see Chapter 5), which show that dual tasks also interfere with time perception in the milliseconds range.

Motion and dynamic stimulus change. When visual stimuli are judged according to their duration, they are perceived as longer when they are moving, compared to stationary stimuli [19, 156, 59, 20. This long-know illusion of time has been termed "(subjective) time dilation", and the magnitude of this effect increases with the speed of the motion. More recently, Kanai and co-workers [87] have shown that this illusion also occurs when the stimulus is dynamically changing, while being stationary in space. They also showed that the effect of motion speed could be replaced by the rate of change of the stimulus. Johnson and coworkers 84 performed similar experiments, using stimuli in localized parts of the visual field, but they observed the opposite effect of shorter durations as the frequency of the change increases. All these effects of motion and dynamic stimulus have been observed exclusively for visually presented stimuli so far. In Chapter 5, we show that this illusion may carry over to intervals presented in an auditory domain, providing strong support for an interaction of time perception in different modalities.

Modality. Time perception also differs when the stimuli are presented in different modalities. Consistently, auditory stimuli are found to by judged longer than visual ones, and the temporal variability of time estimates is higher for visual compared to auditory stimuli (see [61] and [7] for reviews). Furthermore, when an interval is marked by two brief stimuli at its beginning and end (an empty interval), variability in time estimates is increased when the two markers are presented in different modalities (e.g. a flash of light to mark the beginning and a brief tone to mark the end of the interval), compared to a condition where both markers are presented in the same modality (these studies are also reviewed in [61] and [7]).

They interaction of simultaneously presented stimuli in different modalities has only rarely been studied. In such a situation, auditory stimuli are also considered as longer then visual ones [181]. For further discussions of these of studies, see Chapter 5 .

Adaptation. The final non-temporal effect to be discussion in this section occurs when a comparison interval is presented within an entire series of isochronous standards. The position of the comparison within such a sequence of intervals affects perception of its duration in two ways. First, when presented at the first position of the sequence, the comparison is judged to be longer than the standards [157, 73, 88, a phenomenon that occurs both in the visual 


\begin{tabular}{c|cc} 
response & \multicolumn{2}{|c}{ stimulus } \\
& $\begin{array}{c}\mathbf{N} \\
\text { (noise only) }\end{array}$ & $\begin{array}{c}\text { S+N } \\
\text { (signal and noise) }\end{array}$ \\
\hline No & $\begin{array}{c}P(\text { No } \mid \mathrm{N}) \\
\text { "Correct rejection" }\end{array}$ & $\begin{array}{c}P(\text { No } \mid \mathrm{S}+\mathrm{N}) \\
\text { "Miss" }\end{array}$ \\
Yes & $\begin{array}{c}P(\text { Yes } \mid \mathrm{N}) \\
\text { "False alarm" }\end{array}$ & $\begin{array}{c}P(\text { Yes } \mid \mathrm{S}+\mathrm{N}) \\
\text { "Hit" }\end{array}$
\end{tabular}

Table 2.1.: Possible combinations of stimuli configuration and responses in a signal detection task, together with the probability for each combination. Participants respond "yes" when they think a stimulus was present, and "no" otherwise. Note that the probabilities for the two responses add up to one for each stimulus condition, i.e. $P(\mathrm{No} \mid \mathrm{N})+P($ Yes $\mid \mathrm{N})=1$ and $P(\mathrm{No} \mid \mathrm{S}+\mathrm{N})+P($ Yes $\mid \mathrm{S}+\mathrm{N})=1$.

and the auditory modality and has been termed "chronostasis" [75]. Second, performance in discriminating of the comparison from the standards or detecting its presence is increased when the comparison is presented at later positions within the sequence, resulting in an increased number of standards presented previous to the comparison (e.g. [11, 39, 78, 123. Evidence for this adaptation effect is mixed, however, as there are also studies which report no such effect [141, 164. In Chapter [6, we present a series of experiments where we control for factors like habituation, neural fatigue and memory effects by using a constant sequence length. While most of this experimental work has been done by our collaborator Stefan Blaschke [16], we also present an information-processing model with accounts for this adaptation.

\subsubsection{Information-processing models of time perception}

The observations reviewed above have led to a multitude of information-processing models of time perception. We use the term information-processing model to differentiate this class of models from neurocomputational or information-theoretical models which are described in Section 2.2 and 2.3, respectively. Information-processing models usually postulate a hierarchy of several processing stages which are described by relatively simple mathematical equations. The aim is to account for the experimental data with a minimal set of assumptions. We describe here the most influential of these models for time perception.

\subsubsection{Signal detection theory}

Signal detection theory (SDT) is a general theory of perception rather than a specific model of time perception. We include it here because we make extensive use of this framework in Chapter 6, and because the pacemaker-accumulator model described in the following section also borrows some aspects of signal detection theory.

In its original form, the theory offers a formal description of the task of detecting a stimulus in the presence of noise [69]. Consider, for instance, an experiment in a dark room where in some trials, a dim flash of light is presented, and in others, the room remains dark, and after each trial, the participant has to report whether he has seen a light or not. Because the visual 


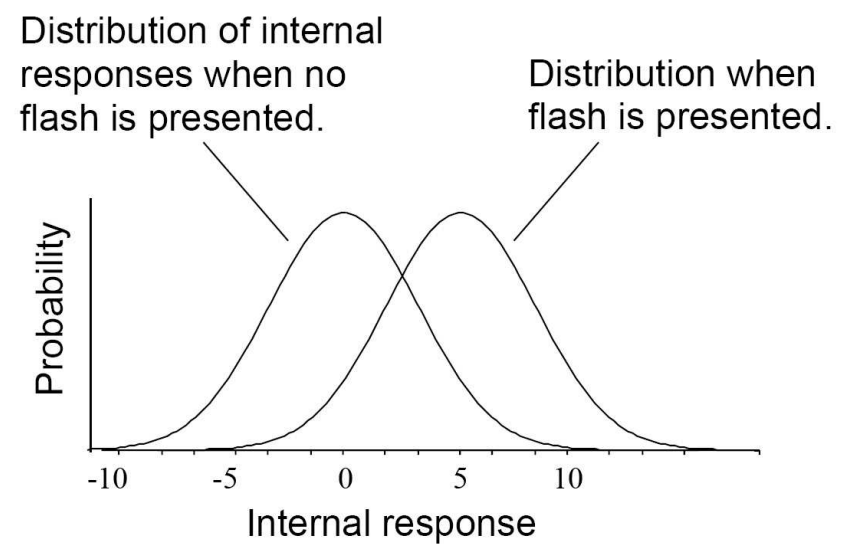

Figure 2.8.: Illustration of the probability distribution of internal responses for the noise-only (left curve) and signal plus noise case (right curve) [69]. Both distributions are Gaussian with identical variance. Note that the distributions overlap to some extent. Reprinted from http://www.cns.nyu.edu/ david/handouts/sdtadvanced.pdf with kind permission from David Heeger

system is not working perfectly and is prone to several sources of noise, you would expect that in some trials, the participant would report to have seen a stimulus although it was not there (called "false alarm"), and in others, he would miss a stimulus that was actually present (called "miss"). In many other trials, however, he can be expected to either correctly detect the stimulus ("hit") or to correctly report that there was none ("correct rejection"). These four outcomes are summarized in Table 2.1. The relative frequencies of each outcome can be seen as estimates of response probabilities. Under the assumptions of SDT, one can use the probabilities $P(\mathrm{Yes} \mid \mathrm{S}+\mathrm{N})$ for a hit and $P($ Yes $\mid \mathrm{N})$ for a false alarm (see Table 2.1), to infer (1) about the variability of the underlying processing system and (2) about a decision criterion $k$ that describes how conservative or progressive the participants acts in detecting the stimulus.

These assumptions of SDT are the following (cf. Fig. 2.8):

1. The stimulus dimension in question (e.g. light intensity, but any other prothetic quantity can be used) can be mapped onto a one-dimensional "internal response" axis with arbitrary units.

2. The statistics of the internal representation of the stimulus can be described by a probability distribution on that axis, centered on the actual value $d$ of the stimulus (biases and other accuracy problems as discussed in Section 2.1.2.1 and 2.1.2.3 are not considered here).

3. In case there is no stimulus, there is also a "noise distribution" with the same variance $\sigma^{2}$ as the stimulus distribution, centered on zero.

4. A stimulus is detected if the value of the internal response in a given trial exceeds a 

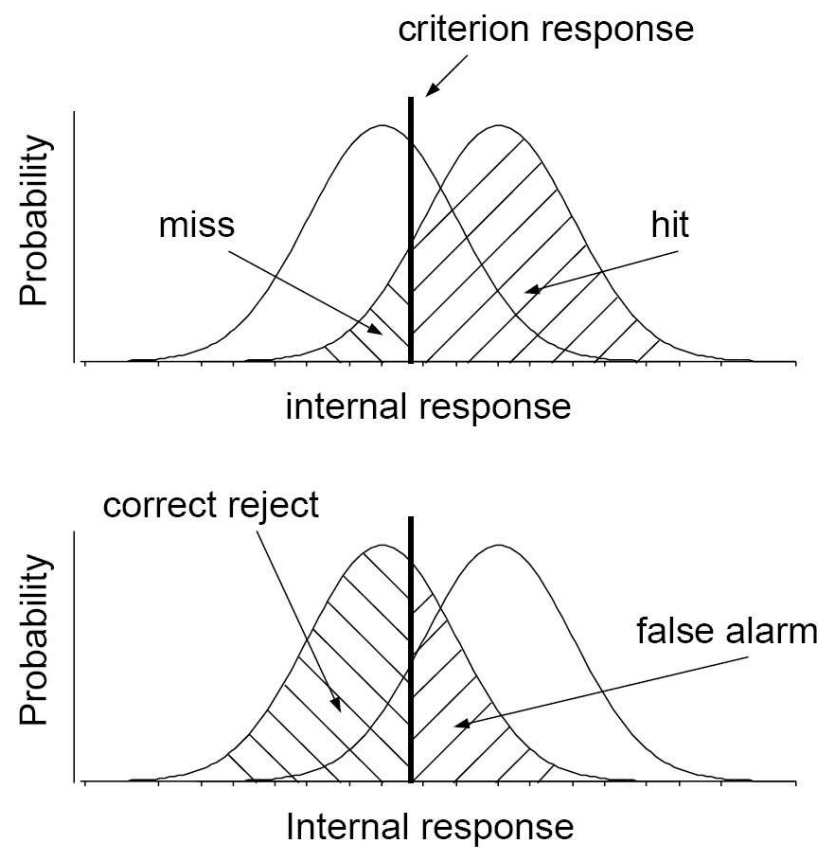

Figure 2.9.: Illustration of the probability distribution of internal responses for the noise-only (left curve) and signal plus noise case (right curve) [69]. The shaded regions are the probabilities for a each of the four outcomes in Table 2.1 in the noise-only case (upper panel) and in the signal plus noise case (lower panel). It is apparent that both the miss and hit probability and the correct rejection and false alarm probability add up to one (cf. Table 2.1). Reprinted from http://www.cns.nyu.edu/ david/handouts/sdtadvanced.pdf with kind permission from David Heeger

certain criterion value $k$, which lies between zero and $d$.

Most often, the PDFs for both the noise and the stimulus distributions are chosen to be Gaussian, $\mathrm{N}\left(\mu, \sigma^{2}\right)$, with mean $\mu$ and standard deviation $\sigma$. The finite variance of the stimulus distribution reflects the uncertainty in mapping the physical stimulus property into an internal representation. The noise distribution, on the other hand, represents the finite probability of reacting to a "phantom stimulus" that was not there, and is also due to the imperfection of the internal representation. Thus, the variances of both distributions are the same. The criterion $k$ adds another degree of freedom, reflecting a trade-off between hit rate and false alarm rate: If $k$ is set to a value close to zero, it is frequently crossed because of random events from the noise distribution, so the false alarm rate is high. On the other hand, the probability that an event from the stimulus distribution falls below the criterion is very low in this situation, so the hit rate is also high (see Fig. 2.9, lower panel). Clearly, the opposite is true when the criterion is set closer to d (Fig. 2.9. upper panel). Thus, a participant may either use a strategy which promises lots of hits, or another one which minimized the number of false alarms. Which strategy he adopts reflects both the relative reward (or punishment) 
of hits and false alarm, and to some degree also general personality features such as shyness or risk-seeking behavior [60].

The above statements can be quantified by calculating the area under each of the corresponding probability distribution functions, as in Fig. 2.9. As we assumed that the variances of the two distributions are the same, we can rescale the axis such that both variances are equal to one. Then, the rate of correct rejections $P(\mathrm{No} \mid \mathrm{N})$ is given by the integral under the noise distribution up to the criterion $k$ :

$$
P(\mathrm{No} \mid \mathrm{N})=\int_{-\infty}^{k} N(0,1)
$$

so $k$ can be inferred from the measured estimate of $P(\mathrm{No} \mid \mathrm{N})$. Likewise, the mean of the scaled signal distribution can be calculated from the area under that distribution that is bounded from below by the criterion value on, which gives the hit rate $P(Y e s \mid \mathrm{S}+\mathrm{N})$ :

$$
P(\mathrm{Yes} \mid \mathrm{S}+\mathrm{N})=\int_{k}^{\infty} N\left(d^{\prime}, 1\right)
$$

We call the mean $d^{\prime}$ because the distribution is scaled by the variance, so that $d^{\prime}$ is given by

$$
d^{\prime}=\frac{d}{\sigma}
$$

As an estimate of $P($ Yes $\mid \mathrm{S}+\mathrm{N})$ is measured from the hit trial and $k$ is inferred from Eq. 2.8, Eq. 2.9 yields $d^{\prime}$, and with the experimentally set $d$, Eq. 2.10 gives the variance of the internal representation $\sigma^{2}$.

Note that this model is not restricted to the paradigm of stimulus detection. For instance, if two stimuli are presented which differ in a certain dimension, the discrimination of these stimuli can be modeled by replacing the noise distribution be the distribution of the stimulus that has the smaller actual value, e.g. the shorter duration. Discrimination is then considered to be accomplished by evaluating the difference $d$ between the two internal response values, and SDT can be applied as described above. This framework will be used in Chapter 6 to evaluate the detection of a deviant interval of prolonged duration in a series of standard durations. There, the SDT framework will also be extended to include distributions of different variances.

\subsubsection{Pacemaker-accumulator models}

The pacemaker-accumulator model (PAM) is by far the most popular modeling framework for time perception, and has been modified and extended in countless ways. In its simplest form, which is due to Creelman [33, this model assumes two components: A pacemaker generating pulses at some frequency $f$, which are counted by an accumulator (also simply called counter). The internal representation of an interval $t$ is then given by the number of pulses counted during the interval $n=f t$. Creelman assumed the pacemaker to be a Poisson process to account for temporal variability. For the discrimination of interval durations, he applied the framework of signal detection theory to the differences between the two intervals (see previous Section 2.1.3). 


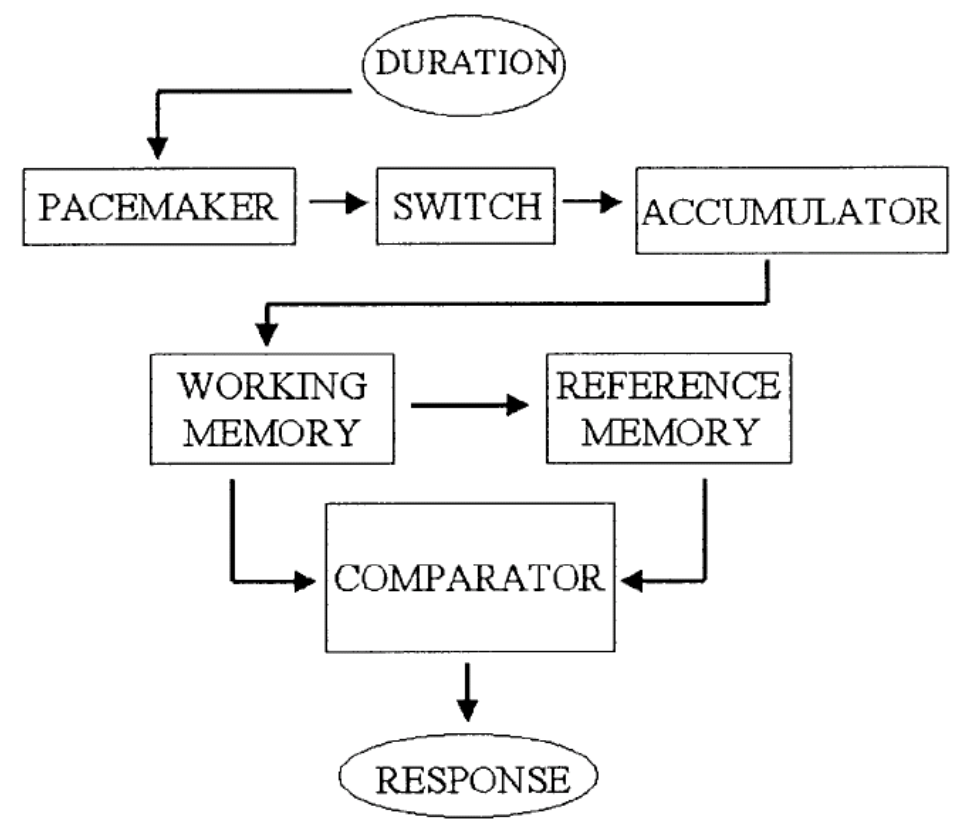

Figure 2.10.: Sketch of the pacemaker-accumulator architecture 8]. See text for details. Reprinted from Behavioural Processes, 44(2), L. Allan, "The influence of the scalar timing model on human timing research", 101-117, Copyright (1992), with permission from Elsevier.

Later, the pacemaker-accumulator model was extended by more explicit modeling of the processes that govern the storage and comparison of the intervals, and which may influence the flow of the generated pulses into the accumulator. Such a more general version of the PAM [8] is shown in Fig. 2.10, Regarding the transmission of pulses to the accumulator, there must be a switch which closes at the beginning of an interval that has to be timed to start accumulation of the pulses, and opens again at the end of that interval to terminate accumulation. In the model of Block and Zakay [18, this switch is also accompanied by a gate (not shown in Fig. 2.10), which may open more or less widely due to different levels of attention, resulting in a larger or smaller number of pulses being accumulated. This allows for a gradual control of the pulse flow, compared to the binary open/closed state of the switch.

After being accumulated, the pulses which represent interval duration are stored in a working memory stage for further processing. This processing frequently involves comparison with earlier presented intervals, that are stored in a reference memory. The comparison itself, finally, is done by a comparator unit.

The reason why all these units are added to the pacemaker-accumulator model is that their function can be formalized by equations, which makes it possible to derive more specific experimental predictions compared to more simple models. Furthermore, each component constitutes a possible point of attack for variability and non-temporal modulation. Indeed, 
the various PAMs mainly differ in a) the nature of the pacemaker-accumulator system, b) the specific stages they explicitly model and c) the assumed sources of temporal variability. Here, we review a representative selection of these models, although it must be noted that not all of explicitly assume a pacemaker-accumulator system. However, all of them can be easily mapped onto a PAM, and we do so to emphasize their similarities.

As mentioned earlier, the earliest PAM [33] assumed a Poisson process with a fixed rate. Because there were no other sources of variability, the standard deviation of the time estimates were predicted to increase with the square root of the interval duration (decreasing Weber fraction). One year later, Treisman published a model with explicit memory and comparator units [169]. His pacemaker was based on a deterministic oscillator, the frequency of which was not fixed, but assumed to vary with the level of general arousal. Divenyi and Danner combined features of these two models [38], assuming a Poisson process with variable frequency, and the possibility to turn the pacemaker on and off (as a functional alternative to the switch). In most later models, the Poisson pacemaker hypothesis was maintained (see [170] for an exception), and the notion of a variable pacemaker frequency has also gained considerable support (e.g. [95, 116. The switch has also been attributed with variability, as well as a latency in its opening and closing [10].

Arguably the most influential among the pacemaker-accumulator variants is Gibbon's scalar expectancy theory (SET), which he developed in the context of reward-based animal experiments [55], and later applied to time perception in humans [57, 56]. He discussed each of stages mentioned above as a potential source of variance, where scalar variance (e.g. variance increasing linearly in time, complying with Weber's law, cf. Section 2.1.2.2) dominates the entire variance. Roughly speaking, Gibbon located the source of this scalar variance in the reference memory and attributed the pacemaker with Poisson and the switch with a constant variability, respectively. Adding all these error sources results in a total variability $\sigma^{2}(T)$ following an equation like

$$
\sigma^{2}(T)=A T^{2}+B T+C
$$

where $\mathrm{A}, \mathrm{B}$ and $\mathrm{C}$ are the variability of the reference memory, the pacemaker and the switch, respectively (see [57] for a more detailed discussion of the different sources of variability).

A strong competitor of SET in the field of animal timing is the Behavioral Theory of Time (termed BeT), developed by Killeen and Fetterman [95, building on earlier work by Killeen and Weiss [97]. Here, the duration representation from the pacemaker-accumulator unit is not directly read out, but used to drive the animal through a series of behavioral states which can be directly observed. For Killeen, the source of scalar variability of the accumulator, which he interprets explicitly as a counter of pulses. The pacemaker and the switch add Poisson and constant variability, respectively, resulting in an equation like Eq. 2.11. The behavioral nature of $\mathrm{BeT}$, and the fact that it does not comprise cognitive stages like memory and comparison may have contributed to the fact that this theory had little impact on studies human time perception. However, Killeen's ideas of optimal counting mechanisms in the presence of various error sources are still influential (see e.g. [96]).

In the following, we briefly discuss how pacemaker-accumulator models address the various non-temporal factors that have been shown to affect time perception (cf. Section 2.1.2.3). Attention has long been modeled by affecting the switch (or the gate in the models of Block 
and Zakay): Attention that is drawn away from a timing task results in a later and more variable closing of the switch [167, 126, 8] or a less wide and also more variable opening of the gate [18]. This general proposal has become known as the attentional allocation model [21] and both possibilities result in a shorter and more variable estimate of duration. However, this model can not account for the fact that subjective duration increases when a salient stimulus draws attention to it, since the total number of pulses is independent of attention. An obvious modification is to allow the pacemaker speed to be modulated by attention [171], which may account for both observed attention effects.

The effect of motion speed and dynamic change can be implemented in a PAM in a very similar way, namely by assuming the pacemaker speed to follow the rate of changes in an environment [48, 146, 20]. Modality effects are also partly accounted for by means of the pacemaker: Visual intervals are processed at a lower pacemaker rate compared to auditory ones. Differences in variability are attributed to the switch, which is assumed to open and close in a more unreliable fashion when visual intervals are involved [142, 185, 174]. Alternatively, separate PAM units may exist for the different modalities, which the above specifications. We further discuss these proposals in Chapter 5 .

Finally, adaptation effects are modeled on the stage of memory. The increasing number of standard presentations prior to the comparison can be explained as an effective averaging of the observed standard durations in the reference memory. Due to this averaging, variability decreases. This notion has been followed by Drake and Botte under the name "multiple look model" [39]. In Chapter 6, we provide a quantitative formalization of that model. Finally, the subjective lengthening of the first stimulus in a sequence has been explained as a change in pacemaker rate, again, presumably as an effect of increased attention at the beginning of a new sequence [75]. This view is supported by the fact that the lengthening effect can be attenuated by training.

In summary, changes in subjective duration are usually attributed to a manipulation of the pacemaker speed, while variability effects are modeled at the stage of the switch or the memory components.

From the above, it is clear that pacemaker-accumulator models provide an extremely flexible framework which can incorporate virtually any feature of time perception. However, this flexibility comes at a high cost: PAM can not really be seen as a scientific theory, as it can hardly be falsified [145]: Any new finding which does not fit into the framework may simply be included by adding another stage or changing or reinterpreting an existent one. An example for this procedure (apart from the above ones) is the reaction of the SET community to findings that violate the scalar properties they built on, the linear increase of both the mean and the standard deviation of time estimates with duration. When faced with the critical deviations from these scalar properties, Wearden 183 included random responses in his SET variant, which terminated the waiting phase prematurely, and were not related to actual time. This resulted in a power law increase of subjective duration in subjective time, and in some cases also increasing Weber fractions at very long durations [107]. Therefore, SET was declared not to be at odds with observations of non-scalar behavior. Also, one can observe a marked bias in the literature by SET supporters in favor of studies reporting scalar behavior (e.g. compare [186] with the present review). 
While attempting to save a theory by modification is a valid endeavor, these examples still illustrate the problem that is posed by the extreme flexibility of pacemaker-accumulator models. Attempts to match the respective units to appropriate functional circuits in the brain may help to constrain pacermaker-accumulator models, and help making more specific predictions.

\subsubsection{Pacemaker-free models}

As discussed above, many theoretical models of time perception can be mapped onto a pacemaker-accumulator architecture. However, there are also some exceptions to this rule. For instance, Staddon and Higa [162] proposed a model that was based on a cascade of decaying memory traces. The time course of the decay, which differs between the traces, is the source of temporal information in this model. Staddon and Higa developed this model as an opponent to Gibbon's scalar expectancy theory. They argued that instead of separating the actual representation of time and its storage into a memory component, it is more parsimonious to consider memory processes as the basis of time perception in the first place. Indeed their model accounts for a number of psychophysical results, including the U-shaped from of the Weber fraction (cf. Section 2.1.2.2).

A second example is given by the dynamic attention model by Jones and co-workers [85, 122, 11, 123. This model is mainly designed to account for the perception of rhythms and sequences of time intervals rather than single intervals. It fundamentally differs from PAMs models in its basic assumptions: The model is built upon a set of oscillators with variable frequencies. An external rhythmic stimulus is represented by means of entrainment of the frequencies. In this way, the oscillators synchronize with the rhythm and constitute an "attentional rhythm" [122]: Any following interval is expected to have the same duration as the ones presented within the rhythm. In this sense, time is represented by means of phase information. Therefore, the model predicts very sensitive responses to distortions in the phase of a rhythm, compared to manipulations that comply with its basic frequency. This prediction was tested with ambigious results (see [16]). In a series of experiments performed by our collaborator Stefan Blaschke [16, we did not confirm this sensitivity on phase information. Therefore, we propose that the representation of sequences of time intervals can be better explained with an extension of the existing PAM architecture, based on the multiple look hypothesis (cf. Chapter 6) [39].

\section{2. (Computational) Neuroscience of Time}

Compared to the progress in the psychology of time perception, there is relatively little definite knowledge about the neural underpinnings of temporal processing. Lesion experiments, imaging studies and electrophysiology in behaving animals have provided lots of data that is compatible with a plethora of timing mechanisms at various brain sites. Likewise, there exists a zoo of theoretical models which show how each of these mechanisms can be exploited to encode time. Here, we provide a brief overview over the neurobiological findings and computational models for temporal processing. We focus on time perception in the range of hundreds 
of milliseconds 4 , with occasional detours to the seconds range, and no reference at all to times shorter than a millisecond or longer than minutes (see [28, 24] for references on these time ranges). We also discuss some basics in the theoretical modeling of biological networks of neurons.

\subsubsection{Brain regions involved in temporal processing}

There are at least three ways of accessing the neural underpinnings of temporal processing. First, one may employ the psychophysical procedures that we discussed in Section 2.1.1, and simultaneously monitor brain activity. This can be done by electrical of magnetical recordings on the scull ( $E E G$ or $M E G$ ), by measuring the blood flow inside the brain which is correlated with neural activity $(f M R I)$, or by tracking certain types of neurotransmitters which are marked by weakly radioactive substances $(P E T)$. The most invasive of these methods is electrophysiology, where electrodes or pipettes of micrometers in diameter are used to record either the voltage of individual neurons (intracellular or single-unit extracellular recording) or the local field potential (LFP) generated by the collective activity of many neurons. All these techniques make it possible to correlate the measured brain activity with the behavioral task. The simplest way to do this is to compare activity measurements in trials containing a time perception task to those with a control task or no task at all. In more sophisticated setups, it is also possible to correlate activity measures with measures of behavioral performance, such as the DL and PSE in a time perception task (cf. Section 2.1.1). However, correlational evidence is always indirect in nature, so even when a certain brain region specifically activates during a time perception task, one can not be entirely certain that this region is functionally relevant for the task.

The second way to assess biological mechanisms of temporal processing is to manipulate certain brain functions and to study their effect on time perception. These manipulations include lesion studies, where certain brain regions are either temporally inactivated or permanently removed and pharmacological studies which alter the neurotransmitter balance in the brain. The field of studying of the effects of neuropathological diseases also broadly belongs to this category5. If any of these manipulations abolishes the sense of time, or effects behavioral performance in a specific way (i.e. impairments of more general functions such as low-level perception or motor response can be excluded), causal evidence is provided that the manipulated brain structure or transmitter system plays an important role in temporal processing. In most cases, however, the manipulation is less specific than desired and also alters the global state of the brain.

Finally, in vitro studies with tissue extracted from animal brains allow to study the biological mechanisms in greater detail. This method provides the highest degree of control over the system, but also at the cost of the farthest deviation from the original situation of the awake, behaving animal. While the relation to behavioral performance can not be studied in such experiments, it is still possible to learn more about specific neural mechanisms that have been identified by one of the above methods.

\footnotetext{
${ }^{4}$ As in Section 2.1, we restrain ourselves to review articles and representative studies, and do not attempt to review the entire literature here.

${ }^{5}$ In animal studies, it is also possible to induce the disease of interest e.g. using genetic mutations.
} 
Note that all the procedures outlined above are not restricted by any means to the investigation of temporal processing, but are routinely used to assess any kind of brain function. But unlike other functions such as spatial vision [89], this rich arsenal of methods so far did not provide evidence for a single mechanism or brain site which is generally accepted as the neural substrate of temporal processing. In imaging studies, a large network of brain regions specifically activates during time perception tasks (Fig. 2.11), and studies of diseases and brains lesions did not find any brain region that disables temporal processing when inactivated [28], although many of these manipulations decrease acuity and increase variability in temporal estimates [28, 24, 81]. Nevertheless, there is a number of brain structures which repeatedly occur in these studies, including the cerebellum, the basal ganglia, and a number of regions in the neocortex such as the Supplemental Motor area (SMA) and the prefrontal cortex (PFC). Additionally, there exist specialized timing systems that are restricted to certain animal species. For instance, neurons in the auditory midbrain of frogs and toads exhibit "temporal tuning curves", or rather frequency tuning curves at low frequencies (about $80 \mathrm{~Hz}$ ). Similar phenomena have been found in bats (review in [121]). Furthermore, the temporal structure of birdsongs seems to be sparsely represented in specific feed-forward structures in the songbird's brain [65. These structures bear similarities with cortical synfire chains [2], which we study in detail in Chapter 3 ,

It is currently strongly debated which brain region contributes to which specific function in temporal processing. Traditionally, it was believed that the cerebellum is responsible for encoding brief intervals in the milliseconds range, while longer intervals in the seconds range were represented in the basal ganglia. However, a large number of more recent studies clearly shows that this dichometry is oversimplified, as we will discuss in the following. To the end of understanding the function of the different brain regions, two studies are particularly worth mentioning. The first is a review about fMRI and PET studies of time perception by Lewis and Miall [110. They categorized the imaging studies according to three dimensions: 1) Whether the task involved a motion component, 2) whether short or long intervals were used (a distinction that is made at about $700 \mathrm{~ms}$ ), and 3) whether continuous and predictable stimuli, such as rhythms, were used. The results of this categorization are shown in Fig. 2.11. Apart from the fact that all of the listed brain regions are specifically active for at least some of the time perception tasks, one can see that the regions on the left side are more often activated in tasks with long intervals and non-motor, discontinuous task, while the regions of the left are more selective for short-interval motor task with continuous stimuli. Based on these task characteristics, Lewis and Miall termed the two classes "automatic" and "cognitive" timing. The brain regions that fall into each of these classes are mentioned in the following paragraphs, but one important result to be mentioned here is that the cerebellum and the basal ganglia do no follow the functions they were classically assigned to: The left lateral cerebellum and the right basal ganglia both fall into the "automatic" cluster, whereas the left basal ganglia and the medial and right lateral cerebellum can not be assigned to any of these classes.

The second study that is interesting in this context is another imaging study by Rao, Mayer and Harrington [154. The authors compared brain activation for an interval discrimination task (1200 ms base duration), and two control tasks. The investigation is of special interest as the authors recorded brain activity by fMRI imaging in four subsequent intervals of 2.5 seconds 

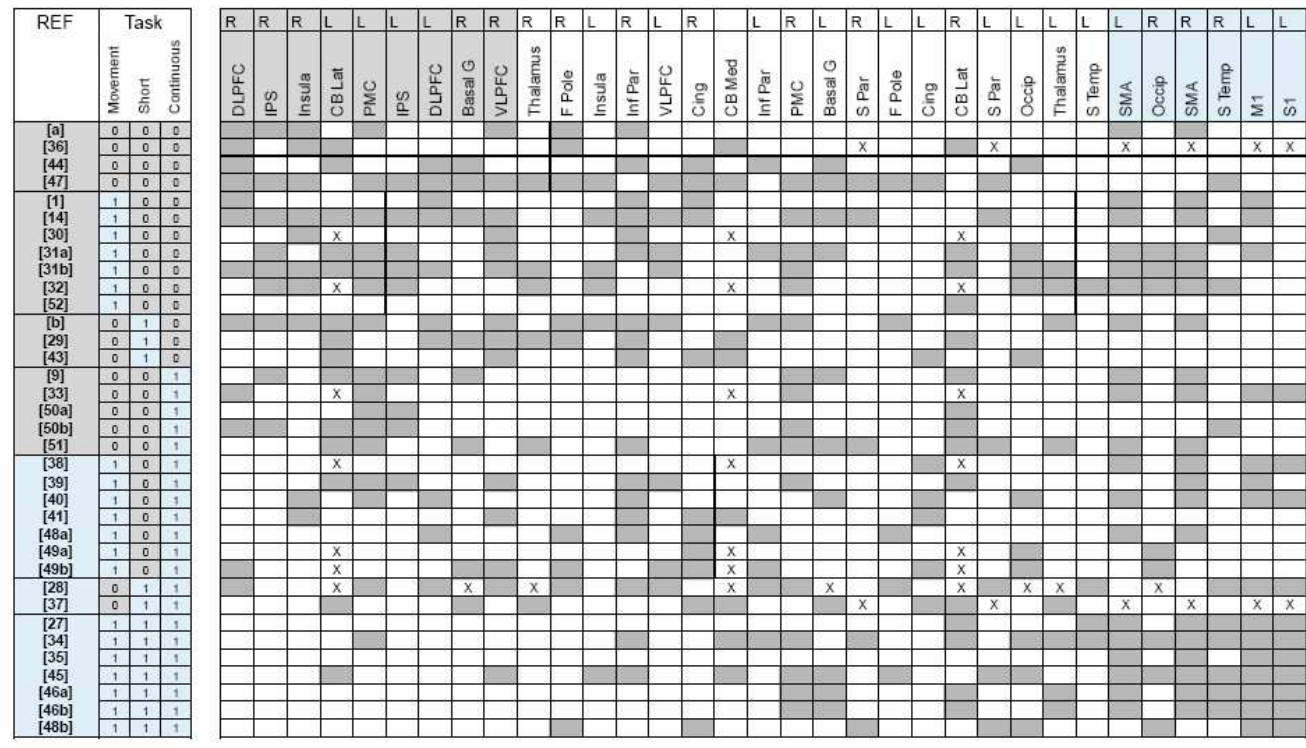

Current Opinion in Neurobiology

Figure 2.11.: Summary figure of recent studies of temporal processing using fMRI or PET imaging from the Lewis and Miall 2003 review [110. The studies are ordered according to three task categories (see text for details). The columns represent the brain regions were specific activity was reported for a time perception task (gray cells), where such specific activity was absent (white cells), or which were not reported ( $\mathrm{x}$ cells) in a given study. The columns are ordered to reflect the affinity of activation of each region to one of the two clusters that are defined by the task dimensions (see text). Reprinted from Current Opinion in Neurobiology, 13, P. A. Lewis and R. C. Miall, "Distinct systems for automatic and cognitively controlled time measurement: evidence from neuroimaging", 250-255, Copyright (2003), with permission from Elsevier. 
each, so they could observed at least the coarse evolution of activity over time. The results showed an early activation (first 2.5 seconds after stimulus presentation) of the left ventral premotor cortex and right putamen, mid-term activation (5 to 7.5 seconds) of the medial caudate, the thalamus, the premotor, prefrontal and temporal cortex, and late activation (10 seconds) of many areas in the basal ganglia, the cerebellum, the right dorsal premotor and prefrontal cortex, and the parietal prenucleus. Furthermore, there was a steady activation of the parietal cortex. From these results, the authors conclude that structures of the basal ganglia, which were activated during the entire time course, plays an important role for time perception, while the lately activated cerebellum is concerned with other tasks then explicit timing. The prefrontal and parietal cortex activation were charged with working memory and attentional processes, respectively, and the thalamus may bridge the interactions between the cortex and the basal ganglia.

\subsubsection{Cerebellum}

The cerebellum is a subcortical structure located at the inferior posterior portion of the head (the hindbrain), and contains about $50 \%$ of the neurons in the central nervous system. The cerebellum is widely accepted to contribute to fine-tuning and feedback control of movement, and to procedural memory and learning [89. In this function, it has first been linked to motor timing [28 and its role in time perception became apparent through clinical and lesion studies (see [77], 58], 81] and [24] for reviews). As these studies were predominantly conducted using intervals in the millisecond range, the notion developed that the cerebellum governed the perception of such short intervals below one second, while the basal ganglia was charged with temporal processing in the seconds range (also called "interval timing", see next section) [79, 77, 24].

However, more thorough reviews of clinical [58] and imaging studies [110, 112] revealed the participation of the cerebellum both in time perception and motor timing for a wide range of durations (Macar [112] specified a range of 0.3 to $20 \mathrm{sec}$ ), although there are individual imaging studies that show no specificity to time perception (e.g. [154]). Lewis and Miall [110] attribute the left lateral cerebellum to cognitive timing, and the medial and right lateral cerebellum to be in between of the two clusters (cf. Fig. 2.11).

Clinical studies consistently show increased variability in time estimates following cerebellar injury [77, 81, but no systematic change in subjective duration [58]. The fact that the scalar property (cf. Section 2.1.2.2) is preserved in cerebellar lesions (as opposed to other lesion sites, see below) has led researchers who are guided by scalar expectancy theory (SET, cf. Section 2.1.3.2) to conclude that the cerebellum is only of marginal importance for time perception, and that the increased variability stems from less specific deficits such as increased motor variability or impaired error correction [58, 24]. To date, the precise role of the cerebellum in general temporal processing is still debated ([154, 113, 121, 80].

Despite of this debate, the cerebellum clearly plays an important role in specific temporal processing tasks. For instance, the cerebellum accurately times the so-called eye blink reflex. In the experimental paradigm of eye blink conditioning, an animal learns to associate a neural stimulus with a an unpleasant air puff on the eyelid, to which it reacts with an blink to 
protect the eye (cf. Capter 3 for more details on the timing of classical conditioning). After lesioning the cerebellum, this reflex is still present, but its precise timing is abolished [143, 99. The cerebellum is also clearly involved in certain forms of motor timing. Cerebellar patients have problems with discontinuous movements, but not with continuous ones, and electrophysiological studies have revealed that cerebellar Purkinje cells in the monkey encode saccade duration in the duration of burst activity [165].

\subsubsection{Basal ganglia}

The basal ganglia is a set of sub-cortical nuclei at the base of the forebrain, including the striatum, the substantia nigra and the nucleus accumbens. These structures are involved in motor control and learning, and in many aspects of cognition and motivation. Specifically, the substantia nigra is one of the main sources of dopamine in the brain, a neurotransmitter which is involved in the encoding of reward and plays a role many neurodegenerative diseases [89]. In the context of temporal processing, the basal ganglia is mostly studied by clinical studies (mostly Parkinson's disease), and by pharmacological manipulations involving dopaminergic drugs.

Parkinson's disease and focal lesions in the basal ganglia have been reported to result in underestimation of short durations (below one second), overestimation of longer durations [58, 24], and increased variability of time estimates [58, 28, 81]. However, this evidence is not without ambiguity, as there are counterexamples for all these phenomena. [58, 81]. Other neurodegenerative diseases of the basal ganglia, such as Huntington's disease and schizophrenia, have similar effects (Fig. 2.12) [24]. The evidence is much clearer regarding lesion studies in rats. After striatal lesions of dopaminergic lesions in the substantia nigra pars compacta, rats are unable to press a lever at the right time to get a reward [119, 129, 24].

As all the abovementioned diseases affect the dopamine balance in the brain, researchers have investigated whether dopaminergic drugs induce similar effects in healthy humans and non-human animals. Indeed, dopamine agonists, such as methamphetamine prolong subjective duration, whereas dopamine antagonists shorten subjective duration in rats [127, 58, 28, 119, 24. In the framework of the pacemaker-accumulator model (cf. Section 2.1.3.2), this would correspond to a speeding-up and slowing-down of the pacemaker, respectively. At least for antagonists, the strength of this effect correlates with the affinity of the drug for the D2 receptor type [127]. The distortion is transient and disappears with training, and there are also rebound effects when the drug administration is stopped (consistent with a clock effect and a slowly adapting criterion in the decision stage) [58, 119]. Similar effects have been observed in humans with dopamine antagonists (see [148] for a review).

Imaging studies have revealed specific activity of the basal ganglia in a wide range of temporal tasks [110, 112]. In most cases, these structures co-activate as part of a cortico-striatal loop (see next section) [153, 112, 130]. According to the categorization of Lewis and Miall [110], the right basal ganglia is involved in cognitive timing, while the left basal ganglia can not be allocated in any of the two clusters.

Finally, electrophysiological studies have reported that activity of spiny neurons in the striatum peaks around times which correspond to trained intervals, which could constitute a direct representation of the the corresponding interval durations [120, 30]. 


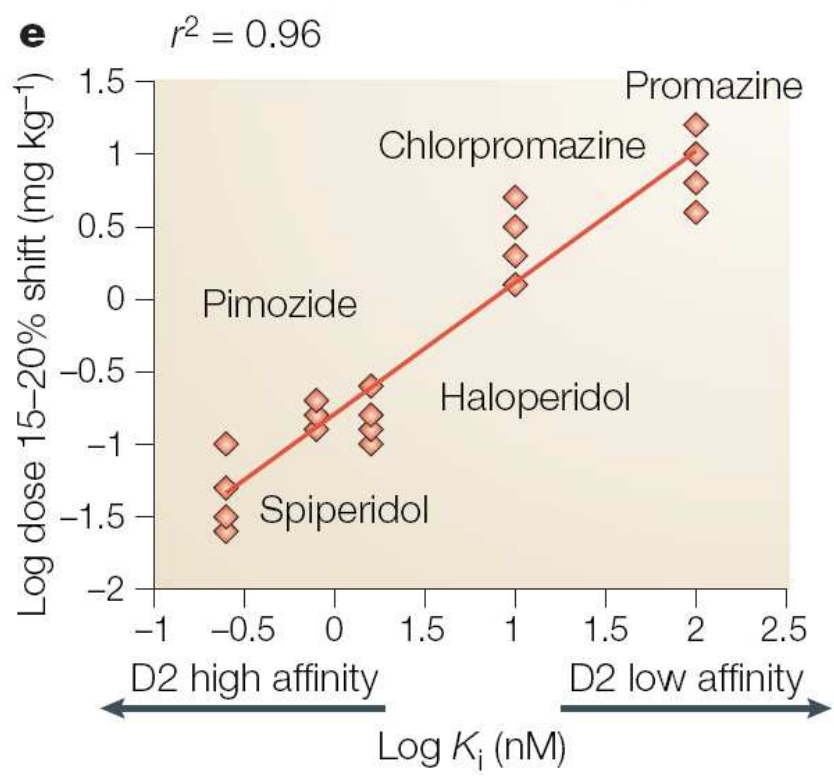

Figure 2.12.: Effect of dopaminergic drugs on time perception in rats as a function of their affinity to the D2 receptor [24]. The y axis shows the dose of each drug that is necessary to induce a 15 to $20 \%$ rightward shift of the psychometric function for a temporal bisection task, which corresponds to a subjective lengthening of subjective time. Reprinted by permission from Macmillan Publishers Ltd: Nature Reviews Neuroscience 6:755-765, C. V. Buhusi and W. H. Meck, What makes us tick? functional and neural mechanisms of interval timing, Copyright (2005)

Classically, the basal ganglia has mostly been charged with time perception in the second range [28, 81]. This view is challenged by the fact that basal ganglia activity is specifically increased for time perception in a wide range of intervals [110, 112], and that clinical studies show impairments of time perception both above and below one second [58]. The dichometry between the cerebellum (subsecond range) and the basal ganglia (seconds range) has been established by Richard Ivry in his influential review in 1996 [77, but eight years later, he concludes himself that "a clear dissociation between the cerebellar and the basal ganglia contributions on temporal processing tasks remains elusive" [81].

\subsubsection{Neocortical structures}

The neocortex is the outermost layer of the cerebral hemispheres, the largest part of the human brain. It can be functionally divided in a number of regions which are mostly responsible for a particular brain functions, such as early vision (primary visual cortex) or motor control (primary motor cortex), although this division is partly artificial. Most knowledge about time perception in the neocortex stems from imaging studies (fMRI, PET and EEG), and electrophysiology. 


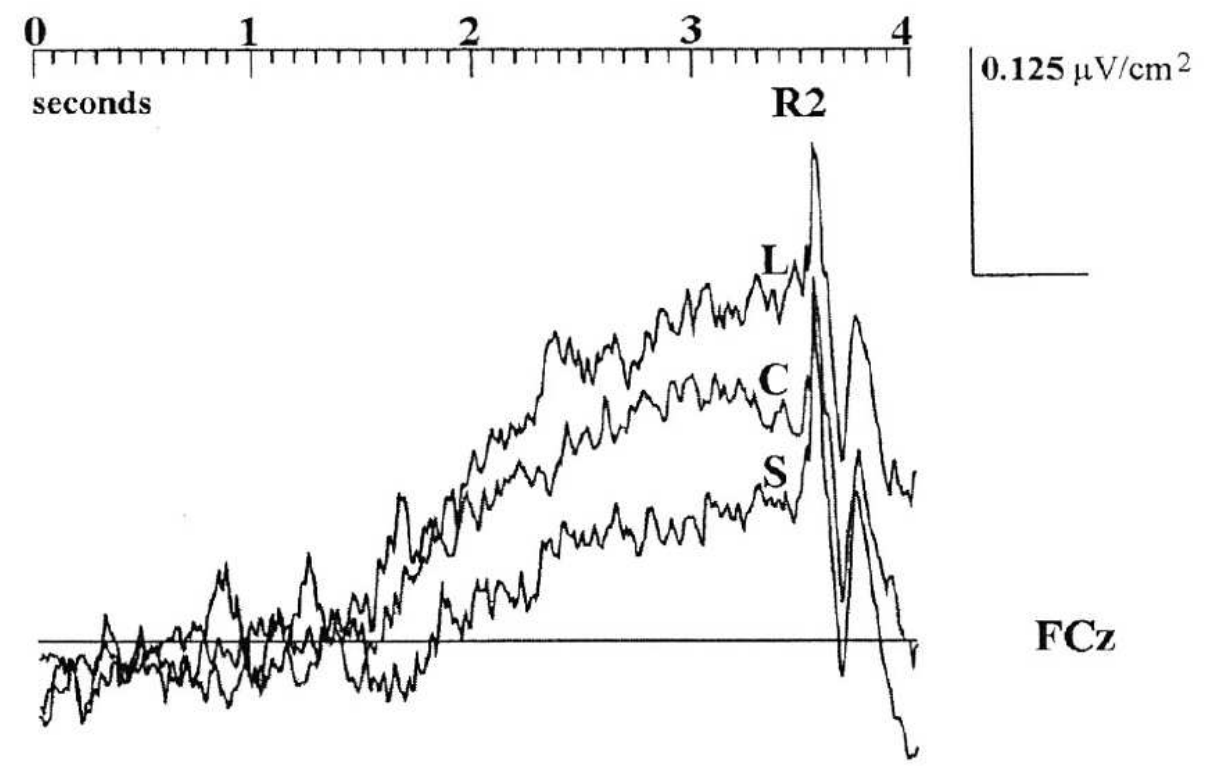

Figure 2.13.: Evolution of the Contingent Negative Variation (CNV) component of EEG recordings [113. The slope of the increasing negativity predicts the behavioral outcome of an interval production task: The three traces are averages over all trials where participants produced intervals that where too long $(\mathrm{L})$, too short (S) or correct (C). Reproduced by permission from the Journal of Neurophysiology, Vol. 18 (2-3), on pp. 89-104, DOI 10.1027/0269-8803.18.2-3.89 (C)2004 Federation of the European Psychophysiology Society.

fMRI and PET imaging typically shows activation of an entire network of structures during time perception, including the prefrontal cortex (PFC), the supplemental motor area (SMA), the premotor, parietal and insula cortex, and the thalamu 6 [153, 154, 112, 110, 130. The SMA and the thalamus are often co-activated together with the striatum, forming a striato-cortical loop (see preceding section) [153, 112, 130]. Lewis and Miall [110] categorize the dorsolateral and the right ventrolateral PFC, the insula and premotor cortex, and the intraparietal succulus as cognitive timing structures, and assign the SMA, the right superior temporal gyrus, the left primary motor and somatosensory cortex as well as the occipital lobe to automatic timing. The parietal cortex can not be allocated in one of the clusters.

EEG studies have focused on a specific component called Contingent Negative Variation $(\mathrm{CNV})$, which is believed to be an index of slowly increasing firing rates ("ramping activity"). $\mathrm{CNV}$ increases during an interval to be estimated, in its slope is correlated with behavioral performance (Fig. 2.12) [114, 113]. The origin of CNV at median fronto-central region (FCz), most likely from the SMA (consistent with the above fMRI studies) [113, 130]. Electrophysiology has revealed populations of neurons in a variety of regions that directly encode interval time by either showing elevated activity around the interval in question (PFC [52], premotor and cingulate cortex [120]), or, more frequently reported, by slowly ramping firing rates that peak

\footnotetext{
${ }^{6}$ which is categorized as a subcortical structure, but mentioned here for completeness
} 
at that time (motor and premotor cortex [105, 158], lateral interpariatal cortex [109], SMA and preSMA [134] and PFC [138]). This ramping activity in single neurons coincides with the increase of the CNV in EEG recordings. In Section 2.2.3, we discuss a model of time perception which is based on this kind of activity.

Less frequently, results of clinical and pharmacological studies of the neocortex are reported with respect to temporal function. Clinical studies report parietal cortex lesions to result in impaired millisecond timing [28, and right PFC lesions to impair interval timing the seconds range [81]. More specifically, temporal estimates are more variable after such injuries, and both short and long intervals are underestimated [58. Furthermore, cholinergic drugs cause a distortion in the memory of estimated durations [128, 58, 24].

Finally, in vitro studies report spikes in cortical slices that occur in reaction to distant stimulation at latencies up to $300 \mathrm{~ms}$ after the stimulation [12, 26]. These findings suggest a functional connectivity within slices which propagates neural activity over times that is much longer than the synaptic transmission times of a few milliseconds.

\subsubsection{Modelling local brain circuits}

Before we discuss some of the neurocomputational models that have been proposed to explain time perception, we briefly review some basics about the modeling of single neurons, synapses and small neural network:7.

Neuron models. The main dynamic variable that is considered in simulation of single neurons is the membrane potential $V$. In the real cell, $V$ is governed by the differences of charged ions inside and outside the membrane of the nerve cell, mainly sodium $\left(\mathrm{Na}^{+}\right)$, potassium $\left(\mathrm{K}^{+}\right)$ and chloride $\left(\mathrm{Cl}^{-}\right)$, and to a lesser extend, calcium $\left(\mathrm{Ca}^{2+}\right)$. The membrane itself is mostly impermeable for these ions, but they can diffuse through small pores in the membrane, the ion channels. The channels are selective for the different kinds of ions, and their permeability may change as another dynamic variable. When ions can freely diffuse through the channels, they settle into a dynamic balance that is given by both the concentration and the electrical gradient over the membrane. Both driving forces are captured by the Nernst equation, which yields the reversal potential $E_{X}$ for each ion $X$

$$
E_{X}=\frac{R T}{z_{e} F} \log \frac{[X]_{\text {in }}}{[X]_{\text {out }}}=\frac{-61.54 \mathrm{mV}}{z_{e}} \log \frac{[X]_{\text {in }}}{[X]_{\text {out }}} .
$$

$R$ and $F$ are the universal gas constant and the Faraday constant, respectively, $T$ is the temperature of the cell, $z_{e}$ the charge of the ion and $[X]_{i n}$ and $[X]_{\text {out }}$ are the concentrations of the ion inside and outside the membrane, respectively. The second equality holds for $T=37^{\circ}$ Celsius, which is the temperature of the human body. The relative concentrations of the mentioned ions inside and outside the membrane lead to approximate reversal potentials of $E_{\mathrm{Na}^{+}}=60 \mathrm{mV}, E_{K^{+}}=-90 \mathrm{mV}, E_{C l^{-}}=-80 \mathrm{mV}$ and $E_{C a^{2+}}=125 \mathrm{mV}$. The resting potential of the entire cell is mostly governed by the $K^{+}$ions, and lies around $-70 \mathrm{mV}$. At

\footnotetext{
${ }^{7}$ These basic facts and modeling approaches can be found in standard textbooks of neuroscience [89] and computational neuroscience [1]
} 
such low voltages, most of the other ion channels are closed. This resting potential can be computed using the Goldman-Hodgkin-Katz equation, which generalizes the Nernst equation to more than one ion and different permeabilities of the respective ion channels.

The dynamic interplay of the ion channels and the membrane potentials can be formalized the Hodgkin-Huxley equation. It is a differential equation which relates the currents across the membrane that are elicited by each of the channels, and the change of the membrane potential which is equal to the sum of all these current 8 . The most general form (also termed Hodgkin-Huxley type model) of this equation reads

$$
\tau \frac{d V}{d t}=\sum_{X} g_{X}\left(V-E_{X}\right)+I_{e x t}
$$

where $g_{X}$ is the conductance and $E_{X}$ the reversal of each ion channel, $\tau$ is the time constant at which the membrane potential relaxes to its net reversal potential, and $I_{\text {ext }}$ combines externally applied currents, as they would be induced by current injection by an experimenter, and synaptic currents (see below). In its original form, Eq. 2.13 comprised only sodium and potassium channels, and a passive "leak channel" to account for current leaks in the membrane

$$
\tau \frac{d V}{d t}=\bar{g}_{L}\left(V-E_{L}\right)+\bar{g}_{K} n^{4}\left(V-E_{K}\right)+\bar{g}_{N a} m^{3} h\left(V-E_{N a}\right)+I_{e x t},
$$

where $L$ stands for the leak current and $n, m$ and $h$ are gating variables with values between 0 and 1 which govern the activation of the $K^{+}$channel and the activation and inactivation of the $\mathrm{Na}^{+}$channel, respectively. The gating variables are both time- and voltage-dependent and constitute an additional set of dynamic variables which are governed by differential equations of the form

$$
\tau_{n}(V) \frac{d n}{d t}=n_{\infty}-n
$$

The voltage dependence of both the steady-state value $n_{\infty}$ and the time constants $\tau_{n}$ can be directly fitted to the results of electrophysiological experiments. At the resting potential, both $m_{\infty}$ and $n_{\infty}$ are close to zero, while $h_{\infty}$ is close to one. As voltage increases due to an excitatory input $I_{e x t}$, both $m_{\infty}$ and $n_{\infty}$ increase, but the activation of the sodium channel has a smaller time scale $\left(\tau_{m}<1 \mathrm{~ms}\right)$ than the activation of the potassium channel $\left(\tau_{n}\right.$ between 2 and $6 \mathrm{~ms}$ ). Thus, the net sodium influx is dominant shortly after the depolarization. If the membrane potential exceeds a certain threshold valu 9 , this triggers a positive feedback loop: Sodium influx increases the membrane potential (as $E_{N a}$ is at a positive value), and the the increasing voltage further opens the sodium channel. Thus, the voltage quickly rises to a large positive value (about $50 \mathrm{mV}$ ). Two mechanisms prevent the membrane potential to remain at this value: First, the slower activation of the potassium channel (with a reversal potential at a negative value) and second, the inactivation of the sodium channel at higher

\footnotetext{
${ }^{8}$ In its single-compartment version, the Hodgkin-Huxley equations does not account for currents within the cell. This spatial extension is introduced by cable theory, which is not discussed here.

${ }^{9}$ Note that this threshold does not necessarily given by a single number. Rather, it is a function of all the dynamic variables in the system. This state-dependence of the threshold is also seen in electrophysiological experiments.
} 
voltages, modeled by a decrease of $h_{\infty}$. Both processes are slower than the activation of the sodium channel ( $\tau_{h}$ between 1 and $9 \mathrm{~ms}$ ), and lead to a decrease of the voltage after the initial increase that was triggered by the positive feedback loop. The result is a short pulse (about $1 \mathrm{~ms}$ ) of positive voltage of uniform shape, called an action potential or spike, followed by a somewhat longer phase of hyperpolarization which prevents spiking at arbitrarily high rates (refractoriness).

For many modeling purposes, the sub-threshold dynamics of the neuron is more important than the detailed modeling of a spike. Recognizing that the active sodium and potassium channels only become relevant when the membrane potential approaches the firing threshold, modelers have proposed simplified models in which the explicit spike generation is not modeled but replaced by a built-in threshold potential: Whenever the voltage crosses this threshold from below, the neuron is considered to spike, and the voltage is set to some reset value (which is typically lower then the resting potential to mimic refractoriness). In its simplest form, the leaky integrate and fire model, only the leak current is explicitly modeled

$$
\tau \frac{d V}{d t}=g_{L}\left(V-E_{L}\right)+I_{e x t}
$$

Without any input, $V$ relaxes exponentially to $E_{L}$, with the time constant $\tau$. This dynamics is sufficient to model temporal integration of synaptic inputs. The simplicity of the integrate and fire model makes it possible to derive analytical statements about its dynamics. On the other hand, many of the dynamical features of the Hodgkin-Huxley model are lost by these simplifications. Some of them, such as bursting, spike-frequency adaptation or rebound spikes following inhibitory input, can be recovered by a slightly more complicated class of models that incorporate a second dynamic variable which mimics e.g. the slow $n$ gating variable for potassium channels. These model 10 are still simple enough to be analyzed by dynamical systems theory (e.g. phase plane analysis), but are suited to model a much broader range of neural phenomena [83].

Modeling synaptic connections. Some ion channels do not react to the membrane potential within the cell, but to the binding of chemical substances from the extracellular medium at so-called receptors outside the cell. This is the way how neurons exchange information via chemical synapses: An action potential in the presynaptic neuron triggers the release of neurotransmitters in the synaptic cleft, which is only about 20 nanometers away from the postsynaptic site, so the transmitter can reliably reach the other neuron via diffusion, and elicit postsynaptic potentials by opening a specific ion channel. The most prominent receptors are the AMPA, GABA and NMDA receptor. AMPA and NMDA receptors both respond to glutamate and selectively transmit sodium, calcium and potassium. With a reversal potential at zero, opening these channels results in a depolarization of the cell, so these receptors are called excitatory. GABA receptors, on the other hand, respond to gammaaminobutyric acid (GABA) and the corresponding channels are selective for cloride, with a negative reversal potential. Thus, opening GABA-dependent channels hyperpolarizes the cell, and this receptor is called inhibitory. Note that the NMDA receptor does not exclusively

\footnotetext{
${ }^{10}$ such as the Morris-Lecar model and the Izhikevich model
} 
depend on the binding of glutamate, but also on the membrane potential of the postsynaptic cell: If the membrane potential is too low, a magnesium block prevents the opening of the channel. Furthermore, postsynaptic potentials elicited by NMDA channels have a much longer time constant compared to AMPA.

These synaptic channels are typically modeled in the same way as voltage-dependent channels, by incorporating another term in Eq. 2.13 for each synaptic input and assuming the conductance of each respective channel to depend on the concentration of a neurotransmitter. The dynamics of this concentration is usually modeled using single- or double-exponential functions, or an alpha function (see Chapter 3), with the time relative to the last spike as the argument. This kind of model accounts for the experimental observation that postsynaptic potentials quickly rise and decay again after a spike from a presynaptic cell has arrived. More sophisticated models of synaptic conductances treat the transmitter concentration as an additional dynamic variable that is governed by a differential equation. In this way, dynamic phenomena such as short-term synaptic plasticity (cf. Section 2.2.3) can be incorporated into the model.

Connectivity and synaptic plasticity. The models of time perception we discuss in the following section are based on small networks of neurons with neocortical properties. While the exact connectivity of neurons in these networks is not known, local circuits are well modeled as randomly connected networks, with about $80 \%$ of excitatory "pyramidal cells" and $20 \%$ of inhibitory "interneurons". Interneurons fire at higher spontaneous frequencies compared to pyramidal cells, so there is a balance of net excitation and inhibition in such a network, leading to a high variability in the sub-threshold membrane potential in a given cell, which is of the same order as the mean of the potential. Cortical networks are most often recurrently connected, meaning that it is possible to find directed loops in the connections, so that the network can not be segregated into layers which transfer neural activity from one layer to the next. However, it is possible that such layered structures, called feed-forward networks, are embedded in a larger recurrent network. In the limit case that the feed-forward network is much smaller than recurrent network, one can treat the recurrent network as a source of stochastic input to the embedded feed-forward network, neglecting the effects of the feed-forward onto the recurrent network. This case is considered in Chapter 3 for a specific feed-forward network, the synfire chain [2] (see below).

The efficiency of synaptic connections between the cells of a cortical network can be modified by activity-dependent synaptic plasticity. Many of these modifications are of the Hebbian type, meaning that an increase of synaptic efficiency requires both the pre- and the postsynaptic cell to depolarize within a short time window (or, more concisely, "cells that fire together, wire together"). A candidate neural mechanism for this kind of plasticity is the NMDA-mediated calcium influx into a cell, which is believed to potentiate the efficiency of AMPA receptors. As the NMDA receptor needs both glutamate binding (elicited by the firing of the presynaptic cell) and depolarization of the postsynaptic cell, this receptor fulfills the basic requirements for Hebbian learning. A more specific form of Hebbian plasticity is spike-timing dependent plasticity (STDP). As the name suggests, not only the coincidence of spikes in the pre- and postsynaptic cell is important for this kind of plasticity, but also the relative timing of the 
spikes. We elaborate on STDP in Chapter 3, where we show how this kind of plasticity can select the optimal input to a discrimination network based on the spike time variability of the inputs.

Synfire chains. A particular neural architecture that is frequently used as a model for the cortical microcolumn [89] is the synfire chain [2]. It is a feed-forward network consisting of many layers (or pools) with strongly converging and diverging connectivity from one pool to the next, i.e. each neuron receives activity from many neurons in the preceding pool and also projects onto many neurons in the next pool. This type of connectivity favors synchronous firing within each pool: It has been shown that activity that is injected into the first pool of synfire chains stably propagates through the entire chain, and that the temporal jitter of the spike times within each pool converges to a fixed point as values below one millisecond [70, 37]. This synchronous transmission is maintained for a wide range of parameters and initial condition of the model. Theoretical considerations have shown that the synfire architecture is well suited for the storage of stimulus patterns [70, which makes it a favorable model for cortical computations. It was also proposed as the neural substrate of binding different stimulus features together by means of weak synchronization of different chains [68, and for a precise temporal code (see below).

It is an experimental hard task to observe a synfire chains in the brain, as the pools are not necessarily spatially organized, so the current multi-electrode recordings of about 100 neurons at a time are likely to capture a only very few neurons within the same chain. However, indirect evidence comes from the observation of precisely timed spike patterns (see [2, 3, 4, 63, for reviews): Statistical analysis of recorded spikes shows reliable spatio-temporal patterns with a precision that is hard to be explained with any model except a synfire chain. However, these results have also been challenged by the argument that these patterns may also occur by chance, and that the null hypotheses of the statistical tests of pattern significance may be flawed ([124], see also [3]). Thus, the existence of synfire chains, or rather their necessity to explain precise spike patterns, is debated (see [3] and [63] for current arguments). Nevertheless, it is suggestive to study synfire chains in the context of temporal processing, as their favorable temporal precision make these structures idealy suited for such a task. In Chapter 3, we analyze the synfire chain model in more detail and show how it can be used as the basis of neural model of time perception that complies with psychophysical observations.

\subsubsection{Computational models of time perception}

Based on the neurobiological findings we reviewed above, theoretical neuroscientists have attempted to formulate models that are capable of representing temporal information. Virtually any process in the brain that evolves in time could be used for this purpose. Consequently, there exists many computational models of time perception which exploit a wide range of these mechanisms (see [77, 58, 28, 121, 119, 24] and Chapter 3 for reviews).

A plausible model of temporal processing is not only required to be based on neurobiological findings, but also to reproduce as many of the psychophysical phenomena as possible (cf. Section 2.1). Unfortunately, many neurobiological models do not make predictions about psychophysical experiments, but are exclusively designed as a mechanistic account for temporal processing. An exception is the scalar property of temporal variability (cf. Section 2.1.2.2), 
which has been attempted to be explained by a number of models (cf. Chapter 3).

Here, we present three of the mostly discussed models to date, all of which are based on neurobiological findings. Only one of them is directly concerned with reproducing a psychophysical observation, the scalar property, but it does so by ad hoc assumptions rather than generic properties of the model. In Chapter 3, we discuss a model from with the more complex, U-shaped Weber fraction of the timing error emerges.

State-dependent networks. The first of these model has been proposed by Dean Buonomano and coworkers [25, 28, 90. This model assumes that time is encoded in spatio-temporal patterns of activity generated by cortical networks. The main time-dependent processes in the model are short-term synaptic plasticity and slow inhibitory synaptic currents (modeling $\mathrm{GABA}_{b}$ receptor dependent ion channels) 11 are used. Paired-pulse facilitation (PPF) increases the excitatory postsynaptic potential (EPSP) elicited by an excitatory input that occurs within a certain time after a previous stimulation. This amplification peaks at about $50 \mathrm{~ms}$ and decreases to baseline about $300 \mathrm{~ms}$ after the first input. Conversely, paired-pulse depression (PPD) decreases the strength of an inhibitory postsynaptic potential (IPSP) elicited by an inhibitory neuron that has fired before. The depression is strongest at $200 \mathrm{~ms}$ after the first input and decreases to baseline after about $400 \mathrm{~ms}$. Finally, the slow synaptic input from inhibitory neurons follows the same time course as paired-pulse depression.

In its simplest form, the model comprises only two neurons, an excitatory and an inhibitory one (called $E$ and $I$ in the following), and five synapses (Fig. 2.14, upper left panel): An excitatory synapse provides external input to both neurons, $I$ projects to both itself and the $E$ via a slow $\mathrm{GABA}_{b}$ synapse, and additionally, to $E$ via a fast $\mathrm{GABA}_{a}$ synapse (with a time course that is negligible compared to the slower time scales). To test for interval selectivity, these neurons are stimulated both at the beginning and the end of an interval of varying length. Because the synaptic delay of the input to $I$ is shorter compared to $E$, only $I$ fires in response to the first stimulus, cutting off the spike of $E$. During the interval between the two stimulations, the slow processes change the state of this small network and determine the strength that is needed to activate both neurons as a function of time. In this simple example, this is most easily seen if one considers PPF and the slow IPSP alone, which govern the excitability of both neurons alike: Around $50 \mathrm{~ms}$ after the first pulse, PPF is dominant over the slow IPSP, so even a small input will make the neuron fire. At later times, however, PPF decays and the slow IPSP becomes dominant, so stronger input is needed to explicit a spike. In this way, the longer the interval between the two pulses, the higher the second input must be to be effective. In this setup, the $E$ can be easily made selective for an interval $T$ by tuning the weights of the input to both neurons: To prevent $E$ from firing too late, its input weight is set such that it can only fire up to time $T$. And to prevent $E$ from firing too early, the input weight to $I$ is set such that it can fire up to a time slightly before the $T$. This will cut off the firing of $E$ at all times before $T$ because of the fast GABA $_{a}$ inhibition. In effect, only a narrow window of intervals around $T$ remains at which $E$ can fire, so $E$ is made selective for the interval $T$. Fig. 2.14 shows how the weights must be chosen to make $E$ selective for three different intervals (lower panel), and how the two neurons react to the

\footnotetext{
${ }^{11}$ All of the mechanisms and parameters used in the model are based on neurobiological findings in cortical neurons.
} 


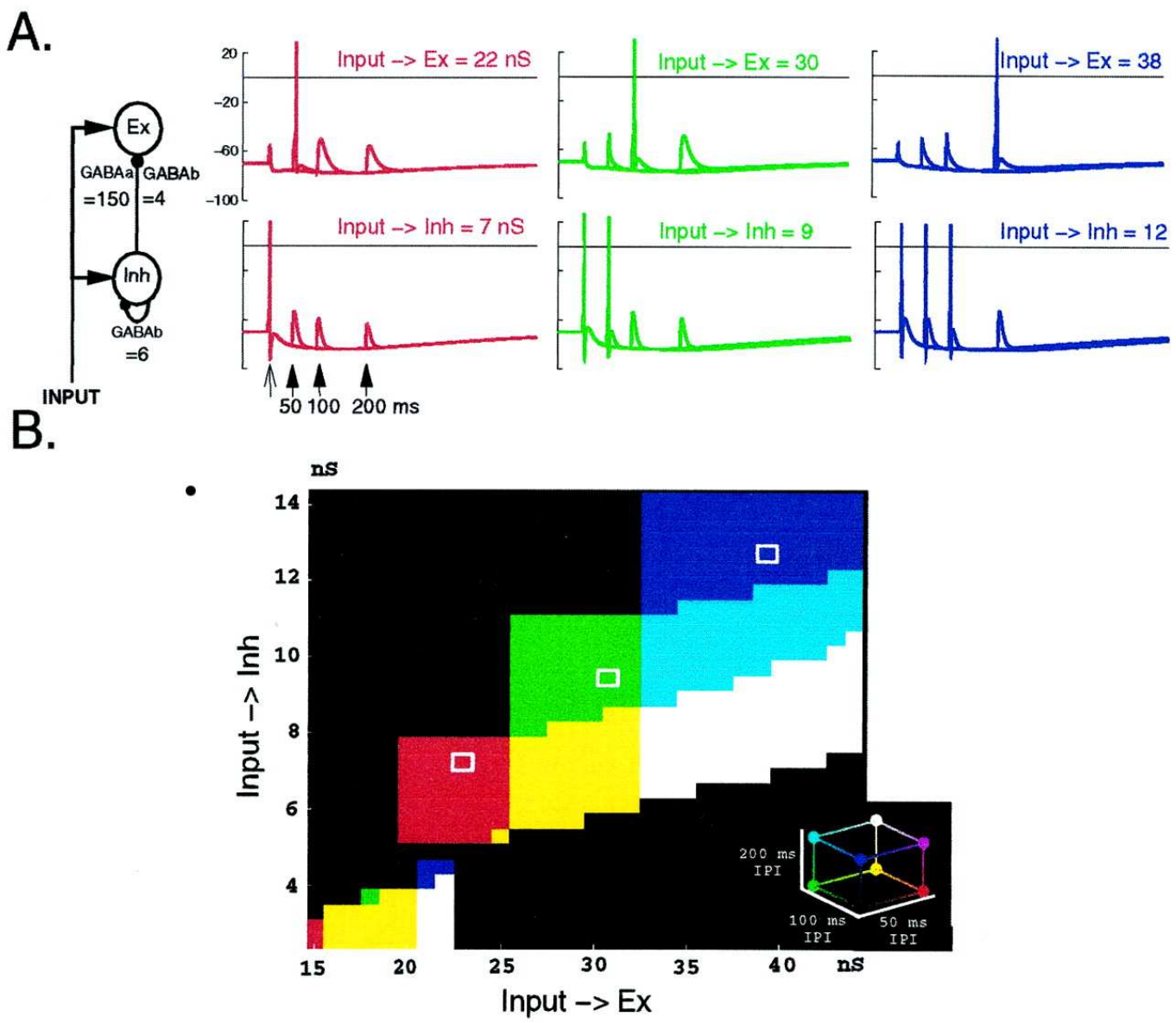

Figure 2.14.: Two-neuron state-dependent network that is capable of interval discrimination [25]. Upper left panel: The circuit consists of an excitatory $(E)$ and an inhibitory neuron $(I)$ and five synapses, two excitatory ones from external input and three inhibitory ones which project from the $I$ neuron onto itself and onto the $E$ neuron (see text for details). Upper right panel: Membrane potential of the $E$ (upper traces) and $I$ neuron (lower traces) as a function of time. The neurons respond to an initial stimulation (open arrow) and a second stimulus 50, 100 or $200 \mathrm{~ms}$ after the first one (closed arrows). The responses for all three cases are overlaid. From left to right, three different configurations of synaptic weights are shown, which lead to selective responses of the $E$ neuron to one of the three intervals. Lower panel: Parameter space of the two variable synaptic weights. The color represents the intervals to which the $E$ neuron is selective (see colour legend at lower right). Larger input to the $E$ neuron make it respond to longer intervals, while larger input to the $I$ neuron prevents responses to earlier intervals. Reproduced from the Journal of Neuroscience 20(3):1129-1141, D. V. Buonomano, "Decoding temporal information: A model based on short-term synaptic plasticity" with permission from the Society for Neuroscience, Copyright (2000). 
inputs at these times (upper right panel).

To show that interval selectivity can emerge without fine-tuning of parameters, Buonomano uses a larger network of 400 excitatory and 100 inhibitory neurons with random connections of low probability, and synaptic weights drawn from a Gaussian distribution. In this larger network, the individual neurons are no longer selective for a specific interval. However, when the overall activity of the network is monitored by a discrimination network that is trained to associate the activity of the network with one out of six interval durations between 50 and 300 ms by supervised learning, this discrimination is successful, and also generalizes to intervals that are in between of the trained ones, leading to gradually decreased responses. This shows that the network as a whole contains the temporal information, even in the presence of synaptic noise. This resembles the kind of computation performed by a liquid-state machine [111.

Note that the temporal range of the model is limited by the time constants of the slow processes to intervals up to 200-300 ms. Intervals above this range must be represented by other mechanisms, which are not specified in the model. If the model implemented in the brain, this limited range may be the cause for the separations some researchers observe between long and short intervals, although both this separation and the interval length at which it may occur are debated (cf. Section 2.1.2.3 and 2.2.1). Apart from explaining this separation, the model does not address any of the psychological effects reviewed in Section 2.1. Instead, it predicts one of its own, namely the context-sensitivity of duration estimates. According to the model, the representation of a given interval depends on the state the network was in at the beginning of that interval. Thus, if another interval was presented shortly before, this should influence the representation of the second interval. In a recent psychophysical study, this effect seems to be confirmed [90. Because of this context-sensitivity, the model is equally well suited to account for the representation of sequences, and for order judgments [25]. This flexibility, and the fact that does not rely on mechanisms that are specialized for the perception of time, make the model interesting, despite of its currently limited account for psychophysical effects.

Self-organized climbing activity. The second model also focuses on time perception in the neocortex, but assumes that temporal estimates are realized in the form of slowly increasing firing rates during the interval to be encoded. This kind of activity is frequently observed in cortical recordings (cf. Section 2.2.1.3). Daniel Durstewitz has proposed a model for the neural basis of ramping activity that is based on the dynamics of single neurons with cortical properties [40]. According to the model, the dynamical basis of ramping activity is a positive feedback loop between the firing rate of the neuron, and spike-based calcium influx. The neuron is modeled as an integrate and fire unit with an after-hyperpolarizing (AHP) current, which ensures a realistic refractoriness period, and a calcium-dependent after-depolarizing (ADP) current. The ADP current is triggered by calcium influx into the neuron after each spike, and can be seen as a source of input into the neuron: The higher the firing rate of the neuron, the higher the calcium influx, and thus the depolarization after each spike. This positive feedback loop generates persistent activity if there is a sufficiently strong initial input to the neuron 12. Ramping activity is produced in the model when the system is in a

\footnotetext{
${ }^{12}$ Input is modeled both by synaptic currents resembling AMPA, NMDA and GABA receptor dependent ion channels, and by direct electrical stimulation
} 

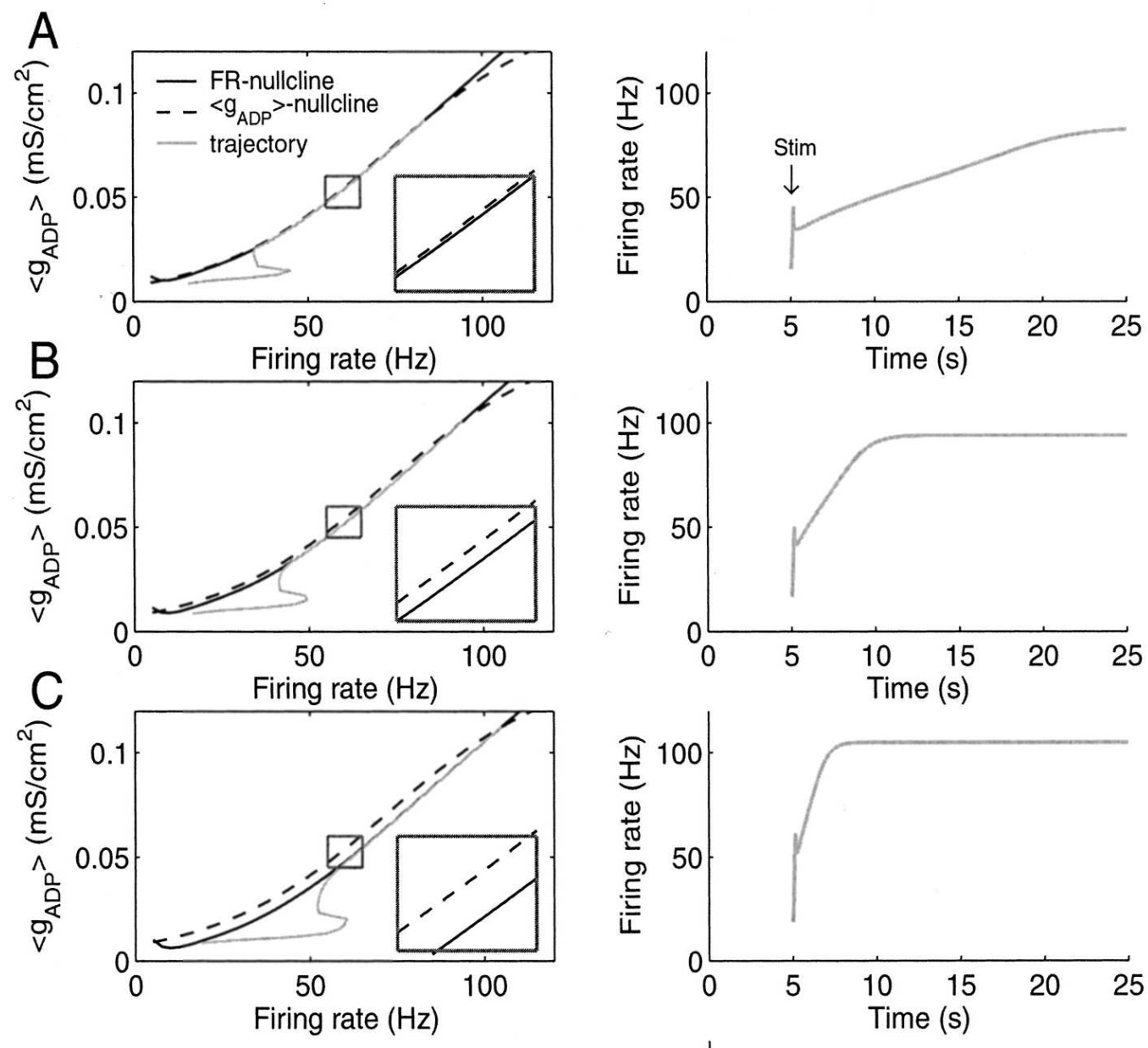

Figure 2.15.: Illustration of the dyanamics in a model of ramping activity [40] (see text for details). Left panels: Nullclines in the $f$ and $\langle A D P\rangle$ phase space for three different parameter configurations which lead to increasing separation of the two parallel nullclines. Right panels: Ramping firing rates for these three configurations. The slope of the ramping becomes steeper as the two nullclines move further apart. Reproduced from the Journal of Neuroscience 23(12):5342-5353, D. Durstewitz, "Self-Organizing Neural Integrator Predicts Interval Times through Climbing Activity" with permission from the Society for Neuroscience, Copyright (2003). 
configuration close to a line attractor [182, 117], in which the transition to the high-rate fixed point is slow. This can be seen by looking at the phase portrait of the model in the space of the instantaneous firing rate $f$ and $\langle A D P\rangle$, the ADP current averaged over one inter-spike interval $\left(T_{I S I}=1 / f\right)$. In this phase space, one can compute the $f$ nullcline, which represents the average amount of ADP input necessary to maintain a given firing rate $f$, and the $\langle A D P\rangle$ nullcline, which is the amount of ADP that is produced at a given firing rate $f$ (Fig. 2.15). Where these nullclines intersect, demand and supply of ADP is in balance, so there is a fixed point of the system, which is stable if the slope of the $\langle A D P\rangle$ nullcline is no steeper than the slope of the $f$ nullcline. Thus, if the two nullclines lie on top of each other, they constitute a line attractor, a series of stable fixed points at a range of values of $f$ and $\langle A D P\rangle$. Ramping activity now arises at configurations where the nullclines are very close to a line attractor: Because of the mismatch between the nullclines, both firing rate and the average ADP current move towards the fixed point. But when the mismatch is small, this transient phase can be quite long, because the system state changes slowly in the vicinity of what is called the "ghost of a line attractor". In this way, different ramping speeds can be achieved by changing the mismatch between the nullclines (cf. Fig. 2.15). The can be regulated by the amount of recurrent synaptic input into the neuron: The higher this input, the lower the ADP input needed to maintain a given rate, so the $f$ nullclines shifts down parallely to its initial position. This is exactly how the different panels in Fig. 2.15 were generated [40], and in a real neuron, this can be achieved by means of synaptic plasticity.

The question remains how the parameters of a real neuron could be adjusted such that the two nullclines are almost in parallel over a wide range of firing rates, which is a prerequisite for ramping activity. Durstewitz proposes that this could be achieved by a self-organization process that monitors long-term fluctuations of the calcium concentration: In the line-attractor configuration, transients are long and pronounced and thus, also the reaction of the system to synaptic noise is strongest. So, the system can drive itself into a line attractor configuration adjusting its parameters (here, the steepness of the $\langle A D P\rangle$ nullcline) such that the variance in the calcium concentration is maximized.

Regarding the possible ranges on durations, the model could in principle represent arbitrary long intervals, as the transition to the fixed point can be made arbitrarily slow when approaching the line attractor further and further. Still, it is hard to predict to what precision the self-organizing process could actually adjust the system. On the other hand, while a large separation of the nullclines results in a very fast transient, there is probably a lower bound that is given by the intrinsic time constants in the model. So, although not explicitly discussed in the paper, the model seems to be best suited for relatively long interval between several hundred milliseconds 13 and several seconds.

In its original form, the model does not address any of the psychophysical phenomena discussed in Section 2.1. However, it has recently been shown [161] that the scalar property (cf. Section 2.1.2.2) could emerge from a more simplified ramping activity model, when combined with a set of readout neurons, and some further assumptions. Furthermore, we recently

\footnotetext{
${ }^{13}$ The longest relevant time scale in the model is about $100 \mathrm{~ms} 40$
} 
re-analyzed Durstewitz' model and preliminary results show that its representation of duration shows sensitivity to non-temporal features such as stimulus intensity (cf. Section 2.1.2.3). Furthermore, we could reproduce the distortion in subjective duration induced by the D2 dopamine receptor (cf. Section 2.2.1.2). These results and its biological plausibility make the climbing activity model a promising candidate for the representation of intervals in the seconds range.

Striatal beat model. The final model to be discussed in this section also assigns a role of the neocortex for time perception, but puts its emphasis on the interaction between cortical and striatal networks, in accordance with neuroimaging results (cf. Section 2.2.1.2 and 2.2.1.3).

It has been proposed by Matell and Meck [119], based on earlier work by Miall [133]. In this model, the representation of time is based on cortical oscillators of different frequencies, which are read out by converging projections to striatal spiny neurons (Fig. 2.16). If the oscillators differ only slightly in frequency, the sum of their activity exhibits beats of much larger periods than the original oscillations. In this way, oscillators with frequencies in a biological range (5-15 Hz) are capable of representing intervals of much longer duration (up to 20 seconds).

While these oscillations could be represented by periodically changing firing rates, the actual simulation was performed using simple cosine oscillators which represent the membrane potential of individual neurons. To generate spikes, a threshold was introduced which declared a neuron as spiking whenever the membrane potential was above this threshold. This model resembles a very simple version of the integrate and fire neuron which is known as the theta neuron [44], with the difference that these oscillators were considered as spiking (or bursting) during a finite fraction of their oscillation, and not at a single phase as the theta neuron. The original simulations by Christopher Miall [133] also comprised an output neuron where the oscillators project to, with synaptic strengths that were adjusted according to a Hebbian learning rule such that a specific criterion time was stored. As a result, the output neuron fired at a high frequency at that specific criterion time. Matthew Matell and Warren Meck generalized these simulation by incorporating noise into the model and studied its effects on the timing errors to compare the simulation with the performance measures obtained in psychophysical experiments (cf. Section 2.1). Specifically, they introduced global variability in the frequencies of the oscillators, and set the synaptic connections as they would result from repeating exposure to a joint input following a Gaussian distribution with a standard deviation that scales with the criterion time. Together with a number of other extensions, the model reproduces the scalar property, i.e. the distributions of spikes in the output neurons superimpose when scaled by the base duration (cf. Section 2.1.2.2). Of course, this is a direct consequence of the scalar variance that was introduced at the level of the oscillator frequencies and the synaptic weights.

Matell and Meck conceptually embedded this model in a review of neurobiological findings by interpreting the convergence site as the striatal spiny neurons, showing that their anatomical and electrophysiological properties are well suited for coincidence detection of cortical oscillations. Furthermore, they hypothesized that phasic bursts of dopamine from the substantia nigra pars compacta served both as a learning signal to associate certain patterns of 

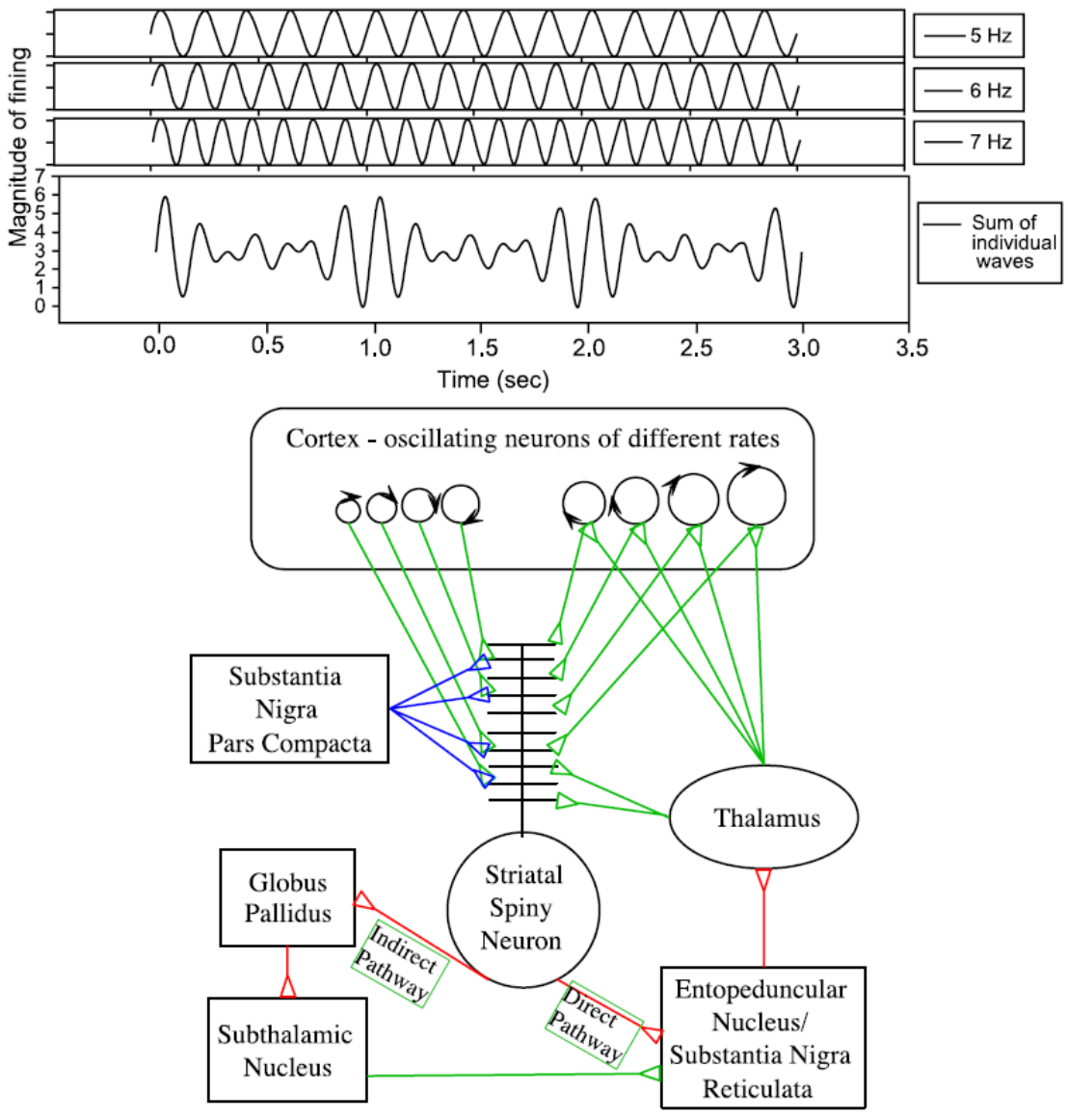

Figure 2.16.: Basic structure of the striatal beat model [119]. Upper panels: Neural activity of three oscillators with different frequencies (without threshold) and the sum of these three oscillations, which exhibits beat at much longer periods compared to the individual oscillators. Lower panel: Neural instantiation of the components of the model. The oscillators are located in the cortex and their activity is read out by striatal spiny neurons, which are further influenced by dopaminergic modulation (substantia nigra) and indirect pathways which enable associative learning of the oscillator pattern that correspond to a given interval. Reprinted from Cognitive Brain Research, 21, M. S. Matell and W. H. Meck, "Cortico-striatal circuits and interval timing: coincidence detection of oscillatory processes", 139170, Copyright (2004), with permission from Elsevier. 
cortical activity with specific intervals, and as a "starting gun" which resets the phase of the oscillator: 14 .

This model is currently very popular (see e.g. [24, 130]), as it strives to combine neurobiological and psychophysical findings. However, it must be emphasized that the scalar property does not emerge from the model in a generic was, but is explicitly built in at the level of the individual oscillators and also reflected in the synaptic weights. Consequently, the model is very sensitive to variations in the frequencies of the oscillators: Unless all of the frequencies are varied in the same way, even small variations abolish the ability to discriminate between intervals. In consequence, the model only allows a certain type of noise, namely scalar variability in the phase of the spike times, a restriction that is unlikely to be fulfilled in the brain.

\subsection{Information Theory of Time}

From a mathematical point of view, the models of time perception we discussed in the previous section were based on deterministic differential and algebraic equations. Although some of them included terms of synaptic background activity or a distribution of parameters, the temporal processing is based on the deterministic dynamics, and the stochastic elements are either seen as a nuisance that impairs robust and precise time perception, or as way to generate heterogeneity of parameters in a network.

Recognizing the inherent presence of noise and variability both in the brain and in the external world, one could also take a different perspective and treat all dynamics in the brain as stochastic processes in the first place, and analyze their probability distribution functions (PDF). This leads to the well-established theory of stochastic inference, which is based on information theory. The deterministic picture can be retained by estimating the mean of the probability distribution. But the advantage of the stochastic approach is that the dynamics of higher moments, such as the variance and covariance, are more easily accessible. Furthermore, the framework of stochastic inference allows to formalize the concept of optimality in a very straightforward way, a concept that is of central importance for the present investigation.

\subsubsection{Stochastic inference}

Here, we review some basic concepts and definitions of stochastic inference which are relevant to our studie $\sqrt{15}$. A stochastic process is defined as a family of stochastic variables $X_{t}$ in a common probability space $\{\Omega, \mathcal{F}, P\}$ which are parameterized by a time index 16 . The condition of a common probability space implies that the variables are distributed by a joint probability distribution $P\left(X_{t}\right)$ which may depend on time itself. Now, assume that a set of data $x_{t}$ is given that can be seen as a specific realization of such a stochastic process. In such a situation, stochastic inference is used to gather information about the probability distribution of this stochastic process from the data. In the most extreme case, there is no a priori knowledge about $P\left(X_{t}\right)$, so the entire probability distribution must be estimated from

\footnotetext{
${ }^{14}$ The dopaminergic modulation of the clock speed could not be explained, however.

${ }^{15}$ These fundamentals can be found in standard textbooks of information theory, e.g. 92

${ }^{16} t$ can be both discrete or continuous
} 
the data. We restrict ourselves to the somewhat easier case of parametric estimation, where the functional form $\left(P\left(X_{t} \mid \theta\right)\right)$ of the PDF is known, but depends on some unknown parameters $\theta$ which must be estimated. When the probability distribution is regarded as a function of these parameters, and the values of the random variables are given by the data, it is also called the likelihood to obtain the data $x_{t}$, given the parameter value $\theta: L(\theta)=P\left(x_{t} \mid \theta\right)$. The Gaussian distribution is a prominent example of a case where only two parameters, mean and variance, determine the distribution, and thus the stochastic process.

To approximate the true value of such a parameter, one constructs an additional stochastic variable which is a function of $X_{t}$, a so-called estimator. Using the actual realizations of $X_{t}$ that is given by the data, one can compute an estimate of the actual parameter from this function. An example is the arithmetic mean over a data set, which is an estimator for the true expectation value of the underlying distribution. As for any other stochastic variable, there is a probability distribution assigned to an estimator which allows to define measures of its quality. First, the distribution of an estimator should be centered on the true value of the parameter. According to this demand, an estimator $\hat{\theta}$ of a parameter $\theta$ is called unbiased if and only if $B(\hat{\theta})=E(\hat{\theta}-\theta)=0 . B(\theta)$ is called the bias of the estimator. Similarly, is is desirable that the distribution of $\hat{\theta}$ has a small variance, such that any representative data set yields similar estimates. It can be shown that the lowest possible variance of an unbiased estimator is given by the Cramer-Rao bound, which is the inverse of the Fisher information

$$
\operatorname{Var}(\hat{\theta}) \geq \frac{1}{I_{F}(\theta)}
$$

The Fisher information is the expectation value of the squared derivative of the log-likelihood function

$$
I_{F}(\theta)=E\left[\left(\frac{\partial}{\partial \theta} \ln L(\theta)\right)^{2}\right],
$$

where $E\{X\}$ is the expectation value of $X$. An estimator is called efficient if its variance is a low as theoretically possible, that is, equal to the Cramer-Rao bound.

To make more sense out of the concept of Fisher information, we consider another quantity derived from $L(\theta)$, the score $V=\partial_{\theta} \ln L(\theta)$. It can be shown that the mean of the score is zero. Thus, this random variable is expected at the value of $\theta$ where the likelihood function has an extremum. This is interesting in the context of constructing an estimator for the parameter: Using a value of $\theta$ where the likelihood is maximal appears to be a good estimation of the real value of $\theta$. Indeed, this maximum likelihood estimator

$$
\hat{\theta}_{\mathrm{ML}}=\operatorname{argmax}_{\theta}\{L(\theta)\}
$$

can be shown to be both unbiased and efficient in the limit of a large number of observations (also called asymptotically unbiased and efficient). Now, it can be seen that the Fisher information is the variance of the score at the point of the maximum-likelihood $\theta_{\mathrm{ML}}$. Furthermore, the Fisher information can also be written as

$$
I_{F}(\theta)=-E\left[\frac{\partial^{2}}{\partial \theta^{2}} \ln L(\theta)\right]
$$


if certain general conditions apply. In this form, the Fisher information measures the (expected) sharpness of the likelihood function close to its maximum: A sharper likelihood curve near the maximum implies that the maximum is more clearly separated from its environment.

In its definition, the likelihood function is technically written as a conditional probability of the observed data, given a certain parameter value $\theta\left(L(\theta)=P\left(x_{t} \mid \theta\right)\right)$. It is possible to rewrite the likelihood function as a probability distribution of $\theta$, given the data $\left(P\left(\theta \mid x_{t}\right)\right)$, using Bayes' rule

$$
P\left(\theta \mid x_{t}\right)=\frac{\left.P\left(x_{t} \mid \theta\right)\right) P(\theta)}{P\left(x_{t}\right)}=\frac{L(\theta) P(\theta)}{P\left(x_{t}\right)} \propto L(\theta) P(\theta) .
$$

$P\left(x_{t}\right)$ does not depend on the parameter $\theta$ and can be considered as a constant normalization factor. $P(\theta)$ is called the prior distribution of the parameter. Thus, using $P\left(\theta \mid x_{t}\right)$ as the basis of parameter estimation allows to formalize a priori knowledge about the parameter, and to use it in order to improve the estimate. The analogue to the maximum-likelihood estimator using $P\left(\theta \mid x_{t}\right)$ is the maximum a posteriori estimator

$$
\hat{\theta}_{\mathrm{MAP}}=\operatorname{argmax}_{\theta}\left\{P\left(\theta \mid x_{t}\right)\right\} .
$$

Examples of prior knowledge about $\theta$ include an eligible range of parameter values (reflected in a rectangular-shaped prior distribution), or an certain value that is expected due to previous experience (which may modeled by a Gaussian distribution centered on the expected value, with a variance reflecting the uncertainty of the prior estimate). This approach is also applied to estimation problems in the brain, where the prior reflects top-down information such as memory or expectation which modulates sensory information.

\subsubsection{Fisher information and time estimation}

The framework of stochastic inference can also be applied to time perception. In this perspective, time-dependent processes such as spatio-temporal spike patterns or firing rates are seen as stochastic processes. The time interval between two stimuli that influence the state of the brain can then be estimated from observing the realizations of these processes at the two points in time. One advantage of this approach is that the mean and the variance of a duration estimate directly correspond to the PSE and the DL of the psychophysical experiments, and that the mathematical framework of estimation theory can thus be used to predict the outcome of such experiments, given that the probability distribution underlying the stochastic processes in the brain follows the assumed form. Conversely, the psychophysical results can also be used to constrain the probability distribution, and thus the classes of models that can be used for time perception.

Ahrens and Sahani [5] were the first to use this approach for a simple Gaussian process $y(t)$ with an exponentially decaying covariance as the only time-dependent process. They showed that even such a simple process that does not change with time in the mean, can still be used for time perception, based on the noise correlations only. To estimate an interval $\Delta t$, they used the observations of the process at the two points in time $t$ and $t+\Delta t$, and the likelihood 
$L(\Delta t)=P(\{y(t), y(t+\Delta t\} \mid \Delta t)$ to estimate $\Delta t$. As the process is stationary (the covariance only depends on $\Delta t$ ), this estimate is the same for all values of $t$. Ahrens and Sahani calculated the Fisher information with respect to $\Delta t$ from this process, and concluded that the lower bound for the estimation error obeys Weber's law when multiple processes with different time scales are used simultaneously for estimation. In Chapter 4 , we extend this framework to more complex Gaussian processes which may contain temporal information in the mean, the variance and the covariance. We study the relative contributions from each of these moments, and conclude that Weber's law only holds exactly if only the (linearly increasing) variance of a process is used for the estimation of time, in contrast to the claims of the previous study.

\subsubsection{Baysian integration of temporal multi-sensory information}

The Bayesian framework also provides a formal optimization criterion for the integration of multi-sensory integration: If there is information about a perceptual quantity, such as the position of an object in space or the duration of a stimulus which comes from different sensory modalities, how should this information be integrated to yield the optimal estimate about that quantity? This question is of particular importance in situations where the reliability of perception varies between modalities. For instance, it is much easier to judge the position of a light compared to the position of a tone [34, whereas duration estimates are more reliable in the auditory compared to the visual domain (cf. Section 2.1.2.3). Intuitively, one would say that the estimates from the two modalities should be weighted with their respective reliability to result in an optimal total estimate. Indeed, estimation theory allows for a formalization of this intuition. If there is sensory input $r_{A}$ from modality $A$ and $r_{B}$ from modality $B$, and the likelihood functions for an estimated parameter $\theta$ (such as position or interval duration) based on the two modalities are given by $L_{A}(\theta)=P\left(r_{A} \mid \theta\right)$ and $L_{B}(\theta)=P\left(r_{B} \mid \theta\right)$, respectively, it can be shown that the information content of a combined likelihood $L_{A B}(\theta)$ is maximal when the two individual likelihoods are multiplied, $L_{A B}(\theta)=L_{A}(\theta) L_{B}(\theta)$. Furthermore, an optimal estimate $\hat{\theta}_{A B}$ from both modalities is given by a linear combination of the estimates $\hat{\theta}_{A}$ and $\hat{\theta}_{B}$ from the individual modalities [152]

$$
\hat{\theta}_{A B}=\frac{I_{F}^{A}(\theta) \hat{\theta}_{A}+I_{F}^{B}(\theta) \hat{\theta}_{B}}{I_{F}^{A}(\theta)+I_{F}^{B}(\theta)} .
$$

$I_{F}^{A}(\theta)$ and $I_{F}^{B}(\theta)$ is the Fisher information about $\theta$ which is computed from the likelihood of modality $A$ and $B$, respectively. This complies with the intitive picture given above: The individual estimates are weighted with the relative information from each modality. If the likelihood takes the form of a Gaussian distribution for both modalities, the Fisher information is given by $I_{F}^{A}(\theta)=1 / \sigma_{A}^{2}$ and $I_{F}^{B}(\theta)=1 / \sigma_{B}^{2}$, where $\sigma_{A}^{2}$ and $\sigma_{B}^{2}$ are the variances of the estimate from modality $A$ and $B$, respectively. Using a maximum-likelihood estimator, the estimates from the individual modalities are equal to the respective means of the two likelihoods, $\bar{\theta}_{A}$ and $\bar{\theta}_{B}$, and the common estimate simplifies to 34]

$$
\hat{\theta}_{A B}=\frac{\bar{\theta}_{A} / \sigma_{A}^{2}+\bar{\theta}_{A} / \sigma_{B}^{2}}{1 / \sigma_{A}^{2}+1 / \sigma_{B}^{2}} .
$$

The concept of Bayesian multi-sensory integration has been shown to hold for several tasks and modalities [34, 101]. For instance, when a visual and an auditory cue are considered to 
originate from a common source in space, information from both modalities are combined to localize this source. Alais and Burr [6] tested whether this integration is optimal in the Bayesian sense by measuring the distribution of the localization estimate based on the visual or the auditory information alone, and on both cues combined. They also manipulated the reliability of the visual source by blurring it in a systematic way. For each level of visual reliability, Eq. 2.24 was found to hold. Other examples include the localization of one's hand based on a combination of visual and proprioceptive cues, and the inference of depth information from visual and haptic information [34].

However, Bayesian integration has not yet been directly tested for the multi-sensory integration of temporal information. In the only study were are aware of [174], the authors made participants discriminate between two intervals presented in different (visual and auditory) modalities, and compared both DL and PSE with experiments where both intervals were in the same modality. They also compared the results of their experiment with a variant of the PAM model (cf. Section 2.1.3.2), which allowed the combination of probability distributions from individual modalities. Regarding subjective duration, the experiments tended to confirm the predictions of the model. However, this experiment is different from the scenario where two pieces of sensory information are presented at the same time. In their model, the mean of the combined probability distribution does not depend on the variance of the individual probability distributions, but only on its mean.

In Chapter 5, we investigate direct cross-modal interaction of time perception between the visual and the auditory modality, and between time perception and motor timing, using a distortion approach. 


\section{Time perception by optimal synaptic selection of synfire chains}

As mentioned in the introduction of the thesis, the theoretical investigations of time perception are often quite separated in the fields of psychology and neuroscience, in the sense that psychophysical theories most often lack a discussion about their neural basis, and most neurocomputational models do not make predictions about psychophysical experiments. However, combining the predictive power of both approaches may lead to progress in both fields at the same time: Psychophysical experiments may be used to constrain the choice of a neurocomputational model of time perception, and such a model may in turn provide a generic explanation for psychophysical phenomena that would require ad hoc assumptions otherwise.

In this chapter, we present an example for such a joint modeling approach. We show that a neurocomputational model based on a set of synfire chains (cf. Section 2.2.2) with different speeds of transmission can account for the U-shaped form of the Weber fraction that was observed in several experiments (Section 2.1.2.2). To see how this comes about, we first show that an increase in the transmission time corresponds to a superlinear increase of the timing errors. The experimentally observed error function then emerges from optimal selection of chains for each given interval, if the length of the chain is assumed to be finite. Furthermore, we show how this optimal selection could be implemented by competitive spike-timing dependent plasticity in the connections from the chains to a readout network, and discuss implications of our model on selective temporal learning and possible neural architectures of interval timing. This model constitutes our first example of how constrained optimization in neural processes leads to psychophysical results. A slightly modified version of this chapter has been published in the Journal of Computational Neuroscience in 2008 [66].

\subsection{Introduction}

Our world changes in time, and our brain faces the challenge to cope with these changes. Sequences of stimuli often convey information in their order and timing, e.g. in speech or music. Our sense of causality requires knowledge about the natural temporal order in which events happen. Our brain can use this knowledge together with information about the typical duration of events to predict the evolution of sequences of events. Also on the level of behavior, timing is obviously crucial, as a given action can be right or wrong based only on the time of its execution.

Recognizing this importance, many researchers have posed the question of how time is represented in the brain. For some time-related stimulus features such as the speed of motion in the visual field or the frequency of a tone, such representations could be found in distinct brain areas such as the middle temporal region (MT) of visual cortex for speed and the inner hair 
cells for tone frequency. For the duration of a stimulus or the interstimulus interval, however, no single neural correlate has been identified [28]. Instead, lesion and imaging studies have revealed the possible participation of a whole network of structures such as the cerebellum, the basal ganglia, the thalamus and various cortical regions such as prefrontal cortex (PFC) and the supplementary motor area (SMA) 28, 110, 81]. This spectrum of brain structures is accompanied by a variety of possible timing mechanisms. Basically, any process in the brain could be used to represent the time that has elapsed while the process unfolds. Models of temporal processing have exploited neural structures that range from single neurons [168, 62, neural oscillators read out by coincidence detectors [119] and short-term synaptic plasticity [25] to reverberating loops within the cerebellum [131, 188], slowly climbing activity in PFC neurons during working memory tasks [40] and stochastic decay of memory traces [98].

These neurobiological models focus on the ability of a given neuronal circuit to represent temporal information. However, most of them are not sufficient to explain behavioral performance in timing tasks, as they do not discuss errors in the representation. Temporal precision and the change of timing performance under various conditions are one major subject of psychological experiments in both animal and man [61, 58]. Results from such experiments can constrain neuronal models regarding their predictions on timing errors. A typical class of experiments is given by the task of interval reproduction [9]. The participants are presented with an interval of duration $T$, e.g. a continuous tone or a series of flashes of light. Afterwards, they are asked to reproduce this interval for example by pressing a button as long as they think the interval was. This experiment results in a set of reproduced intervals, usually clustered around some mean $m$ with a standard deviation $\sigma$. These two measures are used to characterize the participant's ability to reproduce the interval $T$. To explain the results from such experiments, information-processing models are used. They are composed from a set of functional processing stages which interact with each other and provide some understanding of the mechanisms behind the experimental results. The most popular of these models is the pacemaker-accumulator system [33]: An oscillating or random process (the pacemaker) generates pulses with a fixed frequency, while another system (the accumulator) counts the number of these pulses. The number of pulses accumulated during an interval is used to estimate the duration of thet interval. This theory has been formalized in several ways [55, 95] and can explain a wide range of phenomena [61, 58]. Another widely used concept is called interval timers [77] or labeled lines [28]. Models within this framework assume a number of units which operate with different time constants, such that each of these units is tuned to a specific interval of time.

While information-processing models are designed to explain timing errors in behavioural experiments, they are only rarely connected to some neural substrate and thus can not identify the source of these errors. To connect the experimental results with a proposed timing mechanism in the brain, it is useful to assume that the brain performs an estimation of the time elapsed since the press of a button, such that the participant releases the button when the memorized duration of the target interval is reached. The estimation will be subject to timing errors $\sigma$ and possibly also some bias $m-T$ if the participant responds systematically too early or too late. Taking this view, a neuronal model of temporal processing is supported 
by psychophysical experiments if its estimation statistics are compatible with the response statistics found in the experiment.

One prominent finding of timing experiments is that timing errors increase monotonically with the duration of the interval to be processed. This increase is characterized by the Weber fraction, defined by $\sigma / m$. The shape of this fraction as a function of the interval $T$ is then to be reproduced by a neuronal model. According to "scalar expectancy theory" (SET, [55]), temporal perception obeys Weber's law, which means that the Weber fraction does not depend on $T$. Indeed, many studies were conducted that seem to confirm this "scalar property" [58]. However, a model that tries to explain a constant Weber fraction faces an inherent problem: If the timing errors arise from noise affecting the timing units without correlations, or with a finite correlation length, these timing errors will increase as $\sqrt{T}$, as the variances $\sigma^{2}$ add up linearly over time. Thus, the observed linear increase of the timing error needs another error source. SET, and also some other information-processing theories solve this problem by assuming that the observed scaling of the errors is built in one of the processing stages such as the counter [95] or a memory stage [55]. Some of the neuronal models also use ad hoc assumptions about scalar variability, e.g. in the distribution of synaptic weights [119] or in the rise times of firing rates [62], to generate the scalar property. Others assume special properties of the noise, such as a low-pass filtered frequency band [168] or independence of the stimulus duration [162]. There are also a few attempts to explain Weber's law by inherent properties of the model [140]. However, none of these studies discusses the relation of the steeply increasing errors with the principle of optimality: If it is possible to represent time with an error of the order of the square root of the interval duration, why should a brain under evolutionary pressure use some mechanism that is worse than that? Moreover, there is also evidence that the scalar property is not universally valid in temporal processing. The Weber fraction increases both at short and long intervals, with a minimum in between [15, 39, 54]. While the increase at short intervals can be explained by an additional, time-independent error ("generalized Weber's law"), the increase at longer intervals was mostly ignored in information-processing models (but see [162]), because it is not compatible with the predictions from SET. To date, there is no neurocomputational model that accounts for the entire Weber fraction with its decreasing, constant, and also increasing parts.

In this chapter, we offer a model in which the U-shaped form of the Weber function emerges directly as a result of an optimization process. The model consists of a number of timing units with different time constants. These units project onto a set of readout neurons, which show a unique spike pattern for each interval to be represented. In this framework, synaptic plasticity implements an optimal selection of timing units under limited resources. For the units itself, we demand high temporal precision and reliability to obtain optimal results, and also a sufficiently wide range of time constants. In a noisy brain, arguably the most precise temporal code is provided by synfire chains [2]. A synfire chain is a feed-forward network with strongly converging and diverging connectivity. Such a network is able to stably propagate a wave of neural activity from pool to pool at a precision in the range of milliseconds [70, 37] even in the presence of usual biological inaccuracies. This property makes synfire chains exquisite timing devices: If an activity volley is injected at stimulus onset, the time elapsed after the 
onset is reliably converted into the position of the volley in the chain. In this way, synfire chains can integrate two of the main concepts of timing: If each of the pools is used, the chain is equivalent to a delay line and can be compared to a pacemaker-accumulator system, in the sense that each pool corresponds to a pulse that is accumulated and the transmission speed from one pool to the next corresponds to the pulse frequency. On the other hand, it is also possible to use several chains as interval timers, if they have different transmission speeds and only the last pool of each chain is used for time estimation. And finally, it is also possible to connect the final pool of a chain with the first one, which results in a neural oscillator with a frequency dependent on the number of pools and the transmission speed.

For a single synfire chain, timing errors again increase like the square root of the interval length. However, we arrive at the result that the timing errors increase super-linearly with the delay of transmission from one pool to next. As the number of pools in a chain can be assumed to be limited, this constitutes an additional error source for longer intervals, as they can only be represented by chains with a larger delay. Under these conditions, we show that the observed U-shaped form of the Weber fraction arises from optimal selection of the chain with the lowest possible timing error for any given interval to be represented. Finally, we propose a combination of spike-timing dependent plasticity (STDP [13]) and homeostatic plasticity [172] for the connections from the chains to the readout neurons which implements an optimal and unique selection of chains. This selection is based on the fact that the effective learning rate of STDP depends on the temporal distribution of the input pattern.

The chapter is organized as follows: In Section 3.2, we describe the model structure and provide the equations that are used to simulate the neurons, synapses and plasticity rules. Section 3.3 .2 defines the notions of transmission delay and timing error and demonstrates how the temporal statistics of synfire chains affect temporal processing. Furthermore, we discuss the effect of variations in the model parameters, especially the rise time of the postsynaptic potentials (PSPs) on the delay and the timing error. In Section 3.4, we combine these results to a theory of optimal temporal processing and show how the U-shaped form of the Weber fraction emerges. Section 3.5 discusses the implementation of the optimal and unique selection of chains by synaptic plasticity. Finally, in Section 3.6 the results are discussed regarding their implications for selective learning, effects of attention, and also possible extensions of the model.

\subsection{Neuron and network model}

\subsubsection{Network structure}

The model consists of neurons which are described by their membrane potential $V_{i}$, and connected by synapses of strength $w_{i j}$, where $i$ denotes the postsynaptic and $j$ the presynaptic neuron. The neurons are organized in different networks (see Fig. 3.1): Synfire chains consist of $L$ pools denoted by $\mathcal{P}_{l}$ which contain $N$ neurons each. Each neuron in a pool $\mathcal{P}_{l}$ has a 

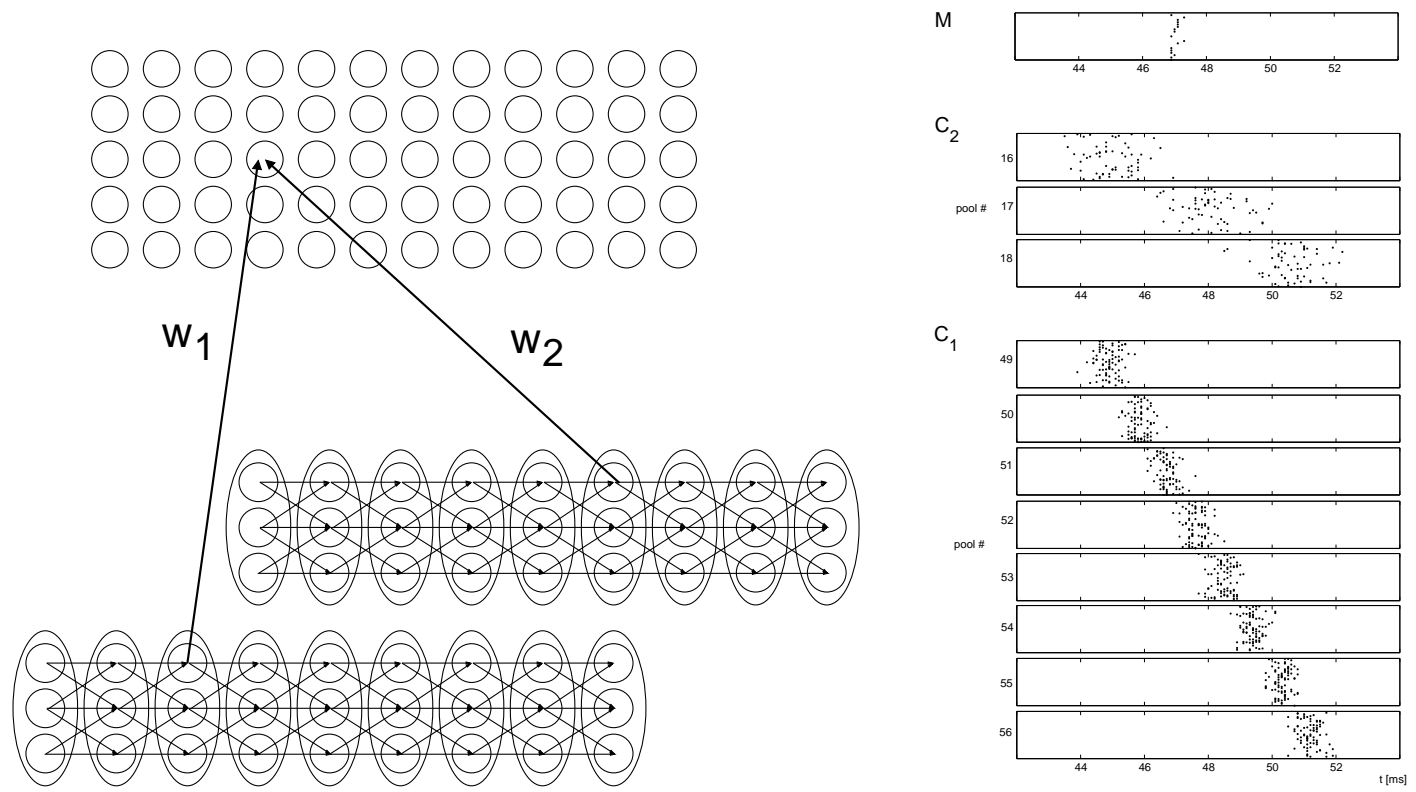

Figure 3.1.: Left: Illustration of the model structure. A readout network $\mathcal{M}$ receives convergent connections to from different synfire chains such as $\mathcal{C}_{1}$ and $\mathcal{C}_{2}$. By the competition between the respective weights, $w_{1}$ and $w_{2}$, the network determines which chain is the optimally responds at a time interval represented by the output unit in $\mathcal{M}$. Right: Raster plot showing the spikes in the readout network $\mathcal{M}$ and selected pools from the chains $\mathcal{C}_{1}$ and $\mathcal{C}_{2}$. Each dot corresponds to a spike. In $\mathcal{C}_{1}$, activity propagates faster and with smaller jitter $\sigma_{P}$ compared to $\mathcal{C}_{2}$.

probability of $p_{S}$ to be connected to any neuron in the subsequent pool $P_{l+1}$ with strength $w_{S}$

$$
p\left(w_{i j}\right)=\left\{\begin{array}{rl}
p_{S} & \text { for } w_{i j}=w_{S} \\
1-p_{S} & \text { for } w_{i j}=0
\end{array} \quad \forall i \in \mathcal{P}_{l+1}, j \in \mathcal{P}_{l} .\right.
$$

If all neurons in pool $\mathcal{P}_{l}$ fire nearly synchronously with a small temporal jitter, this induces on average $N w_{S}$ inputs in each neuron in the subsequent pool $\mathcal{P}_{l+1}$. Thus, the firing times from the preceding pool are averaged and the jitter is reduced in the firing times of pool $\mathcal{P}_{l+1}$. As each neuron in pool $\mathcal{P}_{l+1}$ in turn projects on average to $N w_{S}$ neurons, the activity in pool $\mathcal{P}_{l+2}$ will be even more synchronized. If all neurons in the chain are disturbed by synaptic noise, the temporal jitter will not decrease to zero, but converge to a near-synchronized fixed point where the effect of the connectivity and the noise are balanced [70, 37].

Apart from the synfire chains, there is a readout network $\mathcal{M}$ consisting of $M$ neurons with no connections among each other $\left(w_{i j}=0 \quad \forall i, j \in \mathcal{M}\right)$, but which connections from the synfire chains. A pool $P_{l}$ is connected to a readout neuron $i \in M$ by the rule

$$
p\left(w_{i j}\right)=\left\{\begin{array}{rl}
p_{M} & \text { for } w_{i j}=w_{S} \\
1-p_{M} & \text { for } w_{i j}=0
\end{array} \quad \forall j \in \mathcal{P}_{l} .\right.
$$




\begin{tabular}{|c|c|c|c|c|}
\hline \multicolumn{2}{|c|}{ network parameters } & \multicolumn{2}{|c|}{ neuron and synapses } & synaptic plasticity \\
\hline$L$ & 120 & $V_{\text {rest }}$ & $-60 \mathrm{mV}$ & 0.2 \\
\hline$N$ & 100 & $\tau$ & $30 \mathrm{mV}$ & $A_{d} \quad 0.21$ \\
\hline$M$ & 20 & $V_{\text {thr }}$ & $-40 \mathrm{mV}$ & $\tau_{p} \quad 5 \mathrm{~ms}$ \\
\hline$p_{S}$ & 0.345 & $V_{\text {reset }}$ & $-65 \mathrm{mV}$ & $5 \mathrm{~ms}$ \\
\hline$w_{S}$ & 0.375 & $\lambda_{+}$ & 96.9 & $g_{P}$ \\
\hline & 0.1 & $\lambda_{-}$ & 92.29 & $g_{I} \quad 0.015$ \\
\hline$w_{M}$ & 0.3 & $\epsilon_{+}$ & 0.1 & \\
\hline & & & 0.1 & \\
\hline & & $\alpha$ & $0.5 \mathrm{~ms}$ & \\
\hline
\end{tabular}

Table 3.1.: Values of all model parameters that are used unless otherwise stated.

The set of all neurons in a given synfire chain is denoted by $\mathcal{C}_{\alpha}$, as all the parameters are identical across chains except for $\alpha$, which is defined below. The values of all parameters regarding network connectivity are listed in the left column of Table. 3.1.

\subsubsection{Neuron model and synapses}

The neurons are modeled as leaky integrate-and-fire units embedded in a stochastic background network. While the membrane potential $V_{i}$ stays below a threshold $V_{\text {thr }}$, the time evolution of $V_{i}$ is given by

$$
\tau \frac{d V_{i}}{d t}=-\left(V_{i}-V_{\text {rest }}\right)+\sum_{k: w_{i k} \neq 0} \frac{w_{i k}}{\alpha} \sum_{j=1}^{S_{k}} \operatorname{PSP}\left(t-t_{k j}^{\mathrm{sp}}\right)+I_{\text {noise }}+I_{\text {ext }} .
$$

The first term models the leakiness of the neuron, while the others describe its input. Without any input $\left(I_{\text {noise }}=I_{\text {ext }}=0, S_{k}=0 \forall k\right), V_{i}$ relaxes exponentially to the resting potential $V_{\text {rest }}$ with time constant $\tau$. The second term is a sum over all $S_{k}$ spikes in all neurons $k$ which the neuron is connected to. $t_{k j}^{\mathrm{sp}}$ denote the times of each of these spikes, where $j$ the number of the spike. $I_{\text {noise }}$ represents synaptic input from the stochastic background network, which is collapsed into an excitatory and an inhibitory part

$$
I_{\text {noise }}=\epsilon_{+} \mathcal{N}\left(\lambda_{+}, \lambda_{+}\right)-\epsilon_{-} \mathcal{N}\left(\lambda_{-}, \lambda_{+}\right),
$$

where $\epsilon_{+}, \epsilon_{-}>0$ and $\mathcal{N}\left(m, \sigma^{2}\right)$ denotes a random variable with a Gaussian distribution with mean $m$ and standard deviation $\sigma$. In this form, the Gaussians approximate two Poisson processes with rates $\lambda_{+}$and $\lambda_{-}$, respectively. Using the standard parameter values (see Table 3.1, middle column), ignoring the threshold $V_{\mathrm{thr}}$ and without further input, the membrane potential converges to a mean of $\langle V\rangle=-46.4 \mathrm{mV}$ and a standard deviation of $\sigma_{V}=1.4 \mathrm{mV}$.

Whenever the membrane potential $V_{i}$ crosses the threshold $V_{\text {thr }}$ from below, the neuron $i$ fires a spike. $V_{i}$ is then set to the reset potential $V_{\text {reset }}$ and the current time $t$ is included in the set of firing times of neuron $i$ by increasing the number of spikes $S_{i}$ by one $\left(S_{i} \rightarrow S_{i}+1\right)$ 
and setting $t_{i S_{i}}^{\mathrm{sp}}=t$. A spike in neuron $j$ influences the membrane potential $V_{i}$ of all neurons $i: w_{i j} \neq 0$ it is connected to presynaptically. The time evolution of the induced PSP in neuron $i$ is described by an $\alpha$ function

$$
\operatorname{PSP}(t)=\frac{t}{\alpha} \exp \left(-\frac{t}{\alpha}\right),
$$

where $\alpha$ is the rise time of the PSP. The synaptic weights $w_{i j}$ in Eq. 3.3 are normalized by $\alpha$ to ensure that the total impact of a single spike on the postsynaptic membrane potential does not change with $\alpha$. As mentioned before, different synfire chains denoted by $\mathcal{C}_{\alpha}$ will differ only in this parameter. No additional synaptic delays are incorporated, so $\alpha$ is the only parameter that determines the time course of the PSP. Introducing a distribution of delays does not qualitatively change the results.

\subsubsection{Synaptic plasticity}

The connections from the chains to the readout neurons $\left\{w_{i j}: i \in \mathcal{M}\right\}$ are subject to two forms of synaptic plasticity: STDP [13] and homeostatic plasticity 172]. STDP is applied after each spike $t_{i k}^{\mathrm{sp}}$ in neuron $i$. For all spikes $t_{j l}^{\mathrm{sp}}$ in neurons that are connected to neuron $i$ $\left\{t_{j l}^{\mathrm{sp}}: w_{i j} \neq 0 \vee w_{j i} \neq 0\right\}$, the time $t=t_{j k}^{\mathrm{sp}}-t_{i l}^{\mathrm{sp}}$ is calculated and the respective weights are updated by adding

$$
\Delta w=\left\{\begin{aligned}
A_{p} \exp \left(-t / \tau_{p}\right) & \text { if } t>0 \\
-A_{d} \exp \left(-t / \tau_{d}\right) & \text { if } t<0
\end{aligned}\right.
$$

Thus, the synapse between two neurons is strengthened if the presynaptic spike occurs earlier than the postsynaptic spike $(t>0)$, but if this order is reversed $(t<0)$, the synapse is weakened. This introduces the notion of causality into the learning rule.

Synaptic weights under control of the STDP learning rule either decays to zero or diverges, depending on the network's activity [175]. One way to prevent this is to complement STDP by a homeostatic learning rule, which adjusts the synaptic weights such that the network achieves a certain mean firing rate $a_{\text {goal }}$. A simple model of this mechanism is given by a proportional-integral feedback controller [175]

$$
\frac{d w}{d t}=g_{P} w\left(a_{\text {goal }}-a\right)+g_{I} w \int_{0}^{t} d t\left(a_{\text {goal }}-a\right)
$$

This equation is applied to update $w$ after each trial based on the actual mean firing rate $a$ of all readout neurons. This seems more appropriate then using it every time step, as homeostatic plasticity is believed to act slowly [172]. Parameter value for both forms of plasticity are listed in Table 3.1, right column.

\subsection{Temporal statistics of synfire chains}

\subsubsection{Quasi-spatial representation of time}

An interval can be represented by a synfire chain if the stimulus onset triggers a volley of activity in the first pool $\mathcal{P}_{1}$ of the chain. This volley can be characterized by the number of 
spikes $a(1)$ in the first pool and the temporal jitter $\sigma_{P}(1)$ of these spikes around the mean $t(1)$, which is defined to be zero. We assume that the volley follows a Gaussian distribution $\mathcal{N}\left(t(i), \sigma_{P}(i)^{2}\right)$, which turns out to be a good approximation for the simulated data. For a large range of initial conditions $\left(a(1), \sigma_{P}(1)\right)$, activity will then stably propagate through the pools, approaching a fixed point $\left(a, \sigma_{P}\right)$ in both parameters [37]. The time at which the activity wave has reached pool $\mathcal{P}_{i}$ can be estimated by the mean spike times of the neurons in that pool

$$
t(i)=\frac{1}{a(i)} \sum_{n=1}^{N} \sum_{j=1}^{S_{n}} t_{n j}^{\mathrm{sp}}, \quad \text { with } a(i)=\left|\left\{t_{n j}^{\mathrm{sp}}: n \in \mathcal{P}_{i}\right\}\right| .
$$

$a(i)$ is the number of spikes in pool $\mathcal{P} i$, which may also be larger than $N$ if there are neurons that spike more than once. As we only look at the firing statistics of neurons averaged over pools, $S_{n}$ denotes the number of spikes in the pool $n$, rather than in an individual neuron. For practical calculation in simulations, only those spikes enter this equation which give rise to a firing rate above a threshold value defined from the background firing rate. The rate is calculated by counting the number of spikes in a time bin of $0.1 \mathrm{~ms}$.

From $t(i)$, we define the transmission delay $\Delta t(i)$ from pool $\mathcal{P}_{i}$ to pool $\mathcal{P}_{i+1}$ as

$$
\Delta t(i)=t(i+1)-t(i) .
$$

An interval can only be reliably estimated if $\Delta t(i)$ has a robust relation to $i$. For a synfire chain, one would expect that $\Delta t(i)$ converges to a fixed point value $\Delta t$, as $a(i)$ and $\sigma_{P}(i)$ do. In Fig. 3.2 (left panel), $\Delta t(i)$ is shown for initial conditions $\left(a(1), \sigma_{P}(1)\right)=(100,4 \mathrm{~ms})$, which is close to the border of the basin of attraction of the fixed point $\left(a, \sigma_{P}\right)$. As expected, the mean of $\Delta t(i)$ converges to a fixed point after a short transient, establishing an approximately linear transformation of time into the position of the volley in the chain. This representation does not need to be literally spatial, as the pools are defined only by the topology of their connections and not their spatial position.

The exact form of the transient depends on the initial conditions. For instance, starting close to the border of the basin of attraction that surrounds the fixed point results in an overshoot of $\Delta t(i)$ in the first pools (Fig. 3.2, left). In the following, we suppress the effect of the transients by choosing initial conditions that are close to the fixed point.

\subsubsection{Timing errors}

While the mean of $\Delta t(i)$ converges to a constant $\Delta t$, fluctuations in the actual realizations of $\Delta t(i)$ remain. They can be considered Gaussian random variables

$$
\Delta t(i)=\mathcal{N}\left(\Delta t, \sigma_{\Delta t}^{2}\right)
$$

with mean and standard deviation independent of $i$. The temporal jitter $\sigma_{\Delta t}$ can be derived directly from the parameters $a$ and $\sigma_{P}$ for the steady state. Adding two random variables with a Gaussian distribution results in a Gaussian variable where mean and variance are the sum of those from the two original variables. $\Delta T$ is the sum of two random variables $t(i+1)$ 

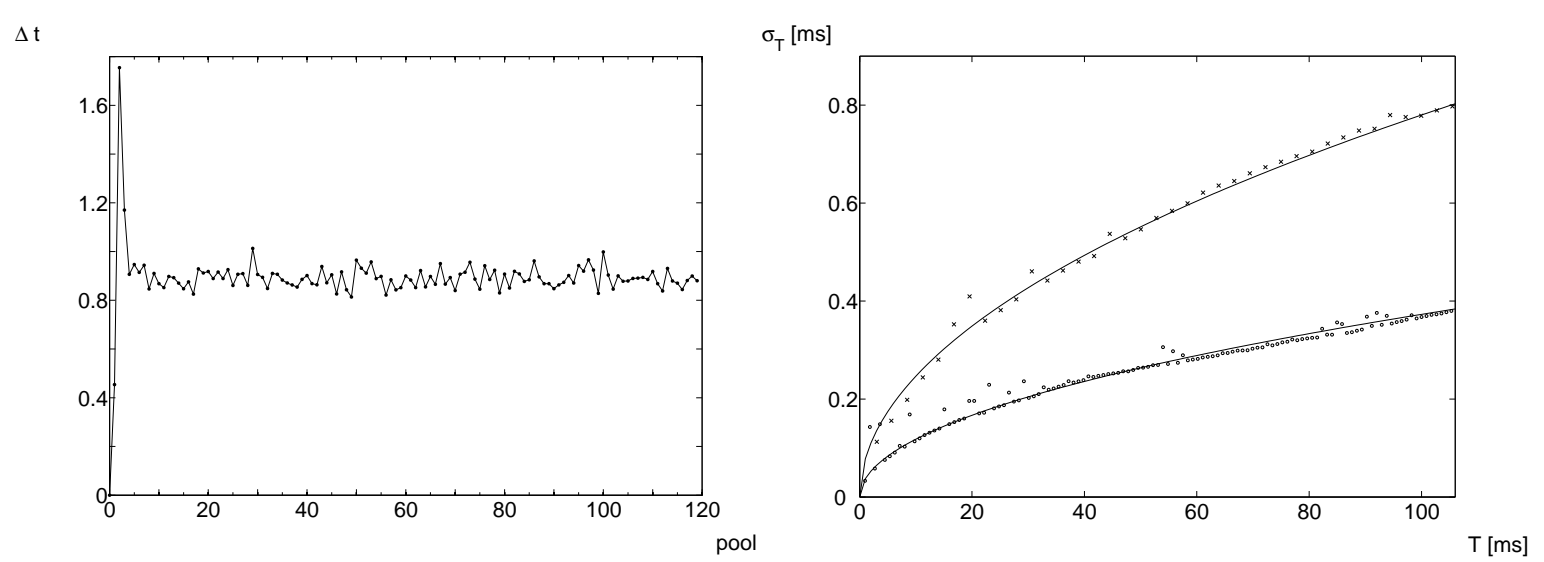

Figure 3.2.: Left: Transmission delay $\Delta t(i)$ as a function of the pool number $i$. After a transient, the mean of $\Delta t(i)$ converges to a constant $\Delta t$, while some finite jitter $\sigma_{\Delta t}$ remains. Right: Timing error $\sigma_{T}$ as a function of the stimulus duration $T$ for PSP rise times $\alpha=0.5 \mathrm{~ms}$ (lower curve) and $\alpha=1.5 \mathrm{~ms}$ (upper curve). The dots represent the simulation data and the line is a plot of Eq. 3.14 with $\sigma_{\Delta t}$ fitted to the data. The coefficient $\sigma_{\Delta t}$ is fitted to $0.035067( \pm 28)$ for $\alpha=0.5$ and 0.13093 $( \pm 15)$ for $\alpha=1.5$.

and $-t(i)$ which are themselves the sum of $a$ variables $t_{n j}^{\mathrm{sp}}$ each, divided by $a$. The spike times, in turn, have a Gaussian distribution with standard deviation $\sigma_{P}$ (cf. Sec. 3.3.1). As $a$ and $\sigma_{P}$ are constant in the steady state, both $t(i+1)$ and $-t(i)$ have a standard deviation of $\sigma_{P} / \sqrt{a}$, and $\sigma_{\Delta t}$, so that we find

$$
\sigma_{\Delta t}^{2}=\frac{2}{a} \sigma_{P}^{2}=\text { const. }
$$

The estimate of an interval $T$ can now be written as

$$
T(i)=\sum_{l=1}^{i} \Delta t(l)=\Delta t \cdot i+\mathcal{N}\left(0, \sigma_{T}^{2}\right)
$$

The second equality results from Eq. 3.10, $\sigma_{T}$ is the timing error which appears in psychophysical experiments. Again, the variances of $\Delta t(i)$ add up and the timing error results in

$$
\sigma_{T}(i)^{2}=\sum_{l=1}^{i} \sigma_{\Delta t}^{2}(l)=\sigma_{\Delta t}^{2} \cdot i,
$$

where the second equality holds at the fixed point value of $\sigma_{\Delta t}$ (Eq. 3.11). Using Eq. 3.12, one sees that the timing error increases with $\sqrt{T}$

$$
\sigma_{T}(T)=\sigma_{\Delta t} \cdot \sqrt{T}
$$




\begin{tabular}{|c|l|l|l|l|}
\hline parameter & $\mathrm{A}$ & $\mathrm{B}$ & $\mathrm{C}$ & explained variance \\
\hline$a$ & -1.298 & 1.21 & 98.20 & $83.8 \%$ \\
\hline$\Delta t$ & 0.1739 & 1.504 & 0.113 & $96.9 \%$ \\
\hline$\sigma_{P}$ & 0.01625 & 0.522 & 0.0348 & $92.5 \%$ \\
\hline \hline$\sigma_{\Delta t}$ & $2.677 \cdot 10^{-3}$ & $-8.833 \cdot 10^{-3}$ & 0.0486 & $82.4 \%$ \\
\hline
\end{tabular}

Table 3.2.: Coefficients of Eq. 3.15 fitted to the data sets of $a, \Delta t$ and $\sigma_{P}$ (first three rows), and coefficients of Eq. 3.16 fitted to the data set of $\sigma_{\Delta t}$ (final row).

Note that the timing error $\sigma_{T}$ and the spike time jitter $\sigma_{P}$ refer to two quite different concepts. $\sigma_{P}$ denotes the standard deviation of the spike times within a pool and also within the same trial. This jitter must not increase with time, or else activity can not be stably propagated. On the other hand, $\sigma_{T}$ is the accumulated error of the estimating the transmission delay $\Delta t$ across trials. Increase of $\sigma_{T}$ means that the temporal information encoded in the synfire chain is gradually lost at longer times, although it may still stably propagate activity in each individual trial.

In Fig. 3.2 (right panel), we have plotted the estimate of $\sigma_{T}$ for two different synfire chains $\mathcal{C}_{0.5}$ and $\mathcal{C}_{1.5}$, based on 1000 simulations each. One sees that both curves can be fitted with a $\sqrt{T}$-law, with $94.7 \%$ of the total variance explained for $\mathcal{C}_{0.5}$, and $95.2 \%$ for $\mathcal{C}_{1.5}$.

Linear representation of time and a $\sqrt{T}$ law for timing errors are also key properties of a pacemaker-accumulator system (cf. Section 2.1.3.2), so in this sense a synfire chain is equivalent to this class of models.

\subsubsection{Parameter variations}

A synfire chain can be seen as a timing device with a time constant of $L \Delta t$. In this section, we examine how we can increase $\Delta t$ to obtain different time scales without changing the pool number $L$.

\subsubsection{Effect of varying $\alpha$}

The position of the fixed point in $a$ and $\sigma_{P}$, and thus, $\Delta T$ and $\sigma_{\Delta T}$ depends strongly on the parameters of the synfire chain. The easiest way to change $\Delta T$ is to vary the rise time $\alpha$ of the postsynaptic potential. If the time until each spike fully affects the postsynaptic membrane potential increases, the transmission time of the entire volley will also be delayed. Moreover, this parameter has already been studied in its influence on the fixed point in $a$ and $\sigma_{P}$ [36].

We assess the effect of $\alpha$ by running simulations with 20 trials each, raising $\alpha$ by $0.05 \mathrm{~ms}$ after each set of trials. The dependency of $\Delta t, a$ and $\sigma_{P}$ on $\alpha$ turns out to be well fitted by a polynomial of second order

$$
f(x)=A x^{2}+B x+C .
$$



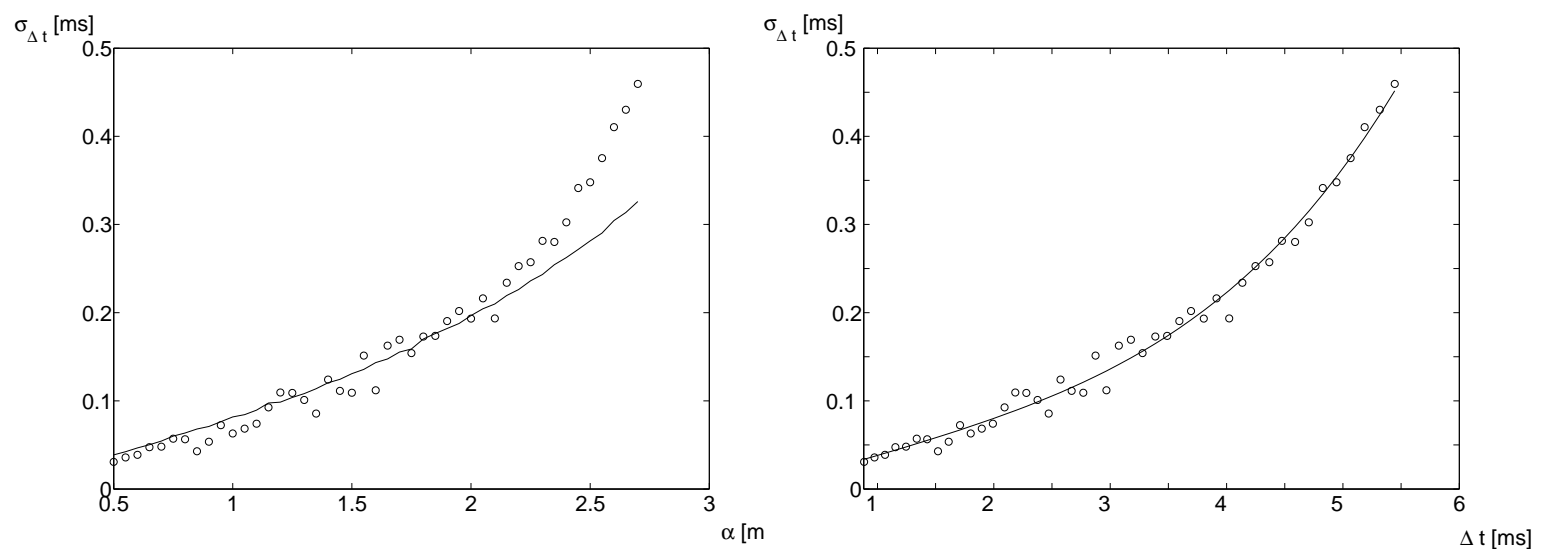

Figure 3.3.: Left: The variance in the transmission delay $\sigma_{\Delta t}$ as a function of the PSP rise time $\alpha$. Right: $\sigma_{\Delta t}$ as a function of mean transmission delay $\Delta t$. The dots are data from the simulation. In the left panel, the line is a plot of Eq. 3.11 with the values of $a$ and $\sigma_{P}$ from the simulation. In the right panel, the line is a fit to Eq. 3.16 using the parameters in Table 3.2, final row.

As seen from the coefficients in Table 3.2, $\Delta t$ and $\sigma_{P}$ increase mostly linear with $\alpha$. As $A \ll B$, the quadratic term only becomes relevant as a small correction at higher $\alpha$. $a$, on the other hand, decreases quadratically over the whole range of $\alpha$, but only moderately in total $(A, B \ll C)$. For $\sigma_{\Delta t}$, a fit to Eq. 3.15 only works well for small values of $\alpha$. Furthermore, when we check whether Eq. 3.11 holds for the simulated data, it turns out that it does only for $\alpha \leq 2$ (Fig. 3.3, left panel). As we are interested in the relationship between $\sigma_{\Delta t}$ and $\Delta t$, we fit a curve to the data points in these two dimensions. As a boundary condition, we demand $\sigma_{\Delta t}(0)=0$, as it makes no sense to assume a timing error if activity travels through the chain infinitely fast. With this constraint, the data turns out the be fitted very well with a third-order polynomial

$$
\sigma_{\Delta t}(\Delta t)=A \Delta t^{3}+B \Delta t^{2}+C \Delta t .
$$

The coefficients are listed in the final row of Table 3.2. Once again, the dependency is mostly linear, as $A, B \ll C$, with a nonlinear correction is of the order of $\Delta t^{3}$. The fit explains $82.4 \%$ of the variance in the data (Fig. 3.3, right panel).

What is the reason for the nonlinearities in $\Delta t, \sigma_{P}$ and $\sigma_{\Delta t}$ that become relevant at higher values of $\alpha$ ? Note that $\alpha$ has an upper limit at $2.7 \mathrm{~ms}$. At this point, a bifurcation occurs, i.e. the fixed point becomes unstable or even collides with the saddle point at the border of the basin of attraction, making them both vanish (see [36] for a discussion of these scenarios). We propose that the nonlinearities occur as $\alpha$ approaches the bifurcation point. This also explains why the effect of $\alpha$ is stronger for $\Delta t$ compared to the other parameters: Close to a bifurcation point, the transients that lead to the fixed point become longer and more variable. That does not affect the jitter of individual pools, nor the mean transmission delay, but it makes the time that the volley spends in the first few pools highly unpredictable, which increases the total timing error $\sigma_{T}$, and thus, indirectly also $\sigma_{\Delta t}$. 


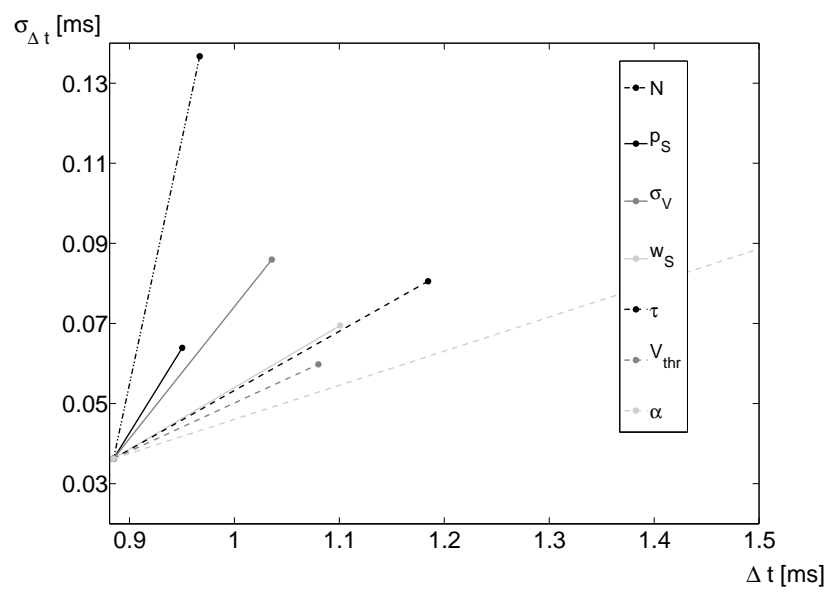

Figure 3.4.: Effect of the variation of the model parameters on the chain transmission characterized by $\Delta t$ and $\sigma_{\Delta t}$. The common starting point of each line corresponds to the combination of standard parameters listed in Table 3.1. Only one single parameter is varied at a time. Each line is drawn up to the point where the synfire chain becomes unstable. The only exception is the line for $\alpha$, which actually extends up to $\Delta t=5.44 \mathrm{~ms}$ and $\sigma_{\Delta t}=0.44 \mathrm{~ms}$.

\subsubsection{Effect of other parameters}

While the focus of this study is on the influence of $\alpha$, here we briefly discuss how other model parameters affect $\Delta t$ and $\sigma_{\Delta t}$. Although $\sigma_{\Delta t}$ increases with $\Delta t$ as $\alpha$ is increased, it is conceivable that these two measures are anticorrelated as another parameter is changed. We checked whether this is possible, with all those parameters that directly affect the dynamics of the network. Individual parameters to be changed are, apart from $\alpha$, the synaptic weights $w_{S}$, the connection probability $p_{S}$, the number of neurons in a pool $N$ and the membrane time constant $\tau$. While changing $p_{S}$ and $N, w_{S}$ is normalized to $0.345 / p_{S}$ and $100 / N$, respectively. Without this normalization, $p_{S}$ and $N$ would have similar effects as $w_{S}$, as the total number of presynaptic synapses of a neuron is changed.

Furthermore, the statistics of the membrane potential is important for the dynamics, described by $\langle V\rangle$ and $\sigma_{V} .\langle V\rangle$ enters the dynamics only by its distance from the firing threshold $V_{\mathrm{thr}}-\langle V\rangle$. This distance is most easily changed by modifying $V_{\mathrm{thr}} \cdot \sigma_{V}$, on the other hand, can only be modified by jointly changing $\lambda_{+}$and $\lambda_{-}$such that $\langle V\rangle$ stays constant. This is guaranteed if there is a certain linear relationship between the two rates [36].

We increased and decreased each parameter individually until the chain either breaks down (synchronizing effect too weak) or activity volleys form spontaneously without external stimulation (synchronizing effect too strong). Then, we calculated $\Delta t$ and $\sigma_{\Delta t}$ at parameter values slightly before one of the two events occur. Fig. 3.4 shows the results for all cases where $\Delta t$ increased. In all these cases, $\sigma_{\Delta t}$ increases as well. We also included $\alpha$ in the analysis for comparison. From Fig. 3.3, as well as from Eq. 3.16, the coefficients in Table 3.2 and the upper limit of $\alpha$ at $2.9 \mathrm{~ms}$, one can see that changing $\alpha$ increases $\Delta t$ up to $5.44 \mathrm{~ms}$ and $\sigma_{\Delta t}$ 

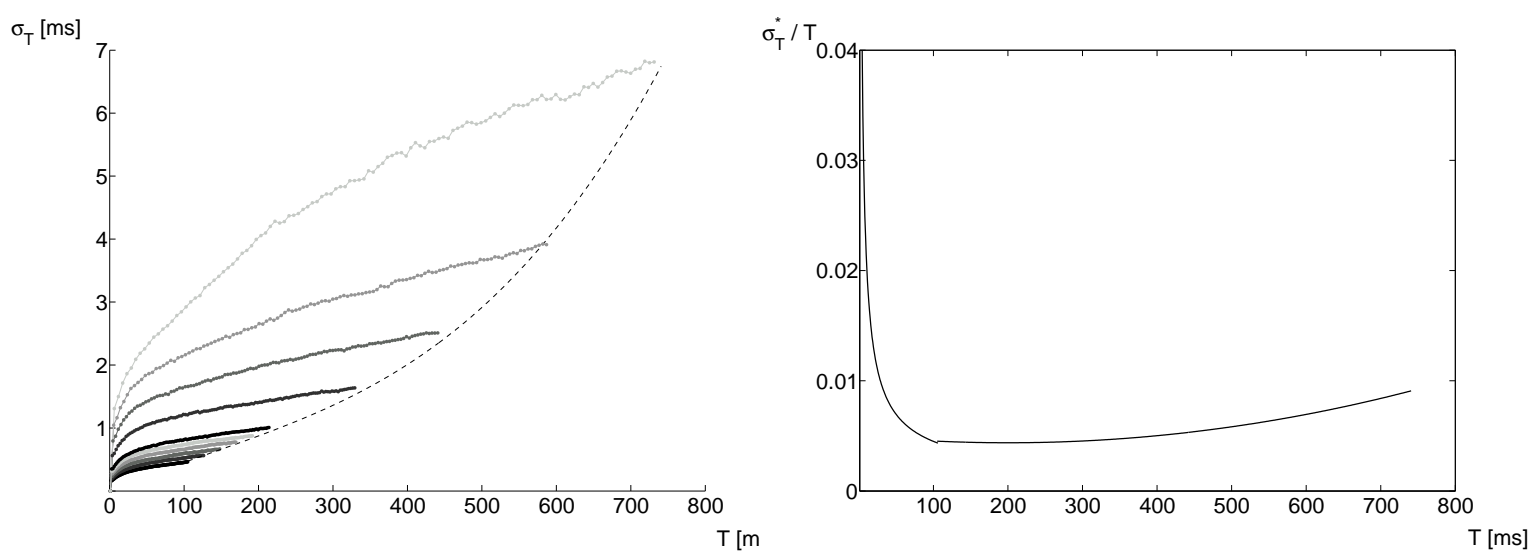

Figure 3.5.: Left: Timing error, e.g. variance of the total runtime of a chain $\sigma_{T}$ as a function of $T$ for various values of $\alpha$. The solid curves depict simulation data and the dotted line represents the optimal timing error $\sigma_{T}^{*}(T)$ from Eq. 3.19, It is close to the lower envelope of the simulation data. Right: Weber fraction $\sigma_{T} / T$ as a function of $T$ calculated from the lower envelope in Fig. 3.5. The U-shaped form of the Weber fraction that is known from the psychophysical experiments is reproduced.

up to $0.44 \mathrm{~ms}$. Thus, changing $\alpha$ makes it possible to increase $\Delta t$ much more than any of the other parameters, and, as seen from fig. 3.4, also at the lowest relative error.

Note that this analysis does not exclude the possibility of increasing $\Delta t$ without an increase of $\sigma_{\Delta t}$. For instance, one could increase $\alpha$ and also increase the number of neurons in a pool $N$ to compensate for the increase in $\sigma_{\Delta t}$. However, such a compensation would always result in a decrease of $\Delta t$ as well, limiting its possible range. Furthermore, Fig. 3.4 illustrates that the effect of any other individual parameter then $\alpha$ is rather limited. We conclude that one can only attenuate the increase of $\sigma_{\Delta t}$, but not abolish it completely over the full range of $\Delta t$.

Nevertheless, different combinations of parameters may still extent our results. For instance, it was shown in [187] that $\Delta t$ could be increases by a factor of 2 to 5 by changing the membrane time constant $\tau$ and the external input $I_{\text {ext }}$ (and thus, $V_{\text {thr }}-\langle V\rangle$ ), compared to an increase of merely $25 \%$ which we report here. What we have shown is that $\alpha$ is the most efficient parameter in changing $\tau$ in the sense that it induced the largest dynamic range for $\tau$ with the least relative increase in $\sigma_{\Delta t}$.

\subsection{Optimal temporal processing}

If there are different synfire chains $\mathcal{C}_{\alpha}$ with different transmission delays $\Delta t$, a given interval $T$ could be potentially represented by each of these chains. This redundancy opens the possibility to optimize the representation of time, in the sense of minimizing the timing error $\sigma_{T}$ for each interval $T$. The principle of optimization has already been used before in a more abstract pacemaker-accumulator system [97]. 
An interval $T$ is represented by a pool $i$ in the chain $\mathcal{C}_{\alpha}$ with transmission delay $\Delta t$. Only one of these parameters can be freely varied, the other one is fixed by the implicit equation $T=T(i, \Delta t) . \quad \sigma_{T}$ depends on both parameters, but much stronger on $\Delta t\left(\sigma_{T}=O\left(\Delta t^{3}\right)\right.$, Eq. 3.16 and Eq. 3.13) than on $i\left(\sigma_{T}=O(\sqrt{i})\right.$, Eq. 3.13). Thus, minimization of $\sigma_{T}$ requires using the lowest possible transmission delay $\min (\Delta t)$ and represent all intervals by the different pools $i$ of the fastest chain $\mathcal{C}_{\min (\alpha)}$.

This optimization results in a timing error that increases as $\sigma_{T}=O(\sqrt{T})$. This explains only part of the experimental results, namely the decreasing Weber fraction for short intervals. At longer times, optimization must be constrained such that a linear or faster-than-linear increase of $\sigma_{T}$ results. We propose that this constraint is given by a limited chain length. If a chain has a maximum of $L$ pools, the range of intervals that can be represented by the fastest chain $\mathcal{C}_{\min (\alpha)}$ has an upper limit of $T=\min (\Delta t) \cdot L$. For longer intervals, a chain with a higher $\Delta t$ and thus, a higher $\sigma_{T}$ must be used. As the timing errors are dominated by the third-order dependency on $\Delta t$, the constraint optimization problem can be formulated as

$$
\Delta t^{*}(T)=\min \{(\Delta t) \mid T \leq \Delta t \cdot L\} .
$$

where $\Delta t^{*}(T)$ is the optimal choice of $\Delta t$ for representing the interval $T$. In the simplest case, assuming a smooth distribution of $\Delta t$, every interval can be exactly represented by an individual synfire chain, and Eq. 3.17 simplifies to

$$
\Delta t^{*}(T)=\frac{T}{L}
$$

Taken together, Eqs. 3.14, 3.16, and 3.18 yield the optimal form of the timing error

$$
\sigma_{T}^{*}(T)=\left\{\begin{array}{cl}
\sigma_{\Delta t}(\min (\Delta t)) \cdot \sqrt{\frac{T}{\min (\Delta t)}} & \text { for } T \leq \min (\Delta t) \cdot L \\
\frac{A}{L^{5 / 2}} T^{3}+\frac{B}{L^{3 / 2}} T^{2}+\frac{C}{\sqrt{L}} T & \text { otherwise }
\end{array}\right.
$$

with the coefficients $A, B$, and $C$ from Eq. 3.16 and Table 3.2 (final row). For $T>\min (\Delta t) \cdot L$, the timing error is entirely dominated by $\sigma_{\Delta t}$. To minimize $\sigma_{\Delta t}$, always the final pool $L$ of each chain is used (Eq. 3.18), so the $\sqrt{T}$ dependency degenerates into $\sqrt{L}$, which is constant for all $T$ and enters the coefficients of the third-order polynomial. $\sigma_{T}^{*}$ depends mostly linear on $T$, with small nonlinear corrections of the order of $T^{3}$, just as $\sigma_{\Delta t}$.

In Fig. 3.5 (left), we have plotted $\sigma_{T}(T)$ for a number of different $\mathcal{C}_{\alpha}$ from simulations together with the optimal $\sigma_{T}^{*}(T)$ from Eq. 3.19, which is close to the lower envelope of the simulation data. Both from Fig. 3.5 and Eq. 3.19, one sees the three regimes that are found in the experiments: $\sigma_{T} \propto \sqrt{T}$ for $T \leq \min (\Delta t) \cdot L, \sigma_{T}$ increasing faster than linear for $T \gg \min (\Delta t) \cdot L$, and an intermediate regime where the linear term in Eq. 3.19 dominates and $\sigma_{T} / T$ is approximately constant. Consequently, the Weber fraction $\sigma_{T} / T$ calculated from Eq. 3.19 follows the experimentally observed U-shaped form (Fig. 3.5, right). 


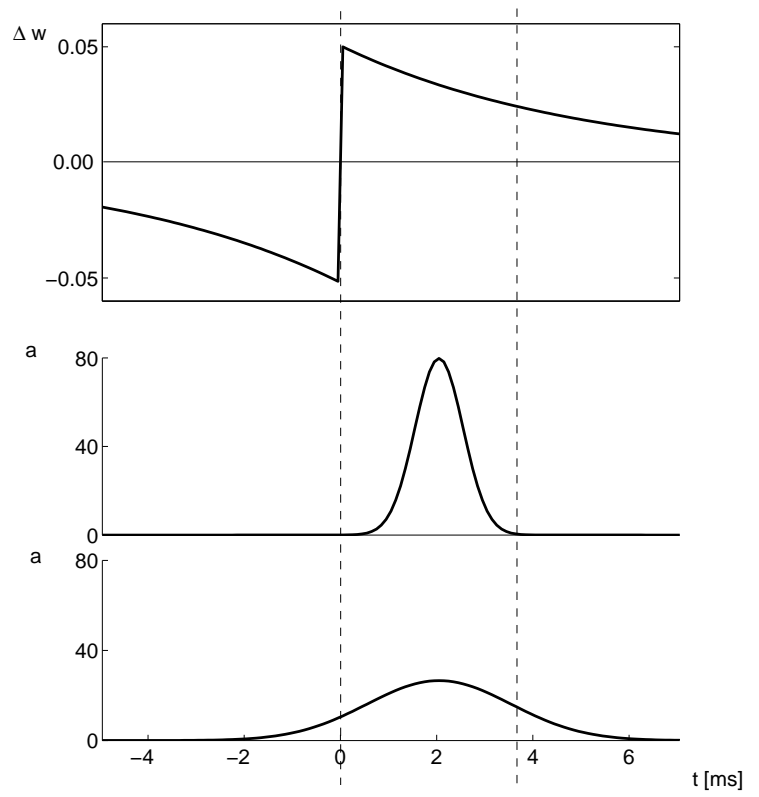

Figure 3.6.: Illustration of the STDP learning curve (top panel), alinged to two spike time distributions from chains of different speed. $t$ denotes the relative time between the presynpatic and postsynaptic spike (cf. Eq. 3.6). If the means are aligned, the different standard deviations cause a relative advantage for the narrower distribution. This is because the peaked distribution has a larger overlap within the area in between the dashed lines than the broader one.

\subsection{Optimization by competitive STDP learning}

In the preceding section, we have shown how the observed timing errors can be explained by an optimal selection of synfire chains. In this section, it remains to be shown how this selection is neuronally implemented and a unique representation of time is formed from the different chains. Here we show how both issues can be resolved if the chains project to a set of readout neurons and the synaptic weights of these projections are subject to both STDP and homeostatic plasticity.

To see how the representation of an interval $T$ can be learned in this framework, consider the following experiment (cf. Fig. 3.8, top panel). At time $t_{0}$, a stimulus $S_{0}$ (called initiation stimulus) is given that activates the first pool of all chains and makes the volleys travel along the chains with their respective speeds. At a second time $t_{1}=t_{0}+T$, another stimulus $S_{1}$ (training stimulus) activates the readout neurons. Around this time, inputs from the chains also arrive. If this experiment is repeated, the connections to those pools of the chains will grow that were active slightly before the stimulus $S_{1}$. Eventually, a certain set of readout neurons will fire at time $t_{1}$ even in the absence of stimulus $S_{1}$ if the stimulus $S_{0}$ is given at time $t_{0}$. 


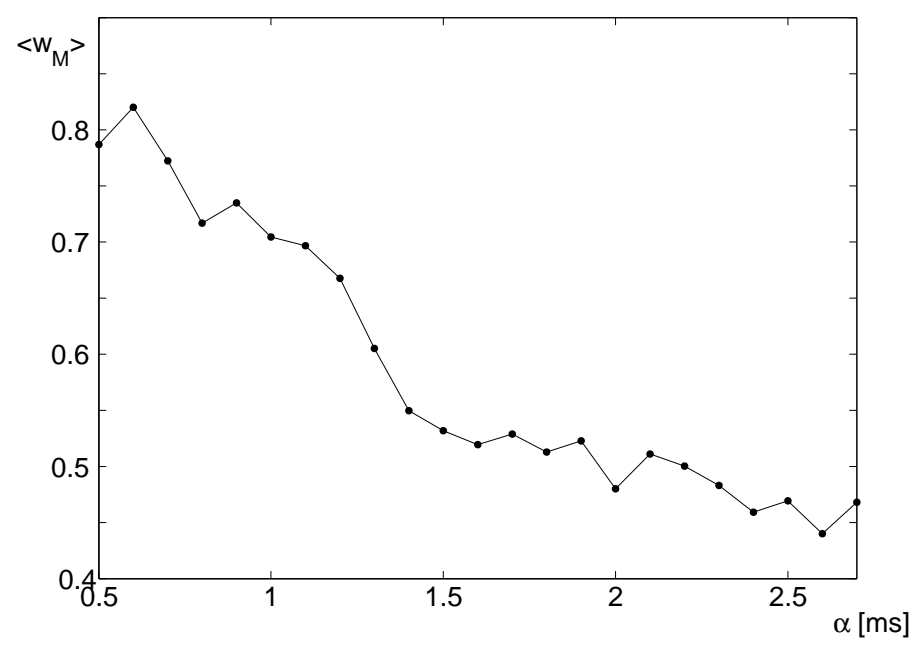

Figure 3.7.: Mean synaptic weights $\left\langle w_{M}\right\rangle$ of the connections from a pool (shown is pool number 50) to a readout neuron after 50 presentations of the corresponding interval. The graph is obtained by varying the PSP rise time $\alpha$ used in the chain connections. All weights were initialized by the valus 0.3 .

Using this paradigm, we first show in Sec. 3.5.1 that the effective learning rate of STDP is higher for inputs from a fast synfire chain with a peaked spike time distribution compared to the input from a slower one, meaning that the mean synaptic weight of the connection is higher after a given number of trials. Second, we consider a set of readout neurons that is connected to two different chains, one with a low $\alpha\left(\mathcal{C}_{\alpha_{1}}\right)$ and one with a higher one $\left(\mathcal{C}_{\alpha_{2}}\right)$. If the input from the two chains arrives at the same time, we can test the optimal selection of chains (Sec. 3.5.2): The timing errors $\sigma_{T}$ in the readout neuron should be comparable to the smaller errors in chain $\mathcal{C}_{\alpha_{1}}$. If the inputs arrive at different times, we can test for the unique representation (Sec. 3.5.3): Even if a training stimulus is given at both arrival times, the readout neurons should learn to fire only once. Both of these properties are confirmed and can be extended to a scenario with several input chains.

\subsubsection{Effective learning rate depending on timing errors}

Before we explain the mechanism that brings about optimality and uniqueness of the representation, we elaborate on how the effective learning rate of STDP depends on the temporal distribution of the presynaptic spikes. For this purpose, we consider only connections from a single synfire chain and neglect homeostatic plasticity. Furthermore, we assume for simplicity that the postsynaptic spikes in the readout neurons (i.e. the training stimulus $S_{1}$ ) occur at a fixed time $t_{1}$ without any jitter. Assuming for example a Gaussian distribution of $S_{1}$ around $t_{1}$ does not change the following argument, as it only increases the variance of the relative time between the pre- and postsynaptic spikes. 
To illustrate the effects of different temporal spreads in the presynaptic spike times, consider two Gaussian firing time distributions with the same mean spike time $t^{*}$, but different variances (Fig. 3.6). The temporal asymmetry in STDP, and the exponential decrease of its efficiency with increasing $|t|$ (cf. Eq. 3.6 and Fig. 3.6, top panel) are the reasons why the width of the input distribution influences the effective learning rate: The trial average of this rate is given by the convolution of the weight increase at a given value of $t$ and the distribution of $t$. From Fig. 3.6, one can see that the area under a peaked distribution largely overlaps with the area under the positive branch of the learning curve up to its half width at half maximum, marked by the two dotted lines. The mean learning rate is therefore larger for a more strongly peaked input distribution than for a broader one, as the latter overlaps more with areas of less positive increase, and even with the negative branch of the STDP curve.

In Sections 3.3.2 and 3.4, we have shown that the temporal spread of the firing times in synfire chains increases with $\alpha$. Thus, by the preceding argument, the mean weight from chain neurons to a readout neuron after a given number of learning trials should decrease with increasing $\alpha$. To test this assumption, we fully connected all neurons in the pool number 50 of a chain to all the readout neurons and performed the simulated learning experiment 50 times for each different value of $\alpha$. $S_{1}$ was presented $3 \mathrm{~ms}$ after the mean firing time of the chain neurons in pool 50. Fig. 3.7 shows that the synaptic weights after the learning trials indeed decrease for increasing $\alpha$. This result is independent of the pool number and of the $\alpha$ used in the connections to the readout units.

\subsubsection{Optimal selection of synfire chains}

If a readout neuron is connected to two synfire chains $\mathcal{C}_{\alpha_{1}}$ and $\mathcal{C}_{\alpha_{2}}$ (Fig. 3.1), its firing pattern may be shaped by the input from both of them. The combination of STDP and homeostatic plasticity introduces synaptic competition among the chains: The weights are increased by STDP with a different effective rate (cf. Sec. 3.5.1), but the rate of compensation by homeostasis is the same for both chains (cf. Eq. 3.7). Thus, the faster chain $\mathcal{C}_{\alpha_{1}}$ with the higher STDP rate will win the competition and dominate the firing pattern of the readout neuron.

First, we study the effect of this competition for the case that both inputs arrive at the same time. This means that the neurons which are connected to the readout neuron have the same mean firing time in both chains, marked by the dotted line in Fig. 3.8 (top panel). $S_{1}$ is given slightly before this mean. If the input from chain $\mathcal{C}_{\alpha_{1}}$ dominates the firing pattern of the readout neuron, its timing error $\sigma_{T}$ should be lower compared to the case where it only receives input from the slower chain $\mathcal{C}_{\alpha_{2}}$. This corresponds to a selection of the optimal chain $\mathcal{C}_{\alpha_{1}}$.

To test if this selection takes place, we connected the readout neurons to one pool of chain $\mathcal{C}_{\alpha_{1}}$ and to another pool of $\mathcal{C}_{\alpha_{2}}$, chosen such that the mean firing times of the two pools are at $68 \mathrm{~ms}$, which is the largest interval in the chain $\mathcal{C}_{0.3} . \alpha_{1}$ was fixed to $0.5 \mathrm{~ms}$, while $\alpha_{2}$ was varied from 0.3 to $2.5 \mathrm{~ms}$. If there is no pool which is activated exactly at $68 \mathrm{~ms}$, we chose the one that is closest to this time and shift the starting time of the respective chain. This shift is always less than a millisecond. Without such a shift, the deviations of the means from $68 \mathrm{~ms}$ would be another source of timing errors. For every $\alpha_{2}$, we performed 10 sets of trials with 300 trials each. The first 200 trials were used to modify the synaptic weights to the readout 

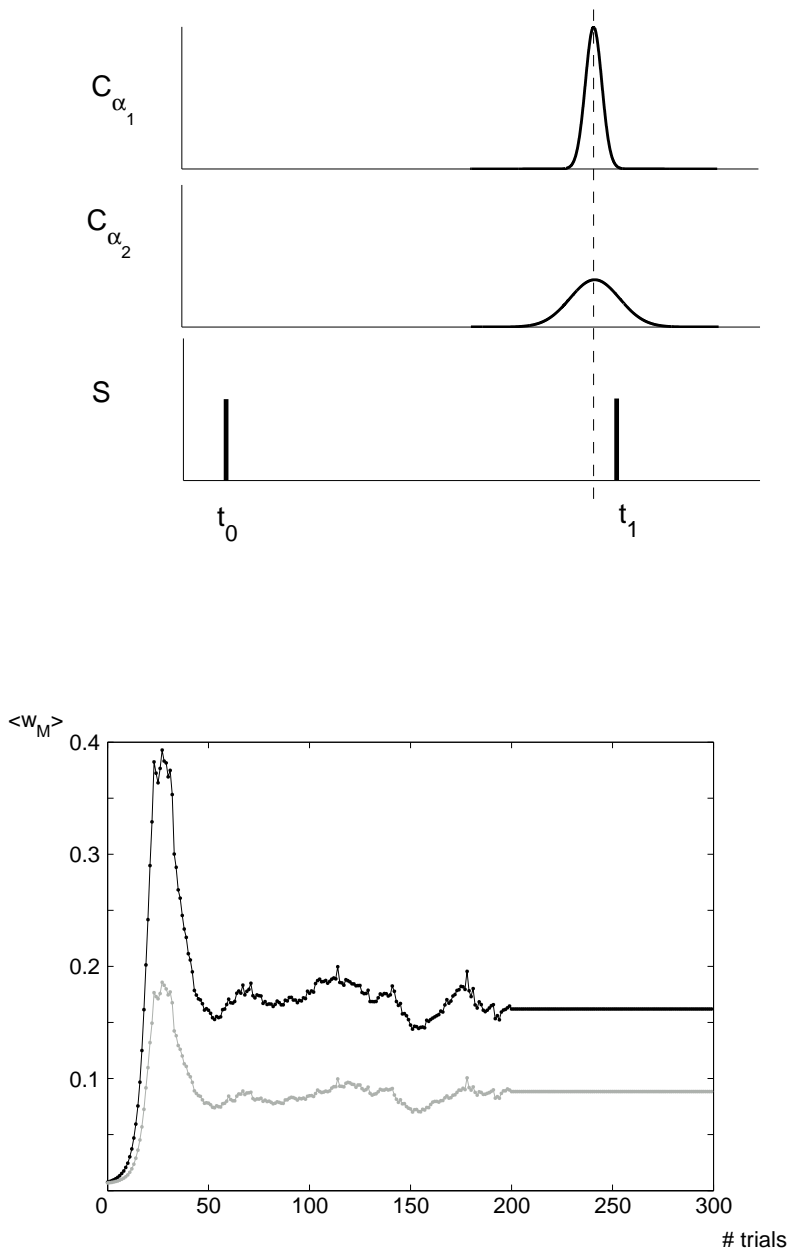

Figure 3.8.: Top: Illustration of the activation pattern in the readout neurons $\mathcal{M}$ that are driven by the synfire chain $\mathcal{C}_{\alpha_{1}}$ and $\mathcal{C}_{\alpha_{2}}$, cf. Fig. 3.1. At time $t_{0}$, the initiation stimulus $S_{0}$ activates the first pool of each chains and causes volleys along all the chains with their respective speeds. At time $t_{1}=t_{0}+T$, another stimulus $S_{1}$ that indicates the termination of the temporal interval activates the readout neurons. Around this time, inputs from the chains also arrive. The learning rule (see Fig. (3.6) increases the connections to those pools that were active slightly before the stimulus $S_{1}$. Bottom: Mean synaptic weight $\left\langle w_{M}\right\rangle$ of all connections from $\mathcal{C}_{0.5}$ (black curve) and $\mathcal{C}_{1.5}$ (gray curve) to the readout neurons $\mathcal{M}$ as a function of the number of trials. During the first 200 trials, the weights are modified by STDP and homeostatic plasticity, while the final 100 trials are used to calculate $\sigma_{T}^{M}$. Ultimately, the connections to the faster chain $\mathcal{C}_{0.5}$ are much stronger than those to the slower chain $\mathcal{C}_{1.5}$. 


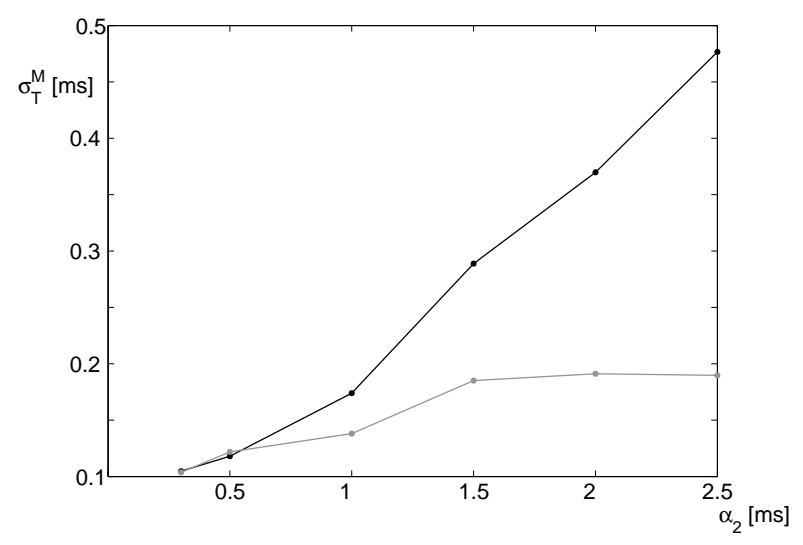

Figure 3.9.: Timing error $\sigma_{T}^{M}$ in the readout neurons $\mathcal{M}$ as a function of $\alpha_{2}$ (cf. Fig. 3.6). The grey curve shows $\sigma_{T}^{M}$ for $\alpha_{1}=0.5$ and the black curve for $\alpha_{1}=\alpha_{2}$. For the case of $\alpha_{1}=0.5$, the timing error is dominated by the input from $C_{\alpha_{1}}$ and much lower compared to $\alpha_{1}=\alpha_{2}$ for $\alpha_{2}>0.5$.

neurons by STDP and homeostatic plasticity, while in the last 100 trials, the timing error $\sigma_{T}^{M}$ in the readout neurons was calculated without any further learning. $\sigma_{T}^{M}$ is defined in the same way as $\sigma_{T}$ for the synfire chains (cf. Sec 3.3.2), just using the neurons in the readout network instead of those in a pool. The target rate is set to $a_{\text {goal }}=2$ spikes per neuron and trial, one spike from $S_{1}$, and another from the chain input. The chain input always arrives before stimulus $S_{1}$, which is set to be strong enough to make the neurons fire even shortly after a spike induced by the chain input.

Fig. 3.8 (bottom panel) shows the synaptic competition between the two chains. Initially, STDP dominates the learning dynamics and increases the two types of connections according to the speed of the input chain (cf. Sec. 3.5.1). This produces an overshoot over the firing rate over the target rate $a_{\text {goal }}$, and homeostatic plasticity is finally strong enough to bring the weights down again. Different from STDP, the homeostatic learning rule is blind to the different speeds of the input chains, but reduces the weights only according to their current strength (cf. Eq. 3.7). Thus, the difference between the connections from $C_{\alpha_{1}}$ and $\mathcal{C}_{\alpha_{2}}$ remains as the mean firing rate approaches $a_{\text {goal }}$, resulting in partial suppression of the input from the slower chain $\mathcal{C}_{\alpha_{2}}$.

In Fig. 3.9, we show the timing error $\sigma_{T}^{M}$ resulting from this synaptic competition as a function of $\alpha_{2}$. For comparison, we conducted another simulation where $\alpha_{1}=\alpha_{2}$, instead of being fixed to $0.5 \mathrm{~ms}$. In this case, the timing error is fully determined by the input from $\mathcal{C}_{\alpha_{2}}$. One sees that the error is much lower in the case of synaptic competition for all $\alpha>0.5$. For $\alpha=0.3$, on the other hand, the errors in both cases are comparable, as $\mathcal{C}_{\alpha_{2}}$ is the faster chain now and thus dominates $\sigma_{T}^{M}$.

\subsubsection{Unique representation in readout neurons}

We now study the second case that is possible with the connectivity depicted in Fig. 3.1, $\mathcal{C}_{\alpha_{1}}$ having an different mean firing time from $C_{\alpha_{2}}$. In this case, the question is how to prevent 
the readout neuron to respond to two different time intervals. If the system is exposed to two training stimuli $S_{1}$ and $S_{2}$ slightly before each of the chain firing means, both intervals could be trained to the same neuron (see Fig. 3.10, top panel). Thus, one could not tell which of the two times has elapsed upon firing of this neuron.

Such a double training of the same neuron is also prevented by synaptic competition. If the same neuron fires two times responding to a single training stimulus, the firing rate is higher than the target rate $a_{\text {goal }}$. This leads to a compensation that weakens the connections to both chains, but finally leads to a suppression of the input from the slower one, as its connection was weaker in the first place (cf. Sec. 3.5.1). The readout neurons only responds to the interval represented by the faster chain and the unique representation is restored.

As a test of this reasoning, we use the same simulation as in Sec. 3.5.2, with the only difference that the connecting pool of $\mathcal{C}_{\alpha_{2}}$ is chosen to match a certain mean firing time different from the one of $\mathcal{C}_{\alpha_{1}}$. This interval between the two mean firing times is now varied instead of the $\alpha$, which are kept constant to $\alpha_{1}=1.5 \mathrm{~ms}$ and $\alpha_{2}=0.5 \mathrm{~ms}$.

Fig. 3.10 (bottom panel) shows the mean firing time of the readout neurons $T_{M}$ as a function of the varied mean firing time $T_{\alpha_{2}}$ in $C_{\alpha_{2}}$. For a unique representation, $T_{M}$ should either be identical to $T_{\alpha_{2}}$ (gray dotted diagonal) or to the fixed firing time of $\mathcal{C}_{\alpha_{1}}$ (black horizontal line). The figure shows that such a unique representation is maintained for all $T_{\alpha_{2}}<T_{\alpha_{1}}$. For $T_{\alpha_{2}}$ after the mean firing time of $C_{\alpha_{1}}$, the situation is less clearly described. While $T_{M}$ faithfully follows $T_{\alpha_{2}}$ for $20 \mathrm{~ms}$ after $T_{\alpha_{1}}$, it jumps between $T_{\alpha_{1}}$ and values between $T_{\alpha_{1}}$ and $T_{\alpha_{2}}$ for higher $T_{\alpha_{2}}$, indicating a significant number of spikes at both of these times. This can be explained if one considers that the timing error increases with $\sqrt{T}$ (Eq. 3.14). If $T_{\alpha_{1}}$ stays constant while $T_{\alpha_{2}}$ increases, the difference in the timing errors decreases, and thus, the synaptic weights from both chains will be more similar. We conclude that a unique representation is possible for all $T_{\alpha_{2}}<T_{\alpha_{1}}$. For $\alpha_{2} ; \alpha_{1}$, however, this will be the most common situation, as the range of time intervals in $\mathcal{C}_{\alpha_{2}}$ is smaller than $\mathcal{C}_{\alpha_{1}}$ (cf. Fig. 3.5) and thus, $T_{\alpha_{2}}>T_{\alpha_{1}}$ rarely occurs.

\subsection{Discussion}

At first glance, the $\sqrt{T}$ dependency of the timing errors in a synfire chain seems to be incompatible with the experimental results of a constant or even increasing Weber fraction, which is a problem shared by many other models of timing. However, we identified a mechanism that makes the additional error plausible, namely the superlinear increase of the timing error with the transmission delay. Thus, we do not need to postulate any ad hoc assumptions about the scalar property, but could explain both the linear and faster-than-linear error increase from a constraint optimization process. Moreover, we found a neuronal implementation of this optimization by synaptic plasticity that also solved the problem of combining output from the various synfire chains to a unique representation of time.

A central assumption of our work is the limitation of the number of pools in a synfire chain. One possible reason why such a limit should apply is provided by a capacity argument. Synfire chains have been proposed to model the function of the cortical column [70, 14], a structure 

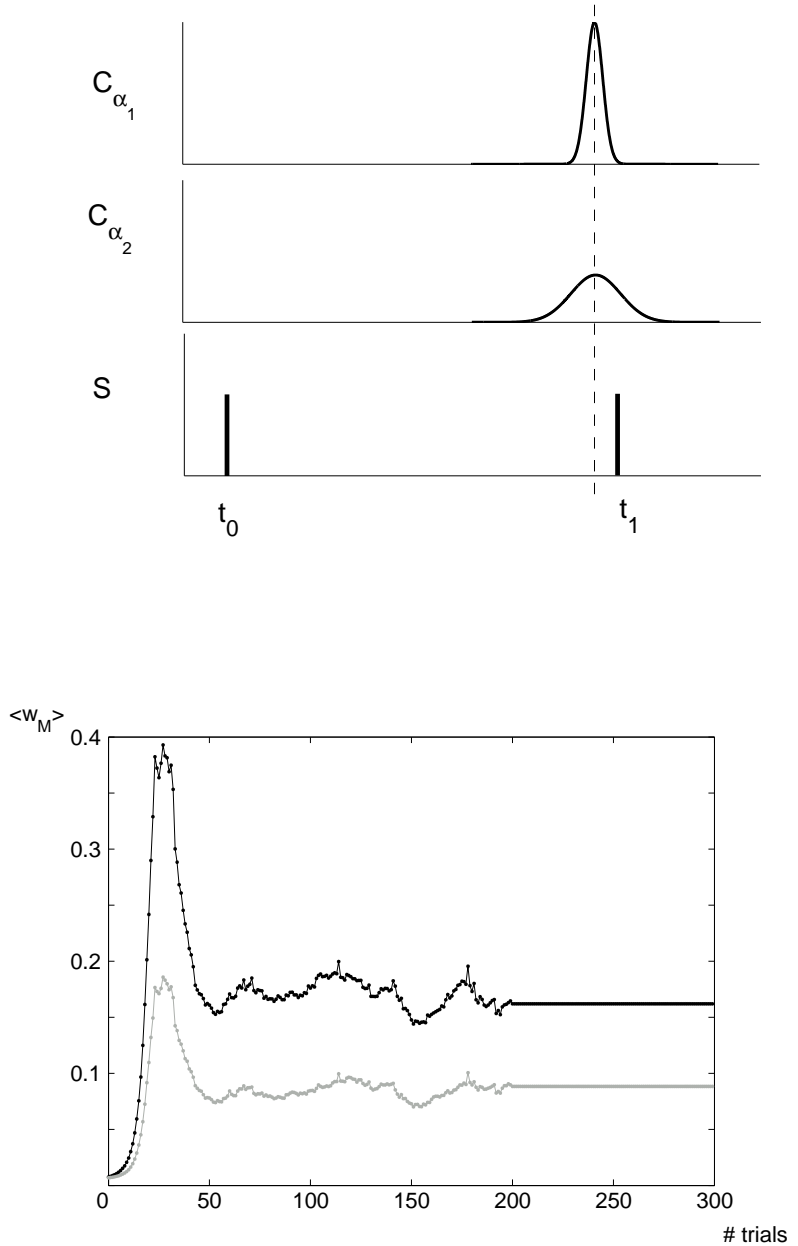

Figure 3.10.: Top: Illustration of the activation pattern in the readout neurons $\mathcal{M}$. Same as in Fig. 3.8 (top panel), except for the different mean firing times of $\mathcal{C}_{\alpha_{1}}$ and $\mathcal{C}_{\alpha_{2}}$ and two training stimuli occurring at $t_{1}$ and $t_{2}$, which are slightly before the temporal means of the two chains. Bottom: Mean firing time of the readout neurons $T_{M}$ as a function of the mean firing time $T_{\alpha_{2}}$ in $\mathcal{C}_{\alpha_{2}}$. The firing time of $\mathcal{C}_{\alpha_{1}}$ is kept constant to $T_{\alpha_{1}}=140.2 \mathrm{~ms}$. For $T_{\alpha_{2}}<T_{\alpha_{1}}$, the readout neurons fire at about the same time as the neurons in chain $\mathcal{C}_{\alpha_{2}}$. 
containing $10^{4}$ to $10^{5}$ neurons. A pool size of the order of $10^{2}$, comparable to the size of a minicolumn, has been shown to be necessary for stable propagation of the chain [70, 14, 37]. Thus, the number of pools in the chain is constrained to the order of $10^{2}$ to $10^{3}$. Of course, each of the neurons in a column could participate in multiple chains, but the capacity of network for synfire chains has been found to be limited [70, 14, and it has been proposed that this capacity only allows for representation of events of durations up to 1s [70]. However, all these studies assume a transmission delay of about $1 \mathrm{~ms}$, which is true only for the fastest chains in our framework. Possible delays up to $6 \mathrm{~ms}$ do not seem to enable computations much above the range of one second, due to the increase in timing errors. In order to compensate these errors, an increase of the width of the chain were necessary which in turn reduces capacity. The one second range has also been found in physiological experiments with precise spiking patterns [76, although the results of this study are disputed.

Another argument for a constrained pool number relates to the formation of synfire-like structures with a distribution of transmission delays. It has been shown that such structures might emerge from STDP learning in recurrent networks [82. In this study, the number of neurons in each of the "polychronous groups" was less than 20 in the mean, in a network of 1000 neurons. Much larger networks tended to become unstable. Although it seems to be possible to stabilize such groups by external guidance [82, 27, problems of unstable connections are likely to put a further constraint on the length of a chain.

Apart from the limited chain length and the general connectivity of several synfire chains projecting onto readout neurons, many of the assumptions used in this model can be relaxed. First of all, the synfire chains are allowed to contain a certain amount of recurrent connections, which introduce an additional source of error, but do not destabilize the propagation of activity [70]. Second, it is not necessary to prewire the connections to the readout neurons in the way we have used here. Rather, this connectivity will arise spontanously from an initally random wiring because of the synaptic competition. Consider each readout neuron being initially connected randomly to a certain fraction of the pools in a single chain. STDP will then only enhance the connections to those pools that are active slightly before the stimulus. But at the same time, homeostasis weakens all the connections, including those which were not enhanced. As a result, connections which does not fit into the scheme we have proposed for learning temporal representations end up to be very weak, and might also be removed by means of synaptic turnover. Finally, the model is also robust to changes in the properties of the noise. Introducing a finite correlation length into the noise only adds a constant to the timing error and does not change the form of the error function. And even if the $\sqrt{T}$ law in the timing errors of the individual chains changed due to some properties of the noise, one would expect that this affected all of the synfire chains alike. So the selection of optimal chains would still work in this case and the U-shaped form of the Weber fraction would be preserved.

The combination of synfire chains and the readout network with plastic connections opens the possibility to explain some further phenomena of temporal processing. For instance, it has been shown that the subjective length of an interval depends on attention: If a timing task has to be performed in parallel with a second, non-temporal task, the duration of the interval 
is systematically underestimated [61]. This can be explained by our model if attention is modeled by the level of activation in the synfire neurons. The mean membrane potential $\langle V\rangle$ is increased, and thus, the difference $\langle V\rangle-V_{\mathrm{thr}}$ is decreased. This decreases $\Delta t$ (cf. Sec. 3.3.2 and [187]). Conversely, decreased attention due to a parallel task decreases $\langle V\rangle$ and slows down the chain, resulting in an underestimation of intervals.

Moreover, temporal representations are subject to selective learning: If a participant is trained with stimuli of a certain duration, discrimination of that duration is improved after training, but this effect does not generalize to different intervals [28]. This is also readily explained in the framework of our model: Training of a specific duration strengthens the connections of the responsible readout neurons with the pools that are active at this time, and suppresses the random connections to other pools by means of synaptic competitions. The learning experiment described in Sec. 3.5 can also be related to the paradigm of classical conditioning, where the initiating stimulus $S_{0}$ corresponds to the conditioned stimulus (e.g., the ring of a bell or a flash of light) which can be learned to predict the unconditioned stimulus (e.g., food or an airpuff), corresponding to the training stimulus $S_{1}$ in our case. This may also solve the problem that learning seems to occur on time scales that are much longer than those of the STDP learning rule [159]. Note that there is no need to assume that $S_{0}$ only activated the synfire chains and $S_{1}$ only the readout network. If there is no such distinction, $S_{1}$ would both mark the end of a first interval and the beginning of another, starting off a new volley of synfire activity. In this way, the apparent "reset" of the timing system could be explained [28].

Based on our results and earlier descriptions of neuronal structures and connections that might be relevant for temporal processing [24], we sketch a hypothetical architecture of our model in the brain: Synfire chains are present in all areas of the neocortex, performing computational tasks like pattern storage [70, 14] or compositional binding [68]. They have different transmission delays that might have been shaped during their formation by the time scale of the task they perform. As a by-product of their usual computation, the chains encode the temporal information of a real or imagined event. These distributed time representations are then projected onto a central readout network that is located in the striatum [24]. Distortions in the level of dopamine, as induced by certain drugs or Parkinson's disease will strongly affect the function of the readout neurons and thus, also the timing performance [24, 148]. The connections from the chains to the readout neurons are initially randomly distributed and are shaped by synaptic plasticity to implement an optimal, unique representation of time. Nevertheless, input from suboptimal chains will not be entirely suppressed, so the random connectivity remains an additional source of errors that can be further reduced by training.

Note that within this framework, it is improbable that there is a separate chain for each conceivable time interval, as we have assumed in Sec. 3.4. More likely, there will we a finite set of chains that represents an entire range of durations by using more than just their final pool. Of course, this violates the optimality condition Eq. 3.18 to some extent, introducing another error source. More specifically, the timing errors will not increase as smoothly as Eq. 3.19 implies, but there will be jumps in the error whenever a certain chain has reached its final pool and longer intervals must resort to the next chain. Interestingly, such jumps have 
indeed been observed in psychophysical experiments after excessive training [102]. It seems that those jumps are normally masked by noise that is reduced by training. One possible source of this noise might be the random connectivity to the readout neurons, which can be refined by plasticity (see above). Furthermore, it is conceivable that the transmission delay of the chains can be fine-tuned by slightly changing the activity level [187]. This might explain short-term adaptation effects which occur at the presentation of sequences [17]. Mechanisms that are not contained in the current form of the model include memory and decision.

A quantitative view on the Weber fraction calculated from our simulation data (cf. Fig. 3.5, left) reveals that its value of 0.5 to $4 \%$ of the represented interval is too low compared to the psychological experiments, which report values between 2 and $20 \%$ [58, 39, 54]. This is due to a relatively low level of synaptic noise $\left(\sigma_{V}=1.4 \mathrm{mV}\right.$ in our study compared to e.g. 2.85 $\mathrm{mV}$ in [37]). We conducted tests of whether this level can be increased while maintaining stable propagation of chains. Preliminary results show that this is possible by compensation of the increased noise with increasing both synaptic weights $w_{S}$ and the firing threshold $V_{\mathrm{thr}}$. Using these measures, the Weber fraction is increased to values between 3 and $9 \%$. A full exploration of the synfire parameter space is beyond the scope of this research, but it seems that at least the lower range of the Weber fractions experimentally observed can be obtained within the biologically realistic range of parameters. Some additional error sources have been mentioned in this section.

Finally, we note that our framework is not necessarily limited to synfire chains. Any timing system with a limited dynamic range will show a similar effect, given that this range can be extended at the cost of a superlinear increase in the timing error. The optimization scheme and readout network will be the same in this case. It seems worthwhile to check this properties for neurocomputational models of timing such as state-dependent networks [25, 27], ramping activity [40] or the striatal beat model [119]. The convergence of evidence from psychology and neuroscience is likely to decide which classes of models are able to explain how our brain tells time. 


\section{Information-theoretical analysis of time perception}

In Chapter 3, we described a neurocomputational model which is capable of reproducing an experimentally observed error function when subject to constrained optimization. Here, we present a statistical framework which can be used to test this predictive property for arbitrary models of time perception: The optimal, i.e. minimal error of a time estimate can be computed as the Cramer-Rao bound when the underlying process is interpreted as a stochastic process (cf. Section 2.31). We study here the case of Gaussian stochastic processes with temporal information in the mean, the variance, and the covariance structure. For single processes, we find a hierarchy of temporal information in the moments, under quite natural assumptions for their temporal evolution: The lower bound for the timing errors scales with the square root of the duration of the estimated interval if only the information from the mean is used, but is linear in the duration when relying on the variance alone, and even increases exponentially with the interval duration when using only the covariance.

Furthermore, two case studies of multiple stochastic processes suggest that the scaling behavior of the timing error is not changed when there is more then one process available at the same time. We also formulate the synfire chain model in terms of a stochastic process, and show that the optimal results we obtained in the preceding chapter are also optimal in this more mathematical sense.

\subsection{Introduction}

Precise representations of time are of crucial importance for a wide range of brain functions such as speech recognition and the planning and execution of coordinated movements. To psychologists, the estimation of time has been a major subject of the study of qualitative properties of information in the brain. A recurring result of the experiments was that the just noticeable difference between two temporal intervals increases linearly with the duration of the intervals, which is referred to as the Weber-Fechner law, or the "scalar property" [58] (cf. Section 2.1.2.2). This is a result that was originally obtained for directly observable sensory inputs such as sound intensity. This analogy between the perception of time and the perception of other physical quantities point to shared statistical properties between the different domains. However, the origin of this law for time perception remains unknown and has been subject to modeling approaches such as scalar expectancy theory [55]. More recently, also neuroscientists became interested in the topic and proposed models for the neural substrate of temporal processing, such as ramping firing rates [117, 40, 41, neural oscillators read out by coincidence detectors [119] or synfire chains [66] (cf. Section 2.2.3). 
While the underlying neural mechanisms and the conceptual questions on temporal information still remain subject of an ongoing debate, more abstract viewpoints from statistical estimation theory have recently been added. They allow the formulation of the underlying question with a precision and simplicity so far unseen in this field [5]. From this point of view, the stochasticity of brain processes is not merely a source of noise which obscures temporal information of the underlying deterministic process, but may also be an actual source for this information. To show this, Ahrens and Sahani [5] used a process with zero mean, where the only time-dependent properties was an exponentially decaying covariance. They showed that the interval between two points in time can be estimated from such a process. This is an example how the transition from a computational towards a mathematical model increases explanatory power, while inheriting features of models that were developed empirical and computational psychology during the last decades.

However, while Ahrens and Sahani constrained themselves to the temporal information in the covariance, general neural processes will also contain such information in the mean and the variance. This complies with the intuitive notion of time as a Janus face of both progress and decay: The systematic change of states in the brain such as spike patterns or firing rates in response to external stimuli or ongoing thoughts contains information about elapsed time. But such information is also contained in the decay of such neuronal signals, or signal correlations over time. Here, we extend the stochastic framework [5] by these dimensions and compare the relative information content from the first and second moments of a stochastic process. By analyzing the scaling of the minimal obtainable timing errors with the duration, we can compare the optimal estimations to psychophysical results.

First, we study the case of single Gaussian process with temporal changes in both the mean and the variance and covariance and use Fisher information to compute the theoretical lower bound of timing errors which gives rise to a finite just noticeable difference. Under quite generic assumptions (mean and variance linearly increasing in time, exponentially decaying correlations), we find a hierarchy of temporal information in those three moments: The lower bound for the timing errors scales with the square root of the duration of the estimated interval if only the information from the mean is used, but is linear in the duration when relying on the variance alone, and even increases exponentially with the interval duration when using only the covariance.

Second, we extend this framework to the case where multiple processes with different time scales are available. This allows to re-analyze the studies of Ahrens and Sahani [5], as well as our own model based on multiple synfire chains [66] (cf. Chapter 3 in greater detail. We find that also in the case of multiple processes, the standard deviation of the time estimate increases exponentially when based on exponentially decreasing covariance. This is irrespective of the details of the model and implies that estimation from covariance alone can only be successful for times that are small compared to the time scales of the used processes. Furthermore, we confirm that the optimal selection for time perception based on multiple synfire chains (cf. Section 3.4) is also optimal in the sense that the resulting timing errors are minimal. 
This approach may lay a ground for a comparison of the performance of the many competing models and thus experimentally constrain the class of plausible models of timing. Furthermore, seemingly different neural processes, such as synfire chains [66] and ramping activity [117, 40, 41], appear much more similar in the light of estimation theory. In this way, common optimality principles underlying temporal perception become apparent that may otherwise be masked by the neuronal details.

\subsection{Estimation of temporal intervals}

For the estimation of the length of a temporal interval between two successive times $t_{1}$ and $t_{1}+$ $\Delta t$ a Gaussian process $y(t)$ provides a sufficiently abstract model of the cerebral mechanisms for time estimation [5]. It may find realizations e.g. in a sequence of subsets of neurons that are maximally active [66], or a randomly increasing firing rate [117]. The values of $y(t)$ can both be discrete or continuous.

Given the observation of two states $y_{1}$ and $y_{2}$, the likelihood of the time $\Delta t$ to have elapsed from the first to the second observation is

$$
P\left(\left\{y_{1}, y_{2}\right\} ; \Delta t\right) \propto|\mathbf{C}(\Delta t)|^{-(1 / 2)} \exp \left(-\frac{1}{2}(\mathbf{y}-\mu(\Delta t))^{T} \mathbf{C}(\Delta t)^{-1}(\mathbf{y}-\mu(\Delta t))\right),
$$

where $\mathbf{y}=\left[y_{1}, y_{2}\right]$ is the vector of the two observations, $\mu$ the mean, and $\mathbf{C}$ the covariance matrix of the stochastic process. Both $\mu$ and $\mathbf{C}$ are generally time-dependent and must be estimated form the observations or the analysis of a neural dynamics. In the following, we only consider processes that are constructed such that the time dependency is restricted to the relative time $\Delta t$ between the two events (see below for details).

Given $P\left(\left\{y_{1}, y_{2}\right\} ; \Delta t\right), \Delta t$ can be estimated using a maximum-likelihood (ML) estimator (cf. 2.3.1)

$$
\widehat{\Delta t}_{\mathrm{ML}}=\operatorname{argmax}_{\Delta t} P\left(\left\{y_{1}, y_{2}\right\} ; \Delta t\right) .
$$

The variance of this estimator is bounded from below by the Cramer-Rao bound, which is given by the inverse Fisher information

$$
\operatorname{Var}\left(\widehat{\Delta t}_{\mathrm{ML}}\right) \geq \frac{1}{I_{F}(\Delta t)}
$$

For a Gaussian process, the Fisher information $I_{F}(\Delta t)=\int p(y \mid \Delta t)\left(\partial_{\Delta t} \ln p(y \mid \Delta t)\right)^{2} d y$ is given by

$$
I_{F}(\Delta t)=\frac{\partial \mu}{\partial \Delta t} \mathbf{C}^{-1} \frac{\partial \mu}{\partial \Delta t}+\frac{1}{2} \operatorname{Tr}\left(\mathbf{C}^{-1} \frac{\partial \mathbf{C}}{\partial \Delta t} \mathbf{C}^{-1} \frac{\partial \mathbf{C}}{\partial \Delta t}\right)
$$

The first term of the right-hand side is the information contained in the mean and will be denoted with $I_{F}^{\mu}$ in the following. The second term is the information in the covariance and will be called $I_{F}^{\mathrm{C}}$ 


\subsection{Single Gaussian processes}

We consider a single Gaussian process $y(t)$ of the form given by Eq. 4.1. To incorporate temporal information in the the process, we chose its moments as follows. First, the mean increases linearly in time, $\mu(y)=m t$. This choice complies with the psychophysical observation of a linear relation between physical time and subjective time (cf. Section 2.1.2.1), and is also reflected in several time-dependent neural processes, such as ramping activity (cf. Section 2.2.1.3 and 2.2.3) or propagation of neural activity in synfire chains (cf. Chapter 3).

Second, the variance of the process increases linearly in time, with a finite variance $\sigma_{0}$ at $t=0, \operatorname{Var}(y)=\sigma t+\sigma_{0}$. This simply reflects the linear accumulation of timing errors according to the law of large numbers. And finally, the autocorrelation of process decreases exponentially with the interval between the two points in time that are correlated, $K(y(t), y(t+\Delta t))=$ $\exp \left(-\Delta t^{2} / 2 l^{2}\right)$, where $l^{2}$ is the timescale of the decrease. This form of decrease is typical for many types of stochastic processes [147]. In the following, we abbreviate the correlation $K(y(t), y(t+\Delta t))$ with $K$.

With these assumptions on the moments, we construct the mean vector $\mu$

$$
\mu(\Delta t)=\left(\begin{array}{c}
0 \\
m \Delta t
\end{array}\right)
$$

and covariance matrix $\mathbf{C}$

$$
\mathbf{C}(\Delta t)=\left(\begin{array}{cc}
\sigma_{0}^{2} & \sigma_{0} \sqrt{\sigma^{2} \Delta t+\sigma_{0}^{2}} K \\
\sigma_{0} \sqrt{\sigma^{2} \Delta t+\sigma_{0}^{2}} K & \sigma^{2} \Delta t+\sigma_{0}^{2}
\end{array}\right)
$$

for two observations of the process, the first at time $t=0$ and the second at $t=\Delta t$.

Note that the choice for the time of the first observation is not arbitrary: The fact that some of the moments both change with absolute time $t$ make the process non-stationary. Thus, we assume that the process is reset at time zero. Such a reset is built-in, explicitly or implicitly, in several models of time perception, including the striatal beat model (synchronization of oscillators), models of ramping activity (decrease of firing rates to baseline) and the synfire chain model (ignition of an activity volley), cf. Section 2.2.3 and Chapter 3 . Only when all temporal information is contained in the autocorrelation, the process becomes stationary and the need for a reset is relieved (see below).

Using Eq. 4.4, we can now compute the information contained in the mean

$$
I_{F}^{\mu}(\Delta t)=\frac{\mu^{\prime}(\Delta t)^{2}}{\left(\sigma^{2} \Delta t+\sigma_{0}^{2}\right)\left(1-K(\Delta t)^{2}\right)}
$$


and in the covariance matrix

$$
\begin{aligned}
I_{F}^{C}(\Delta t) & =\frac{\sigma^{4}\left(2-3 K(\Delta t)^{2}+K(\Delta t)^{4}\right)}{4\left(\sigma^{2} \Delta t+\sigma_{0}^{2}\right)^{2}\left(1-K(\Delta t)^{2}\right)^{2}} \\
& +\frac{\sigma^{2} K(\Delta t) K^{\prime}(\Delta t)^{2}}{\left(\sigma^{2} \Delta t+\sigma_{0}^{2}\right)\left(1-K(\Delta t)^{2}\right)} \\
& +\frac{\left(1+K(\Delta t)^{2}\right) K^{\prime}(\Delta t)^{2}}{\left(1-K(\Delta t)^{2}\right)^{2}}
\end{aligned}
$$

To point out the implications of these results, we study three special cases where only the information from one of the moments is available. First, we set $m$ and $\sigma$ to zero, so all temporal information is in the correlation $K$. This is similar, but not identical to the process used by Ahrens and Sahani [5] (see Section 4.4.1). $I_{F}^{\mu}$ is zero in this case, and $I_{F}^{C}$ reduces to the contribution of the third term in Eq. 4.8, so the Cramér-Rao bound is

$$
\mathrm{CRB}_{\mathrm{Cov}}=\frac{\left(1-K(\Delta t)^{2}\right)^{2}}{\left(1+K(\Delta t)^{2}\right) K^{\prime}(\Delta t)^{2}}
$$

which does only depend on $K(\Delta t)$ and its derivative. For large $\Delta t, K$ is much smaller than unity, so this expression can be approximated by $1 / K^{\prime}(\Delta t)^{2}$. The minimal standard deviation of the corresponding estimator then scales like the inverse absolute slope of the correlation function

$$
\sigma_{\widehat{\Delta t}}(\Delta t) \geq \frac{1}{\left|K^{\prime}(\Delta t)\right|}
$$

Exponentially decaying correlations as described by $K(\Delta t)=\exp \left(-\Delta t^{2} / 2 l^{2}\right)$ then yield the minimal standard deviation of the corresponding estimator

$$
\sigma_{\widehat{\Delta t}}(\Delta t) \geq \frac{l^{2}}{\Delta t} \exp \left(\frac{\Delta t^{2}}{2 l^{2}}\right)
$$

Thus, for $\Delta t \ll l$, the minimal timing errors scale like a squared exponential in $\Delta t$.

Next, we study the drift-diffusion process, which we obtain by setting $K=0$, and consider the information in the mean and the variance, captured by $I_{F}^{\mu}$ and $I_{F}^{C}$, respectively. For simplicity, we also set $\sigma_{0}=0$, which does not qualitatively change the results. Then, the minimal errors follow from Eq.4.7 and 4.8, For using the temporal information in the variance,

$$
\sigma_{\widehat{\Delta t}}(\Delta t) \geq \sqrt{2} \Delta t
$$

This corresponds to Weber's law, as the resulting standard deviation is linear in time. On the other hand, if the mean is used as a basis of the time estimate, the minimal variability reads

$$
\sigma_{\widehat{\Delta t}}(\Delta t) \geq \frac{\sigma}{\mu^{\prime}(\Delta t)} \sqrt{\Delta t}
$$

yielding a standard deviation that increases with the square root of time. 


\subsection{Multiple processes}

In the following, we study two models which make use of multiple Gaussian processes with different time scales to see whether the additional information provided by multiple processes changes the scaling behavior of the Cramer-Rao bound.

\subsubsection{The covariance-based model}

The model studies by Ahrens and Sahani [5] is completely described by its covariance structure. The covariance matrix $\boldsymbol{C}_{i}$ of a single Gaussian process $y_{i}$ is given by

$$
\boldsymbol{C}_{i}(\Delta t)=\left(\begin{array}{cc}
\alpha_{i}^{2}+\sigma^{2} & \alpha_{i}^{2} \exp \left(-\Delta t^{2} / 2 l_{i}^{2}\right) \\
\alpha_{i}^{2} \exp \left(-\Delta t^{2} / 2 l_{i}^{2}\right) & \alpha_{i}^{2}+\sigma^{2}
\end{array}\right)
$$

where $\alpha_{i}$ is the weight of each process and $l_{i}$ is its time constant. This covariance matrix slightly differs from the one used in the preceding section, as it also contains an instantaneous variance $\sigma$ which models time-independent variations around to underlying covariance structure. From Eq. 4.4, the Fisher information of process $y_{i}$ then results in

$$
I_{F}^{(i)}(\Delta t)=\frac{2 \alpha_{i}^{4} \Delta t^{2}\left(\alpha_{i}^{4}+\exp \left(\Delta t^{2} / l_{i}^{2}\right)\left(\alpha_{i}^{2}+\sigma^{2}\right)^{2}\right)}{l_{i}^{4}\left(\alpha_{i}^{4}-\exp \left(\Delta t^{2} / l_{i}^{2}\right)\left(\alpha_{i}^{2}+\sigma^{2}\right)^{2}\right)^{2}} .
$$

To estimate a broad range of times $\Delta t$, a maximum-likelihood estimator is used that has access to the joint likelihood of all processes $y_{i}$, and parameters $\alpha$ and $l$ which are assumed to have an explicit functional relation to $i$. A large set of those functions can be summarized by the power-law expressions $\alpha=i^{q}$ and $l=i^{p}$. The total Fisher information is then given by the sum of the individual $I_{F}^{(i)}$, and Ahrens and Sahani studied these expressions and the resulting CRBs by numerical methods. They concluded that summing the information from exponentially decaying correlations with different time constant results in a duration estimate with a standard deviation that follows Weber's law.

Here, we attempt to obtain an analytical expression for the sum of the Fisher information terms. To do so, we approximate the sum with an integral over a continuum of processes with an integration variable $\phi$, and the parameters expressed as $\alpha=\phi^{q}$ and $l=\phi^{p}$. The integration is taken from 0 to the upper limit $\Phi$, which sets the largest timescale of the system to $T=\Phi^{p}$. For a special case, $p=1 / 2$ and $q=0$, the integral can be solved analytically

$$
\begin{aligned}
I_{F}(\Delta t) & =\int_{0}^{\Phi} \frac{2 \Delta t^{2}\left(1+\left(1+\sigma^{2}\right)^{2} \exp \left(\Delta t^{2} / \phi\right)\right)}{\phi^{2}\left(1-\left(1+\sigma^{2}\right)^{2} \exp \left(\Delta t^{2} / \phi\right)\right)^{2}} d \phi \\
& =2 \ln \left(1-\frac{1}{\left(1+\sigma^{2}\right) \exp \left(\Delta t^{2} / T^{2}\right)}\right)+\frac{4}{\left(1+\sigma^{2}\right) \exp \left(\Delta t^{2} / T^{2}\right)-1} .
\end{aligned}
$$

The information only depends on the exponential fraction of $\Delta t^{2}$ and $T^{2}$. As this faction goes to infinity, the information vanishes, whereas for $\exp \left(\Delta t^{2} / T^{2}\right) \searrow \frac{1}{1+\sigma^{2}}$, it approaches infinity. In each case, $I_{F}$ is dominated by the second term. If we approximate $I_{F}$ by this term alone, the minimal standard deviation of the time estimate results in

$$
\sigma_{\widehat{\Delta t}}(\Delta t) \geq \frac{1}{2} \sqrt{\left(1+\sigma^{2}\right) \exp \left(\Delta t^{2} / T^{2}\right)-1}
$$




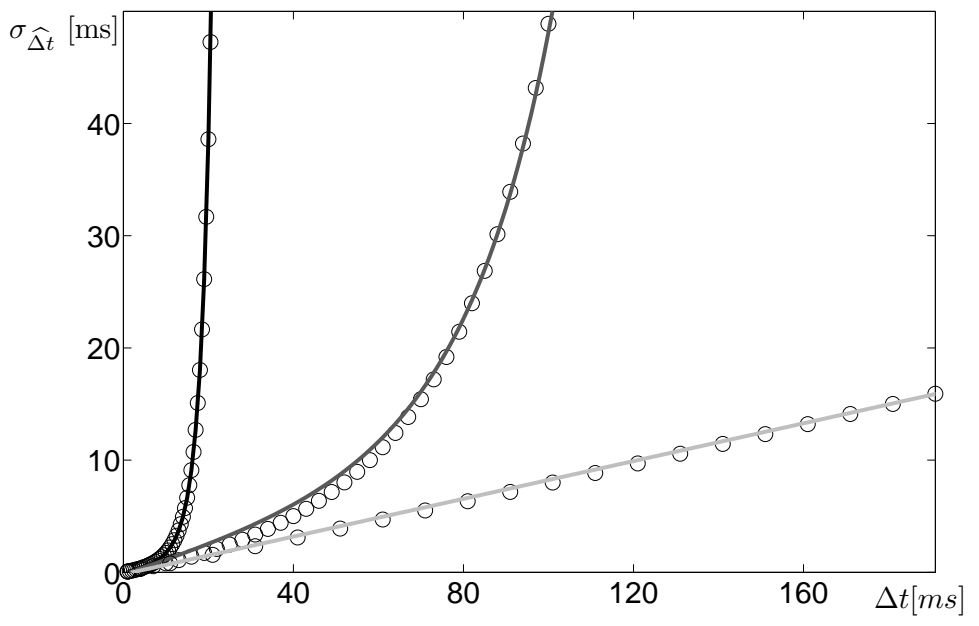

Figure 4.1.: Square roots of the Cramér-Rao bound obtained from integrating the Fisher information (Eq. 4.14) over $\phi$ between zero to $\Phi=50$, with $l_{\phi}=\phi^{p}$ and $\alpha_{\phi}=\phi^{q}$. The exponents were $q=0$ and $p=1 / 2$ (black curve), $p=1$ (dark gray curve) and $p=2$ (light gray curve). In all cases, $\sigma=0.3$. The dots are results from numerical integration and the lines are fits to Eq. 4.17. For $p=1 / 2$ an $p=1$, the exponential deviation from linearity can be seen as soon as $\Delta t$ approaches the maximal time scale $T=\Phi^{p}$.

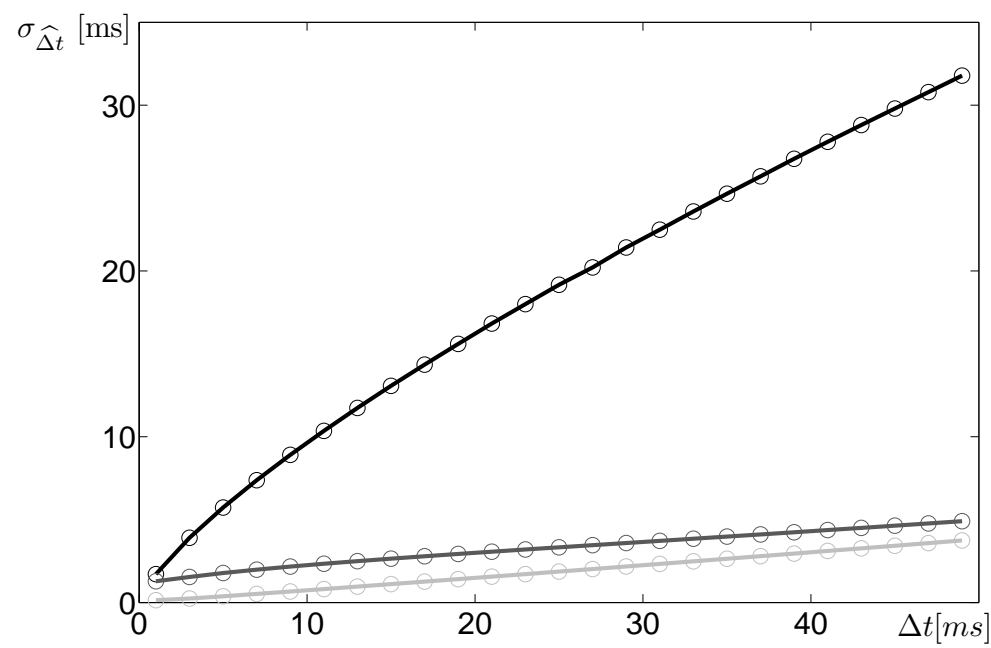

Figure 4.2.: Square roots of the Cramér-Rao bound obtained from integrating the Fisher information (Eq. 4.4) over $\phi$ between zero to $\Phi=50$, with $l_{\phi}=\phi^{p}$ and $\alpha_{\phi}=\phi^{q}$. The exponents are $p=2$ and $q=0$ (black curve), $q=1$ (dark gray curve) and $q=2$ (light gray curve). In all cases, $\sigma=0.3 . \alpha$ affects the offset and inclination of the curves, but only for small $\Delta t$ compared to the maximal time scale $T=\Phi^{p}$. 
Consistent with the analysis for single processes in Sec. 4.3, the minimal standard deviation of the time estimate $\widehat{\Delta t}$ increases like a squared exponential in $\Delta t$, with a timescale that is identical to the largest timescale $T=\Phi^{1 / 2}$ in the system. Thus, only for $\Delta t \ll T$, the increase of the standard deviation can be approximated as being linear.

For other values of $q$ and $p$, our analysis has to resort to numerics. These exponents have been claimed not to change the qualitative behavior of the CRB [5]. However, if an equation of the form (Eq.4.16) holds approximately also for different exponents $p$ and $q$, one would expect that at least $p$ has a major influence on the time scale where the CRB can be considered to increase linearly.

To test this, we integrate Eq. 4.14 numerically for different values of $q$ and $p$ and fitted the resulting minimal timing errors to an equation of the form

$$
A \sqrt{\left(1+\sigma^{2}\right) \exp \left(\Delta t^{2} / T^{2}\right)-1}+B .
$$

The results are shown in Fig. 4.1 for $q=0$ and different $p$, and in Fig. 4.2 for $p=2$ and different values of $q$. The results turn out to be very well matched by Eq. 4.17 for all values of $p$. This implies that it is the time scale $T=\Phi^{p}$ that governs the scaling of the CRB, and not the details of the different integrals. The exponent $q$ which governs $\alpha$, on the other hand, only affects the form of the curves for times that are much shorter than $T$. This limited influence is not surprising, as the behavior of the Fisher information is dominated by the exponential terms and $\alpha$ has only a linear influence.

In summary, the Gaussian process with the largest timescale available determines the range of intervals that can be estimated with a variance approximately obeying Weber's law. For larger times, deviations from linearity are ultimately exponential. Consequently, such large durations are not well estimated by these Gaussian processes but must rely on a different mechanism. This can be shown analytically only for $p=1 / 2$ and $q=0$, but is confirmed numerically also for other values of $p$ and $q$. In particular, the claim that the combination of the information from processes with exponentially decaying correlations results in Weber's law [5] is disproved.

\subsubsection{The synfire chain model}

In Ref. [66], we have presented a neurocomputational model based on multiple synfire chains which is capable of reproducing an experimentally observed error function when it is subject to constrained optimization. Here, we use this model as an example of how a neurocomputational model can be formulated in terms of a stochastic process. Then, we use the present framework to show that the optimal results we obtained are also optimal in this more mathematical sense.

A synfire chain consists of a sequence of layers of neurons $\left\{\mathcal{P}_{i}\right\}, i=1, \ldots, N$ that are connected in a feed-forward manner. It has been shown that neural activity can stably propagate through such a network even in presence of biological noise [70, 37]. So the position of such an activity "volley" within the chain can be used as an estimator of the time $\Delta t$ that has elapsed from the beginning of the propagation. The basic unit of the timer is the transmission time $\tau$ from one pool to the next. Despite the precise transmission, however, a finite temporal jitter $\sigma_{\tau}$ of the activation profile remains, which causes an intrinsic imprecision of the timing results. An estimator of time then takes the form $\widehat{\Delta t}(\Delta t)=\tau j(\Delta t)$. Here, we consider $\tau$ to be constant and $j(\Delta t)$ as a stochastic variable which denotes the index of the layer that 


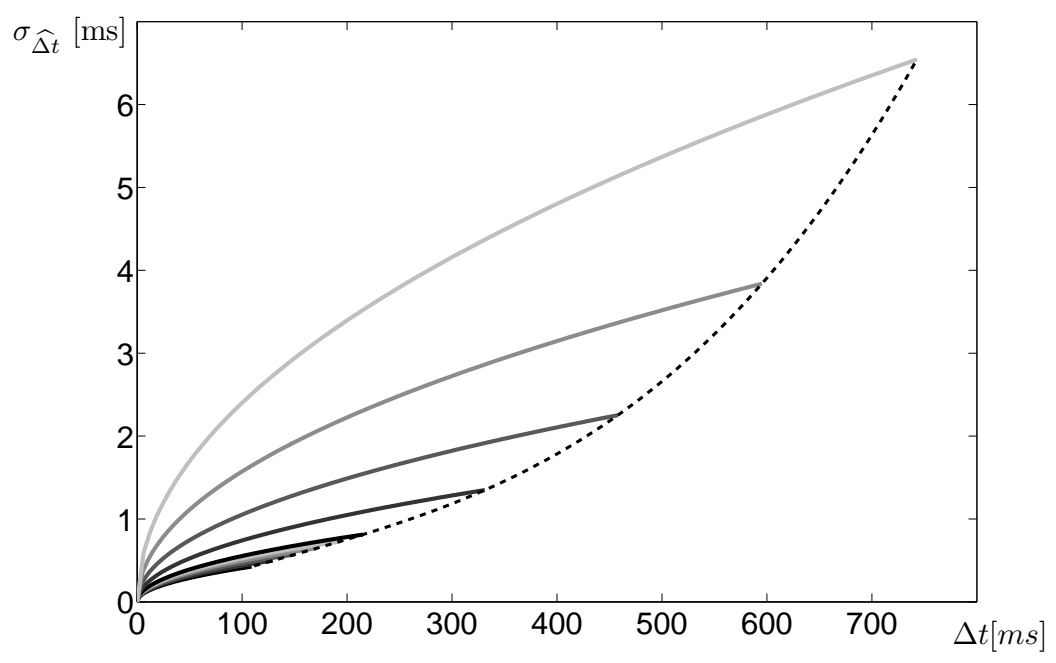

Figure 4.3.: Square-root Cramér-Rao bounds of synfire chains with different mean transmission times $\tau$ obtained as the sum of the information from mean and the full covariance information. The estimation error increases with $\tau$ and the values of $\sigma_{\tau}$ have been taken from Ref. [66. $l$ is determined by simulations of synfire chains to be 0.8 . The dotted line represents the optimal standard deviations for all transmission times (cf. their Eq. 19). This minimal error increases linearly with $\Delta t$ only for a limited range, and super-linearly for large $\Delta t$.

is maximally active at time $\Delta t$. This estimator obviously relies on the mean changes in the stochastic process $j(\Delta t)$, so one can also write the process itself in terms of the estimator

$$
j(\Delta t)=\frac{\widehat{\Delta t}}{\tau} .
$$

The advantage of this formulation lies in the fact that most of the statistics of the estimator $\widehat{\Delta t}$ have already been extracted from the neural dynamics in the previous study, and can thus be directly transferred to $j(\Delta t)$.

Note that the process $j(\Delta t)$ takes only a discrete set of values corresponding to the number of layers of neurons. It can be naturally extended to a continuous-valued process, because the activity at any given time is spread over several layers. Thus, one could include all layers into the process at once and average over layers with weights determined from the number of active neurons $a(j, \Delta t)$ in layer $j$ at time $\Delta t$. The resulting process is given by

$$
s(\Delta t)=\frac{\sum_{k} k a(k, \Delta t)}{\sum_{k} a(k, \Delta t)}
$$

where $a(k, \Delta t)$ is the stochastic variable and $k$ is an index denoting layers. For simplicity, we will restrict ourselves to the discrete process $j(\Delta t)$ in the following.

From the statistical evaluation of the neural dynamics and Eq. 4.18, we can now compute the moments of a single propagation process $j(\Delta t)$. The mean is given by $\mu(\Delta t)=\Delta t / \tau$, 
as $\widehat{\Delta t}$ was shown to be unbiased [66]. Similarly, the variance of the process is $\operatorname{Var}(j)=$ $\operatorname{Var}(\widehat{\Delta t}) / \tau^{2}=\sigma_{\tau}^{2} / \tau^{3}(\Delta t+1)$. Thus, it follows that $\sigma=\sigma_{\tau} / \tau^{(3 / 2)}$. Also, we have introduced $\sigma_{0}^{2}$ as the variance at time zero, which was not considered in the previous study, and we assumed that it takes the same value as the time-dependent variance $\sigma^{2}$. Violation of this assumption would lead to a transient phase where the variance converges to $\sigma_{\tau}$. To deal with this case, one would take the first observation at the first point in time where the transient phase is over. Finally, the correlations of $j(\Delta t)$ decreases exponentially with $\Delta t$, which can be described by the correlation function $K(\Delta t)=\exp \left(-\Delta t^{2} / 2 l^{2}\right)$. Numerical evaluations of the process indicate that the time constant $l$ does depend on the layer, but not on $\tau$. As $l$ does not change much between layers, we used the numerically obtained mean value $l=0.8 \mathrm{~ms}$.

With these specifications, the minimal standard deviation for the estimator $\widehat{\Delta t}$ can be computed from Eqs. 4.13, 4.12 and 4.11. The estimator introduced above is mean-based, thus its minimal standard deviation (cf. Eq. 4.13) is

$$
\sigma_{\widehat{\Delta t}}(\Delta t) \geq \tau \sigma \sqrt{\Delta t+1}=\sigma_{\tau} \sqrt{\frac{\Delta t+1}{\tau}} .
$$

Up to the term from $\sigma_{0}$, this is exactly the scaling of the error that was found to be optimal [66], so the estimator $\widehat{\Delta t}$ used there was indeed optimal in terms of information-theory. Furthermore, one could also try to exploit the additional information in the variance and the correlation by using e.g. a maximum-likelihood estimator (cf. Eq. 4.2). To judge how much additional information one could gain in this way, we examine the values of the parameters of the synfire process. From the numerical evaluations in the previous study, the range of values for $\sigma$ and $\mu=1 / \tau$ can be determined. Within a range of parameters where the propagation of activity within the synfire chain is stable, $\tau$ ranges from 0.5 to $5.5 \mathrm{~ms}$ and $\sigma$ takes values between 0.03 and $0.45 \mathrm{~ms}$. Thus, $\sigma / \mu$, the scaling factor of the increase of the timing error, lies between 0.042 and $0.19 \mathrm{~ms}$. Both of these values are much less than $\sqrt{2}$, the scaling factor of the minimal error from the variance. So it can be expected that the variance will not add much information (cf. Sec. 3.4). Similarly, the time scale of the correlation, $l=0.8 \mathrm{~ms}$, is extremely short compared to the interval durations of a few hundreds of milliseconds. Thus, the information from the covariance will also be small. These theoretical considerations are confirmed when plotting the minimal standard deviations from a number of processes together based on all available information sources, with the same parameters as used in Ref. 66] (their Fig. 5). Indeed, this figure is virtually identical to the one which is only based on the mean.

\subsection{Discussion}

We have presented a statistical framework for optimal estimation of time based on the first and second moments of a stochastic process and compared the contributions of temporal information in the mean, the variance and the covariance. Of course, the combination of all these sources of information always yields best results. However, given a forced choice between different contributions, relying on the mean evolution of the process gives best results, in the sense that the minimal timing error scales with $\sqrt{\Delta t}$. This is the approach taken by most of the present models of temporal processing,. If the process has zero mean, or a mean that is too much obscured by noise to be reliably read out, the second-best choice would be the variance, 
which yields and increase of errors linear in $\Delta t$. This would be possible whenever there is a sufficient amount of independent noise in the system. Relying solely on the covariance, on the other hand, is less favorable because of the exponential increase of timing errors. As such a scaling is not seen in any psychophysical experiments, it can be concluded that either this mechanism is not used in the brain, or the time scales of the covariance decay are very long. A more recent model [25] places itself somewhat in between deterministic and stochastic approaches and may actually exploit both sources of information. It relies on slow processes of the single neuron level such as short-term synaptic plasticity, but also on a broad distributions of parameters and the joint, state-dependent response of a whole network (cf. Section 2.2.3). It would be interesting to assess the statistical properties of this model and analyze it stochastic terms. More generally, the framework we presented here is suitable to test arbitrary neural models according to their compliance with psychophysical results. Of course, this is only true under the assumption that the brain computes an estimate of time that is optimal in the Bayesian sense.

Given the favorable property of the information in the mean of the process that it results in a $\sqrt{\Delta t}$ scaling of errors, one may ask why there is a need for any other source of information. In the case of a synfire chain, the optimal choice appears to be a single chain containing a very large number of pools and very fast transmission. There are several arguments against such a construction. The most obvious one is the massive parallel computing architecture of the brain, as opposed to a single, very fast CPU that is used in a computer. The advantage of parallel evaluation of multiple computations (here: chains) are twofold: On the one hand, it allows for parallel computation of time and various other sensory information. On the other hand, many short chains allow for a much larger robustness against catastrophic, i.e. the failure of neural transmission in a given pool. In a single large system, the probability of such a failure increases with the number of pools, and can not be compensated. Both of these problems are resolved by many parallel chains.

Another argument especially for parallel chains with different time constants may lie in the a priori information about the common distribution of time intervals. For instance, a prior for the distribution of time scales such as

$$
P(\Delta t)=\beta \exp (-\beta \Delta t) \Theta(\Delta t),
$$

with $\beta \ll 1$, can be assumed to assert an over-linear excess of small time intervals. Suppression of errors on these small time scales is achieved precisely by using a large number of chains that are capable to represent these times (cf. Fig. 4.3). Finally, the underlying structures are also subject to certain limitations with respect to their evolvability [71] (see Section 3.6] for further discussion of this issue).

The parallel use of multiple Gaussian processes with different time scales does not qualitatively change the scaling behavior of the timing errors. However, information from multiple processes can be combined to reduce the total error or to extend the range of intervals that can be encoded.

In summary, although specialized structures may not be needed for temporal processing, best estimation results are obtained when there is process that changes with time in the mean. 
However, this may also be a process that carries out some other computation in parallel, so the idea of a distributed, non-specific perception of time [28] is not contradicted by our results. On the other hand, noise can even enhance the precision of the estimate if it contains a temporal structure itself that can be decoded and that has a reasonable magnitude that does not blur out the information in the mean. This phenomenon is know as stochastic resonance [136]. To establish such an optimal balance of noise may be a goal for self-organization in the brain. 


\section{Cross-modal interaction of time perception and motor timing}

As we discussed in Chapter 1 and 2, it is currently debated whether the perception of time relies on a centralized internal clock or on modality- and task-specific mechanisms. In this chapter, we describe four psychophysical experiments comprising a time perception task and a concurrently performed motor task with visual feedback. Time appeared as longer when visually observed movement was faster. The execution of the tracking motion did not contribute to this effect, but impaired discrimination performance by dual-task interference. This study demonstrates direct integration of temporal information from different modalities and provides causal support for the notion that time perception and continuous motor timing rely on separate mechanisms. The results are consistent with Bayesian integration of modalityspecific temporal information into a centralized "temporal hub", which may be subject to attentional modulation.

\subsection{Introduction}

Time is a perceptual quantity that abstracts from sensory modality. Humans can assess the temporal dimension of visual, auditory and multidimensional stimuli such as motion, speech, or music. Similarly, any directed motor act relies on precisely timed coordination of a large set of muscles. Yet, it is unclear how temporal information is integrated in all these situations. Early proposals of a centralized clock that governs all temporal computations [33, 55] have been challenged by recent findings of modality-specific timing processes, e.g. in early vision 84, 135, and it is also a nontrivial task to disentangle the integrated perception of time from low-level sensory processes when being considered within a single modality [80].

A suitable way to resolve this issue is to study cross-modal distortions of time perception. Subjective duration is easily manipulated by a wide range of non-temporal factors, some of which are modality-specific [42]. For instance, moving visual stimuli have been shown to be judged as longer in duration compared to static ones [20, 19], and this effect increases as the velocity of the moving stimuli gets faster. Thus, if a manipulation of a stimulus feature in one modality results in the distortion of subjective duration in another modality, this provides evidence that time perception is closely interlinked between these two modalities. In the same way, one can assess the connections between time perception and motor timing by manipulating temporal characteristics of a motor task and looking for resulting distortions in a concurrently performed time perception task.

Existing studies that employed the distortion approach rely on stimulus factors that are related to attentional resources [176, 29]. Subjective duration is directly affected by the 
allocation of these resources [22, 21], so it may be that the observed distortions are the product of changes in global attention rather than direct interaction between modalities. These possible confounds currently defy a straightforward account for cross-modal interactions in time perception, as well as inconsistencies in the results (see below). Furthermore, the approach of cross-modal distortion has not been applied yet in linking time perception with motor timing. Results that link those two domains are currently restricted to the analysis of correlations in measures of performance for time perception and motor timing tasks [93, 78, 155, 189.

We assessed causal links between auditory time perception of durations of about $100 \mathrm{~ms}$, and both continuous motor timing and a comparable visual tracking task. We combined a visually guided motor task with a concurrently performed auditory time perception task, within series of four experiment:1. Participants were required to perform an arm movement to follow an elliptic trajectory prescribed by a moving target on a screen. At specific segments of the ellipse, two auditory stimuli were presented in sequence and should be discriminated according to their duration. By manipulating speed and curvature of the target motion during the presentation of each interval, we studied whether subjective duration (point of subjective equality, PSE) and discrimination performance (difference limen, DL) was influenced by changes in these motion parameters, and whether this influence is caused by visual observation or actual performance of the motion. Each participant also performed two single-task experiments, in which either the motion or the time perception task was performed alone.

In Experiment 1, we configured the target motion such that the tangential velocity and the curvature of the target motion followed a power law with an exponent of $-1 / 3$. This relation is naturally fulfilled in voluntary continuous motion [179]. Because of this manipulation, the movement differed both in curvature and in angular velocity at the times where the first and the second tone was presented. The first auditory stimulus was given at one of the apices of the ellipse, and the second one at the subsequent apex. This yields four conditions for stimulus presentation, two of which are identical regarding curvature and velocity. We averaged all performance measures over those identical states and considered only differences between conditions where the motion is more curved at the second tone compared to the first one (called curves) and the conditions where this order is reversed (straights)2.

\subsection{Results}

If the duration distortion induced by visual motion [20, 19] carries over to the auditory domain, one would expect the PSE to decrease from straights to curves. This was indeed the case (Fig. 5.1, $t(19)=-2.35, P<0.030$, Cohen's $d=0.31$ ). We confirmed that participants

\footnotetext{
${ }^{1}$ See Section A.1 in the appendix of the thesis for more details on methods and materials

${ }^{2}$ To ensure that the observed effects are really due to differences between straights and curves and not to differences of the individual positions, we performed a control experiment in with we rotated the ellipse by 90 degrees, so that the curves were at the top and bottom of the screen, and the straights to the left and the right. The results found in Experiment 1 were reproduced. For more details, see supplemental text in Section A.2 in the appendix (Experiment 1b).
} 


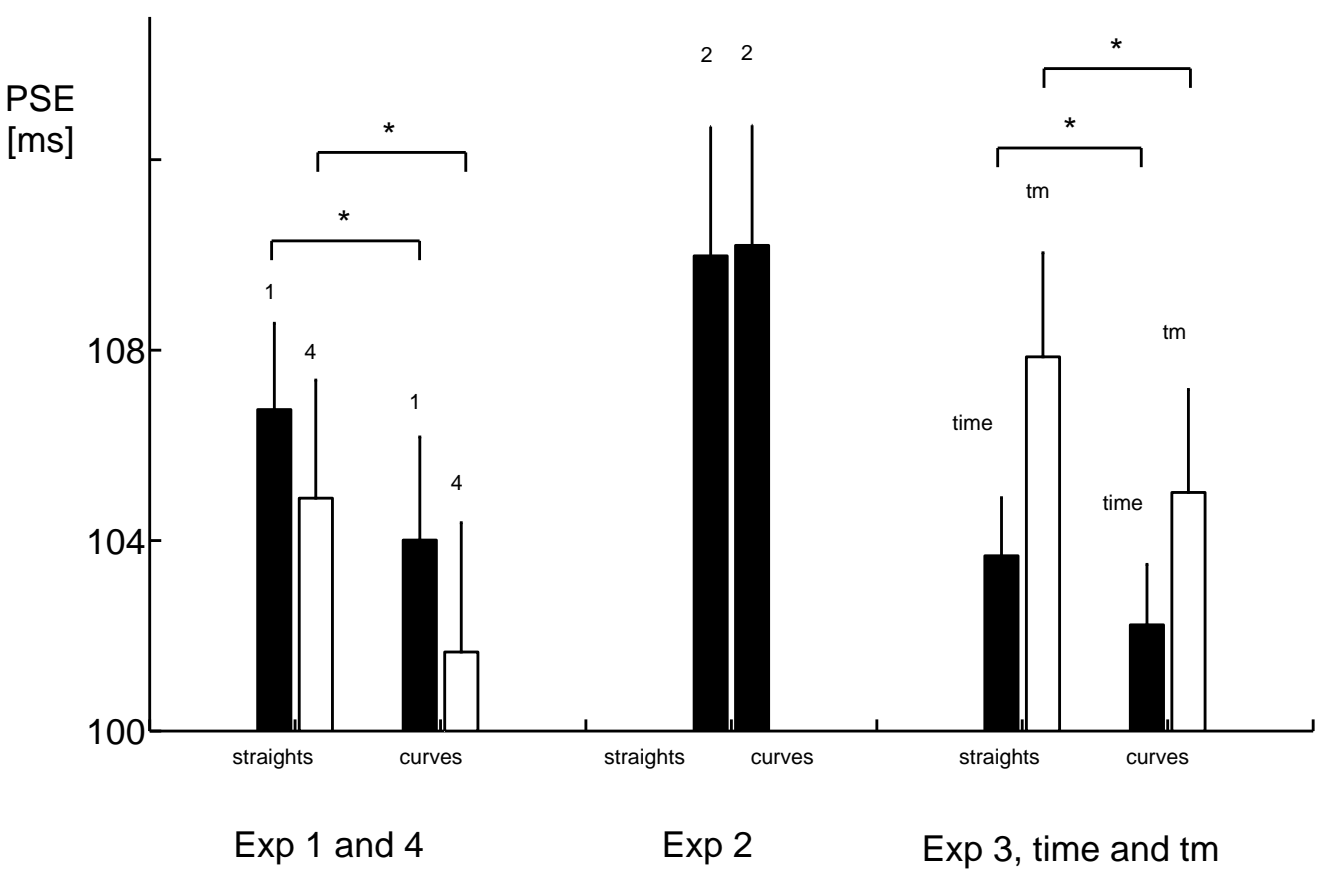

Figure 5.1.: Subjective duration (PSE) for the straights and curves condition, respectively, in Experiment 1 to 4 . Error bars are standard errors; brackets with stars depict significant differences $\left({ }^{*} P<0.05\right)$. In Experiment 1,3 and 4 , the PSE is significantly lower in curves compared to straights, indicating that durations were perceived as longer at the upper and lower straight compared to the left and right curve. In Experiment 2, there was no such effect.

actually followed the trajectory we prescribed by measuring the motion parameters of the actual motion (see methods in Section A.1). As expected, curvature increased $(t(39)=94.7$, $P<0.001$, Cohen's $d=20.3)$ and velocity decreased $(t(39)=-74.1, P<0.001$, Cohen's $d=25.1$ ) from straights to curves (Fig. A.3). The relation between curvature and tangential velocity could be well fitted to a power law with a mean exponent of -0.279 (SD 0.024), which is close to $-1 / 3$.

From Experiment 1 alone, it is hard to determine the cause of the distortion in subjective duration, as both velocity and curvature varied between conditions, and the PSE was correlated with both of them (see supporting text in Section A.2). In Experiment 2, we kept the tangential velocity constant along the trajectory, so only curvature changed between conditions. Straights and curves no longed showed a PSE difference (Fig. 5.1, $t(19)=0.37, P=0.72$, Cohen's $d=0.02)$. However, participants did not accurately follow the motion of the target. Tangential velocity still decreased from straights to curves $(t(39)=-22.7, P<0.001$, Cohen's 
$d=6.2)$, while curvature increased from straights to curves $(t(39)=40.2, P<0.001$, Cohen's $d=14.0$ ), as expected (Fig. A.3). The relation between curvature and tangential velocity was again well fitted by a power law, although its exponent now deviated strongly from $-1 / 3$ (mean -0.144, SD 0.059). It is frequently reported that people tend to stick to the $-1 / 3$ power law relation [179], even when instructed otherwise [180, 178]. Our results are consistent with a strategy where participants compromised between the demands of the instructions and the internal constraints of the movement.

The key difference between Experiment 1 and 2 was in the speed of the observed target motion. Experiment 3 directly compares the effects of motion that is perceived and motion that is actually performed. We followed the same protocol as in Experiment 1, but changed the single-task time perception experiment such that it comprised the same four conditions as in the dual-task experiment. Here, the conditions were defined according to the position of the target (see methods in Section A.1). The PSE decreased from straights to curves both in the single task $(t(19)=-2.40, P<0.027$, Cohen's $d=0.27)$ and the dual task experiment $(t(19)=-3.16, P<0.005$, Cohen's $d=0.30)$ with comparable effect size (Fig. 5.1). Furthermore, there was no significant difference of the distortion effects between the single- and dual-task condition $(F(1,19)=1.3, P=0.26)$, neither were there any significant differences between the motion parameters in Experiment 1 and 3 (data not shown). Collectively, Experiment 1-3 show that duration distortion is caused by the speed of the perceived motion, and that performed motion does not contribute to this effect.

Could the distortion reflect a change in global attention rather than a direct multi-modal interaction? To test this, we first compared both DL and PSE for the dual-task experiments 1-3 and their respective single-task experiments where only the time perception task was performed. Both a decrease in discrimination performance (increased DL, Fig. 5.2, $F(1,19)=$ 43.5, $P<0.001, \eta_{p}^{2}=0.70$ ) and in subjective duration (PSE, Fig. 5.2, $F(1,19)=10.1$, $\left.P<0.005, \eta_{p}^{2}=0.35\right)$ were present in the dual task condition in Experiment 1-33, which are both signs of attentional interference [22]. To our best knowledge, this is the first report of dual task interference in the milliseconds range induced by a motor task.

To show that the duration distortion effect is independent from this dual-task interference, we conducted Experiment 4, where we included an extended training of the motor task prior to the actual experiments. Training of the secondary task has been shown to diminish dual-task interference on time perception [23. Participants practiced the motor task until they reached a defined target deviation averaged over two minutes (see methods in Section A.1). Consistent with former studies, neither the DL difference (Fig. 5.2, $t(19)=1.69, P=0.11$ ) nor the PSE difference (Fig. 5.2, $t(19)=1.03, P=0.31$ ) between single- and dual-task conditions was significant any more. On the other hand, the PSE difference between straights and curves remained (Fig. 5.1, $t(19)=-2.39, P<0.027$, Cohen's $d=0.28$ ). As additional evidence for independence of duration from attentional resources, note that the effect sizes were the

\footnotetext{
${ }^{3}$ These effects were also significant for DL when experiments were analyzed individually, whereas the PSE only showed trends of differences for Experiment 2 and 3.
} 


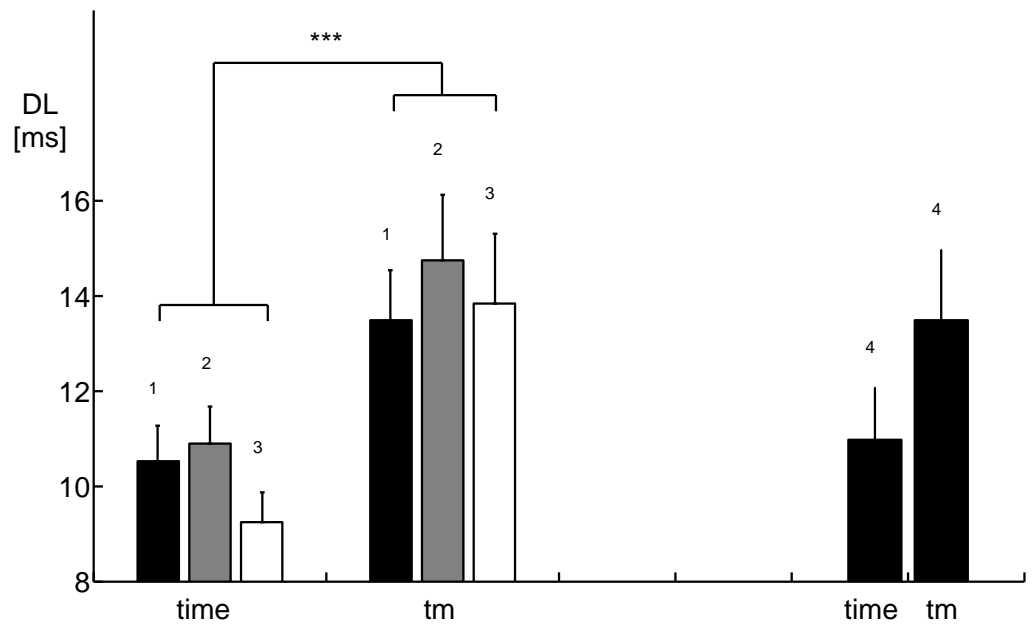

Exp 1 to 3

$\operatorname{Exp} 4$

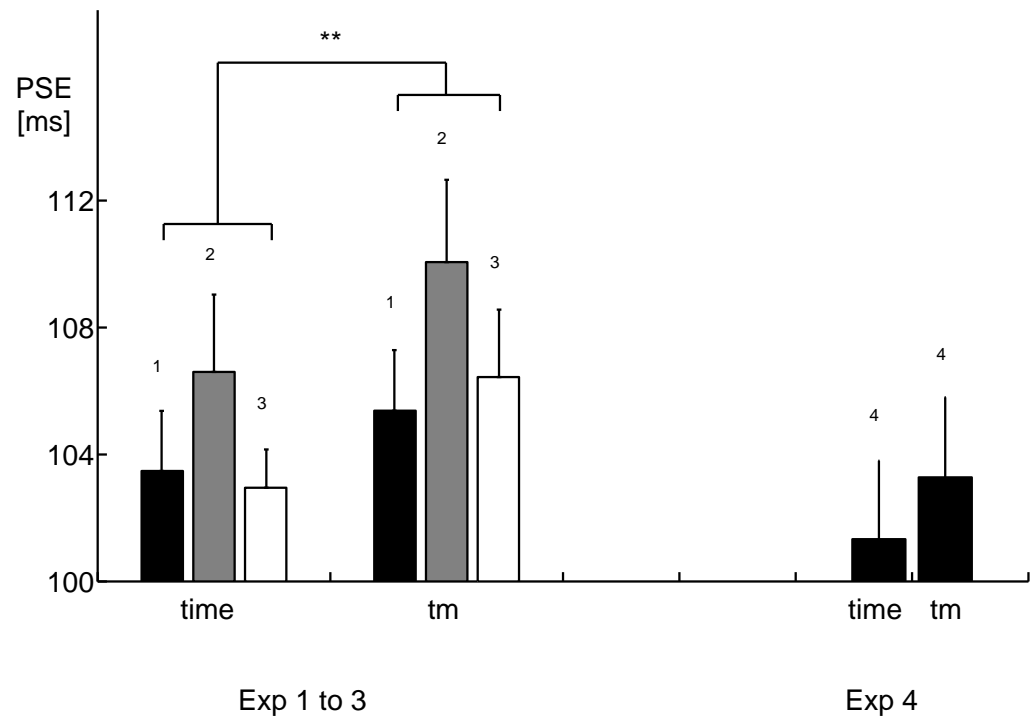

Figure 5.2.: Discrimination performance (DL) and subjective duration (PSE) compared for single-task (time task only) and dual-task experiments in Experiment 1 to 4 . Error bars are standard errors; brackets with stars depict significant differences $\left({ }^{* *} P<0.01,{ }^{* * *} P<0.001\right)$. Both DL and PSE increase from single- to dual-task in Experiments 1 to 3, but not in Experiment 4. 
same for the single- and dual-task condition in Experiment 3, and that the DL did not change between straights and curves consistently for all experiments $(t(79)=0.96, P<0.34)$.

\subsection{Discussion}

In three out of four experiments (Experiment 1,3 and 4), auditory time perception was consistently distorted by visually observed motion (Fig. 5.1). This distortion depends on the speed of the observed motion - intervals are perceived as longer when motion is faster, and this effect vanishes when observed motion speed is constant (Experiment 2), but is still present when participants did not perform active motion (Experiment 3). These results indicate that the well-documented duration distortion induced by visual motion speed [20, 19] carries over to the auditory domain. Unlike former studies [176, 29, we controlled for attentional factors by comparing the distortion effect with dual-task interference [22]. Distortion was the same in the single- and dual-task experiment (Experiment 3) and the distortion effect was not diminished when the motor task was subject to additional training (Experiment 4), in contrast to interference effects on discrimination performance. From these results, we conclude that duration distortion is not based on cognitive resources. The fact that we did find dual-task interference for intervals in the range of $100 \mathrm{~ms}$ contributes to the emerging view that attentional factors affect temporal processing for intervals both above and below $1 \mathrm{sec}$ [151], challenging the notion of distinct mechanisms for time perception in these two domains [150, 110].

We did not find evidence that active performance of motion contributed to duration distortion. Performing the motor task did not strengthen the effect compared to purely observed motion (Experiment 3), and when visual motion speed was constant, changes in the speed of the actually performed motion did not induce any distortion (Experiment 2). These results suggest a largely separated set of mechanisms for the timing of continuous motor acts and the perception of time. Former studies that made the same proposal only reported lack of correlations between perception and continuous motor timing [155, 189]. Our results are consistent with the distinction between "emergent" and "event" timing which governs continuous motor timing and both time perception and discrete motor acts, respectively [190].

Regarding the nature of the explicit representations of time, we propose that the multimodal interaction comes about by integration of temporal information in a "temporal hub", which is consistent with the recent notion of Bayesian integration of general multi-sensory information [34]. Within this framework, a stimulus is perceived independently in different modalities and then integrated into a single percept. The magnitude of the contribution from each modality is weighted by the information or reliability of the respective channel which may vary in different situations. Taking this view on time perception [176], temporal information is gathered simultaneously in all modalities available, possibly as a by-product of other sensory computations [66, and then converges into a central brain site where an integrated judgment about the temporal stimulus is generated according to the relative reliability of each modality. A possible anatomical location for this temporal hub could be the basal ganglia, where many cortical nerve fibers converge and which has been discussed previously as a brain site to orchestrate temporal information [24]. Within this framework, several phenomena of time 
perception can be readily explained: Dual-task interference with time perception [21, 22] corresponds to an impaired transmission of the temporal information to the hub, caused by decreased reliability of the temporal signal due to diminished attention. The conflicting results according asymmetries in cross-modal interaction [176, 29] are reflections of different degrees of salience and reliability in the visual and auditory modality, owing to difference in the nature of the stimuli used in both studies (static stimuli [29] vs. dynamically changing stimuli [176]). And finally, observed beneficial or distorting effects of congruent or incongruent visual and auditory rhythms, respectively [64] can be seen as the result of an average of temporal stimuli from both domains, which decreases variability when information from both channels is the same, but increases it when the information is conflicting. Such averaging effects have been studied before in purely auditory sequences [67, 39].

In summary, our results demonstrate a direct interaction between visual and auditory time perception, while no connection between the timing of continuous motor acts and the perception of time can be found. The cross-modal interaction in the perceptual domain is consistent with Bayesian integration of temporal information from different sources into a temporal hub, according to the relative reliability of these sources. This kind of integration may be the brains' solution to the problem of constructing unique and reliable representations of time despite of the fact that duration could be distorted by a large number of non-temporal stimulus features in each individual modality. 


\section{Cognitive aspects of time perception in temporal sequences}

In this chapter, we extend our research from single intervals to sequences of intervals. Combining experiments and modeling, we study how the discrimination of time intervals depends both on the interval duration and on contextual stimuli. Participants judged the temporal regularity of a sequence of standard intervals that contained a deviant interval. We find that the performance to detect the deviant increases with the number of standards preceding the deviant and decreases with the duration of the standard. While the effect of the standard duration can be explained by an neural network model that realizes the concept of multiple synfire chains (cf. Chapter 3), the position effect is incorporated into the model by an in-situ averaging process. Furthermore, experiments are discussed that are critical for the predictions of the model. In conclusion, the model shows that sequence processing may rely on the same mechanisms as the processing of individual intervals.

A slightly modified version of this chapter has be published in the proceedings of the Neural Computation and Psychology Workshop 2008 (NCPW11) 67]. We gratefully acknowledge the work of our collaborator Stefan Blaschke, who designed and performed the experiments and provided the original idea of the adaptive memory system. Our contribution to this chapter mainly lies in the formalization of this model, and in contributions to the conception of the experimental paradigm.

\subsection{Introduction}

Whenever we listen to somebody talking, or to a piece of music, we are presented with a sequence of stimuli that contain information in their duration and timing. For instance, the phonemes / ba/ and / pa/ differ by only 25 to $50 \mathrm{~ms}$ in their onset time but can still be reliably discriminated. This discrimination is even better when the phonemes are embedded in a sequence that forms natural speech.

While speech is a quite complex example of a sequence including semantic information, the neural mechanisms that enable discrimination of interval durations are not well understood even for much simpler sequences with purely temporal context, or even single intervals. Despite of numerous experimental and theoretical studies on the topic [61, 58, 149], many ambiguities even about the psychophysical regularities remain. For instance, it is established that variability of time estimates $\sigma_{T}$ increases as the intervals $T$ to be estimated get longer [61], but it is debated whether this increase is linear in $T$ (Weber's law) [55], even steeper [15] or less steep than linear 54. Similarly, for the question of whether context information enhance discrimination performance, there is both supporting [39, 78] and contradicting evidence [164, 141]. 
We approach these two questions with an experimental paradigm (Sec. 6.2) where participants discriminate the duration of a variable interval from the constant standard durations of a number of previously presented intervals. The more standards are presented before the variable interval, the more context information is available. Varying the standard duration between blocks, we can simultaneously assess the decrease of discrimination performance with the interval duration. This experiment can be seen as a critical test between two classes of models: Static models like the classical pacemaker-accumulator system [33] predict no context effects at all, while dynamic models such as the multiple look model [39] predict an improved performance with increasing context information. Our results support the latter class, as performance increases at later positions of the variable interval.

In Sec. 6.3, we formalize the concept of the multiple look model [39] that improved performance results from averaging previous temporal information to reduce discrimination errors. The model provides a statistical framework for perception of both single intervals and sequences of intervals, as judgments about sequences are based on comparison of the individual intervals it is composed of. The model can be readily extended to account for more complex aspects of time perception and has partly been implemented as a biological neural network [66]. This implementation is based on general connection principles in the neocortex and does not depend on any modality-specific properties. Finally, Sec. 6.4 discusses the results and gives an outlook on further experiments.

\subsection{Sequence experiment}

\subsubsection{Method}

23 psychology undergraduates (mean age 23 years, 17 female) participated in the experiment for partial fulfillment of course requirements. They were naive to the purpose of the experiment, but were debriefed afterwards, including feedback about their performance. In each trial, a sequence of seven intervals filled with white noise was presented via headphones. Six of these intervals were standard intervals (STI) with a constant duration, while the seventh was a variable interval (VTI). All intervals were separated by an inter-stimulus interval (ISI) with a duration identical to the STI. Participants were instructed that a deviating interval could be presented at any of the seven positions in the sequence and that if there was a deviating interval in the sequence, it would be the only one. The task was to decide whether the presented sequence was regular or irregular. As independent variables, we used the position of the VTI within the sequence (position 1 to position 7), which was randomized from trial to trial, and three different STI durations $(50 \mathrm{~ms}, 150 \mathrm{~ms}$ and $250 \mathrm{~ms}$ ), which were tested in separate blocks. The duration of the VTI was adjusted by a weighted up-down method [86]. Starting from an initial value, the duration was increased (step-up) if the participant had judged the sequence as "regular" and decreased (step-down) after an "irregular" judgment. The adjustments were done independently for each position of the variable interval. We chose the step sizes such that the VTI converged to the .75 percentile of the answer "irregular". As the dependent variable, we used the $75 \%$ detection threshold $V_{75}$, which can be computed from the percentile by subtracting the respective standard duration. The smaller the threshold, the better is the performance in detecting a deviant interval. 

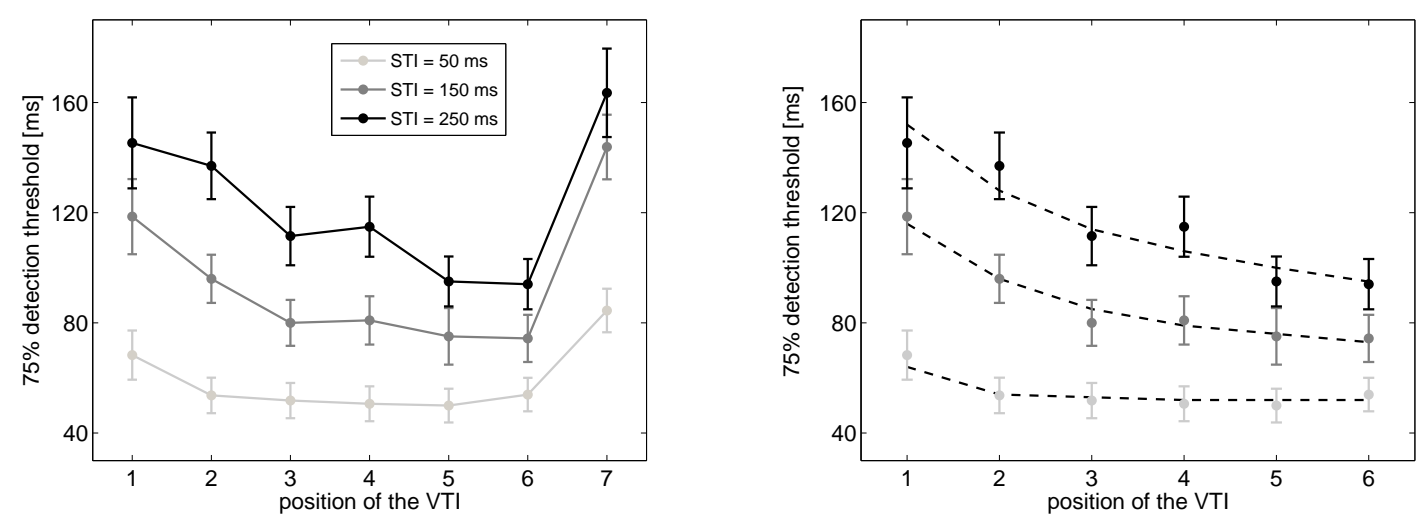

Figure 6.1.: $75 \%$ detection thresholds as a function of the position of the variable interval for three standard durations. The dots are means over participants with standard error bars. (Left) Data for all seven positions. (Right) Data for position one to six. The lines are fits of this data to Eq. 6.6 (see Sec. 6.3.2.4). The color map is the same in both figures.

\subsubsection{Results}

Fig. 6.1 shows the mean values of $V_{75}$ as a function of both the position of deviant and the duration of the STI. Three effects are apparent: The threshold increases with the standard duration, decreases from position one to six, and finally, increases again at the last position. To confirm these effects statistically, we performed a two-way ANOVA with the factors position and standard duration (levels as indicated above). The ANOVA showed highly significant effects for both factors, $F(6,132)=35.61, p<.001, \eta=0.62$ and $F(2,44)=68.97, p<.001$, $\eta=0.76$, respectively, and also an interaction, $F(12,264)=8.02, p<.001, \eta=.27$ ( $\eta$ is short for partial eta-squared). These results did not qualitatively change when the seventh position was excluded from the analysis (data not shown).

To further analyze the increase of $V_{75}$ with the STI duration, we take the mean over all seven positions within an STI duration and calculate the Weber fraction $\bar{V}_{75} / S T I$ for each STI duration. The values were 1.18, 0.64 and 0.49 for $S=50 \mathrm{~ms}, 150 \mathrm{~ms}$ and $250 \mathrm{~ms}$, respectively. Decreasing Weber fractions are in accordance with standard theories of temporal perception 61] within this range of relatively short durations.

The significant decrease of the detection threshold from position one to six established that the number of STIs presented before a VTI indeed improves the performance to detect the deviant. This rules out static models of time perception [33 that would predict no such effect. However, also adaptive models that predict improved performance with increasing number of standards do not predict the decrease in performance at the final position. 


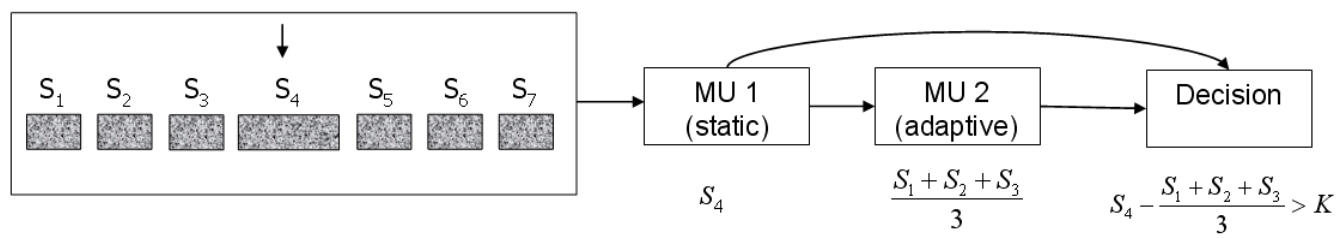

Figure 6.2.: Illustration of the model structure.

\subsection{Serial memory model}

We now develop a model that aims to explain the findings in the present experiment. The model composes the representation of a temporal sequence from the representations of the individual intervals. The basic structure of this model (Fig. 6.2) is similar to the classical pacemaker-accumulator system [33, although its elements include mechanisms of adaptivity. Each interval is first encoded in a single-interval representation. We proposed a neural model for this encoding [66], which we briefly present in the next section.

The second stage in the model is a memory system with two units (MU). These units also exist in the original model, but we make two modifications. First, the units are arranged in serial, e.g. the representation of interval one is first stored in MU1, but as the second interval is encoded, interval one is shifted to MU2 and interval two is stored in MU1 and so on (cf. Fig. 6.2). And second, while MU1 always contains a representation of the individual intervals, in MU2 the representations of all presented intervals are averaged to decrease variability.

Finally, in the third stage, the intervals represented in the two units are compared, and whenever the difference between the two exceeds a certain criterion, a deviant interval is detected. In this respect, the framework is similar to classical signal detection theory.

\subsubsection{Single interval representations by synfire chains}

A neural correlate of an interval representation should consist of a neural network that is able to store a wide range of time intervals with high precision. A neural structure that fulfills these requirements is the synfire chain [2], a layered network of spiking neurons with feed-forward connectivity. This type of network has been shown to enable stable propagation of neuronal activity: If a sufficient number of neurons in the first layer is activated, neurons in the second layer also start spiking after some time, and this activation in turn is transmitted to the third pool, and so on. It has been shown that under broad conditions on the strength and timing of the initial activation [70, 37] and the model parameters [66], this propagation is stable, and activity travels along the layers like a wave. The propagation is linear in time and the temporal spread $\sigma_{L}$ of the wave at each layer converges to a constant fixed point value in the range of milliseconds [70, 37, even in the presence of synaptic background noise. Therefore, 


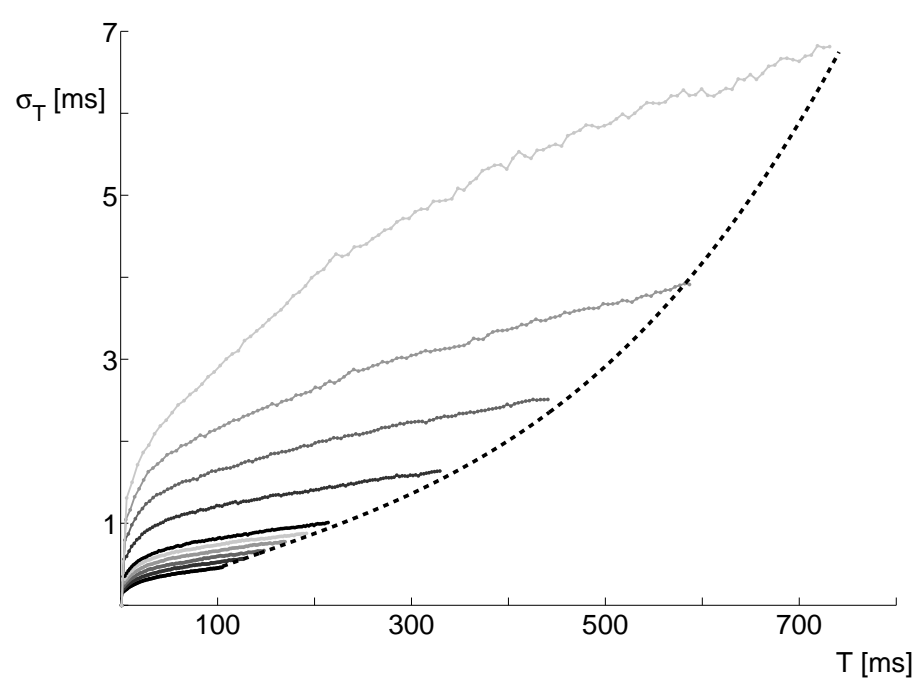

Figure 6.3.: Timing error, e.g. standard deviation $\sigma_{T}$ of the total runtime of an activity wave as a function of time $T$ for various transmission speeds of the chains. The solid curves depict simulation data and the dotted line represents the optimal timing error $\sigma_{T}^{*}(T)$ from Eq. 6.1, It is close to the lower envelope of the simulation data.

the system is able to translate temporal information into a precise quasi-spatial code: The time elapsed since the initiation of the wave is represented in the position of the layer that is currently most active [66].

Variability in the representation arises from the remaining temporal spread $\sigma_{L}$ in the spikes. This constant error in each layer accumulates to smear the arrival time $T$ of the wave at layer $i$ to a standard deviation $\sigma_{T}$ proportional to the square root of $i$. Therefore, the Weber fraction $\sigma_{T} / T$ decreases with the interval length like $1 / \sqrt{T}$, consistent with the results of our experiment.

For short intervals up to a few hundred milliseconds, this result of a decreasing Weber fraction has also been found previous experiments [54, 61]. However, the steeper increase found at longer intervals (linear or even superlinear with duration [61, 149, 55, 15]) is not easily reconciled with the accumulation of neuronal noise. For the steeper increase at longer intervals, there must be an additional constraint. In a synfire chain, the most obvious of such constraints is a finite chain length $L$. With a given mean transmission delay $\Delta t$ from one pool to the next, the maximal interval to be represented is $T=\Delta t \cdot L$. For longer intervals, a chain with a higher value of $\Delta t$ must be used. We could show that the speed of the activity wave can be manipulated by various model parameters [66, but that any change in the synfire model that increases $\Delta t$ also increases the spread of the spike times $\sigma_{L}$ and thus, results in a larger timing error $\sigma_{T}$ (Fig. 6.3) 66]. From Fig. 6.3, it is also apparent that there exists an optimal chain for each interval of time to be encoded, meaning that the timing error $\sigma_{T}$ 
is minimal. As the increase of this error with $\Delta t$ is much larger (order 3 ) than the increase along the layers (order 1/2), it is always optimal to use the entire length of the chain with the lowest $\Delta T$ that is able to encode the current interval. The form of the optimal timing error is 66]

$$
\sigma_{T}^{*}(T)=\left\{\begin{aligned}
\sigma_{\min (\Delta t)} \cdot \sqrt{T}+D & \text { for } T \leq \min (\Delta t) \cdot L \\
A T^{3}+B T^{2}+C T+D & \text { otherwise, }
\end{aligned}\right.
$$

where $\sigma_{\min (\Delta t)}^{2}$ is the variance of the minimal transmission delay $\Delta t$. The dotted line in Fig. 6.3 shows a fit of the simulated data to Eq. 6.1, which is close to the lower envelope of all chains.

The data in our experiment shows a decreasing Weber fraction, so all intervals can be assumed to be encoded by the fastest synfire chain available. We thus use the first column of Eq. 6.1 to fit the data, resulting in values of $\sigma_{\min (\Delta t)}=7.13 \mathrm{~ms}$ and $D=6.87 \mathrm{~ms}$. The fit gives a very good description of the data averaged over participants (97.5\% of variance explained).

\subsubsection{Memory and decision stage}

\subsubsection{Stochastic framework}

We now formalize the adaptation in the serial memory system as an information processing model. A neural implementation of this system is in progress. The central stochastic variable is the difference $X_{i}(I)$ between the contents of the first and the second unit, where $I$ is the time index of the intervals and $i$ is the position of the deviant interval within the sequence. We use a general number of $N$ intervals (set to seven to fit the present data). The intervals are presented during the first $N$ time steps, while the computation of the difference $X_{i}(I)$ starts with the arrival of the second interval $(I=2)$ and is finished after $I=N+1$ to complete a total of $N$ comparisons.

Each interval represented by the spike patterns of the synfire chains is denoted by $S_{i}$ and can be considered as a Gaussian random variable with the actual interval duration as the mean and the variance determined by the timing error $\sigma_{T}$ (cf. Sec. 6.3.1). Assuming the same $\sigma_{T}$ for both standard and deviant interval, the VTI is given by

$$
S_{i}=S_{v}=\mathcal{N}\left(\bar{S}_{v}, \sigma_{T}^{2}\right)
$$

and the STI is

$$
S_{j}=S_{s}=\mathcal{N}\left(\bar{S}_{s}, \sigma_{T}^{2}\right), \quad j \neq i .
$$

With these definitions, we can write $X_{i}(I)$ in the general form

$$
X_{i}(I)=S_{I}-\sum_{j=1}^{I} \frac{S_{j}}{I} .
$$

The first term is the content of MU1 (the interval presented at position $I$ ), and the second term is the average in MU2 over all intervals presented before position $I$. 
The difference $X_{i}(I)$ between the two units can now be used to evaluate the current interval in MU1 based on the information accumulated in MU2: If the difference exceeds a decision criterion $K$, the interval is judged to be irregular, otherwise it is judged to be regular. The probability for an "irregular" judgment is thus given by

$$
P\left(X_{i}(I)>K\right)=\Phi\left(\frac{\bar{X}_{i}(I)-K}{\sqrt{\operatorname{Var}\left(X_{i}(I)\right)}}\right),
$$

where $\Phi$ is the standard normal distribution function and $\bar{X}_{i}(I)$ and $\operatorname{Var}\left(X_{i}(I)\right)$ are the mean and the variance of $X_{i}(I)$, respectively.

In the framework of signal detection theory, the probability of a "irregular" response given the VTI in MU1 would correspond to the hit rate, while the probability of the same response, given an STI in MU1 would be the false positive rate. However, we are more interested in the joint probability to judge the entire sequence of $N$ intervals as "irregular", since this response determines the $75 \%$ detection thresholds $\bar{S}_{v}$ in the experiment. This probability is given by

$$
P(\text { "irreg" })=1-P\left(\bigcap_{I=2}^{N+1}\left(X_{i}(I)<K\right)\right)=1-\prod_{I=2}^{N+1} P\left(X_{i}(I)<K\right) .
$$

The second equality holds under the assumption that all events are statistically independent. Note that Eq. 6.6 gives an implicit equation for the $75 \%$ detection thresholds $\bar{S}_{v}$ at each of the positions of the VTI, given the probability $P$ ("irreg"), and the set of parameters $\left\{\sigma_{T}, K\right\}$. $P$ ("irreg") is set to 0.75 in the current experiment, and the parameter set $\left\{\sigma_{T}, K\right\}$ can be used to fit the model to the experimental data.

\subsubsection{Results}

To use Eq. 6.6 for determining the $\bar{S}_{v}$ values, we must calculate the probabilities $P\left(X_{i}(I)>\right.$ $K$ ) for each value of $i$ and $I$, and thus, the means and variances of the respective variables $X_{i}(I)$. However, we can divide all possible combinations of $i$ and $I$ in three groups, each of which have the same mean and variance for all its respective members:

1. The VTI has not yet been presented at position $I(i>I)$. In this case, both MU1 and MU2 contain only STIs. Thus,

$$
X_{i}^{(1)}(I)=S_{s}-\sum_{j=1}^{I} \frac{S_{s}}{I} ; \quad \bar{X}_{i}^{(1)}(I)=0 .
$$

2. The VTI is presented at time $I(i=I)$. Now MU1 contains the VTI, while MU2 is the same as in 1):

$$
X_{i}^{(2)}(I)=S_{v}-\sum_{j=1}^{I} \frac{S_{s}}{I} ; \quad \bar{X}_{i}^{(2)}(I)=\bar{S}_{v}-\bar{S}_{s}
$$



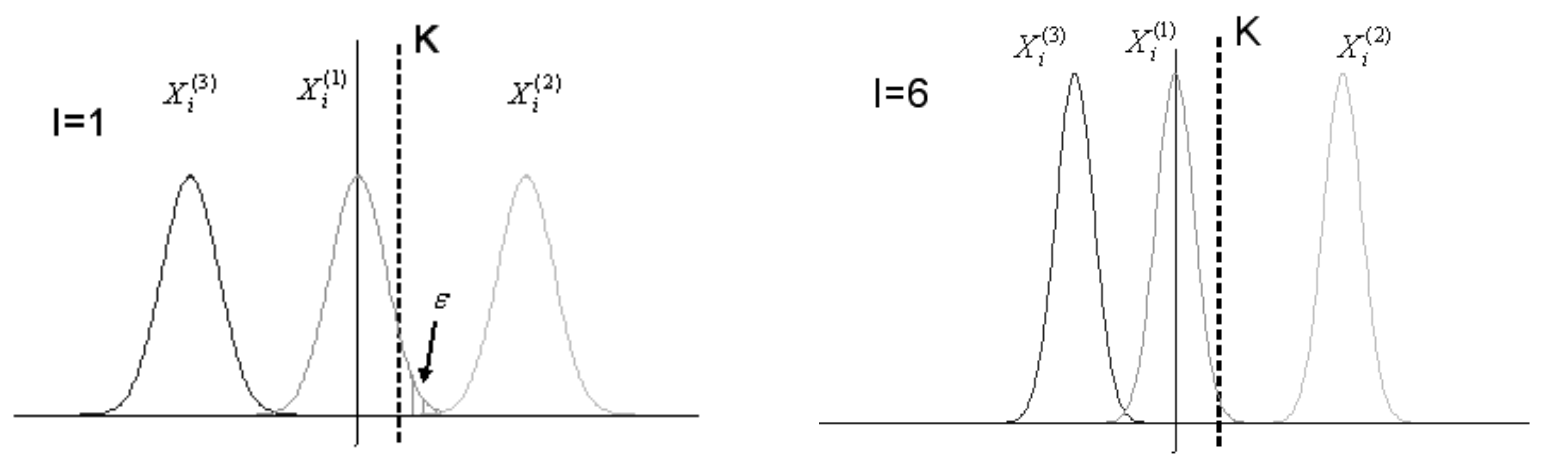

Figure 6.4.: Distributions of $X_{i}(I)$ for the three cases (see text) and two values of $I$.

3. The VTI has already been presented at an earlier position than $I(i<I)$. MU1 contains an STI, again, but one of the intervals in MU2 is the VTI. Thus,

$$
X_{i}^{(3)}(I)=S_{s}-\sum_{j=1}^{I} \frac{S_{s}}{I}-\frac{S_{v}}{I} ; \quad \bar{X}_{i}^{(2)}(I)=\frac{\bar{S}_{s}-\bar{S}_{v}}{I}
$$

The variance of the $X_{i}(I)$ does is the same in all three cases, because the variance $\sigma_{T}$ does not differ for the STIs and the VTI:

$$
\operatorname{Var}\left(X_{i}^{(1)}(I)\right)=\operatorname{Var}\left(X_{i}^{(2)}(I)\right)=\operatorname{Var}\left(X_{i}^{(3)}(I)\right)=\sigma_{T} \frac{I^{2}+I}{I^{2}} .
$$

Additionally, it must be noted that the criterion $K$ can not be entirely freely chosen. Specifically, it must be ensured that the probability of an "irregular" judgment is below the defined $P\left(\right.$ "irreg") if the sequence does not contain a deviant interval, or $\bar{S}_{s}-\bar{S}_{v}=0$. Otherwise, the adaptive method would make the detection thresholds converge to zero, as a sequence of regular intervals would be sufficient to elicit "irregular" responses with the defined probability. Together with Eq. 6.5 and 6.7, this requirement results in the following condition on $K$ :

$$
K>\sigma_{T}(1-P(\text { "irreg" }))^{1 / N} \approx 0.915 \sigma_{T},
$$

where the second equality holds for $P($ "irreg" $)=0.75$ and $N=7$.

\subsubsection{Approximation}

Plugging the results Eqs. 6.7, 6.8, 6.9 and 6.10 into Eq. 6.6 yields an equation that only depends on $\sigma_{T}, K$ and the detection thresholds $\bar{S}_{v}-\bar{S}_{s}$. This equation can be used to fit $\sigma_{T}$ and $K$ to the experimentally obtained thresholds. However, the relative contributions of the two parameters to the data will not be apparent in these equations. Here, we derive an approximation where these contributions can be more clearly seen. 
Table 6.1.: Fit parameters for Eqn. 6.6 (and others, see text).

\begin{tabular}{cccc}
\hline \hline STI duration $[\mathrm{ms}]$ & $\sigma_{T}[\mathrm{~ms}]$ & $K / \sigma_{T}$ & variance explained [\%] \\
\hline 50 & 45 & 0.923 & 87 \\
150 & 90 & 0.965 & 97 \\
250 & 110 & 1.0 & 89 \\
\hline \hline
\end{tabular}

Fig. 6.4 illustrates the three distributions of differences $X_{i}(I)$ for two values of $I$. All distributions become more peaked for later $I$ s as a result of the averaging process. Furthermore, the mean value of $X_{i}^{(2)}$ always reflects the actual difference between the VTI and the STI, while the mean of $X_{i}^{(3)}$ is the negative of this difference for $I=1$ and decreases in its absolute value for later $I$. Therefore, it is apparent that the false positive rate $\epsilon=P\left(X_{i}(I)<K \mid S_{i}=S_{s}\right)$ (shaded area in Fig. 6.4) is maximal for $X_{i}^{(1)}$.

Now assume that we have chosen the criterion $K$ such that $\epsilon$ never exceeds a certain value $\epsilon^{*}$ for $X_{i}^{(1)}$. Then, from the above observations, we see that $\epsilon^{*}$ is also the upper bound for the false positive rate for $X_{i}^{(3)}$, so we can consider $\epsilon \leq \epsilon^{*}$ for all $N-1$ false positive cases and approximate Eq. 6.6 by

$$
P(\text { "irreg" }) \geq 1-\left(1-\epsilon^{*}\right)^{N-1}\left(1-\Phi\left(\frac{\bar{S}_{v}-\bar{S}_{s}-K}{\sigma_{T}(I)}\right)\right),
$$

where $\sigma_{T}^{2}(I)$ is the position-dependent variance common to all three cases, as given in Eq. 6.10. Thus, the detection threshold is given for each $i$ by

$$
\bar{S}_{v}-\bar{S}_{s} \geq \Phi^{-1}\left(1-\frac{1-P(\text { "irreg" })}{\left(1-\epsilon^{*}\right)^{N-1}}\right) \sigma_{T} \sqrt{\frac{I^{2}+I}{I^{2}}}+K .
$$

From this equation, one can see that the threshold decreases with $I$ like $\sqrt{1+1 / T}$, while the steepness of the decrease is governed by $\sigma_{T}$ and a factor depending on $\epsilon^{*}, P($ "irreg") and the number of intervals $N$. Additionally, there is an offset that is equal to the criterion $K$.

\subsubsection{Fit to data}

We use Eq. 6.6 together with the results on mean and variance Eqs. 6.7, 6.8, 6.9 and 6.10 and the constraint on $K$, Eq. 6.11 to fit the parameters $\sigma_{T}$ and $K$ to the data set of the three different standard durations. The fits are depicted as solid lines in Fig. 6.1, The model gives a good description of the data averaged over participants. (see Table 6.1).

\subsection{Discussion}

We presented a model that can explain context effects on interval discrimination performance that we observed in a sequence experiment, and also the decrease of performance with increasing standard interval durations. Apart from the individual effects, the model also explains the 
interaction of the two: $\sigma_{T}$ increases with the STI durations and enters as a factor in Eq. 6.13, Thus, longer STI durations increase the steepness of the adaptation curve, and thus enhances the position effect.

Fitting Eq. 6.1 to the data suggested a very high temporal spread, $\sigma_{\min (\Delta t)}=7.13 \mathrm{~ms}$. This is about one order of magnitude higher than the values that we found to be realistic 66. However, this may be a specificity of sequence experiments, as the Weber fractions (0.49 to 1.18) are also very high compared to interval discrimination, where fractions between 0.05 and 0.2 are typical. A possible explanation lies in the rapid presentation of the stimuli: The ISI of maximally $250 \mathrm{~ms}$ might not be long enough to allow the intervals to be completely processed, causing an additional error.

The detection thresholds decrease with the position $I$ of the variable interval like $\sqrt{1+1 / I}$. Therefore, even for very long sequences, the variability will not be eliminated, but only decreased to a value close to $\sigma_{T}$ (cf. Eq. 6.13), the variability of a single interval. Therefore, the model could be falsified by data showing a drastically different form of decrease, e.g. linear or superlinear. Moreover, the model predicts that i) the saturation of the detection threshold should be apparent in longer sequences, and ii) that there should only be a limited effect in single-interval task such as interval production. We already confirmed the first prediction in an experiment with nine intervals [17].

On the other hand, the model is not directly falsified by the fact that it does not explain the end effect. Like other more complex effects [17], this could be included by introducing a decay of the representations in the MUs. At the final time step, no new interval is represented in MU1, so the comparison has to rely on the partly decayed memory trace of the second-to-last interval. Because of the decay, the variability of this representation will be increased, which explains the poor discrimination performance at the final position. 


\section{Discussion}

In this thesis, we attempted to extend the knowledge about the processing of temporal information in the brain into three directions. First, we investigated how neurocomputational models could be constrained by psychophysical findings regarding the relation between interval length and timing errors. By means of introducing a general stochastic framework (Chapter 4), we identified the optimal scaling behaviors of timing errors, given that the underlying stochastic processes in the brain are governed by their first and second moments, respectively. Furthermore, we proposed a neurocomputational model based on multiple synfire chains (Chapter 3) which is capable of reproducing the experimental finding of a U-shaped Weber fraction in the range of hundreds of milliseconds, by means of constrained optimization. Optimal selection of the chains could be accomplished by projections onto readout network which is subject to a specific plasticity rule. Second, we studied the possible connections between different modes of temporal processing, namely time perception in different sensory modalities and continuous motor timing (Chapter 5). Unlike previous studies which provided only correlational evidence, we used distortions of subjective duration by non-temporal factors to see if manipulations of temporal processing in one mode affected the other. We found an interaction between time perception in the visual and auditory domain, but no such links between time perception and continuous motor timing. Furthermore, we showed that a secondary motor task could impair discrimination of intervals in the range of hundreds of milliseconds. And finally, we studied the case of isochronous sequences of intervals and proposed a simple model that could account for our experimental observation that a deviant interval could be more easily detected when preceded by a larger number of standard intervals (Chapter 6).

\subsection{A general framework for temporal processing}

In Chapter 1, we briefly described the outlines of a general framework which could account for these and several other findings in temporal processing. Here, we discuss this framework in more detail, show how each of our results fit in, and which predictions could be derived from it.

The framework (Fig. 7.1) resembles the classical pacemaker-accumulator model (PAM, cf. Section 2.1.3.2) in the sense that it comprises separate processing stages for the actual representation of interval durations, memory storage, and comparison. However, the individual stages are more complex in our model, and partly also grounded in neural dynamics. The key conceptual differences to the PAM are the following:

1. There are multiple timing processes which are specific to sensory modalities, and also to a specific range of interval durations.

2. The temporal information from the individual processes are integrated in a centralized 


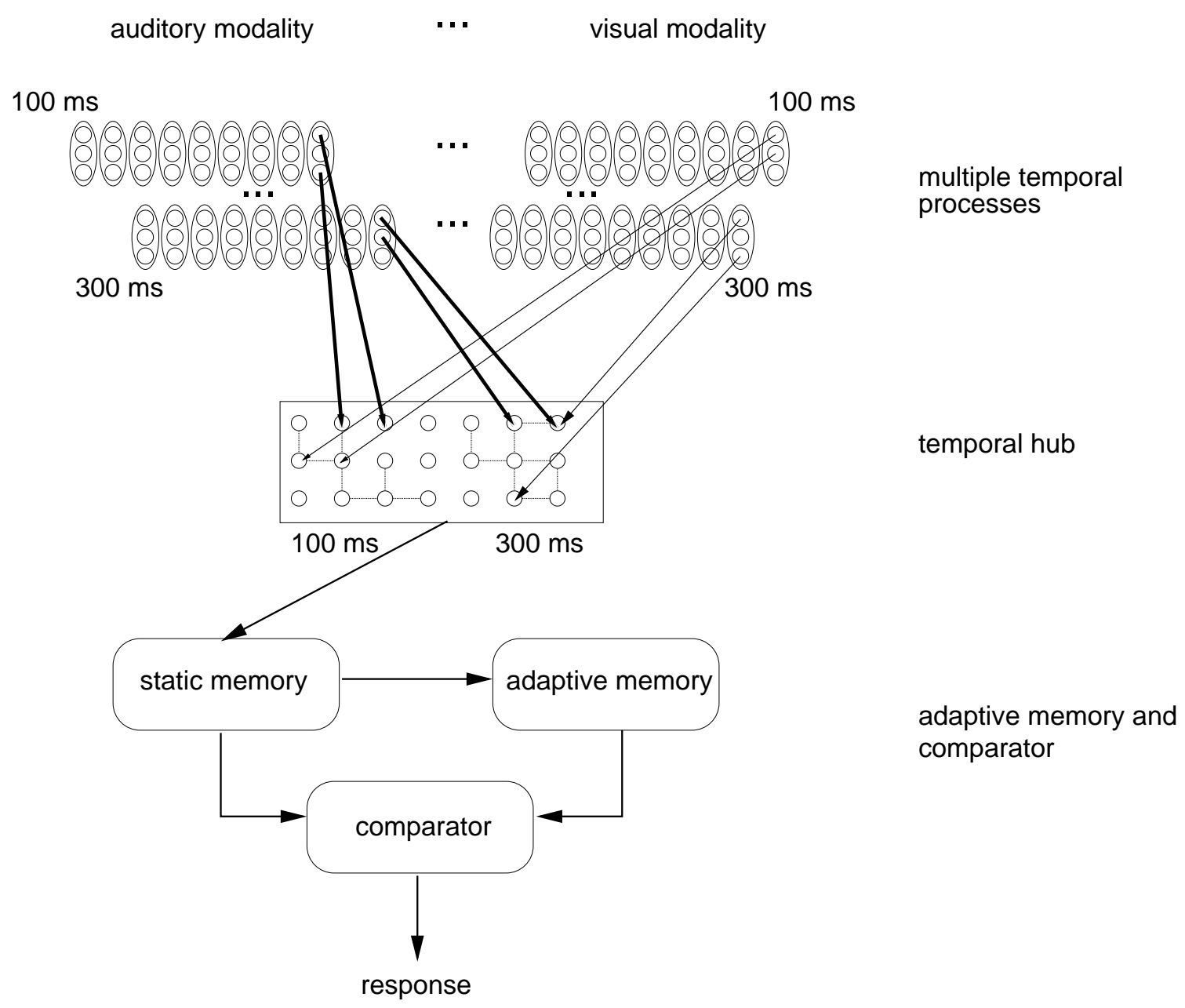

Figure 7.1.: Illustration of the general framework of temporal processing which emerges from our results. In the top part, multiple timing processes from different modalities are shown. As in Chapter [3, they are modeled as synfire chains. The time values correspond to chains that are optimized for that specific interval duration (cf. Section 3.4). The middle part depicts the temporal hub, a recurrent network where the timing processes project to (cf. Chapter 5). The synaptic weights (illustrated by the strength of the arrows) are determined by the reliability of each source. Here we show the case that auditory processes carry more temporal information than visual ones (cf. Section 2.1.2.3). Dotted lines denote recurrent connections within the hub which form cell assemblies for different intervals (100 and $300 \mathrm{~ms}$, here). Finally, the lower part of the figure shows the memory and comparator stage, which is identical to the classical PAM, with the exception that the reference memory integrates over intervals, forming a basis for averaging effects (cf. Chapter [6). 
"temporal hub" in a Bayesian way, i.e. they are weighted according to the respective reliability of each source.

3. The second memory unit (called "reference memory" in the PAM) is adaptive and integrates temporal information over a longer period of time.

In the following, we discuss each of these three components of the model in the light of our own results.

Multiple timing processes. In Chapter 3, we showed how the multiple timing processes could be neurally realized as synfire chains: The quasi-spatial position of an activity volley encodes the time since the activation of the first pool. As the timing errors increase super-linearly with the transmission delay within the chain, timing is optimal if only the last pool is used for each chain. However, the model is more flexible compared to other proposals of multiple timing processes, because the chains are not restricted to a single interval (as e.g. in the model proposed by Ivry [77]), but may also represent shorter intervals by using a number of pools near the end of the chain.

Despite of the favorable timing properties of synfire chains, we do not claim that they are the only possible mechanism that underlies temporal processing. This is most obvious when looking at the limitation of the range of intervals that can be represented with this model: Due to dynamical limitations of stability, this limit is around $700 \mathrm{~ms}$, although it may be probably be extended to more than a second with different parameter combinations. At longer intervals, other mechanisms of time perception have to take over, e.g. ramping activity (cf. Section 2.2.3). This may be one source of the recurrently observed differences between millisecond and second timing (cf. Section 2.1 and 2.2). But even in within the millisecond range, we assume that all the various sources of temporal information, such as oscillations, short-term synaptic plasticity, or slow ion channels may be exploited (see Section 2.2.3 and Chapter 3). As the only constraining factor, we demand that the sum of the temporal information obtained in this way complies with the scaling behavior of the psychophysical timing error. Under the assumption of an ideal observation, the stochastic framework we presented (Chapter 4) provides a useful tool for testing this constraint: The information from each of the processes used can be quantified by the Fisher information, and the sum of these information measures constitute the net Cramer-Rao bound, which would be reached in an ideal observation. Thus, the proposed combination of sources provides a valid model for temporal processing when the Cramer-Rao bound scales in the same way as the psychophysical timing error. This analysis is further simplified by the fact that the optimal scaling behavior is governed by the best process involved for each interval of time (cf. Section 3.4 and Section 4.4): When adding another timing mechanism to a number of existing ones which produce the correct scaling behavior, all one needs to show is that the new process does show the same, or a worse (i.e. steeper) scaling. Note, however, that even if the new process does not affect the scaling behavior of the error, it may still contribute valuable information that reduces the absolute value of the error, i.e. the prefactors of the scaling law.

The key element for any given model to reproduce a U-shaped Weber curve is a superlinear component of the timing error which becomes relevant at longer intervals. In the synfire model, the constraint was given by a finite chain length and a steeply increasing timing error 
with the transmission delay. So far, no other neurocomputational model has been shown to have such a superlinear error component. Weber's law, on the other hand, can be reproduced more simply by using a stochastic process which evolution is governed by its variance only (cf. Chapter 4). Using this form of temporal information may be necessary when the mean of the process is not changing with time, or if the noise is so strong that its information is obscured. Moreover, recent investigations show that stimulus properties encoded in the variance of neural activity may lead to a more rapid transmission, as needed for certain fast ecological decisions [160]. In this case, accuracy of the time estimate would be traded off for transmission speed. Further electrophysiological investigations could clarify the exact role of variance-encoding for temporal processing.

Temporal hub. The temporal hub is the key element in our framework, as it constitutes a centralized site where all temporal cues about a given (potentially multi-modal) stimulus converge and form an integrated estimate of the duration of an interval. The hub takes the form of a recurrent neural network with input projections from all the respective sources of temporal information. This information is optimally integrated when the respective means are weighted with the reliability, or inverse variance of each source (cf. Section 2.3.3). This weighting could be realized at least in two ways. One possibility is to use variance-encoding inhibitory neurons ([160], see above), which perform the division in Eq. 2.24] directly by means of shunting inhibition [72]. As the reliability of each source is computed online, this constitutes a very flexible way of implementing Bayesian integration, which is necessary in cases where the variability of sources changes rapidly [45]. On the other hand, it may also be beneficial to store long-term tendencies in the reliability of the sources. For instance, as we have shown in Chapter 3, the input from a faster synfire chain at a given interval duration is always more reliable than the input from a slower one. For such cases, the weighting can be directly translated into synaptic weights of the projections into the hub. In Section 3.5, we have shown how such are variance-dependent scaling of synaptic weights could be achieved by a combination of STDP and homeostatic plasticity. It may be rewarding to further explore the possible applications of this kind of long-term variance encoding outside the field of temporal processing.

Note that in Fig. 7.1, there are projections from the inputs at 100 and $300 \mathrm{~ms}$ from both modalities onto the same regions in the temporal hub, respectively, but no other connections (e.g. from both 100 and $300 \mathrm{~ms}$ inputs in the same modality onto the same region in the hub). This connectivity does not require $a d$ hoc pre-wiring, but may follow from competitive synaptic plasticity (cf. Section 3.5), if we assume that the recurrent connections within the hub are plastic as well: When the configuration starts with all-to-all connections, initially all regions in the hub will be activated alike by the inputs. However, due to fluctuations and synaptic competition within the hub, this symmetry will eventually break and cell assemblies [1] form which lead to a stronger response to an input within the assembly compared to cells outside of it. Because of the Hebbian synaptic competition of the projections into the hub, only those connections into a cell assembly will be strengthened. Other connections will be weakened by homeostatic plasticity, and may disconnect entirely by means of synaptic turnover. The exact dynamics of these plasticity rules should be investigated in further modeling studies.

The assumed plasticity within a hub also provides an explanation for the effect of cross- 
modal training: Several studies report that training e.g. of an interval discrimination task within one modality also improves discrimination in another modality, but these effects do not generalize to other interval durations (see [80, 28, for reviews). The second observation was already discussed in Section 3.6. Assuming competitive plasticity of connections also within the hub, the observation of cross-modal generalization can also be explained: Training within one modality does not only strengthen the input connection of the corresponding process into the hub, but also the recurrent connections of the cell assembly that encodes the respective interval. So when the same interval is presented in another modality after the training phase, it will profit from the strengthened connections within the hub. From this logic, we could draw the experimental prediction that the performance differences between pre- and post-training test would be stronger when tests are conducted in the trained modality compared to another modality. This is because in the training modality, both the connections of the input and within the hub are enhanced. To our knowledge, this comparison has not been made so far.

While the model of the temporal hub is a quite general one, it is still specific enough to be tested, and potentially, to be falsified: An alternative explanation for cross-modal interaction is given by direct synaptic interactions between modality-specific temporal representations. A critical experiment to decide between these two explanations would be to present intervals within two modalities at the same time and distort the subjective duration in one of the modalities. Within our experimental setup (Chapter 5), this distortion could be induced by observed motion in the visual modality. If the temporal information from the two sources is integrated into a temporal hub, subjective duration would be the same in both modalities for all levels of distortion. But if the two modalities directly interact with each other, the two duration estimates may migrate towards each other, but will stay different, at least for some level of distortion.

Adaptive memory system. The memory system in our model is the same as in the classical PAM, with the exception that the reference memory unit integrates over all previously presented intervals instead of storing a single interval. In Chapter 6, we have shown that this architecture provides an explanation for adaptation effects in detecting a deviant interval within an isochronous sequence. Further experiments by our collaborator Stefan Blaschke have also shown more complex effects [17, 16] which could be explained by extending the model. For instance, he presented participants with sequences comprising three different standard durations, and investigated the differences between the a presentation where the different durations were either blocked or randomized. For 50 and $250 \mathrm{~ms}$, he found that the randomized presentation yielded worse detection results, as one might expect. But interestingly, for 150 ms, which is the arithmetic mean between the other two standard durations, no such difference was observed. We concluded that the individual intervals are not directly compared with one another, but that participants develop a long-term standard that averages over the entire set of trials that were presented during the experiment. This effect can be incorporated into the model by assuming a longer time constant of the integration. The details of these modifications are currently being investigated.

So far, we did not make any statements about the anatomical locations of each of the components of the model. From previous neuroscience studies (cf. Section 2.2.1), it seems 
reasonable to assume that the individual timing processes are located in modality-specific regions of the neocortex. However, it may also well be possible that other structures such as the cerebellum contribute their potential to encode temporal information. In this view, the cerebellum would not have an exclusive role in temporal processing, but could still be dominant for tasks which are well suited for its specific architecture, such as the eyeblink reflex (cf. Section 2.2.1). In other tasks, the role of the cerebellum may be of more general nature, such as the correction of motor errors [89].

A series of reviews by Meck and coworkers [119, 24, 142] provide strong evidence that the basal ganglia is involved in temporal processing in a way that temporal information from various cortical areas converges to the striatum. While Meck assumes that the striatal spiny neurons perform coincidence detection of cortical oscillations (cf. Section 2.2.3), we propose that these neurons constitute an ideal neural basis for the temporal hub, which performs similar computations as in the Meck model, but with more general input. The proposal that the basal ganglia acts as a temporal hub could be tested in clinical and lesion studies: Lesions in the spiny neurons should lead to impaired performance when multi-modal stimuli or stimuli from very different ranges of durations are combined.

We do not have any strong evidence for the exact neural instantiation of the memory and decision components, other than the common proposal that such executive functions reside within the prefrontal cortex [89]. This view is supported by the observation that activity from cortico-striatal pathways are eventually fed back into the cortex [154].

\subsection{Conclusion}

In the beginning of this thesis, we formulated five questions that we considered central for the current research on temporal processing: What is the transfer function between physical and subjective time, what role do non-temporal factors plays, what are the neural codes and the brain structures used for temporal processing, and finally, are all these aspects common to all variants of temporal processing? We conclude this thesis with a discussion of how our work may have contributed to answer any of these questions.

In Chapter 3 and 4 , we showed that the question about the transfer function and the neural code are best answered together. We both proposed a neural mechanism from which the experimentally observed error function emerges, and identified constraints of the neural model that were necessary to account for these experimental findings. Furthermore, we showed how non-temporal factors such as attention and training could modify temporal estimates within the model. In Chapter 5, we used the non-temporal factors motion and attention to assess the question of whether temporal processing relies on centralized or distributed mechanisms. We found evidence for a third option: Bayesian integration of temporal information from distributed sources into a centralized temporal hub. And finally, in Chapter 6, we showed that the representation of sequences can be reduced to the representation of single intervals, so no separate mechanism is necessary for this task. Only the timing of continuous motion seems to rely on a such a separate mechanism, as we showed in Chapter 5. The only question we could not directly address is the one about the anatomical localization of temporal processing, but we still were able to deduce some likely locations for each of the components of our new architecture (see above). 
This works favors a view onto temporal processing and time perception which may be novel in some respects: The processing of temporal information is as ubiquitous in brain as processes that are changing in time in the outer world. The brain can not afford to waste any of these information, but has to integrate all of them as well as it can. Only such an integrated judgment about time allows to coordinate our senses and actions with a world that is constantly changing. In this sense, the sense of time emerges from multiple sources, unlike the sense of sound or the sense of touch, which is represented in more specific way. Conversely, time can not be seen as an "a priori notion" that enables all sensation, as Kant believed, but time is a percept itself that is shaped and manipulated by these very sensations. Taking this view may not reduce the number of questions that need to be answered about the nature of time perception, but hopefully allows to ask some them in a more specific way. 
Appendix 



\section{A. Supplemental material for Chapter 5}

\section{A.1. Materials and Methods}

Participants. 20 adult volunteers took part in Experiment 1 to 4 , and Experiment $1 \mathrm{~b}$ comprised 10 participants (90 participants in total, 76 women and 14 men, mean age 23.3 years, ranging from 19 to 40 years). All had normal or corrected-to-normal vision and normal hearing. They were naïve to the purpose of the experiment.

Apparatus and Stimuli. All experiments were controlled by a $\mathrm{C} / \mathrm{C}++$ program running on a computer operating on SuSE Linux 9.0 (SuSE Linux) and a haptic device (Phantom Premium 3.0L 6DOF, SensAble Technologies) connected by a parallel port. The internal clock of the haptic device, which updates the state of the robotic arm every millisecond (update frequency $1000 \mathrm{~Hz}$ ), controlled the timing of the experiments.

All experiments were performed standing $50 \mathrm{~cm}$ away from of a computer screen (FujitsuSiemens Computers, Scenicview P19-2), holding the end effector of the haptic device in the right hand, and wearing headphones (Technics RP-FT30). In experiments containing a motion task, arm motion was performed in the sagittal plane, and recorded in 3D by the manipulandum (Fig. A.1). In experiments containing a time perception task, participants listened to white-noise bursts generated with an external sound generator and presented binaurally through the headphones with an intensity of $65 \mathrm{~dB}(\mathrm{~A})$. To avoid interference of the arm movements with a motor response, the participants articulated their responses to the time perception task verbally. The experimenter recorded each response by pressing the corresponding button on a keyboard.

In all experiments, the participants were presented with an OpenGL 3D environment on the screen (Fig. A.2). They watched a blue sphere $(0.8 \mathrm{~cm}$ of width and height in screen coordinates) moving clockwise on an elliptic trajectory (called "target"). The length of the main axes of the ellipse on the screen were 20 and $10 \mathrm{~cm}$, respectively. In all experiments except Experiment 2, the angular velocity of the sphere was constant at $2 \mathrm{rad} / \mathrm{sec}$. This results in a tangential velocity that varies periodically, with a maximum of $20 \mathrm{~cm} / \mathrm{sec}$ at the upper and lower apices and a minimum of $10 \mathrm{~cm} / \mathrm{sec}$ at the left and right apices. In Experiment 2 , the tangential velocity was kept constant to $15 \mathrm{~cm} / \mathrm{sec}$. The total time for one revolution was $3.14 \mathrm{sec}$ in all experiments. There were also two static ellipses drawn in yellow which surround the trajectory (main axes $22 \mathrm{~cm}$ and $12 \mathrm{~cm}$ for the larger ellipse, $18 \mathrm{~cm}$ and $8 \mathrm{~cm}$ for the smaller ellipse), and finally, a red sphere ("proxy") of the same size of the target sphere, which could be controlled by moving the end effector of the robotic arm (Fig. A.2).

General procedure. All participants performed a series of three experiments: One with a time perception task only (time), another with a motor task only (motion), and a third one 


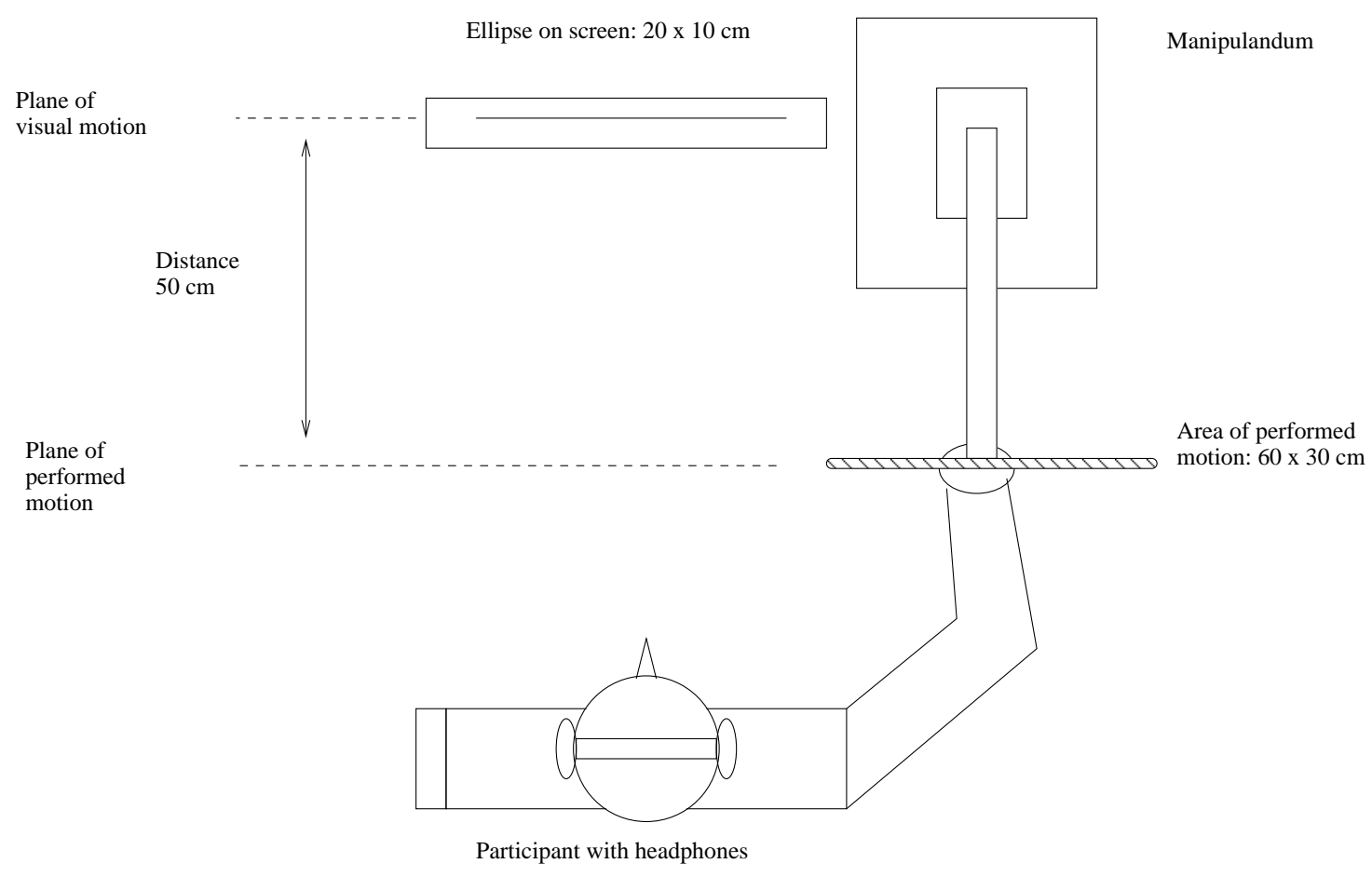

Figure A.1.: Setup of the experiment. Participants stand in front of a screen wearing headphones. They moved the end effector of a robotic manipulandum in order to move a proxy on the screen. Setup of the experiment. Participants stand in front of a screen wearing headphones. They moved the end effector of a robotic manipulandum in order to move a proxy on the screen.

with both tasks combined (time_motion or $t m$ ). The order in which these experiments were performed was balanced over participants. There was a brief training phase for the time and the motion task before the actual experiments started. Breaks of one minute duration were taken after every five minutes of performing each experiment, or earlier if the participants requested it.

Time experiment. A trial consisted of two white-noise stimuli, the first one with a standard interval of $100 \mathrm{~ms}$ (SI) between the onset and the offset of the stimulus, and the second one with a comparison interval (CI). The stimuli were always presented at one of the four apices of the ellipse trajectory of the target sphere. More specifically, the onsets of a stimulus of duration $T$ were timed such that the sphere passed one of the apices of the elliptic trajectory at $T / 2$ (see Fig. A.2). The four possible positions of the first stimulus (upper, lower, left and right apex) constitute four different conditions, and the second stimulus is always presented at the following apex (see Fig. A.2 for an example). In two of the conditions, the first stimulus is presented in one of the less curved apices (called straights) and the second stimulus in one of the more curved ones, and in the other two conditions, this order was reversed (called curves). These four conditions were assigned to the individual trials with equal probability, and no 


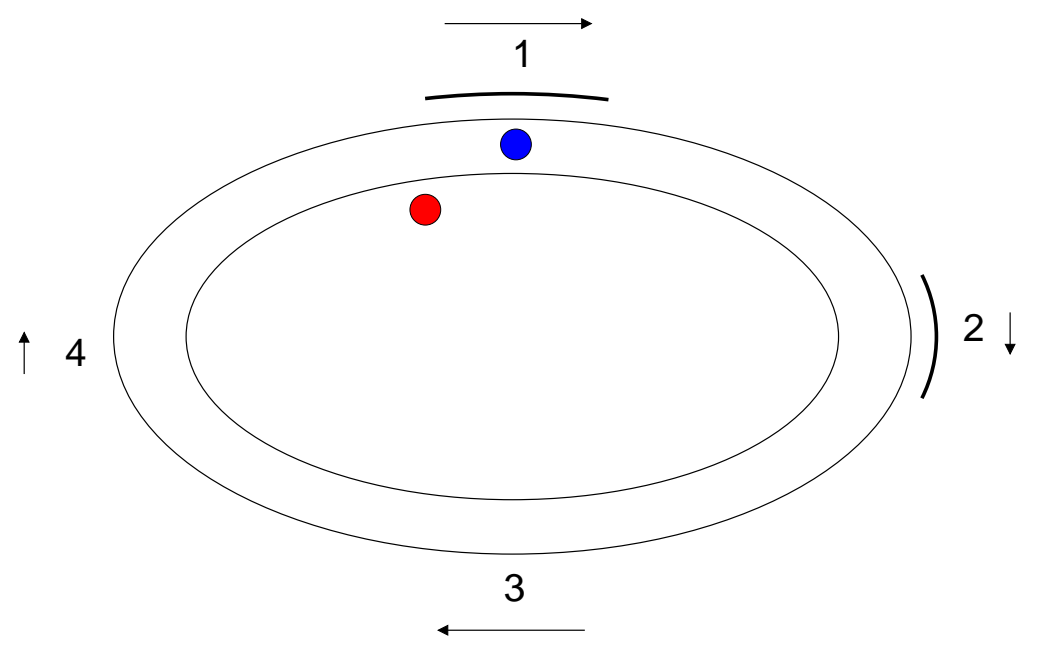

Figure A.2.: Screen contents for visual feedback during the motor task. The blue sphere (target) moves along an elliptic trajectory at a prescribed speed. The red sphere (proxy) can be moved with a robotic manipulandum. The two ellipses surround the trajectory of the blue sphere to mark an area that should not be left by the red sphere. Numbers depict the four possible positions of the first auditory stimulus for the interval discrimination task. The second stimulus followed at the subsequent apex. Position 1 and 3 are termed straights throughout this chapter, position 2 and 4 curves. As an example, the white patches depict the case that the first stimulus is presented at the upper straight. The arrows depict direction and magnitude of tangential velocity of the blue sphere in Experiment 1. The numbers, patches and the arrows were not actually shown on the screen, and the ellipses were shown in yellow against a black background.

distinction was made between the conditions for data analysis (see below).

The participants were instructed to decide which of the two stimuli was longer and say "first" if they perceived the first stimulus as longer and "second" if the second one appeared longer to them (two alternative forced choice). The duration of the CI was adjusted according to an adaptive rule [86] to estimate the mean CI time at which the participants answer correctly with a probability of 0.75 . There were two separate runs in which the CI was initialized at 130 or $70 \mathrm{~ms}$, respectively. In both runs, the distance of the CI to the SI was increased after a wrong judgment ("step-up"), and decreased after a correct one ("step-down"). More specifically, a judgment was considered as wrong whenever the participant answered "first" in the run starting at $130 \mathrm{~ms}$, or "second" in the run starting at $70 \mathrm{~ms}$. Based on the results of a pilot study we chose a step-up size of $15 \mathrm{~ms}$ and a step-down size of $5 \mathrm{~ms}$. There was a number of 32 trials for both runs, and trials from both runs were presented in randomized order, resulting in a total number of 64 trials. No feedback was given. The participants were instructed not to follow the target sphere with the proxy, but to keep the proxy in the centre of the ellipses. 
Motion experiment. Different from the time experiment, there were no acoustical stimuli and the participants were now instructed to follow the target sphere with the proxy by moving the end effector of the haptic device in the sagittal plane (Fig. A.1). They were instructed to follow the trajectory of the target as closely as possible, but also to keep the proxy between the two yellow ellipses which surround this trajectory at all times, and to move smoothly. After a few revolutions, when the participants felt comfortable with the motion, a countdown of $5 \mathrm{~min}$ was started during which the trajectory of the proxy sphere, its velocity and the distance from the target sphere was recorded. There were no constraints on the motion, but measurements of all three coordinates of the movement confirmed that participants largely confined their movements to two dimensions. The dimensions of the ellipse in motion space were approximately $60 \mathrm{~cm}$ in width and $30 \mathrm{~cm}$ in height (Fig. A.1).

Time_motion experiment. In the time_motion experiment, participants were required to perform both of the above tasks simultaneously, i.e. to follow the target sphere with the proxy and to respond to the acoustic stimuli. Both parts of the experiment were set up similarly to the conditions in time and motion, with some exceptions: First, the onset of the acoustic stimuli was now determined by the position of the proxy sphere, and not by the position of the target sphere as it was the case in the time experiment. The onset angle was calculated in the same way as in time, and the stimulus was started as soon as the proxy sphere passed this angle, and stopped again after the stimulus duration T. Second, the time task was run independently for each of the four conditions of motion (see time experiment), and each of these conditions was analyzed individually (see below). Each condition comprised 64 trials, which results in a total number of 256 trials. Finally, a response to a trial in the time task was recorded only if the distance of the proxy from the target did not exceed a certain value, which we set to $7.5 \mathrm{~cm}$ of screen coordinates based on the results of a pilot study. If this distance was exceeded during the presentation of one of the two stimuli, the current trial of the time task was repeated. The participants were instructed to do both tasks with the same priority, and that a bad performance in any of the two would prolong the duration of the total experiment.

\section{Procedures for the different experiments.}

Experiment 1. This experiment was performed according to the general procedure in all respects.

Experiment 1b. The elliptic trajectory of the target sphere and the yellow ellipses were rotated by 90 degrees such that the absolute positions of straights and curves were reversed.

Experiment 2. The angular velocity of the target sphere was manipulated such that its tangential velocity was kept constant to $15 \mathrm{~cm} / \mathrm{sec}$.

Experiment 3. The time experiment was changed such that the time perception task was performed and analyzed independently for the four different conditions (i.e. the four apices at which the first stimulus could appear). There were 64 trials for each condition, resulting in a total number of 256 trials. In this respect, the time experiment was matched to the time_motion experiment.

Experiment 4. The training phase for the motion task was prolonged such that all participants reached a common level of performance. We measured the target deviation $\Delta r$ (see 
below) at each millisecond and updated a measure of the accumulated target deviation $\Delta r_{c}$ in each time step $t$ according to the following formula

$$
\Delta r_{c}^{t+1}=\alpha \Delta r+(1-1 / \tau) \Delta r_{c}^{t}
$$

$\Delta_{t}$ started at an initial value of $\Delta_{t}^{0}=333 \mathrm{~cm}$ (screen coordinates) and the training phase was run until $\Delta r_{c}$ falls below half this initial value. Being updated by the above rule, $\Delta r_{c}$ increases in each time step with the currently measured target deviation, weighted by an increase rate $\alpha$ (set to $2.5 \mathrm{E}-5 \mathrm{~cm}$ ), and decays exponentially with a time constant $\tau$ (set to 2 $\min$ ). At these values $\Delta r_{c}$ decays to its half value (and stops the training phase) in 2 min if the participant keeps the target deviation at zero at all times. Non-zero target deviations increase $\Delta r_{c}$ according to the weighting factor $\alpha$. The value of results in a training phase that is not much longer than $2 \mathrm{~min}$ if the distance from the proxy to the target is kept below $7.5 \mathrm{~cm}$ at all times. That value was also used as a performance threshold in the time_motion experiment (see above). Applying this procedure resulted in training phases with a mean length of 4.4 min (SD $2.3 \mathrm{~min}$ ), as compared to $2.6 \mathrm{~min}$ (SD $0.9 \mathrm{~min}$ ) in Experiment 1.

Data analysis. We used Microsoft Excel 2003 (Microsoft Cooperation) for collection and basic analysis of the data, MATLAB R2008b (The MathWorks) for calculation of the motion parameters and R 2.8.0 (The R Foundation for Statistical Computing) for statistical analysis of all the data. Effect sizes of statistical effects were computed by Cohen's $d$ [31] for t tests and by partial $\eta^{2}$ [94 for analysis of variance, abbreviated as $\eta_{p}^{2}$ in the text.

Time task. The adaptive procedure used in the time task was designed to let the duration of the CI converge to the value where the participant judges the comparison interval as the longer one in $75 \%$ and $25 \%$ of all cases for the CI starting above and below the SI, respectively. We calculated the mean CI for both of these runs in each condition (time and the four positions in time_motion) based on the 20 last trials to exclude transient effects. From these values $\mathrm{CI}_{75}$ and $\mathrm{CI}_{25}$, we calculated the difference limen (DL)

$$
\mathrm{DL}=\frac{\mathrm{CI}_{75}-\mathrm{CI}_{25}}{2}
$$

and the point of subjective equality (PSE)

$$
\mathrm{PSE}=\frac{\mathrm{CI}_{75}+\mathrm{CI}_{25}}{2} .
$$

Motion task. We analyzed the trajectories of the motion experiments in two ways. First, we averaged over all points in the trajectory that correspond to a given angular position of the target. The target sphere took the finite number of 3142 such angular positions. For each of these angles, we calculated the mean and standard deviation of the deviation of the actual trajectory from the target trajectory $\Delta r$ ("target deviation"), measured by the Euclidean distance

$$
\Delta r=\sqrt{(x-\hat{x})^{2}+(y-\hat{y})^{2}}
$$

where $x$ and $y$ are the Cartesian coordinates of the proxy sphere and $\hat{x}$ and $\hat{y}$ are those of the target sphere. 
The second way to analyze the trajectories was to cut them into pieces that correspond to individual revolutions, or laps. For constructing a lap, the angular position of the target sphere is not suitable, as the movement of the proxy is usually faster or slower than the target sphere, so no given set of 3142 subsequent data points could be expected to yield an exact lap. Instead, we determined the laps based to the variable radius of the ellipse. The first lap started at the first point where the radius was minimal with the $y$ position greater than zero (upper apex), and ended at the next point where this condition was met again. For each data point of one of these laps, we calculated the curvature

$$
\kappa={\frac{\left|x^{\prime} y^{\prime \prime}-y^{\prime} x^{\prime \prime}\right|^{3 / 2}}{x^{2}+y^{\prime 2}}}^{2}
$$

and the tangential velocity

$$
\nu=\sqrt{\dot{x}^{2}+\dot{y}^{2}}
$$

where $\dot{x}$ and $\dot{y}$ are the approximated time derivatives of the $x$ and $y$ coordinate, respectively, and $x^{\prime}$ and $y^{\prime}$ are the approximated derivatives with respect to the angle $\theta$ around the origin of the ellipse, relative to the upper straight apex. In this way, the values of $\kappa$ and $\nu$ are given as functions of the radius $r$ of the trajectory of the proxy rather than the angular position of the target sphere. For each value of $r$, we average curvature and tangential velocity over all laps. Specifically, the values at the apices are determined by the four extreme values of the radius.

\section{A.2. Supporting text}

Data on individual conditions. In Table A.1 to A.4, we present the mean and variance of the PSE and DL computed for each individual condition (Table A.1 for PSE and Table A.3 for DL), and the pooled values of PSE and DL over the two straights and curves conditions, respectively, and over all conditions within the time and $t m$ experiment, respectively (Table A.2 for PSE and Table A.4 for DL). We also included Experiment 1b (see below), and presented the time and $t m$ condition in Experiment 3 separately. Note that in some experiments, namely Experiment 1 and Experiment 3 (time condition), the PSE is somewhat larger in the upper straight compared to the lower straight, violating the implicit assumption in our analysis of both straights and curves being identical. To resolve this possible issue, we performed Experiment $1 \mathrm{~b}$.

Experiment 1b. The PSE difference between upper and lower straight in some experiment raises the concern that the observed PSE differences between straights and curves may be related to the absolute position of the target on the screen, rather than differences in visual motion speed as we concluded. To exclude this possibility, we performed a control experiment with 10 additional participants where we rotated the elliptic trajectory by 90 degrees such that the position of the target shifted for all conditions: The two straights were now to the left and the right of the screen, and the curves were positioned at the upper and lower part. If the position of either the visual target or of the arm movement during the auditory stimulus presentations affected subjective duration, the PSE would change in opposite way in this 
setup compared to the original experiment, or at least show a less pronounced distortion. In contrast to this hypothesis, the PSE decreased from straights to curves, just as in Experiment $1(t(19)=-3.1, P<0.006$, Cohen's $d=0.46)$. From this result, we conclude that absolute spatial position has no notable effect on the PSE compared to visual motion speed.

Correlations between time and motion data. To directly correlate time and motion data, we extracted those pieces from the trajectories which were passed during the presentation of the two auditory stimuli of each trial in Experiment 1. We calculated the average of curvature and tangential velocity for the trajectory piece corresponding to the first and the second stimulus, respectively. Then, we correlated the difference in these measures between to first and second tone with the PSE and the DL. The PSE was significantly correlated with the differences of both parameters of motion between the first and the second stimulus (curvature: $r=-0.33$, $t(68)=3.1, P<0.003$, tangential velocity: $r=0.32, t(68)=3.0 P<0.004)$, but there was no significant correlation of the DL with neither the curvature $(r=0.16, t(68)=1.4, P=0.16)$ nor the velocity differences $(r=-0.16, t(68)=1.5, P=0.15)$. 

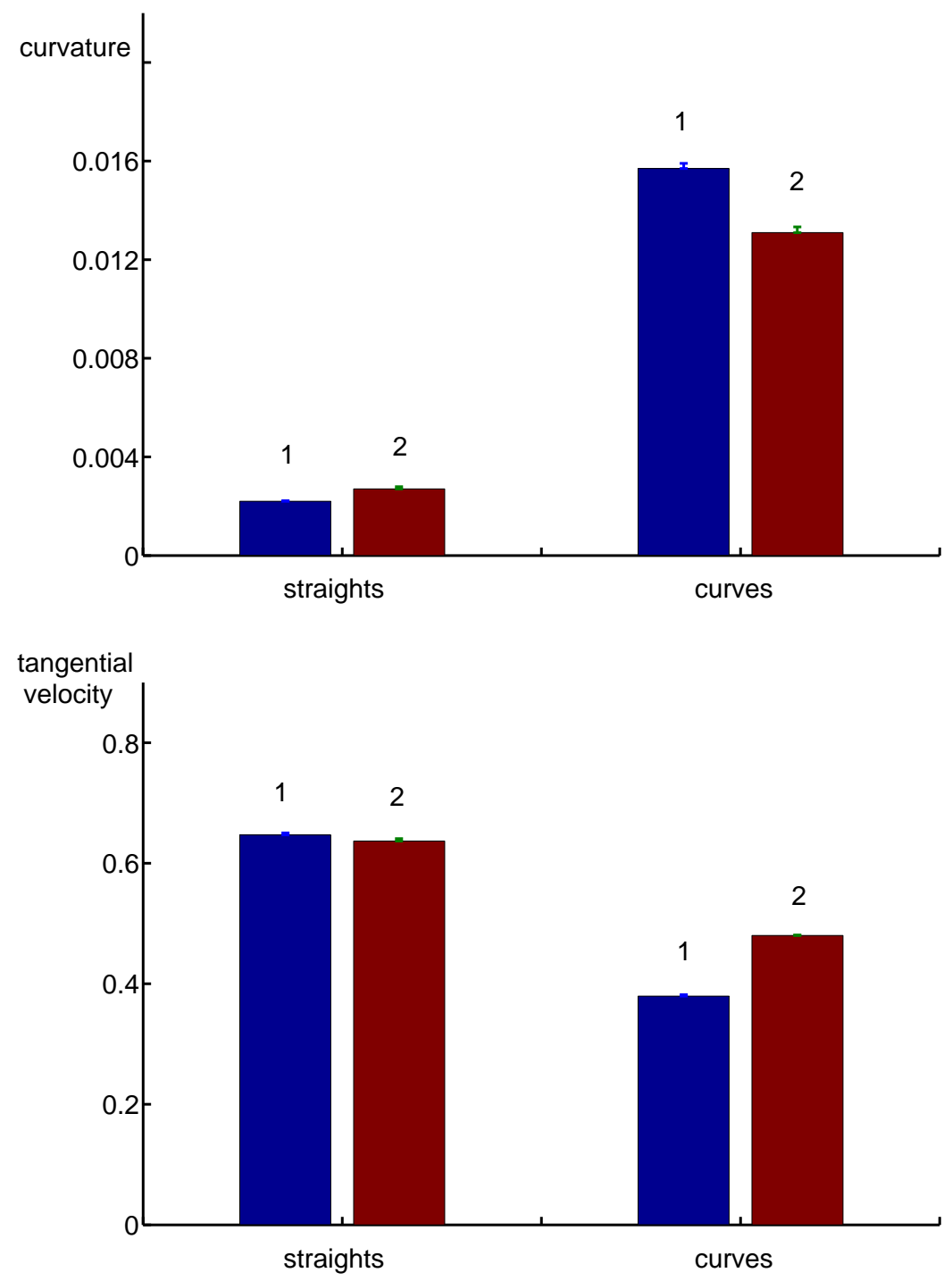

Figure A.3.: Curvature and tangential velocity for the straights and curves conditions, respectively, in Experiment 1 and 2. Error bars are standard errors. All motion parameters changed significantly from straights to curves $(P<0.05)$. These changes were less pronounced in Experiment 3 compared to Experiment 2. In Experiment 3 and 4 , the motion parameters showed the same qualitative behavior as in Experiment 1 (data not shown). 


\begin{tabular}{l||llll} 
Exp & $\begin{array}{l}\text { upper } \\
\text { straight }\end{array}$ & $\begin{array}{l}\text { right } \\
\text { curve }\end{array}$ & $\begin{array}{l}\text { lower } \\
\text { straight }\end{array}$ & $\begin{array}{l}\text { left } \\
\text { curve }\end{array}$ \\
\hline \hline 1 & $108.7(8.2)$ & $105.5(9.7)$ & $104.8(8.3)$ & $102.6(10.1)$ \\
$1 \mathrm{~b}$ & $108.6(12.1)$ & $101.1(12.6)$ & $107.7(11.6)$ & $105.1(9.9)$ \\
\hline 2 & $110.1(12.9)$ & $109.7(11.3)$ & $109.7(12.0)$ & $110.8(11.1)$ \\
\hline 3, time & $102.9(6.1)$ & $102.1(5.5)$ & $104.5(5.0)$ & $102.4(6.2)$ \\
3, tm & $109.5(9.1)$ & $104.9(10.0)$ & $106.3(10.6)$ & $105.2(9.7)$ \\
\hline 4 & $105.7(11.6)$ & $103.2(12.9)$ & $104.1(11.2)$ & $100.1(11.7)$
\end{tabular}

Table A.1.: PSE (in ms) for each individual condition and experiment. Each cell contains the average over all participants, and standard deviation in brackets. In Experiment 3 , the time and $t m$ condition are reported separately.

\begin{tabular}{l||ll||ll}
\multicolumn{1}{l||}{ Exp } & tm & time & straights & curves \\
\hline \hline 1 & $105.4(8.3)$ & $103.5(8.3)$ & $106.8(7.9)$ & $104.0(9.4)$ \\
$1 \mathrm{~b}$ & $105.6(10.8)$ & $104.6(8.0)$ & $108.1(11.6)$ & $103.1(10.4)$ \\
\hline 2 & $110.1(11.3)$ & $106.6(10.6)$ & $109.9(11.8)$ & $110.2(11.0)$ \\
\hline 3, time & $106.4(9.3)$ & $103.0(5.3)$ & $103.7(5.3)$ & $102.2(5.5)$ \\
3, tm & $106.4(9.3)$ & $103.0(5.3)$ & $107.9(9.5)$ & $105.0(9.4)$ \\
\hline 4 & $103.3(11.0)$ & $101.3(10.8)$ & $104.9(10.8)$ & $101.7(11.9)$
\end{tabular}

Table A.2.: PSE (in ms) for each experiment averaged for time, tm, straights and curves condition. Each cell contains the average over all participants, and standard deviation in brackets. In Experiment 3, the time and $t m$ condition are reported separately. 


\begin{tabular}{l||llll} 
Exp & $\begin{array}{l}\text { upper } \\
\text { straight }\end{array}$ & $\begin{array}{l}\text { right } \\
\text { curve }\end{array}$ & $\begin{array}{l}\text { lower } \\
\text { straight }\end{array}$ & $\begin{array}{l}\text { left } \\
\text { curve }\end{array}$ \\
\hline \hline 1 & $13.1(6.2)$ & $14.3(5.4)$ & $12.4(5.0)$ & $14.2(7.3)$ \\
$1 \mathrm{~b}$ & $14.3(5.1)$ & $13.7(6.4)$ & $16.5(7.6)$ & $11.4(3.8)$ \\
\hline 2 & $14.7(6.6)$ & $16.1(8.7)$ & $15.3(7.3)$ & $12.9(6.9)$ \\
\hline 3, time & $9.2(3.9)$ & $8.9(3.0)$ & $10.2(3.6)$ & $8.8(4.4)$ \\
3, tm & $14.2(6.8)$ & $14.0(8.1)$ & $14.9(10.3)$ & $12.3(4.6)$ \\
\hline 4 & $14.3(7.8)$ & $12.2(5.8)$ & $14.5(9.2)$ & $13.0(6.2)$
\end{tabular}

Table A.3.: DL (in ms) for each individual condition and experiment. Each cell contains the average over all participants, and standard deviation in brackets. In Experiment 3 , the time and $t m$ condition are reported separately.

\begin{tabular}{l||ll||ll} 
Exp & tm & time & straights & curves \\
\hline \hline 1 & $13.5(4.6)$ & $10.5(3.3)$ & $12.8(5.0)$ & $14.2(5.5)$ \\
$1 \mathrm{~b}$ & $13.9(4.4)$ & $10.9(4.4)$ & $15.4(5.6)$ & $12.5(3.6)$ \\
\hline 2 & $14.8(6.0)$ & $10.9(3.4)$ & $15.0(6.4)$ & $14.5(6.4)$ \\
3. time & $13.8(6.4)$ & $9.3(2.1)$ & $9.7(3.0)$ & $8.8(3.2)$ \\
3. tm & $13.8(6.4)$ & $9.3(2.8)$ & $14.5(8.2)$ & $13.1(5.9)$ \\
\hline 4 & $13.5(6.4)$ & $11.0(4.8)$ & $14.4(7.9)$ & $12.6(5.6)$
\end{tabular}

Table A.4.: PSE (in ms) for each experiment averaged for time, tm, straights and curves condition. Each cell contains the average over all participants, and standard deviation in brackets. In Experiment 3, the time and $t m$ condition are reported individually. 


\section{Bibliography}

[1] L. F. Abbott and P. Dayan. Theoretical Neuroscience. MIT press, 2001.

[2] M. Abeles. Corticonics: Neural circuits of the cerebral cortex. Cambridge University Press, 1991.

[3] M. Abeles. Synfire chains. Scholarpedia, 4(7):1441, 2009.

[4] M. Abeles and I. Gat. Detecting precise firing sequences in experimental data. $J$. Neurosci. Methods., 107:141-154, 2001.

[5] M. Ahrens and M. Sahani. Inferring elapsed time from stochastic neural processes. In J. Platt, D. Koller, Y. Singer, and S. Roweis, editors, Advances in Neural Information Processing Systems 20, pages 1-8. MIT Press, Cambridge, MA, 2008.

[6] D. Alais and D. Burr. The ventriloquist effect results from near-optimal bimodal integration. Curr. Biol., 14:257-262, 2004.

[7] L. G. Allan. The perception of time. Percept. Psychophys., 26(5):340-354, 1979.

[8] L. G. Allan. The influence of the scalar timing model on human timing research. Behav. Process., 444:101-117, 1998.

[9] L. G. Allan and A. B. Kristofferson. Psychophysical theories of duration discrimination. Percept. Psychophys., 16:26-34, 1974.

[10] L. G. Allan, A. B. Kristofferson, and E. W. Wiens. Duration discrimination of brief light flashes. Percept. Psychophys., 9:327-334, 1971.

[11] R. Barns and M. R. Jones. Expectancy, attention, and time. Cogn. Psychol., 41:254-311, 2000.

[12] J. M. Beggs, J. R. Moyer, J. P. McGann, and T. H. Brown. Prolonged synaptic integration in perirhinal cortical neurons. J. Neurophysiol., 83:3294-3298, 2000.

[13] G.-Q. Bi and M.-M. Poo. Synaptic modifications in cultured hippocampal neurons: Dependence on spike timing, synaptic strength, and postsynaptic cell type. J. Neurosci., 18(24):10464-10472, 1998.

[14] E. Bienenstock. A model of neocortex. Network-Comp. Neural., 6:179-224, 1995.

[15] L. A. Bizo, J. Y. Chua, F. Sanabria, and P. R. Killeen. The failure of Weber's law in time perception and production. Behav. Process., 71:201-210, 2006. 
[16] S. Blaschke. Zeitwahrnehmung in isochronen Sequenzen: Ein Vergleich verschiedener Modelle zum Einfluss des Kontextes auf die Wahrnehmungsleistung. PhD thesis, Universität Göttingen, 2009.

[17] S. Blaschke, J. Haß, J. M. Herrmann, and T. H. Rammsayer. Detecting a temporally deviating interval in an isochronous sequence with filled intervals: A test for pacemakercounter and oscillator based models. submitted, 2011.

[18] R. A. Block and D. Zakay. Prospective and retrospective duration judgments: A metaanalytic review. Psychon. Bull. Rev., 4:184-197, 1997.

[19] J. F. Brown. On time perception in visual movement fields. Psychol. Forschg., 14:233$248,1931$.

[20] S. W. Brown. Time, change, and motion: The effects of stimulus movement on temporal perception. Percept. Psychophys., 57(1):105-116, 1995.

[21] S. W. Brown. Attentional resources in timing: Interference effects in concurrent temporal and nontemporal working memory tasks. Percept. Psychophys., 59(7):1118-1140, 1997.

[22] S. W. Brown. Time and attention: Review of the literature. In Psychology of Time, pages 111-138. Emerald Group Pub Ltd., Bingley, UK, 2008.

[23] S. W. Brown and E. D. Bennett. The role of practice and automaticity in temporal and nontemporal dual-task performance. Psychol. Res., 66:80-89, 2002.

[24] C. V. Buhusi and W. H. Meck. What makes us tick? Functional and neural mechanisms of interval timing. Nature Rev. Neurosci., 6:755-765, 2005.

[25] D. V. Buonomano. Decoding temporal information: A model based on short-term synaptic plasticity. J. Neurosci., 20(3):1129-1141, 2000.

[26] D. V. Buonomano. Timing of neural responses in cortical organotypic slices. Proc. Natl. Acad. Sci., 100(8):4897-4902, 2003.

[27] D. V. Buonomano. A learning rule for the emergence of stable dynamics and timing in recurrent networks. J. Neurophysiol., 94:2275-2283, 2005.

[28] D. V. Buonomano and U. R. Karmarkar. How do we tell time? Neuroscientist, 8(1):42$51,2002$.

[29] K.-M. Chen and S.-L. Yeh. Asymmetric cross-modal effects in time perception. Acta Psychol., 130(3):225-234, 2009.

[30] A. Chiba, K.-I. Oshio, and M. Inase. Striatal neurons encoded temporal information in duration discrimination task. Exp. Brain Res., 186(4):671-676, 2008.

[31] J. Cohen. Statistical power analysis for the behavioral sciences (2nd edition). Academic Press, New York, 1988. 
[32] P. Coveney and R. Highfield. The Arrow of Time. Allen, New York, 1990.

[33] C. D. Creelman. Human discrimination of auditory duration. J. Acoust. Soc. Am., 34:582-593, 1962.

[34] S. Deneve and A. Pouget. Bayesian multisensory integration and cross-modal spatial links. J. Physiol. (Paris), 98(1-3):249-258, 2004.

[35] J. Diedrichsen, S. E. Criscimagna-Hemminger, and R. Shadmehr. Dissociating timing and coordination as functions of the cerebellum. J. Neurosci., 27(23):6291-6301, 2003.

[36] M. Diesmann. Conditions for Stable Propagation of Synchronous Spiking in Cortical Neural Networks. PhD thesis, Ruhr-Universität Bochum, 2002.

[37] M. Diesmann, M.-O. Gewaltig, and A. Aertsen. Stable propagation of synchronous spiking in cortical neural networks. Nature, 402:529-533, 1999.

[38] P. L. Divenyi and W. F. Danner. Discrimination of time intervals marked by brief acoustic pulses of various intensities and spectra. Percept. Psychophys., 21:125-142, 1977.

[39] C. Drake and M.-C. Botte. Tempo sensitivity in auditory sequences: Evidence for a multiple-look model. Percept. Psychophys., 54(3):277-286, 1993.

[40] D. Durstewitz. Self-organizing neural integrator predicts interval times through climbing activity. J. Neurosci., 23(12):5342-5353, 2003.

[41] D. Durstewitz. Neural representation of interval time. Neuroreport, 15:745-749, 2004.

[42] D. Eagleman. Human time perception and its illusions. Curr. Opin. Neurobiol., 18(2):131-136, 2008.

[43] H. Eisler. Experiments on subjective duration 1868-1975: A collection of power function exponents. Psychol. Bull., 83(6):1154-1171, 1976.

[44] B. Ermentrout. Ermentrout-Kopell canonical model. Scholarpedia, 3(3):1398, 2008.

[45] M. O. Ernst and M. S. Banks. Humans integrate visual and haptic information in a statistically optimal fashion. Nature, 415:429-433, 2002.

[46] G. T. Fechner. Elemente der Psychophysik. Breitkopf und Härtel, Leipzig, 1860.

[47] G. T. Fechner. Über die Frage des Weber'schen Gesetzes und Periodicitaetsgesetzes im Gebiete des Zeitsinnes. Hirzel, 1887.

[48] P. Fraisse. Psychology of Time. Harper and Row, New York, 1963.

[49] P. Fraisse. Time and rhythm perception. In E. C. Carterette and M. P. Friedman, editors, Handbook of Perception VIII: Perceptual Coding, New York, 1978. Academic Press. 
[50] P. Fraisse. Perception and estimation of time. Ann. Rev. Psychol., 35, 1984.

[51] E. A. Franz, H. N. Zelaznik, and A. Smith. Evidence of common timing processes in the control of manual, orofacial and speech movements. J. Mot. Behav., 24:281-287, 1992.

[52] A. Genovesio, S. Tsujimoto, and S. P. Wise. Neuronal activity related to elapsed time in prefrontal cortex. J. Neurophysiol., 95:3281-3285, 2006.

[53] G. A. Gescheider. Psychophysics: The Fundamentals. Erlbaum, 1997.

[54] D. J. Getty. Counting processes in human timing. Percept. Psychophys., 20:191-197, 1976.

[55] J. Gibbon. Scalar expenctancy theory and Weber's law in animal timing. Psychol. Rev., 84:279-325, 1977.

[56] J. Gibbon. Origins of scalar timing. Learn. Motiv., 22:3-38, 1991.

[57] J. Gibbon, R. M. Church, and W. H. Meck. Scalar timing in memory. Ann. N. Y. Acad. Sci., 423:52-77, 1984.

[58] J. Gibbon, C. Malapani, C. L. Dale, and C. R. Gallistel. Toward a neurobiology of temporal cognition: advances and challenges. Curr. Opin. Neuro., 7:170-184, 1997.

[59] Goldstone and Lhamon. Studies of auditory-visual differences in human time judgment: 1. Sounds are judged longer than lights. Percept. Mot. Skills, 39:63-82, 1974.

[60] V. V. Goloubinov. Personality factors of optimal criterion control in signal detection. In Fechner Day'96, pages 269-274, Padua, 1996.

[61] S. Grondin. From physical time to the first and second moments of psychological time. Psych. Bull., 127(1):22-44, 2001.

[62] S. Grossberg and N. A. Schmajuk. Neural dynamics of adaptive timing and temporal discrimination during associative learning. Neural Networks, 2:79-102, 1989.

[63] S. Grün. Data-driven significance estimation for precise spike correlation. J. Neurophysiol., 101:1126-1140, 2009.

[64] S. E. Guttman, L. A. Gilroy, and R. Blake. Hearing what the eyes see: Auditory encoding of visual temporal sequences. Psychol. Sci., 16:228-235, 2005.

[65] R. H. R. Hahnloser, A. A. Kozhevnikov, and M. S. Fee. An ultra-sparse code underlies the generation of neural sequences in a songbird. Nature, 419:65-70, 2002.

[66] J. Haß, S. Blaschke, T. Rammsayer, and J. M. Herrmann. A neurocomputational model for optimal temporal processing. J. Comput. Neurosci., 25:449-464, 2008.

[67] J. Hass, S. Blaschke, T. Rammsayer, and J. M. Herrmann. Detection of irregularities in auditory sequences: A neural-network approach to temporal processing. In J. Mayor, N. Ruh, and K. Plunkett, editors, Proceedings of the NCPW11, pages 167-178. World Scientific, 2009. 
[68] G. Hayon, M. Abeles, and D. Lehmann. A model for representing the dynamics of a system of synfire chains. J. Comput. Neurosci., 18:41-53, 2005.

[69] D. Heeger. Signal detection theory. http://www.cns.nyu.edu/ david/handouts/sdtadvanced.pdf, 1997.

[70] J. M. Herrmann, J. A. Hertz, and A. Prügel-Bennet. Analysis of synfire chains. NetworkComp. Neural., 6:403-414, 1995.

[71] J. A. Hertz and A. Prügel-Bennett. Learning short synfire chains by self-organization. Network-Comp. Neural., 7(2):357-363, 1996.

[72] A. V. M. Herz, T. Gollisch, C. K. Machens, and D. Jaeger. Modeling single-neuron dynamics and computations: A balance of detail and abstraction. Science, 314:80-85, 2009 .

[73] I. Hodinott-Hill, K. V. Thilo, A. Cowey, and V. Walsh. Auditory chronostasis: Hanging on the telephone. Curr. Biol., 12:1779-1781, 2008.

[74] A. Höring. Versuche über das Unterscheidungsvermögen des Hörsinns für Zeitgrößen. Laupp, Tübingen, Germany, 1864.

[75] A. R. Hunt, C. S. Chapman, and A. Kingstone. Taking a long look at action and time perception. J. Exp. Psychol. (Hum. Percept.), 34(1):125-136, 2008.

[76] Y. Ikegaya, G. Aaron, R. Cossart, D. Aronov, I. Lampl, D. Ferster, and R. Yuste. Synfire chains and cortical songs: Temporal modules of cortical activity. Science, 304(5670):559$564,2004$.

[77] R. B. Ivry. The representation of temporal information in perception and motor control. Curr. Opin. Neurobiol., 6:851-857, 1996.

[78] R. B. Ivry and R. E. Hazeltine. Perception and production of temporal intervals across a range of durations: Evidence for a common timing mechanism. J. Exp. Psychol. (Hum. Percept.), 21:3-18, 1995.

[79] R. B. Ivry and S. W. Keele. Timing functions of the cerebellum. J. Cogn. Neurosci., 1:136-152, 1989.

[80] R. B. Ivry and J. E. Schlerf. Dedicated and intrinsic models of time perception. Trends Cogn. Sci., 12(7):273-280, 2008.

[81] R. B. Ivry and R. M. Spencer. The neural representation of time. Curr. Opin. Neurobiol., 14:225-232, 2004.

[82] E. Izhikevich. Polychronization: Computation with spikes. Neural Comput., 18:245-282, 2006.

[83] E. M. Izhikevich. Which model to use for cortical spiking neurons. IEEE T Neural Networ, 15:1063-1070, 2004. 
[84] A. Johnston, D. H. Arnold, and S. Nishida. Spatially localized distortions of event time. Curr. Biol., 16:472-479, 2006.

[85] M. R. Jones. Time, our lost dimension: Toward a new theory of perception, attention, and memory. Psychol. Rev., 83:323-355, 1976.

[86] C. Kaernbach. Simple adaptive testing with the weighted up-down method. Percept. Psychophys., 49:227-229, 1991.

[87] R. Kanai, C. L. E. Paffen, H. Hogendoorn, and F. A. J. Verstraten. Time dilation in dynamic visual display. J. Vision, 6:1421-1430, 2006.

[88] R. Kanai and M. Watanabe. Visual onset expands subjective time. Percept. Psychophys., 68:1113-1123, 2006.

[89] E. R. Kandel, J. H. Schwartz, and T. M. Jessell. Principles of Neural Science. Appleton \& Lange, 1991.

[90] U. R. Karmarkar and D. V. Buonomano. Timing in the absence of clocks: Encoding time in neural network states. Neuron, 53(3):427-438, 2007.

[91] A. Karniel and F. Mussa-Ivaldi. Sequence, time or state representation: How does the motor control system adapt to variable environments? Biol. Cybn., 89:10-21, 2003.

[92] S. M. Kay. Fundamentals of Statistical Signal Processing, Volume I: Estimation Theory. Prentice-Hall, New York, 1993.

[93] S. W. Keele, R. A. Pokorny, D. M. Corcos, and R. B. Ivry. Do perception and motor production share common timing mechanisms: A correlational analysis. Acta Psychol., 60:173-191, 1985.

[94] G. Keppel. Design and analysis: A researcher's handbook. Prentice Hall, Englewood Cliffs, NJ, 1991.

[95] P. R. Killeen and J. G. Fetterman. A behavioral theory of timing. Psychol. Rev., 95:274-295, 1988.

[96] P. R. Killeen and T. Taylor. How the propagation of error through stochastic counters affects time discrimination and other psychophysical judgments. Psychol. Rev., 107:430459, 2000 .

[97] P. R. Killeen and N. A. Weiss. Optimal timing and the weber function. Psychol. Rev., 94(4):455-468, 1987.

[98] K. Kitano, H. Okamoto, and T. Fukai. Time representing cortical activities: two models inspired by prefrontal persistent activity. Biol. Cybn., 88:387-394, 2003.

[99] S. K. E. Koekkoek, H. C. Hulscher, B. R. Dortland, R. A. Hensbroek, Y. Elgersma, T. J. H. Ruigrok, and C. I. D. Zeeuw. Cerebellar LTD and learning-dependent timing of conditioned eyelid responses. Science, 301:1736-1739, 2003. 
[100] B. Kollmeier. Audiologie, Kapitel 5: Psychophysik des normalen und gestörten Gehörs. http://medi.uni-oldenburg.de/download/docs/lehre/kollm_audiologie/ audiol5.pdf.

[101] K. P. Körding, U. Beierholm, W. J. Ma, S. Quartz, J. B. Tenenbaum, and L. Shams. Causal inference in multisensory perception. PLoS One, 2(9):e943, 2007.

[102] A. B. Kristofferson. A quantal step function in duration discrimination. Percept. Psychophys., 27:300-306, 1980.

[103] P. N. Kugler and M. T. Turvey. Information, natural law, and the self-assembly of rhythmic movement. Erlbaum, Hillsdale, NJ, 1987.

[104] E. Lapid, R. Ulrich, and T. H. Rammsayer. On estimating the difference limen in duration discrimination tasks: A comparison of the 2AFC and the reminder task. Percept. Psychophys., 70(2):291-305, 2008.

[105] M. A. Lebedev, J. E. O'Doherty, and M. A. L. Nicolelis. Decoding of temporal intervals from cortical ensemble activity. J. Neurophysiol., 99:166-186, 2008.

[106] M. R. Leek. Adaptive procedures in psychophysical research. Percept. Psychophys., 63(8):1279-1292, 2001.

[107] H. Lejeune and J. H. Wearden. The comparative psychology of fixed-interval performance: Some quantitative analyses. Learn. Motiv., 22:84-111, 1991.

[108] H. Lejeune and J. H. Wearden. Scalar properties in animal timing: Conformity and violations. Q. J. Exp. Psychol., 59(11):1875-1908, 2006.

[109] M. I. Leon and M. N. Shadlen. Representation of time by neurons in the posterior parietal cortex of the macaque. Neuron, 38:317-327, 2003.

[110] P. A. Lewis and R. C. Miall. Distinct systems for automatic and cognitively controlled time measurement: evidence from neuroimaging. Curr. Opin. Neurobiol., 13:250-255, 2003.

[111] W. Maass, T. Natschläger, and H. Markram. Real-time computing without stable states: A new framework for neural computation based on perturbations. Neural Comput., 14:2531-2560, 2002.

[112] F. Macar, H. Lejeune, M. Bonnet, A. Ferrara, V. Pouthas, F. Vidal, and P. Maquet. Activation of the supplementary motor area and of attentional networks during temporal processing. Exp. Brain Res., 142:475-485, 2002.

[113] F. Macar and F. Vidal. Event-related potentials as indices of time processing: A review. J. Psychophysiol., 18:89-104, 2004.

[114] F. Macar, F. Vidal, and L. Casini. The supplementary motor area in motor and sensory timing: evidence from slow brain potential changes. Exp. Brain Res., 125(3):271-280, 1999. 
[115] E. Mach. Untersuchungen über den Zeitsinn des Ohres. Sitzungsberichte der mathematisch-naturwissenschaftlichen Classe der Kaiserlichen Akademie der Wissenschaften, 51:133-150, 1887.

[116] A. Machado. Learning the temporal dynamics of behavior. Psychol. Rev., 104:241-265, 1997.

[117] C. K. Machens, R. Romo, and C. D. Brody. Flexible control of mutual inhibition: A neural model of two-interval discrimination. Science, 307(5712):1121-1124, 2005.

[118] K. Mainzer. The Little Book of Time. Springer, New York, 2002.

[119] M. S. Matell and W. H. Meck. Cortico-striatal circuits and interval timing: coincidence detection of oscillatory processes. Cogn. Brain Res., 21:139-170, 2004.

[120] M. S. Matell, W. H. Meck, and M. A. L. Nicolelis. Interval timing and the encoding of signal duration by ensembles of cortical and striatal neurons. Behav. Neurosci., 117(4):760-773, 2003.

[121] M. M. Mauk and D. V. Buonomano. The neural basis of temporal processing. Annu. Rev. Neurosci, 27:307-340, 2004.

[122] J. D. McAuley. Perception of Time as Phase: Toward and Adaptive-Oscillator Model of Rhythmic Pattern Processing. PhD thesis, Indiana University, 1995.

[123] J. D. McAuley and M. R. Jones. Modeling effects of rhythmic context on perceived duration: A comparison of interval and entrainment approaches to short-interval timing. J. Exp. Psychol. (Hum. Percept.), 29:1102-1125, 2003.

[124] D. McLelland and O. Paulsen. Cortical songs revisited: A lesson in statistics. Neuron, 53(3):413-425, 2007.

[125] J. M. E. McTaggart. The unreality of time. Mind, 17:457-474, 1908.

[126] W. H. Meck. Attentional bias between modalities: Effect on the internal clock, memory, and decision stages used in animal time discrimination. Ann. N. Y. Acad. Sci., 423(528541), 1984.

[127] W. H. Meck. Affinity for the dopamine D2 receptor predicts neuroleptic potency in decreasing the speed of an internal clock. Pharmacol. Biochem. Behav., 25:1185-1189, 1986.

[128] W. H. Meck. Neuropharmacology of timing and time perception. Cogn. Brain Res., 3:227-242, 1996.

[129] W. H. Meck. Neuroanatomical localization of an internal clock: a functional link between mesolimbic, nigrostriatal, and mesocortical dopaminergic systems. Brain Res., 1109:93107, 2006.

[130] W. H. Meck, T. B. Penney, and V. Pouthas. Cortico-striatal representation of time in animals and humans. Curr. Opin. Neurobiol., 18:145-152, 2008. 
[131] J. F. Medina, K. S. Garcia, W. L. Nores, N. M. Taylor, and M. D. Mauk. Timing mechanisms in the cerebellum: Testing predictions of a large-scale computer simulation. J. Neurosci., 20(14):5516-5525, 2000.

[132] D. V. Meegan, R. N. Aslin, and R. A. Jacobs. Motor timing learned without motor training. Nature Neurosci., 3:860-862, 2000.

[133] C. Miall. The storage of time intervals using oscillating neurons. Neural Comput., 3(1):359-371, 1989.

[134] A. Mita, H. Mushiake, K. Shima, Y. Matsuzaka, and J. Tanji. Interval time coding by neurons in the presupplementary and supplementary motor areas. Nature Neurosci., 12(4):502-507, 2009.

[135] M. C. Morrone, J. Ross, and D. Burr. Saccadic eye movements cause compression of time as well as space. Nature Neurosci., 8:950-954, 2005.

[136] F. Moss, L. M. Ward, and W. G. Sannita. Stochastic resonance and sensory information processing: a tutorial and review of application. Clin. Neurophysiol., 115(2):267-281, 2003.

[137] J. J. New and B. J. Scholl. Subjective time dilation: Spatially local, object-based, or a global visual experience? J. Vision, 9(2):4, 1-11, 2009.

[138] H. Niki and M. Watanabe. Prefrontal and cingulate unit activity during timing behavior in the monkey. Brain Res., 171(2):213-224, 1979.

[139] R. A. Normann and F. S. Werblin. Control of retinal sensitity I: Light and dark adaptation of vertebrate rods and cones. J. Gen. Physiol., 63:37-61, 1974.

[140] H. Okamoto and T. Fukai. Neural mechanism for a cognitive timer. Phys. Rev. Lett., 86(17):3919-3922, 2001.

[141] H. Pashler. Perception and production of brief durations: Beat-based versus intervalbased timing. J. Exp. Psychol. (Hum. Percept.), 27:485-493, 2001.

[142] T. B. Penney, J. Gibbon, and W. H. Meck. Differential effects of auditory and visual signals on clock speed and temporal memory. J. Exp. Psychol. (Hum. Percept.), 26(6):1770-1787, 2000.

[143] S. P. Perrett, B. P. Ruiz, and M. D. Mauk. Cerebellar cortex lesions disrupt learningdependent timing of conditioned eyelid responses. J. Neurosci., 13:1708-1718, 1993.

[144] E. Pöppel. Eine neurophysiologische Definition des Zustands "bewußt". In E. Pöppel, editor, Gehirn und Bewusstsein, pages 17-32. VCH, Weinheim, 1989.

[145] K. Popper. Logik der Forschung. Springer, Wien, 1935.

[146] W. D. Poynter. Judging the duration of time intervals: A process of remembering segments of experience. In I. Levin and D. Zakay, editors, Time and human cognition: A life-span perspective, Amsterdam, 1989. Elsevier. 
[147] M. B. Priestley. Spectral analysis and time series. Academic Press, London, New York, 1982.

[148] T. H. Rammsayer. Neuropharmacological evidence for different timing mechanisms in humans. Q. J. Exp. Psychol., 52(B):273-286, 1999.

[149] T. H. Rammsayer and S. Grondin. Psychophysics of human timing. In R. Miller, editor, Time and the brain, pages 157-167. Harwood Academic, 2000.

[150] T. H. Rammsayer and S. D. Lima. Duration discrimination of filled and empty auditory intervals: Cognitive and perceptual factors. Percept. Psychophys., 50:565-574, 1991.

[151] T. H. Rammsayer and R. Ulrich. No evidence for qualitative differences in the processing of short and long temporal intervals. Acta Psychol., 120:141-171, 2005.

[152] C. Rao. Linear statistical inference and its applications. Prentice-Hall, New York, 1973.

[153] S. M. Rao, D. L. Harrington, K. Y. Haaland, J. A. Bobholz, R. W. Cox, and J. R. Binder. Distributed neural systems underlying the timing of movements. J. Neurosci., 17(14):5528-5535, 1997.

[154] S. M. Rao, A. R. Mayer, and D. L. Harrington. The evolution of brain activation during temporal processing. Nature Neurosci., 4:317-323, 2001.

[155] S. D. Robertson, H. N. Zelaznik, D. A. Lantero, K. G. Bojczyk, R. M. Spencer, J. G. Doffin, and T. Schneidt. Correlations for timing consistency among tapping and drawing tasks: Evidence against a single timing process for motor control. J. Exp. Psychol. (Hum. Percept.), 25(5):1316-1330, 1999.

[156] C. O. Roelofs and W. P. C. Zeeman. Influence of different sequences of optical stimuli on the estimation of duration of a given interval of time. Acta Psychol., 8:89-128, 1951.

[157] D. Rose and J. Summers. Duration illusions in a train of visual stimuli. Perception, 24:1177-1187, 1995.

[158] S. Roux, M. Coulmance, and A. Riehle. Context-related representation of timing processes in monkey motor cortex. Eur. J. Neurosci., 18:1011-1016, 2003.

[159] T. J. Shors and L. D. Matzel. Long-term potentiation: What's learning got to do with it? Behav. Brain Sci., 20:597-655, 1997.

[160] G. Silberberg, M. Bethge, H. Markram, K. Pawelzik, and M. Tsodyks. Dynamics of population rate codes in ensembles of neocortical neurons. J. Neurophysiol., 91:704$709,2004$.

[161] P. Simen and F. Balci. Adaptive interval timing by a noisy integrate-and-fire model. in preparation, 2009.

[162] J. E. R. Staddon and J. J. Higa. Time and memory: Towards a pacemaker-free theory of interval timing. J. Exp. Anal. Behav., 71(2):215-251, 1999. 
[163] S. S. Stevens. Psychophysics: Introduction into its perceptual, neural and social prospects. Wiley, New York, 1975.

[164] G. ten Hoopen, R. Hartsuiker, T. Sasaki, Y. Nakajima, M. Tanaka, and T. Tsumura. Auditory isochrony: Time-shrinking and temporal patterns. Perception, 24:577-593, 1995.

[165] P. Thier, P. W. Dicke, R. Haas, and S. Barash. Encoding of movement time by populations of cerebellar purkinje cells. Nature, 405:72-76, 2000.

[166] E. A. C. Thomas and N. E. Cantor. Interdependence between the processing of temporal and non-temporal information. In J. Requin, editor, Attention and Performance, Hillsdale, NJ, 1978. Erlbaum.

[167] E. A. C. Thomas and W. B. Weaver. Cognitive processing in time perception. Percept. Psychophys., 17:363-367, 1975.

[168] K. H. Tieu, A. L. Keidel, J. P. McGann, B. Faulkner, and T. H. Brown. Perirhinalamygdala circuit-level computational model of temporal encoding in fear conditioning. Psychobiology, 27(1):1-25, 1999.

[169] M. Treisman. Temporal discrimination and the indifference interval: Implications for a model of the "internal clock". Psychol. Monogr., 77(13):1-31, 1963.

[170] M. Treisman, A. Faulkner, P. L. N. Naish, and D. Brogan. The internal clock: Evidence for a temporal oscillation underlying time perception with some estimates of its characteristic frequency. Perception, 19:705-743, 1990.

[171] P. U. Tse, J. Intriligator, J. Rivest, and P. Cavanagh. Attention and the subjective expansion of time. Percept. Psychophys., 66(7):1171-1189, 2004.

[172] G. G. Turrigiano. Homeostatic plasticity in neuronal networks: the more things change, the more they stay the same. Trends Neurosci., 22:221-227, 1999.

[173] Ulrich, Nitschke, and Rammsayer. Perceived duration of expected and unexpected stimuli. Psychol. Res., 70(2):77-87, 2006.

[174] R. Ulrich, J. Nitschke, and T. H. Rammsayer. Crossmodal temporal discrimination: Assessing the predictions of a general pacemaker-counter model. Percept. Psychophys., 68(7):1140-1152, 2006.

[175] M. C. W. van Rossum, G.-Q. Bi, and G. G. Turrigiano. Stable hebbian learning from spike timing-dependent plasticity. J. Neurosci., 20(23):8812-8821, 2000.

[176] V. van Wassenhove, D. V. Buonomano, S. Shimojo, and L. Shams. Distortions of subjective time perception within and across senses. PLoS ONE, 3(1):e1437, 2008.

[177] K. Vierordt. Der Zeitsinn nach Versuchen. Laupp, Tübingen, Germany, 1868.

[178] P. Viviani, P. Campadelli, and P. Mounoud. Visuo-manual pursuit tracking of human two-dimensional movements. J. Exp. Psychol. (Hum. Percept.), 13:62-78, 1987. 
[179] P. Viviani and T. Flash. Minimum-jerk, two-thirds power law, and isochrony: Converging approaches to movement planning. J. Exp. Psychol. (Hum. Percept.), 21(1):32-53, 1995.

[180] P. Viviani and P. Mounoud. Perceptuo-motor compatibility in pursuit tracking of twodimensional movements. J. Motor. Behav., 22:407-443, 1990.

[181] J. T. Walker and K. J. Scott. Auditory-visual conflicts in the perceived duration of lights, tones, and gaps. J. Exp. Psychol. (Hum. Percept.), 7:1327-1339, 1981.

[182] X.-J. Wang. Synaptic reverberation underlying mnemonic persistent activity. Trends in Neurosciences, 24(8):455-463, 2001.

[183] J. H. Wearden. The power law and weber's law in fixed-interval postreinforcement pausing: A scalar timing model. Q. J. Exp. Psychol., 37B:191-212, 1985.

[184] J. H. Wearden. Temporal bisection in humans with longer stimulus durations. Q. J. Exp. Psychol., 50B:79-94, 1997.

[185] J. H. Wearden, H. Edwards, M. Fakhri, and A. Percival. Why "sounds are judged longer than lights": Application of a model of the internal clock in humans. Q. J. Exp. Psychol., 51B:97-120, 1998.

[186] J. H. Wearden and H. Lejeune. Scalar properties in human timing: Conformity and violations. Q. J. Exp. Psychol., 61(4):569-587, 2008.

[187] T. Wennekers and G. Palm. Controlling the speed of synfire chains. In ICANN, pages 451-456, 1996.

[188] T. Yamazaki and S. Tanaka. Neural modeling of an internal clock. Neural Comput., 17:1032-1058, 2006.

[189] H. N. Zelaznik, R. M. Spencer, and R. B. Ivry. Dissociation of explicit and implicit timing in repetitive tapping and drawing movements. J. Exp. Psychol. (Hum. Percept.), 28:575-588, 2002.

[190] H. N. Zelaznik, R. M. C. Spencer, and R. B. Ivry. Behavioral analysis of human movement timing. In Psychology of Time, pages 233-260. Emerald Group Pub Ltd., Bingley, UK, 2008. 


\section{Acknowledgments}

There are many people who supported me during the work on this thesis, to which I owe many thanks. In this final part of the work, I will try to relieve this dept just a little bit by thanking them for their help.

First of all, I would like to thank Theo Geisel for letting me join his truly unique research group already at the stage of an undergraduate, and for his constant trust and support. After all these years, is hard for me to imagine a better environment for both scientific and personal growth than Haus 2, Bunsenstrasse 10, Göttingen. I am proud that you allowed me to be a part of it.

Michael Herrmann has been an excellent supervisor throughout my scientific life. He always maintained the delicate balance between supervision and support on the one hand, but also giving me enough freedom and encouragement to find my own ways in science. I often profited from his broad knowledge and his infamous tendency to think in unfamiliar directions, whether scientific or philosophical. And I learned a lot more from him about statistics and information theory then I thought I would want to - at the beginning. Thank you for everything.

I also profited a lot from the intense discussions with my collaborator Stefan Blaschke. I learned from him to really take the psychological view on how the brain works, and a myriad of technical details about psychological data analysis and experimental design. Without him, I would probably still stare at an analysis of variance calculation as if it was a piece of witchcraft. Thank you for guiding me to these new perspectives.

To that end, I also want to thank my collaborator Thomas Rammsayer, for sharing with me his experience in psychophysical time perception research. I also profited a lot from the helpful comments of the members of my thesis committee, Stefan Treue and Kristian Folta. Thank you for your time in the committee, and for pointing out weak and strong points of this work which I would probably not have seen myself.

When writing a doctoral thesis, one often feels like doing a $\mathrm{PhD}$ in science administration and computer science at the same time. But in my case, there were people who kept providing priceless support, so I could concentrate on getting one degree at a time. My thanks go to Regina Wunderlich, Katharina Jeremias, Tanja Gindele and Corinna Trautsch for their help with all the - only seemingly - small perils and pitfalls of daily office life, Yorck-Fabian Beensen, Denny Fliegner and Barbara Guichemer for unbelievably smooth computer support, and to Tobias Niemann and Kirsten Pölker and her team at the GGNB office for answering all my countless questions with remarkable patience.

When it comes to patience and answering questions, I must of course not forget Frank Hesse, who had the sorry fate of always being a few steps ahead of me in becoming a GGNB 
student and graduating from it, and thus being the target of all the questions that could only be answered by a person concerned. Thank you, Frank.

Frank Hesse and Barbara Guichemer also had their own way to contribute to the special atmosphere in the institute - they both were my office mates for some period of time. It is always much nicer to go to work when there is good company around. You truely did this job very well.

In fact, these kinds of thanks can easily be extend to the entire research group. Although the group is constantly growing and changing these days, so you can never be sure which names you would actually have to include in the list, one thing has not changed since the time of my diploma thesis - you are still a great bunch of people!

I would also like to gratefully acknowledge the contributions of all those people which stimulated my scientific mind with fruitful discussions. Among these people are Moshe Abeles, Tamar Flash, Markus Diesmann and Mina Teicher. These people from the DIP project "compositionality" as well as Mona Bornschlegl and Armin Biess provided me with recurring feedback on my work, especially about the synfire chain and motor control part of my thesis, which I deem invaluable. Discussions with Misha Ahrens and Maneesh Sahani greatly stimulated the research on the information theory of time. I am also grateful for the stimulating discussions I had with Timo von Oertzen, Dean Buonomano, Patrick Simen and Stefan Glasauer. Thank you all for opening my mind for new perspectives.

Special thanks go to those persons who made the experimental part of this thesis possible. First of all, Svenja Fikurat did a great job in recruiting and testing almost one hundred participants in the time and motion experiments. Thank you very much for your steady and reliable work. Also, I recruited several participants myself, whenever we ran short of undergraduate psychology students. I usually promised them my eternal thanks and a place in the acknowledgments of my thesis. Well, Tobias, Katharina, Vincent, Christiane, Martin, Georg, Pinar, Demian, Olav, Janina, Christine, Annette, Doriet, Sascha, Ayse, Marie and Caro... here you go: Thank you!

Last but not least, I want to thank those people who were not directly involved in the scientific aspects of my life, but without whom this thesis would have nevertheless been impossible. I thank my parents Jutta and Jochen for everything they gave me, including the trust and support during difficult times, whatever the nature. And finally, I sincerely thank my beloved partner, Christiane. She gave me more power and will to keep going then she might realize. And she never gets tired to show me that life doesn't end at the office door. In neither direction. Thank you.

I acknowledge financial support during this thesis by a grant from the Bundesministerium für Bildung und Forschung in the framework of the Bernstein Center for Computational Neuroscience Göttingen, grant number 01GQ0432. 